\title{
DOE/CE/40895--T2
}

\section{MAGNETIC LIQUEFIER FOR HYDROGEN}

\author{
Astronautics Corporation of America \\ Astronautics Technology Center \\ 5800 Cottage Grove Road \\ Madison, WI 53716
}

Revised as of July 2, 1992

Contract No. DE-AC02-90CE40895

Sponsored by:

U.S. Department of Energy

Office of Industrial Technologies

1000 Independence Street

Washington, D.C.

Technical Direction/Monitoring:

Chicago Operations Office

Argonne National Laboratory

Argonne, IL 


\section{Table of Contents}

Executive Summary.................................................................................

1 Introduction ..................................................................................

1.1 System Description ........................................................................... 2

$1.2 \quad$ Magnetic Refrigeration ........................................................................

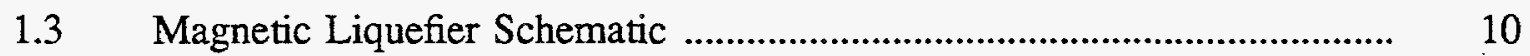

$1.4 \quad$ Liquefier System Configuration .......................................................... 12

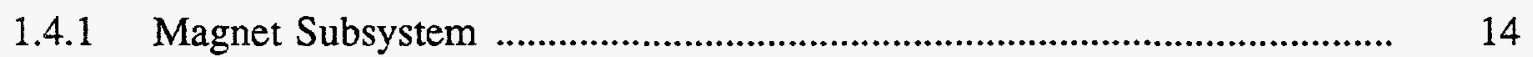

1.4.2 AMR Regenerator Bed Design ........................................................... 14

1.4.3 Support Structure and Drive Design ....................................................... 14

1.4.4 Vacuum System and Fluid System ........................................................... 14

1.4.5 Computer Control System ……......................................................... 15

$2 \quad$ Alternative Magnetic Refrigerator Concepts ………................................ 15

$2.1 \quad$ External Regenerator Magnetic Refrigerator ............................................ 16

2.2 Internal Regenerator Magnetic Refrigerator ............................................. 21

2.3 Recuperative Magnetic Refrigerator ….................................................. 23

3 The Active Magnetic Regenerative Refrigerator (AMR) Model .............. 27

$4 \quad$ Subscale Prototype Preliminary Design ................................................... 38

4.1 Proof-of-Principle Active Magnetic Regenerative Refrigerator ................ $\quad 40$

4.1.1 AMR Principle ........................................................................... 40

4.1.2 Experimental Apparatus ................................................................ 40

4.1.3 Testing Procedure ............................................................................. 44

4.2 Test Results .................................................................................. 49

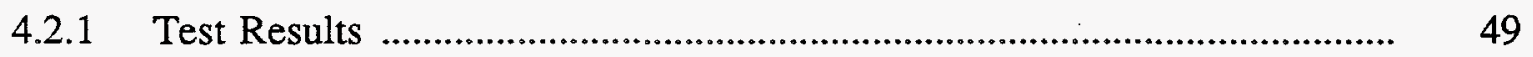

4.2.1.1 $\quad \mathrm{Er}_{0.86} \mathrm{Gd}_{0.14} \mathrm{Al}_{2}$ Test Results ................................................ 49

4.2.1.2 $\quad \mathrm{GdNi}_{2}$ Test Results ............................................................... 50

4.2.2 Comparisons to the Model ................................................................... 71

4.2.2.1 Interpretation of $\mathrm{Er}_{0.86} \mathrm{Gd}_{0.14} \mathrm{Al}_{2}$ Results .................................... 71

4.2.2.2 Interpretation of $\mathrm{GdNi}_{2}$ Results ................................................ 72

4.3 Testing Summary and Conclusion ........................................................ 82

4.4 Preliminary Rotary Configurations …………….................................... 82

4.5 Preliminary Reciprocating Configurations ……....................................... 93

4.6 AMR Staging and Modeling ……………….......................................... 97

4.7 Liquefier Scale-up Considerations ........................................................... 103

4.8 Preliminary Design Selection Criteria ........................................................ 106 
DISCLAMMER

Portions of this document may be illegible in electronic image products. Images are produced from the best available original document. 
4.9 Preliminary Design Review Modifications ................................................ 113

$5 \quad$ Detailed Design of a 0.1 Ton/Day Magnetic Liquefier ............................ 115

5.1 Configuration Component Integration ................................................. 115

$5.2 \quad$ Working Magnetic Bed Design .............................................................. 119

5.3 Reciprocating AMR Magnet Design ……............................................ 130

5.3.1 Magnet Design Specifications _.......................................................... 132

$5.4 \quad$ Auxiliary Equipment …...................................................................... 132

$5.5 \quad$ Drive System ............................................................................. 135

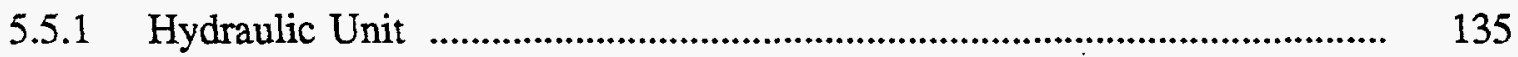

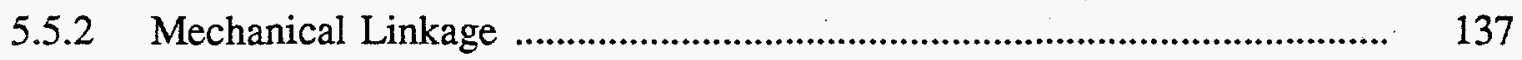

$5.6 \quad$ Vacuum System ......................................................................... 143

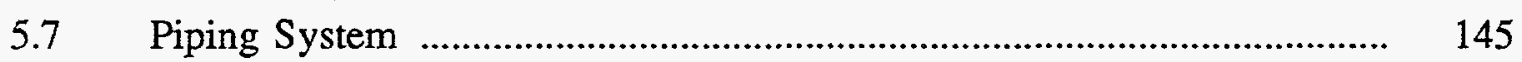

$5.8 \quad$ Prototype Instrumentation and Control .................................................. 151

$5.9 \quad$ Sensor Type and Locations ………....................................................... 151

5.10 Control Logic and Operational Flexibility ................................................ 153

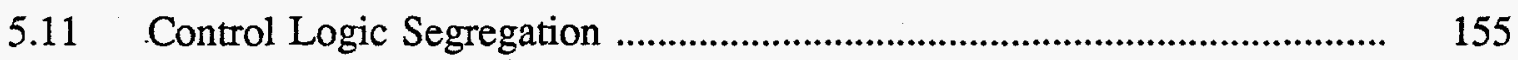

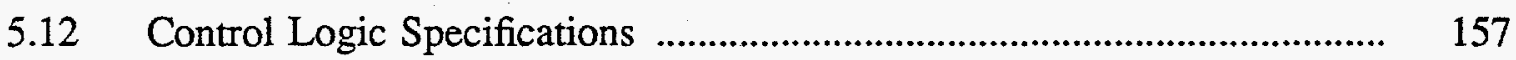

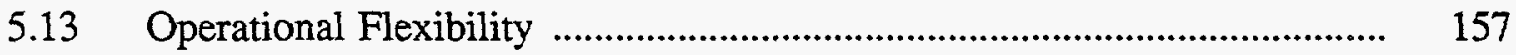

5.13.1 Anticipated Operating Parameters ......................................................... 157

$6 \quad$ Selection of Magnetic Refrigerants for the Prototype Liquefier ............... 158

$6.1 \quad$ Choice of Materials ............................................................................. 158

6.2 Magnetization Measurement Instrumentation ........................................... 161

6.2.1 Magnetization Measurements ........................................................... 162

6.3 Magnetocaloric Effect Measurement Techniques ..................................... 167

6.3.1 Magnetocaloric Effect Measurement Results ......................................... 167

6.3.2 Analysis of the Magnetocaloric Data ..................................................... 178

6.4 Applicability of the Materials to Hydrogen Liquefaction .......................... 191

$7 \quad$ Regenerator Bed Materials Fabrication ................................................. 194

7.1 Applicable Fabrication Techniques ……………....................................... 199

\section{DISCLAIMER}

This report was prepared as an account of work sponsored by an agency of the United States Government. Neither the United States Government nor any agency thereof, nor any of their employees, makes any warranty, express or implied, or assumes any legal liability or responsibility for the accuracy, completeness, or usefulness of any information, apparatus, product, or process disclosed, or represents that its use would not infringe privately owned rights. Reference herein to any specific commercial product, process, or service by trade name, trademark, manufacturer, or otherwise does not necessarily constitute or imply its endorsement, recommendation, or favoring by the United States Government or any agency thereof. The views and opinions of authors expressed herein do not necessarily state or reflect those of the United States Government or any agency thereof. 


\section{List of Figures}

Figure 1. Schematic of Magnetic Liquefier for Hydrogen

Figure 2. T-S Diagram of Ideal Ferromagnetic Refrigerant for an AMR Cycle

Figure 3. Adiabatic Temperature Change of Ideal Magnetic

Material for AMR Cycle

Figure 4. Flow Schematic for Two-Stage Hydrogen Liquefier

Figure 5. Magnetic Liquefier without Vacuum Cold Box

Figure 6. Magnetic Refrigerator using External Solid Regenerator

Figure 7.

Temperature-Entropy Diagram for Brayton-Cycle MR

Figure 8.

Calculated Temperature-Entropy Diagram for GdNi

Figure 9.

Schematic of Internal Regenerative Brayton Cycle

Magnetic Refrigerator

22

Figure 10.

Rotary Brayton Cycle MR Stages

Figure 11. Schematic of the Active Magnetic Regenerator

Figure 12. Temperature Profiles for AMR Bed/Fluid in the Infinite

Bed Thermal Mass Limit

Figure 13. Bed Temperature Profiles over an AMR Cycle 36

Figure 14. Load Curves at $7 \mathrm{~T}$ for $\mathrm{GdNi}_{2} \quad 37$

Figure 15. Key Components of the AMR Experiment 42

Figure 16. Scale Drawing of an AMR Proof-of-Principle

Experiment

Figure 17. Adiabatic Temperature Change of $\mathrm{Er}_{0.86} \mathrm{Gd}_{0.14} \mathrm{Al}_{2} \mathrm{Bed}$ Material

Figure 18. HHEX and CHEX Temperature vs. Time

$\left(\mathrm{Er}_{0.86} \mathrm{Gd}_{0.14} \mathrm{Al}_{2} 3 \mathrm{~T}\right.$, Displ. Superimposed)

Figure 19. HHEX and CHEX Temperature vs. Time

$\left(\mathrm{Er}_{0.86} \mathrm{Gd}_{0.14} \mathrm{Al}_{2}, 3 \mathrm{~T}\right)$

Figure 20. HHEX and CHEX Temperature vs. Time

$\left(\mathrm{Er}_{0.86} \mathrm{Gd}_{0.14} \mathrm{Al}_{2}, 3 \mathrm{~T}\right)$

Figure 21. CHEX, Middle Bed, and In Filter Temperatures vs. Time $\left(\mathrm{Er}_{0.86} \mathrm{Gd}_{0.14} \mathrm{Al}_{2}\right)$

Figure 22. Temperature in Bed at Five Axial Locations vs. Time

$$
\left(\mathrm{Er}_{0.86} \mathrm{Gd}_{0.14} \mathrm{Al}_{2}\right)
$$

Figure 23.

HHEX and CHEX Temperature vs. Time $\left(\mathrm{GdNi}_{2}\right)$

Figure 24.

Displacer Position vs. Time

Magnet Position vs. Time

Figure 26

Flow Impedance Differential Pressure vs. Time

Figure 27.

Displacer Differential Pressure vs. Time

Figure 28.

Gas Temperature at Bed Exit vs. Time

65

Figure 29.

Displacer Exit Temperature vs. Time

66

Figure 30. Magnet Load Cell Force vs. Time 
Figure 31. Temperature Difference Along Bed vs. CHEX Input Power (Thermal Conduction Test)

Figure 32. $\mathrm{GdNi}_{2}$ Load Curves at $7 \mathrm{~T}$ Magnetic Field

Figure 33. Computed Temperature Profiles over a Complete AMR Cycle for $\mathrm{Er}_{0.86} \mathrm{Gd}_{0.14} \mathrm{Al}_{2}$

Figure 34. Computed $1 \mathrm{~T}$ Load Curves for Various Gas Flow Rates through $\mathrm{Er}_{0.86} \mathrm{Gd}_{0.14} \mathrm{Al}_{2}$ Beds

Figure 35. Computed Temperature Profiles over a Complete AMR Cycle for $\mathrm{Er}_{0.86} \mathrm{Gd}_{0.14} \mathrm{Al}_{2}$

Figure 36. Comparison between the Modeled and Experimental Data of Run 383

Figure 37. Comparison between the Modeled and Experimental Data of Run 428

Figure 38. Comparison between the Modeled and Experimental Data of Run 430

Figure 39. Comparison between the Modeled and Experimental Data of Run 431

Figure 40. Bed Particle Size Distribution of the Original Material and the Bed Material

Figure 41. Rotary Configuration Bed Ring Concept

Figure 42. Rotary Configuration Wheel Assembly with Internal Flow Manifolding

Figure 43. Rotary Dynamic Seal Interface between Housing and Wheel

Figure 44. Single Rotary Liquefier Stage with Magnets, Housing, and Wheel

Figure 45. The Three-Stage "33" Rotary Liquefier Concept

Figure 46. The Three-Stage "31" Rotary Liquefier Concept

Figure 47. The Two-Stage "21" Rotary Liquefier Concept

90

91

Figure 48. Factors for the Comparative Evaluation of 1st\&2nd Stage Reciprocating Magnets

Figure 49. AMR Trolley Structure, Preliminary Design of a Reciprocating Stage

Figure 50. Preliminary Piping Schematic of Reciprocating Configuration with Flow Rectification

Figure 51. Schematic of Two-Stage 0.1 Ton/Day Hydrogen Liquefier

Figure 52. $\mathrm{GdNi}_{2} 7 \mathrm{~T}$ Adiabatic Temperature Change Data Compared to Ideal

Figure 53. GdPd 5 T Adiabatic Temperature Change Data Compared to Ideal

Figure 54. Magnet Configuration for the Rotary Design

Figure 55.

Typical Field Profile

110

Figure 56. Final Prototype Piping Schematic

Figure 57.

Liquefier Component Integration inside Vacuum Vessel

116

Figure 58. Bed Assembly Interface to Support Structure

Figure 59. Bed Module Exploded View 
Figure 60. Bed Module Flow Header 122

Figure 61. Bed Module Port Orientation and Features 123

Figure 62. Stacked Bed Assembly and Flow Paths 124

Figure 63. Bed Subassemblies Sealed into a G-10 Support Tube $\quad \begin{aligned} & \text { Structure } \\ & \text { B }\end{aligned}$

$\begin{array}{lll}\text { Figure 64. Bolted Tubing Joint to Bed Assembly } & 127\end{array}$

Figure 65. Bed Assembly Stress Contours 129

Figure 66. System Drive Forces $\quad 131$

Figure 67. Programmable Servo-Hydraulic Schematic 136

$\begin{array}{lll}\text { Figure 68. } & \text { Drive System with Magnets } & 138\end{array}$

Figure 69. Drive System Linear Vacuum Feed-through 139

Figure 70. Drive Mechanism Thermal Analysis Model 141

Figure 71. Heat Fluxes throughout the Drive Mechanism (B) 142

Figure 72. Simplified Vacuum System Schematic 144

Figure 73. Isometric View of Piping System Assembly 146

Figure 74. Ortho-Para Conversion Increments 148

$\begin{array}{lll}\text { Figure 75. } & \mathrm{LN}_{2} \text { Heat Exchanger Piping Connections } & 149\end{array}$

Figure 76. Modified Hierarchical Control Strategy 154

Figure 77. Magnetization of a Series of $\mathrm{GdPd}_{\mathrm{x}} \mathrm{Ni}_{1-\mathrm{x}}$ Alloy 163

Figure 78. Magnetization of a $\mathrm{Gd}\left(\mathrm{Cu}_{1-\mathrm{x}} \mathrm{Ni}_{\mathrm{x}}\right)_{2}$ Alloy

(Complex Ordering Behavior)

164

Migure 79. Magnetization of $\mathrm{Er}_{0.8} \mathrm{La}_{0.2}$ for Various Applied
Magnetic Fields

Figure 80. Hysteresis Curve for a $\mathrm{Gd}\left(\mathrm{Cu}_{1-\mathrm{x}} \mathrm{Ni}_{\mathrm{x}}\right)_{2}$ Alloy 166

Figure 81. Heat Capacity, $\mathrm{C}_{\mathrm{B}}$, for GdPd, for Various Applied Fields 168

Figure 82. Adiabatic Temperature Change, $\Delta \mathrm{T}_{\mathrm{s}}$, for GdPd 169

$\begin{array}{lll}\text { Figure 83. } \quad \mathrm{C}_{\mathrm{B}} \text { for } \mathrm{GdNi} & 170\end{array}$

$\begin{array}{lll}\text { Figure 84. } & \Delta \mathrm{T}_{\mathrm{s}} \text { for } \mathrm{GdNi} & 171\end{array}$

$\begin{array}{lll}\text { Figure 85. } & \mathrm{C}_{\mathrm{B}} \text { for } \mathrm{GdNi}_{0.25} \mathrm{Pd}_{0.75} & 172\end{array}$

$\begin{array}{lll}\text { Figure 86. } & \Delta \mathrm{T}_{\mathrm{s}} \text { for } \mathrm{GdNi} & \\ 0.25 & \mathrm{Pd}_{0.75} & 173\end{array}$

$\begin{array}{lll}\text { Figure 87. } & \mathrm{C}_{\mathrm{B}} \text { for } \mathrm{GdNi}_{0.95} \mathrm{Co}_{0.05} & 174\end{array}$

Figure 88. $\Delta \mathrm{T}_{\mathrm{s}}$ for $\mathrm{GdNi}_{0.95} \mathrm{Co}_{0.05} \quad 175$

Figure 89. $\quad \mathrm{C}_{\mathrm{B}}$ for $\mathrm{GdNi}_{2} .95$

$\begin{array}{lll}\text { Figure 90. } \Delta \mathrm{T} \text { s for } \mathrm{GdNi}_{2} & 177\end{array}$

Figure 91. $\quad \mathrm{C}_{\mathrm{B}}$ for $\mathrm{Er}_{0.86} \mathrm{Gd}_{0.14} \mathrm{Al}_{2} \quad 180$

Figure 92. $\Delta \mathrm{T}$ s for $\mathrm{Er}_{0.86} \mathrm{Gd}_{0.14} \mathrm{Al}_{2}$

Figure 93. Measurements of $\mathrm{C}_{\mathrm{B}}$ on $\mathrm{Er}_{0.75} \mathrm{Dy}_{0.25} \mathrm{Ni}$

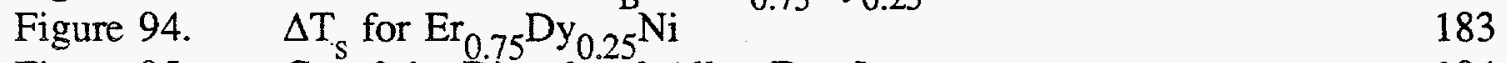

Figure 95. $\quad \mathrm{C}_{\mathrm{B}}$ of the Disordered Alloy $\mathrm{Er}_{0.8} \mathrm{La}_{0.2}$

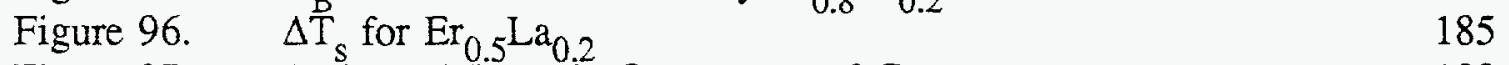

$\begin{array}{lll}\text { Figure 97. Reduced Magnetic Component of } \mathrm{C}_{\mathrm{B}} & 188\end{array}$

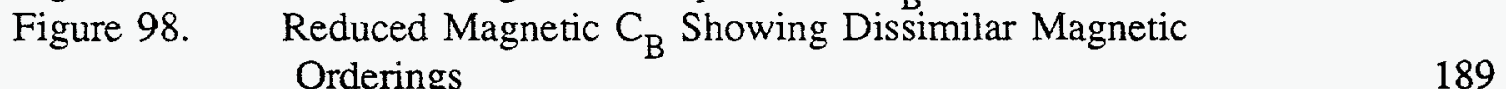

Figure 99. Comparison of $\Delta \mathrm{T}_{\mathrm{S}}$ for Several Magnetic Refrigerants $\quad 190$

Figure 100. $\Delta \mathrm{T}_{\mathrm{s}}$ for $\mathrm{GdPd}_{0.75} \mathrm{Ni}_{0.25} 192$ 
Figure 101. $\Delta \mathrm{T}_{\mathrm{s}}$ for $\mathrm{Er}_{0.8} \mathrm{La}_{0.2}$ at 7 Tesla

Figure 102. Comparison with Ideal Curve of $\Delta \mathrm{T}_{\mathrm{s}}$ at $7 \mathrm{~T}$

Figure 103. Phase Diagram for Gadolinium-Nickel System

Figure 104. Phase Diagram for Gadolinium-Palladium System

Figure 105. The ACA Particulation Apparatus

Figure 106. ACA Particulation Apparatus Schematic

202

Figure 107.

Induction Coil and Crucible of Particulation Apparatus

203

Figure 108. $\mathrm{GdNi}_{2}$ Spherical Particles Produced by Particulation Device

204

205 


\section{List of Tables}

Table 1. Alternative Concepts 15

Table 2. List of All Sensors used in the Data Acquisition

$46-48$

Table 3. Summary of $\mathrm{GdNi}_{2}$ Test Runs at $7 \mathrm{~T}$ Magnetic Field

52

Table 4. Two-Stage, 0.1 Ton/Day Liquefier Model

Table 5. Preferred Configuration Based on Selection Criteria

112

Table 6. Hydrogen Liquefier Magnet Specifications

133

Table 7. Major Components and Vendors

134

Table 8. Sensor Type and Location

Table 9. Control Loop Descriptions

156

Table 10. The Magnetic Elements

160

Table 11. Parameters Derived from Measured Heat Capacity of Magnetic Refrigerants

Appendix A Bed Assembly/Support Structure Load Interaction Summary

A-1-A-3

Appendix B

Bed Assembly Internal Parasitic Hest Leak Summary

B-1-B-6

Appendix C

Symbols and Usage

C-1-C-3 


\section{EXECUTIVE SUMMARY}

This document summarizes work done at the Astronautics Technology Center of the Astronautics Corporation of America (ACA) in Phase I of a four phase program leading to the development of a magnetic liquefier for hydrogen. This work was performed under a cost-sharing contract (DE-AC02-90CE40895) between ACA and the United States Department of Energy.

The project involves the design, fabrication, installation, and operation of a hydrogen liquefier providing significantly reduced capital and operating costs, compared to present liquefiers. To achieve this goal, magnetic refrigeration, a recently developed, highly efficient refrigeration technology, will be used for the liquefaction process. The project's four phases are:

I. Sub-Scale Prototype Design

II. Sub-Scale Prototype Fabrication and Development

III. Full Scale Liquefier Design

IV. Full Scale Fabrication and Test.

Phase I project tasks included liquefier conceptual design and analysis, preliminary design of promising configurations, design selection, and detailed design of the selected design. Fabrication drawings and vendor specifications for the selected design were completed during detailed design.

The design of a subscale, demonstration magnetic hydrogen liquefier represents a significant advance in liquefaction technology. The cost reductions that can be realized in hydrogen liquefaction in both the subscale and, more importantly, in the fullscale device are expected to have considerable impact on the use of liquid hydrogen in transportation, chemical, and electronic industries. The benefits to the nation from this technological advance will continue to have importance well into the 21 st century. 


\section{Introduction}

The Phase I report describes the complete process of selection and design. The strengths and weaknesses of the potential magnetic cycles and system configurations are detailed; several physical configurations of a refrigerator operating on the chosen cycle are described, and selection criteria are applied. The final detailed design of the subscale liquefier is given. Work ancillary to the design but essential for obtaining good performance is also presented, including a description of experimental and numerical modeling studies exploring properties of the chosen cycle, and a materials characterization program that identified the magnetic materials which are the liquefier refrigerant.

Phase II of the program is underway and initial operation of the subscale demonstration liquefier is planned for 1993. The full-scale liquefier is expected to be in commercial operation producing 1 ton/day of liquid hydrogen in 1996.

\subsection{System Description}

A system diagram of the subscale magnetic liquefier for hydrogen is shown in Figure 1. Gaseous hydrogen is precooled through heat exchange with gaseous and liquid nitrogen to approximately $77 \mathrm{~K}$. Following precooling, the hydrogen enters a counterflow heat exchanger where it is cooled to liquid at approximately $20 \mathrm{~K}$ through heat exchange with a cold helium stream produced by the magnetic refrigerator. The pressure of the emergent liquid hydrogen stream is reduced to $0.1 \mathrm{MPa}(1 \mathrm{~atm})$ by an expansion valve, and the liquid enters a storage dewar. The helium recycles through the magnetic refrigerator.

Several stages of conversion from orthohydrogen to parahydrogen are included. These O/P (Ortho-Para) converters are shown in Figure 1. Orthohydrogen and parahydrogen are two species of the hydrogen molecule. At room temperature equilibrium hydrogen is $75 \%$ orthohydrogen while at $20 \mathrm{~K}$ the equilibrium form is almost totally parahydrogen. The conversion from orthohydrogen to parahydrogen is a relatively slow exothermic process. The O/P converters catalyze the conversion process so the heat of conversion is absorbed by the cold helium stream.

The full-scale liquefier will produce 1 ton per day $(10.5 \mathrm{~g} / \mathrm{s})$ of liquid hydrogen at $20 \mathrm{~K}$ and $1 \mathrm{~atm}$. The subscale device will produce about 0.1 ton per day of liquid hydrogen $(1 \mathrm{~g} / \mathrm{s})$. The subscale design accepts, as feedstock, hydrogen gas at 4.05 $\mathrm{MPa}(40 \mathrm{~atm})$ or less, at ambient temperature (about $300 \mathrm{~K}$ ). The liquefaction efficiency is defined as the ratio of the ideal work required for hydrogen liquefaction to the actual work required. The efficiency given in Figure 1 for the magnetic liquefier is an estimate based on the work required for magnetic cycle operation and the production of liquid nitrogen used for precooling and heat exchange. The projected 


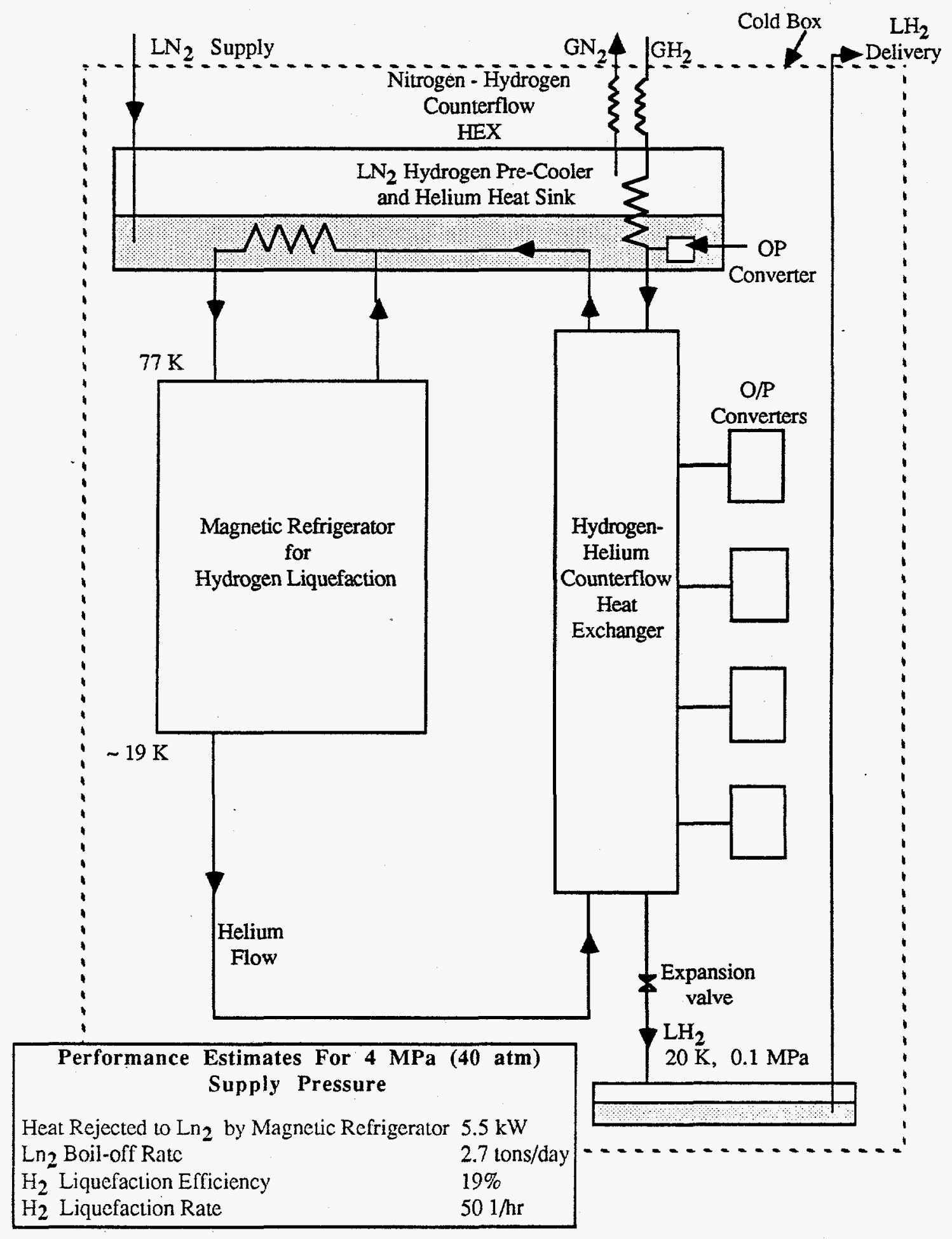

Figure 1. Schematic of Magnetic Liquefier for Hydrogen 
efficiency of the $\mathrm{LH}_{2}$ magnetic liquefier is $19 \%$, which compares favorably with the $10 \%$ to $15 \%$ efficiency of small gas-cycle liquefiers. This estimate does not include pump work within the magnetic refrigerator. Large scale commercial liquefiers, one hundred times larger than the subscale device, operate with efficiencies of about $25 \%$ of ideal. Because magnetic liquefaction technology is in an early development stage, significant efficiency increases can be expected as designs are refined and operating experience grows. Magnetic liquefier efficiencies $20 \%$ to $50 \%$ greater than conventional large liquefiers are targeted.

\subsection{Magnetic Refrigeration}

Hydrogen is liquefied in the proposed design through heat exchange with a helium stream cooled by a magnetic refrigerator. Magnetic refrigeration is an application of the magnetocaloric effect, which is the reversible heating and cooling of a ferromagnetic material upon the application and removal of a magnetic field. It is analogous to heating and cooling of a gas upon compression and expansion. In contrast to gas compression and expansion, the magnetization process is highly reversible in magnetically soft materials at frequencies and in configurations where eddy current heating is negligible. The magnetocaloric effect was discovered early in this century and refrigerators based on it have been used in research laboratories for almost 60 years. Applications such as the liquefier described herein have been possible only during the past few years, as a direct result of development work at ACA.

A magnetic refrigerator operates on a cycle driven by changes in the temperature of a magnetic working material caused by the magnetocaloric effect. The magnetic working material's adiabatic temperature change is the change in temperature exhibited by a thermally isolated element of magnetic material when the external magnetic field changes. For ferromagnetic materials near their Curie temperature, an adiabatic temperature change of $1 \mathrm{~K}$ to $2 \mathrm{~K}$ for each Tesla of magnetic field change is possible. With superconducting magnets, field changes of $5 \mathrm{~T}$ to $7 \mathrm{~T}$ are achieved easily, producing temperature changes of about $10 \mathrm{~K}$ in the magnetic material. For the subscale liquid nitrogen precooled magnetic liquefier, a temperature span of about $55 \mathrm{~K}$ is required in the magnetic stage. A regenerative or recuperative refrigeration cycle is required for operation over a temperature span larger than the magnetic material's adiabatic temperature change. For this reason, magnetic cycles appropriate for the liquefier include the Brayton, Ericsson, Stirling, and the Active Magnetic Regenerator (AMR) cycle.

The AMR cycle was chosen for the liquefier. In refrigerators operating on this cycle, a matrix consisting of the magnetic working material suffused with a fluid is alternately magnetized and demagnetized so that it warms and cools. After each field change, the fluid, typically gaseous helium, is forced to flow through the matrix, effecting heat transfer between the fluid and the matrix and, eventually, between the fluid and external heat exchangers. The matrix performs similar to the regenerator 
found in conventional regenerative refrigeration cycles such as the Stirling or Brayton cycle, with a key difference: the temperature of the magnetic matrix material changes in response to the external magnetic field and so the material acts both as a regenerator and a refrigerant.

The regenerator in a conventional gas-cycle refrigerator is a passive thermal storage medium absorbing or rejecting heat during different parts of the cycle. Often the regenerator is referred to analogously as a "thermal sponge" or "thermal flywheel," emphasizing its storage role. In the AMR, a description of the refrigerator's operating cycle must include the active role of the magnetic matrix in the refrigeration process and its passive role as a regenerator.

Consider an AMR refrigerator cycle, beginning with demagnetization of the magnetic material. During demagnetization each element of the matrix cools because of the magnetocaloric effect to the lowest temperature it will experience during the cycle. The magnetic matrix plays an active role in the refrigeration process during this part of the cycle as it is the source of heat exchange fluid cooling. The matrix temperature varies from the hot sink temperature at the high temperature end to a temperature below the cold source temperature at the cold end. Helium is then forced to flow from the hot to cold end of the matrix, rejecting heat to the matrix and cooling; it emerges from the cold end at a temperature below the cold source temperature, absorbing heat from the cold source. During this part of the cycle the matrix behaves like the regenerator in a conventional regenerative refrigerator. The matrix is then magnetized, and the temperature of the matrix and heat exchange fluid within it rises because of the magnetocaloric effect. The cold end temperature is at the cold source temperature and the hot end temperature is above the hot sink temperature. During this part of the cycle, the matrix again takes an active role in the refrigeration process. Lastly, helium is forced to flow from the cold to the hot end of the matrix. As it flows through the matrix, the helium absorbs heat from the matrix until it emerges from the hot end, at a temperature above that of the hot sink. The helium rejects heat to the sink. The cycle is complete, and thermal energy has been transported from the cold source to the hot sink by fluid convection; the AMR is a refrigerator.

The matrix material and configuration selected for AMR use is guided by the matrix's dual role of refrigerant and regenerator. Efficient refrigerator performance results from choosing materials with appropriate magnetocaloric properties. Effective regenerator performance results from several characteristics:

- good heat transfer between the matrix material and the helium heat transfer fluid,

- small pressure drops associated with the flow impedance experienced by the heat transfer fluid,

- a sufficiently larger heat capacity of the matrix material relative to the heat transfer fluid, 
- small longitudinal thermal conduction through the matrix material, and

- the ability to withstand mechanical stresses associated with magnetic field gradients and fluid flows.

Design is also determined by practical considerations such as cost and prior experience. In the liquefier, for example, potential physical configurations of the matrix included perforated plates, parallel plates, and particle beds. Particle beds of spheres were chosen because of prior experience with our laboratory AMR testing device, and the expected low cost of fabrication.

Temperature-Entropy (T-S) diagrams for magnetic materials, with magnetic field as a parameter, are a common method for presenting the thermomagnetic properties of materials. Figure 2 is a Temperature-Entropy (T-S) diagram for an ideal ferromagnetic material for AMR refrigeration, at temperatures below the Curie point. For the ideal AMR-cycle material, the T-S curves at constant magnetic field are straight lines passing through the origin; alternatively stated, the adiabatic temperature change of an ideal AMR refrigerant operating between two fixed magnetic field values is linear in temperature, vanishing at $0 \mathrm{~K}$. Also shown in the figure are typical cycles followed by different elements of magnetic material in the matrix over the AMR cycle. The cycle, followed by a material element in this idealized case, is bounded by constant field and entropy lines. This cycle is a magnetic Brayton cycle. The AMR-cycle refrigeration results from the concerted action of many Brayton cycle magnetic refrigerators acting in series.

Choosing the ideal material for liquefier use, as opposed to that for a refrigerator, requires consideration of the differences between a refrigerator and the proposed liquefier. There are two key differences:

- In a refrigerator, all the fluid that flows from the hot end of the matrix to the cold end during one part of the cycle is returned by flow from the cold end of the matrix to the hot end of the matrix during another part of the cycle. Over the cycle the flow is balanced. In the liquefier proposed herein, there is a flow imbalance, with some of the cold helium emerging from the cold end diverted to flow through the counterflow helium-hydrogen heat exchanger. Over a cycle more helium flows from the hot end to the cold end of the matrix than in the opposite direction.

- The liquefier does not require any net cooling power at its lowest temperature, in contrast to a refrigerator which is designed to produce maximum cooling at its lowest operating temperature. The cooling power of the emergent cold helium stream in the liquefier need only be sufficient to cool the hydrogen stream from $77 \mathrm{~K}$ to $20 \mathrm{~K}$ and to absorb the thermal load of the expansion valve; no additional cooling at $20 \mathrm{~K}$ is required. 


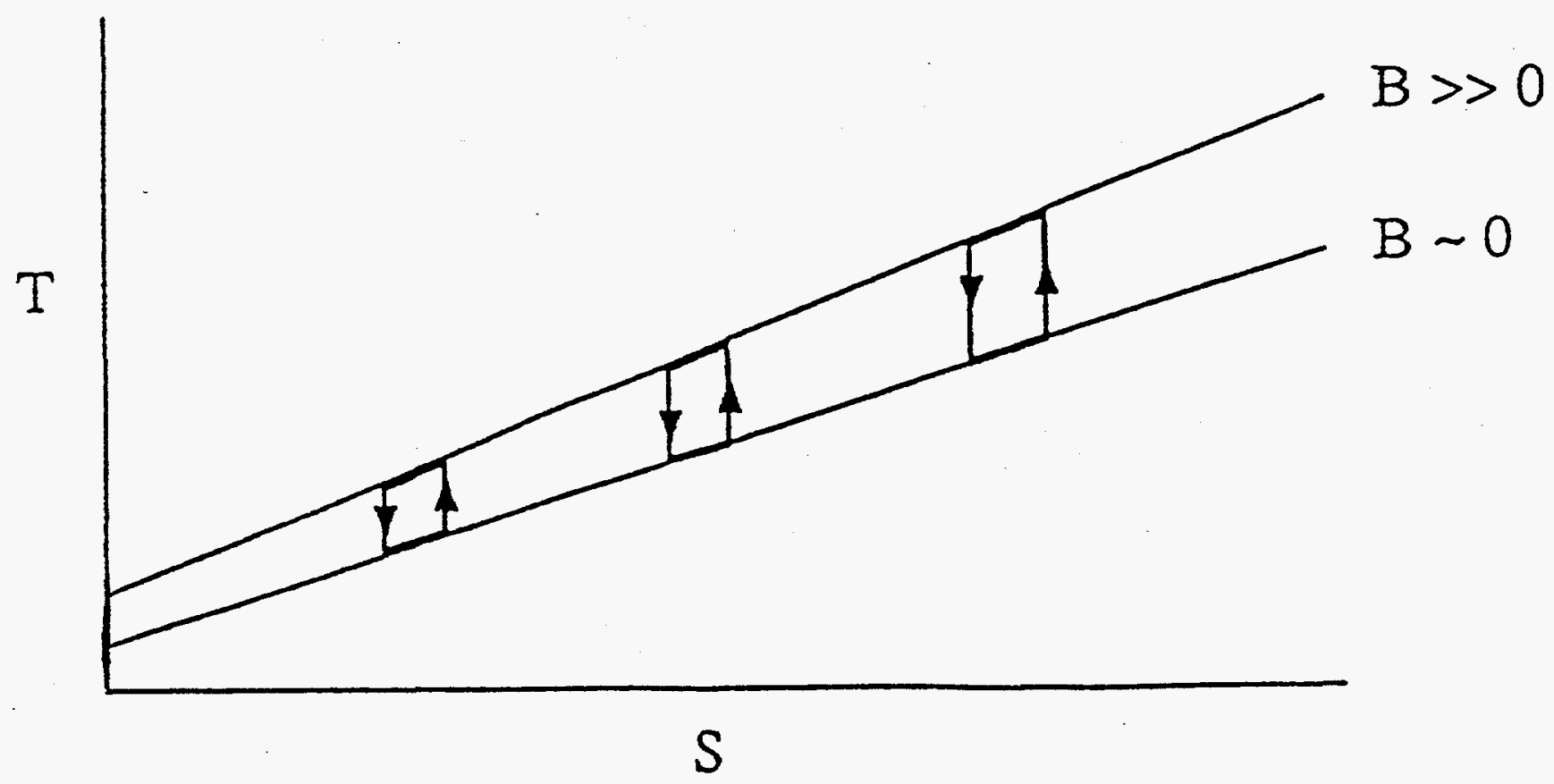

Figure 2. T-S Diagram of Ideal Ferromagnetic Refrigerant for an AMR Cycle 
Analysis of the AMR cycle as applied to the liquefier design shows that the temperature dependence of the ideal material's adiabatic temperature change depends on the extent of the flow imbalance in the matrix. Figure 3 shows the temperature dependence of the adiabatic temperature change of materials ideally suited for several different flow imbalances. Because the ideal material properties depend on the flow imbalance, selection of an appropriate magnetic material for liquefier operation is coupled to details of the liquefier design.

Because the adiabatic temperature change of available magnetic materials is small relative to the total span of the magnetic liquefier, a two-stage design was chosen. The upper stage operates from $77 \mathrm{~K}$ to about $40 \mathrm{~K}$ and the lower stage operates from $40 \mathrm{~K}$ to approximately $20 \mathrm{~K}$. More design stages can result in greater efficiency, but at an increase in system complexity and cost. Many materials were considered for use. The ideal refrigerant material $\mathrm{T}-\mathrm{S}$ curves were used as guides in choosing materials that would give high refrigerator efficiency. Also, the thermal and mechanical properties of the materials were assessed to assure good matrix performance as a regenerator. For the upper stage, $\mathrm{GdNi}_{2}$ was identified as an appropriate material for high efficiency operation and, for the lower stage, GdPd was chosen.

Regenerator properties of the AMR matrix have also been characterized. Engineering correlations provide values of the heat transfer coefficient between the bed and heat transfer fluid. Similarly, pressure drops, longitudinal thermal conduction, and axial dispersion in the bed can be calculated from standard correlations. Some competing effects are evident. Excellent heat transfer requires large surface areas, which, in turn, suggests that bed particles should be of small diameter. Flow pressure drop is less for flow through beds of large diameter particles. Pressure drops across beds of short length are smaller than those across longer beds, but longitudinal thermal conduction for short beds is greater. Because of the importance of effective heat transfer for efficient liquefier operation, heat transfer effects were paramount and short beds of small diameter particles were the design target. Key design parameters such as particle size, bed dimensions, and cycle period were determined by a numerical model developed for analysis of the AMR refrigerator and adapted for use in liquefier analysis. Using this model, $5 \mathrm{~cm}$ long particle beds of $0.15 \mathrm{~mm}$ diameter spheres were chosen for a liquefier operating with a 2 s period.

First and second stage helium mass flow rates were determined through a process that began with a specification of the cold helium mass flow rate in the counterflow heat exchanger required to liquefy the incoming hydrogen stream. For the subscale device, the average mass flow rate required is $5 \mathrm{~g} / \mathrm{s}$. The cold outlet flow from the second stage of the magnetic liquefier is split between this flow and a flow that is returned through the AMR bed. The required return flow through both stages of the AMR is determined by irreversibilities and inefficiencies in the system that contribute to the thermal load that must be transported to the liquid nitrogen heat sink. 


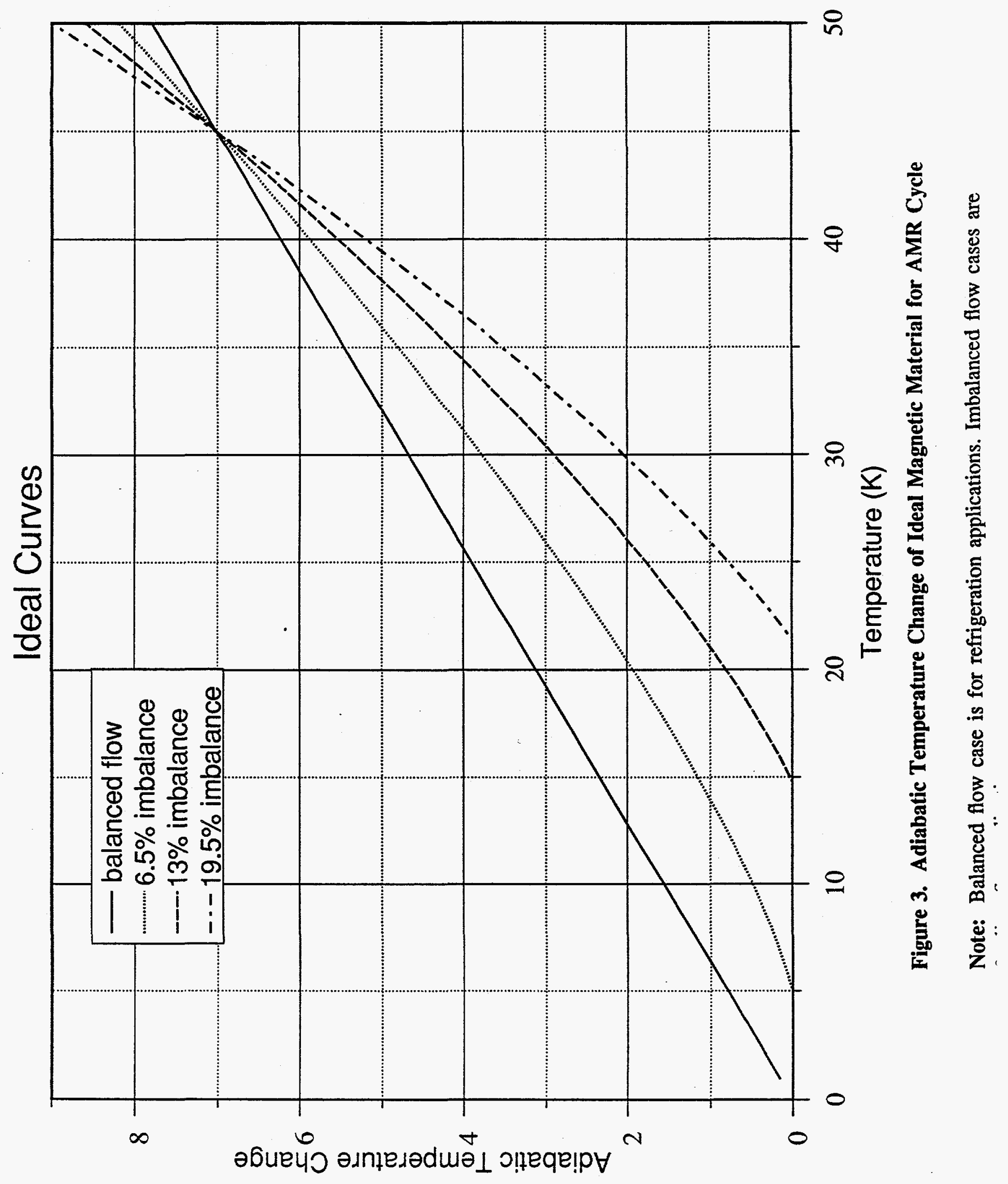




\subsection{Magnetic Liquefier Schematic}

Figure 4 is a schematic of the two-stage magnetic liquefier. Each stage consists of two stationary AMR beds in linear alignment with a solenoidal magnet that moves over the beds in reciprocating linear motion. With this configuration, the two beds in a stage are always out of phase by one-half cycle. If one bed has just been magnetized, the other has just been demagnetized, or if one bed is experiencing flow from its cold to its hot end, the other is experiencing flow from its hot to its cold end. With a total operating period of $2 \mathrm{~s}$, the cycle is divided into two $0.5 \mathrm{~s}$ segments for magnet motion, separated by two $0.5 \mathrm{~s}$ segments for helium flow through the beds and into the counterflow heat exchanger.

This configuration has several advantages. All helium flow paths are stationary, so moving seals are not required. In addition, as the magnet moves, the attractive force on the magnet winding caused by the magnetic material within its bore is partially balanced by the attractive force caused by the material in the second set of beds toward which the magnet is moving. Because these forces are quite large, balancing reduces the size of the system support structure required. This configuration is also similar to an existing laboratory AMR. Experience gained in the fabrication and testing of that device reduces the risk of this first-of-a-kind liquefier.

The schematic shows the system immediately after the magnets moved from the upper to the lower beds, and valves A and B were set to Position 1. Because of the magnet motion, the upper beds were demagnetized and cooled, and the lower beds were magnetized and warmed. With the valves in Position 1, helium flows in the upper first stage bed from the $77 \mathrm{~K}$ high temperature end to the $39 \mathrm{~K}$ low temperature end. The outlet flow from the upper first stage bed is split at a plumbing tee so that $80 \%$ of the outlet flow continues to the lower first stage bed and the remainder is diverted to the upper second stage bed. The flow split is determined by the flow impedance presented by the proportioning valve $\mathrm{PV}_{\mathrm{b}}$. Flow to the lower first stage bed is warmed to about $80 \mathrm{~K}$ as it passes from the bed's lower temperature end to its higher temperature end. This flow, emerging from the higher temperature end of the lower first stage bed, passes through the three-way valve A in Position 1, and continues to the helium circulation pump where the flow cycle begins again.

The flow diverted to the upper second stage bed enters the bed at its $39 \mathrm{~K}$ high temperature, passes through the bed, and emerges cooled to about $19 \mathrm{~K}$. The $19 \mathrm{~K}$ helium flow from the second stage upper bed is split at a tee. Proportioning valve $\mathrm{PV}_{\mathrm{a}}$ is adjusted so that about $10 \mathrm{~g} / \mathrm{s}$ of the emerging cold helium flow passes through the hydrogen-helium counterflow heat exchanger and the remainder is diverted to the magnetized lower second stage bed, where it is warmed to about $45 \mathrm{~K}$ before it flows to the lower bed of the first stage for additional warming to about $80 \mathrm{~K}$. 


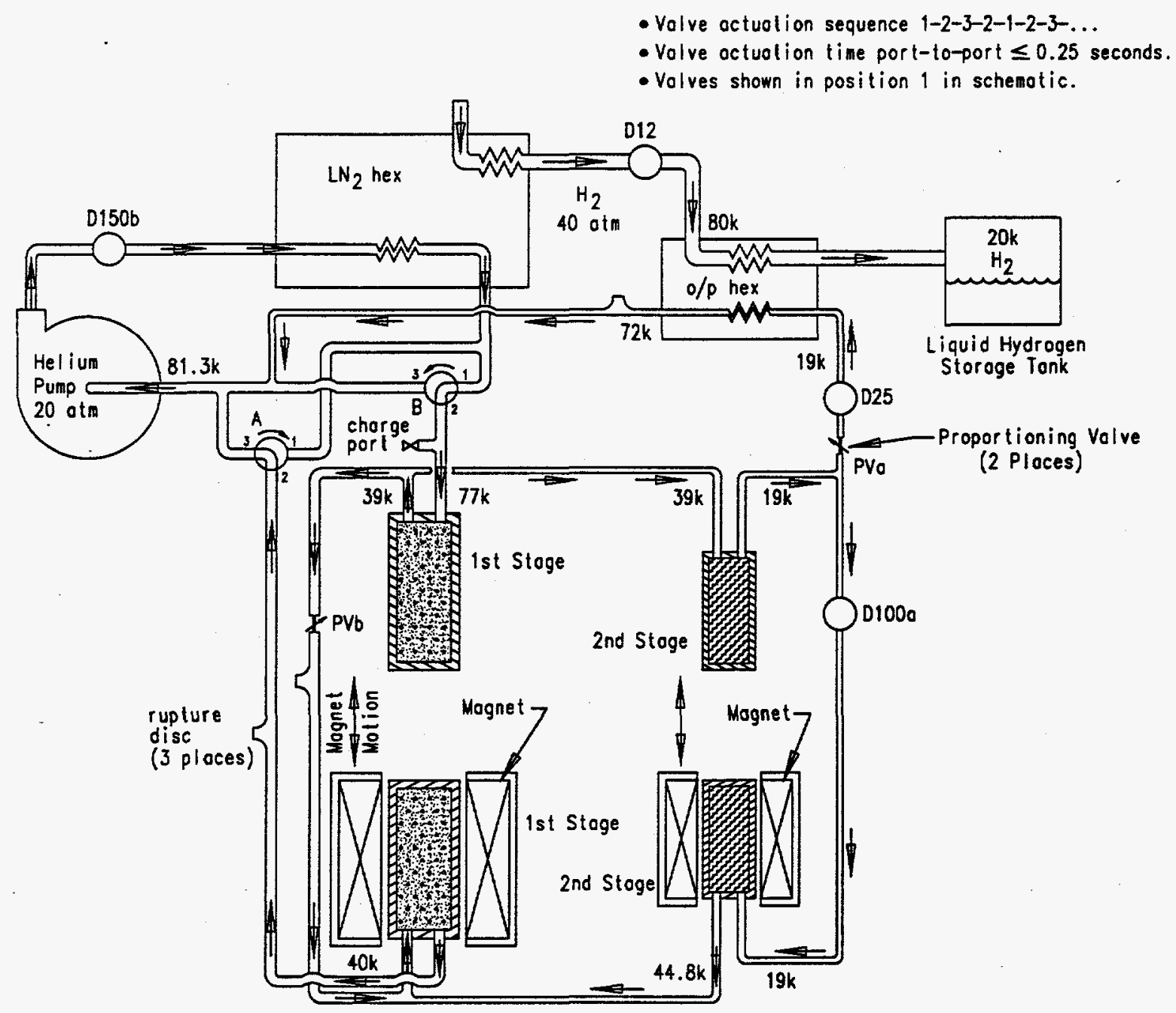

Volve Positions For Three-Woy Valves

Position 1

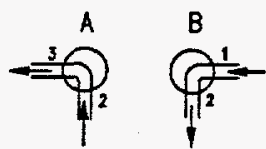

Position 2

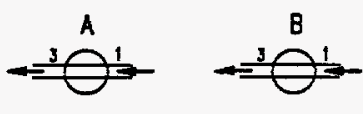

Position 3

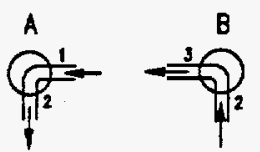

Figure 4. Flow Schematic for Two-Stage Hydrogen Liquefier 
After $0.5 \mathrm{~s}$ of flow as described above, valves $\mathrm{A}$ and $\mathrm{B}$ are put into Position 2, a dwell position. The valves are left in this position for $0.5 \mathrm{~s}$, during which helium flow from the helium circulation pump bypasses the beds and flows directly back to the pump. Under these conditions there is also no helium flow into the hydrogen-helium heat exchanger. During this bypassed flow period, the first and second stage magnets move off the lower beds onto the upper beds. Valves A and B are then put into Position 3, and flow begins again, with the upper and lower beds in each stage reversing roles from those described previously. The average mass flow rate over a cycle, under these operating conditions, is $5 \mathrm{~g} / \mathrm{s}$.

\subsection{Liquefier System Configuration}

Figure 5 is a sketch of the liquefier system key components. The design is an assembly of seven subsystems:

- the AMR regenerator beds,

- the reciprocating magnet drive,

- first and second stage superconducting magnet systems, thermal shields, and liquid helium vessels,

- the support structure of the beds and magnets,

- the vacuum system-vacuum vessel (cold box), and pumps,

- fluid pumping, piping, and flow control system, and

- the computerized control system.

Several subsystems, and components of others, will be designed by specialized manufacturers to ACA-supplied specifications. These include: the drive system; the superconducting magnet system; vacuum vessel, vacuum system pumps and gauges; heat exchangers; and the pump/gauges for the helium flow system. Other subsystems are unique to the magnetic liquefier and they will be completely designed and assembled by ACA. The most important of these are the AMR regenerator beds and the liquefier control system. Other components that will require special design are the support structure which support the beds and on which the magnets ride, and the sensor system that monitors performance and provides data for control system operation. 


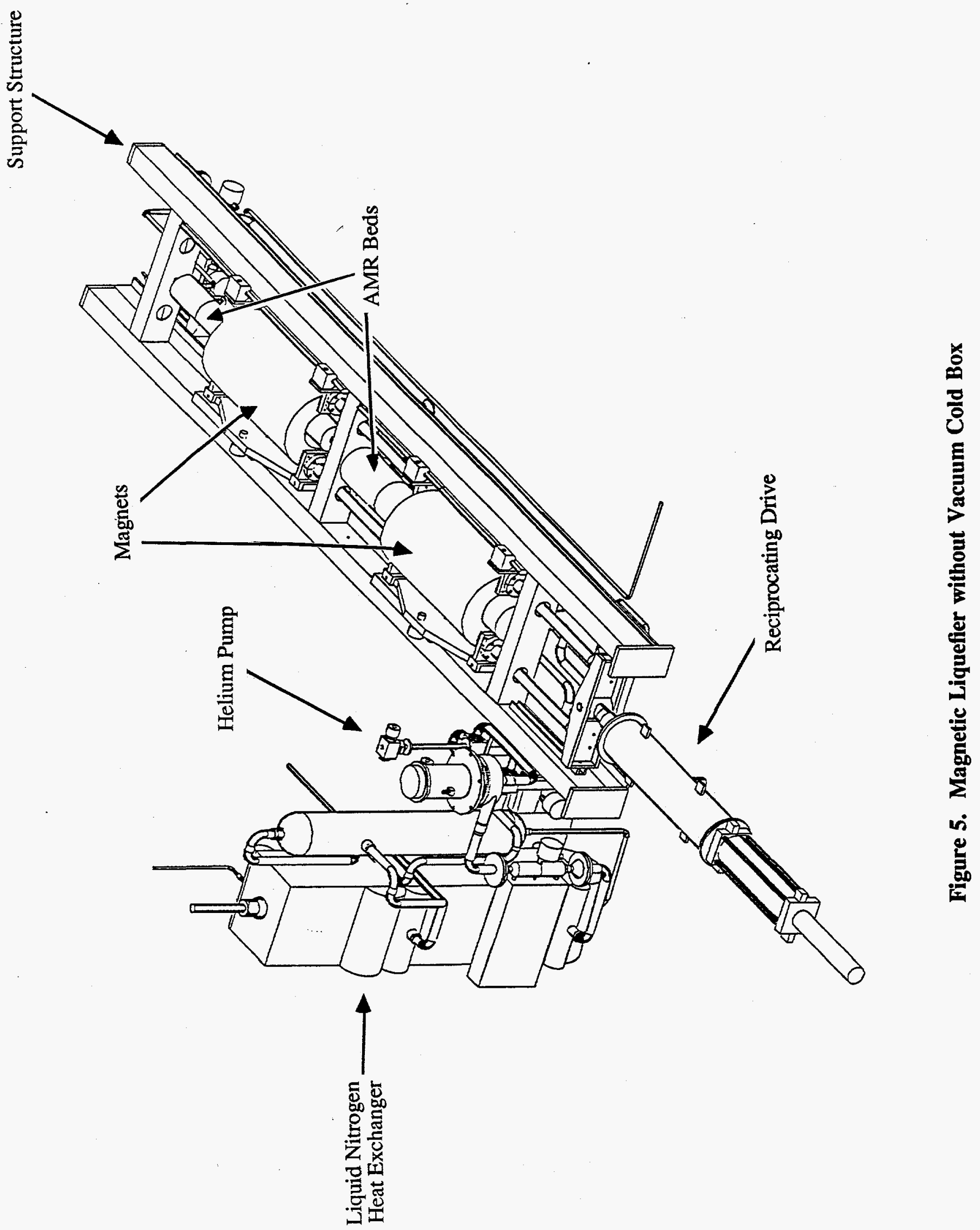




\subsubsection{Magnet Subsystem}

The superconducting magnet system is unusual. It consists of two identical superconducting solenoids immersed in liquid helium-containing cylindrical vessels. The vessels are interconnected by rigid composite rods and mounted on bearings so the two magnet systems can be moved over the beds in phase. A $77 \mathrm{~K}$ thermal shield (not shown in Figure 5) surrounds the magnet helium vessels, regenerator beds, and tracks on which the magnets move. The moving helium vessels are connected to a stationary helium storage dewar (not shown) by a flexible helium transfer line so the liquid helium level at the magnets can be maintained without interrupting operation. The magnets will be stationary while they are charged and put in persistent mode. After charging, the leads will be disconnected, and the magnet system will be put in motion. The magnet system can operate continuously for several months without recharging.

\subsubsection{AMR Regenerator Bed Design}

The AMR beds are designed to operate in the presence of large changing magnetic fields, at temperatures between $77 \mathrm{~K}$ and $20 \mathrm{~K}$ with 40 atm helium flowing through them. The beds will be fabricated of cryogenic grade G-10, a nonconducting fiberglass epoxy composite, to eliminate eddy current heating. Epoxy bonds, tested at operating temperatures, will be used between bed components. Pressure drops in the beds are minimized by careful flow channel design. The assembly is configured for easy installation and removal of the beds for maintenance.

\subsubsection{Support Structure and Drive Design}

The magnet system and beds are supported by a structure of rectangular stainless steel tubing which is flooded with liquid nitrogen to provide a thermally stable operating environment. The magnet supporting and guiding bearings ride in this support structure.

A drive system external to the vacuum vessel is coupled to the magnet system by a hermetically-sealed reciprocating drive shaft. The drive system is hydraulically driven and a programmable servo system will be used to control it.

\subsubsection{Vacuum System and Fluid System}

All components of the liquefier operating below ambient temperatures will be within a vacuum vessel. A pumping system capable of evacuating this system within a few hours will be installed. A large roughing pump will evacuate the system to a pressure where the low pressure turbomolecular pump can maintain the $10^{-6}$ torr 
operating pressure.

The piping and pumping system for circulating the helium for AMR operation will be contained within the vacuum chamber. The piping will be stainless steel tubing with all welded joints. A commercial pump capable of circulating $77 \mathrm{~K}$ helium at greater than $500 \mathrm{~g} / \mathrm{s}$ with a pressure drop of about 1.5 atm will be included. Solenoid-actuated three-way valves will control the helium flow in the system during different parts of the cycle.

\subsubsection{Computer Control System}

AMR cycle operation requires a coordinated magnet motion - fluid flow control system. The control system must ensure safe operation, monitor and control the hydrogen stream flow rate, temperature and liquefaction rate, and monitor/control liquid nitrogen and liquid helium cryogen levels. Thus, multiple control loops are required and many temperature, flow rate, pressure, position and load sensors must be monitored to provide the necessary information. For this reason, the control system and data acquisition system for the liquefier presents a complex, tightly-coupled design task for test and control system engineers. More than 100 sensors are included in the design.

\section{Alternative Magnetic Refrigerator Concepts}

Several magnetic refrigeration concepts were investigated for potential liquefaction devices. Besides the Active Magnetic Regenerator (AMR) cycle, other concepts studied were: an external regenerative cycle, an internal regenerative cycle, and a recuperative cycle. A complete analysis of the AMR concept is presented in Section 3 ; in this section analysis of three alternative concepts is discussed. See Table 1. Each concept has advantages and disadvantages. For application to hydrogen liquefaction, each of the alternative concepts had disadvantages that eliminated them as a basis for a magnetic hydrogen liquefier.

Table 1. Alternative Concepts

External Regenerator Internal Regenerator Recuperative
Moving Material Moving Material Moving Material
Stationary Magnet

Moving Magnet Stationary Magnet
Brayton Cycle Brayton Cycle Brayton Cycle 


\subsection{External Regenerator Magnetic Refrigerator}

A direct magnetic analog of the regenerative gas Brayton cycle refrigerator is shown in Figure 6. This Magnetic Refrigerator (MR) concept involves a reciprocating ferromagnetic material and a superconducting circular solenoid magnet. Other options for Brayton cycle operation include reciprocating and charge/discharge magnets.

This MR works as follows: the magnetic material is magnetized and its temperature is raised from $\mathrm{T}_{h}$ to $\mathrm{T}_{h}+\Delta \mathrm{T}_{h}$ (see the Temperature-Entropy (T-S) diagram in Figure 7). The heat of magnetization is then removed by circulating gaseous helium, (GHe) through the magnetic material in series with the Hot Heat Exchanger (HHEX), which reduces the temperature of the magnetic material to approximately $\mathrm{T}_{h}$. Then the first regenerative process is executed by circulating $\mathrm{GHe}$ through the external regenerator as the regenerator duct is moved from top to bottom. The large thermal mass of the regenerator cools the magnetized material to near $\mathrm{T}_{c}$. After regeneration to near $\mathrm{T}_{c}$, the magnetic material is demagnetized by removing it from the magnet, which cools the material to $\mathrm{T}_{c}-\Delta \mathrm{T}_{c}$. Helium gas is circulated through the Cold Heat Exchanger (CHEX) and the magnetic material to remove heat from a load and warm the magnetic material back to near $T$. The reverse regenerative process is performed while the regenerative duct is moved from the bottom to the top of the regenerator while GHe is circulated. This process heats the magnetic material from about $T_{c}$ to near $T_{h}$, which completes the cycle. This option requires three flow loops: one with a pump to circulate GHe through the sink and the magnetic material, another with a pump to circulate GHe through the load and magnetic material, and a third with a displacer that moves back and forth to move the GHe though the external regenerator and the magnetic material.

The T-S diagram in Figure 7 shows the cooling power $\mathrm{Q}_{c}$, the heat rejected $\mathrm{Q}_{h}$, and the heat transferred to and from the regenerator $\mathrm{Q}_{r}$ for an ideal Brayton cycle magnetic refrigerator. A characteristic of a Brayton cycle spanning a temperature range that is large compared to the adiabatic temperature of the magnetic material is that $\mathrm{Q}_{r}$ can be much larger than $\mathrm{Q}_{c}$. 


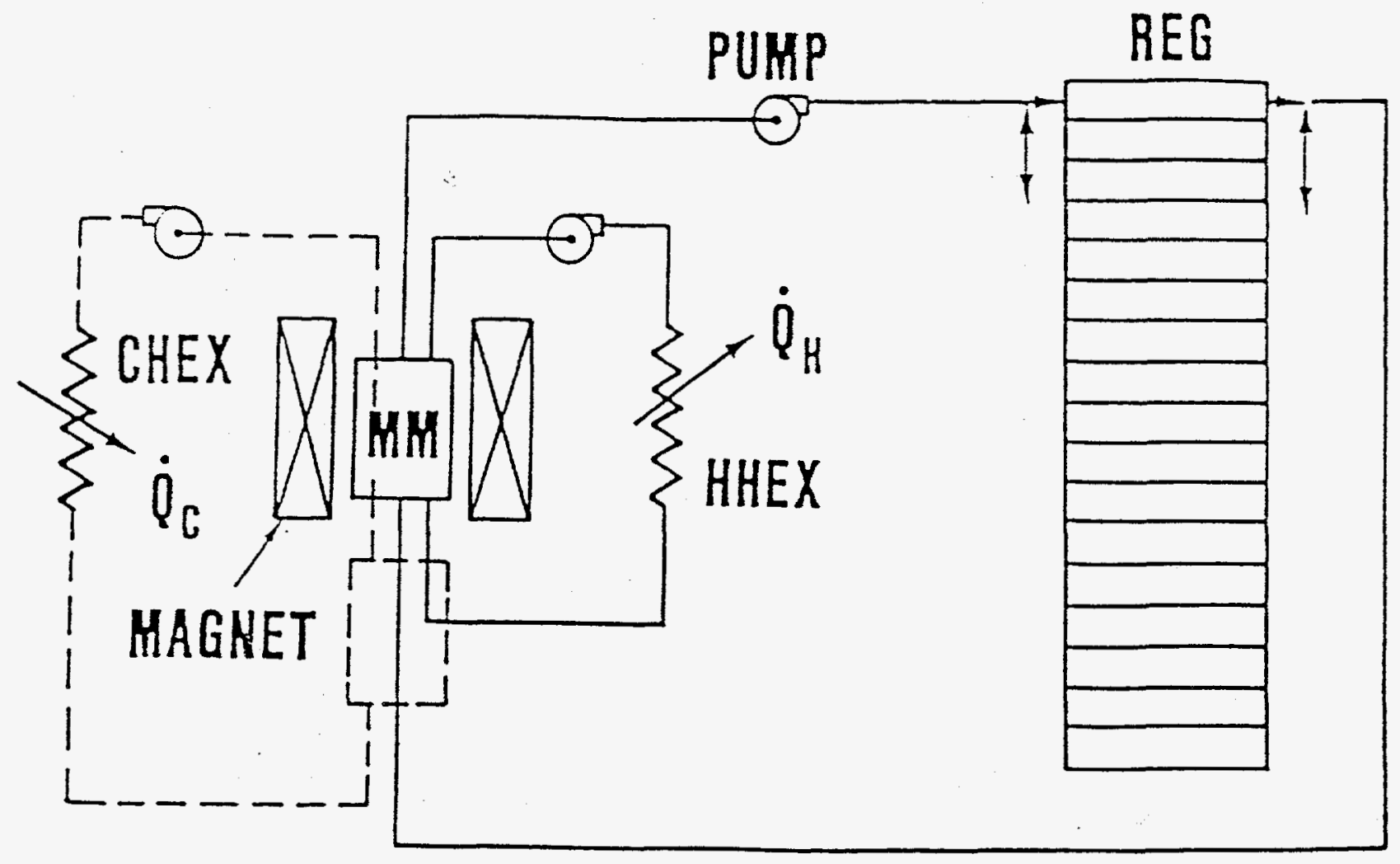

Figure 6. Magnetic Refrigerator using External Solid Regenerator 


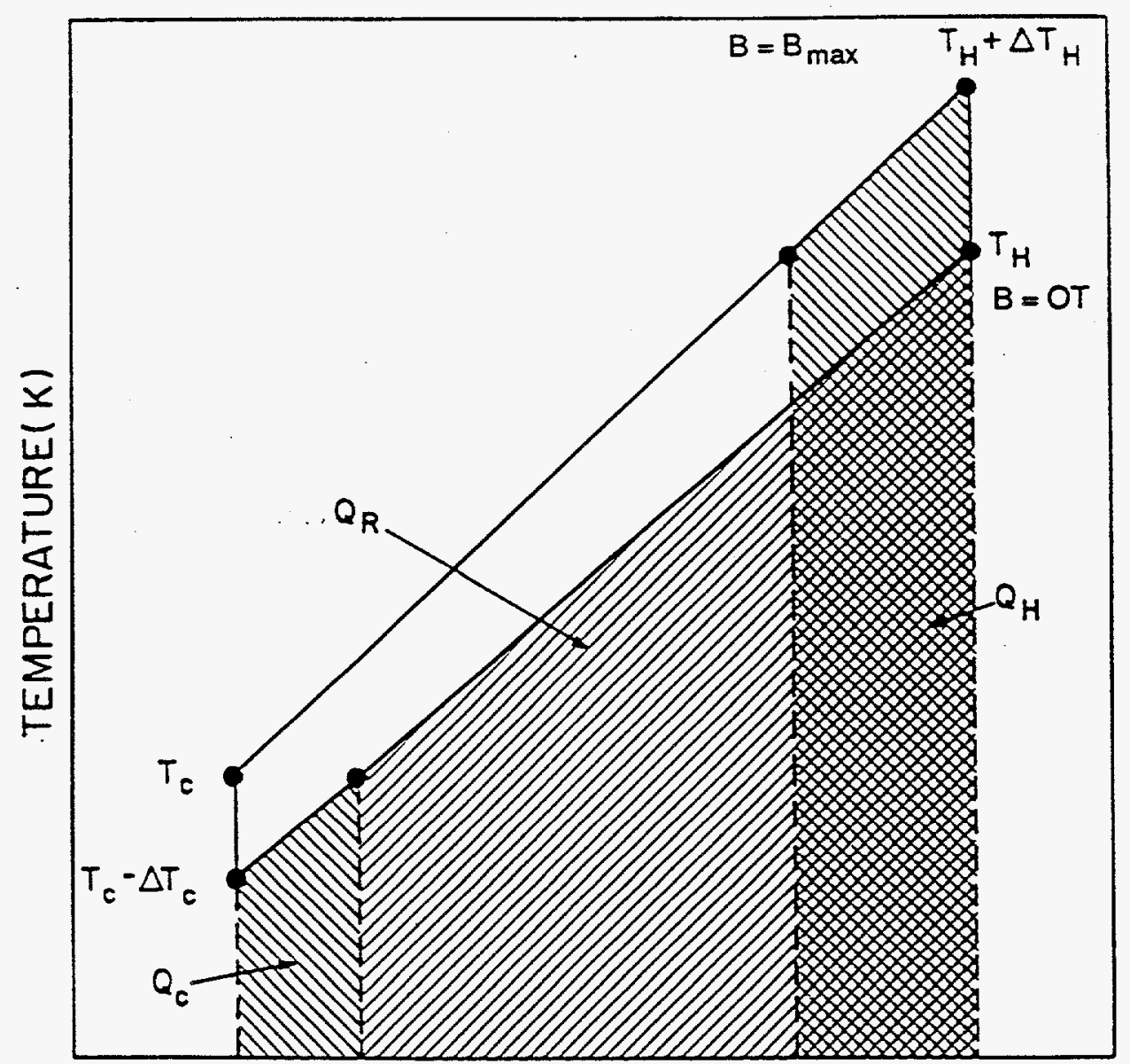

ENTROPY $(\mathrm{J} / \mathrm{kg} \cdot \mathrm{K})$

Figure 7. Temperature-Entropy Diagram for Brayton-Cycle MR 
To avoid large thermal variations during regeneration of the magnetic material, the external regenerator must have a thermal mass at least 20 times that of the magnetic material. This ensures temperature stability of the external regenerator. The regenerator is cycled back and forth so that the regenerator temperature profile is maintained in steady state operation. The regenerator temperature is near $T_{h}$ at the top and $\mathrm{T}_{c}$ at the bottom.

Disadvantages of this concept include: the large GHe flow rate required to thermally connect the external regenerator to the magnetic material, the large regenerator mass required, and the pump temperature cycling, plumbing, and helium gas connecting the magnetic material and regenerator.

Preliminary sizing of the $60 \mathrm{~K}$ to $77 \mathrm{~K}$ upper liquefier stage was performed to investigate design feasibility for the large cooling power required. It was assumed that this $60 \mathrm{~K}$ to $77 \mathrm{~K}$ stage would be the upper stage of a three-stage 0.1 ton/day magnetic liquefier, spanning a $20 \mathrm{~K}$ to $77 \mathrm{~K}$ temperature range, and that the lower two stages had $50 \%$ efficiencies with respect to Carnot. Given these conditions, the upper stage would need to provide $5900 \mathrm{~W}$ of cooling at $60 \mathrm{~K}$.

Figure 8 shows a calculated T-S diagram for $\mathrm{GdNi}$, a candidate magnetic material for a regenerative Brayton magnetic refrigerator in this temperature range. For this analysis, it was assumed that a field of 7 Tesla is the maximum magnetic field. The cycle shown on the T-S diagram represents an ideal cycle with perfect heat exchange with the hot and cold ends and perfect regeneration. The maximum cooling power achievable per unit mass of $\mathrm{GdNi}$ per cycle is $1.11 \mathrm{~J} / \mathrm{g}$. For a real cycle, it is assumed that the useful cooling power obtained would be one-half of the maximum cooling power because of heat transfer inefficiencies and heat leaks within the refrigerator. Assuming a cycle could be completed in $10 \mathrm{~s}, 105 \mathrm{~kg}$ of $\mathrm{GdNi}$ would be required to provide the required cooling power at $60 \mathrm{~K}$ for the 0.1 ton/day liquefier.

For $105 \mathrm{~kg}$ of $\mathrm{GdNi}$, the external regenerator must have a mass of at least 3500 $\mathrm{kg}$ of lead if temperature swings in the regenerator are less than $1 \mathrm{~K}$, as desired. For different cycle times with the same cooling power, the mass of the magnetic material and the regenerator scale linearly with cycle time. 


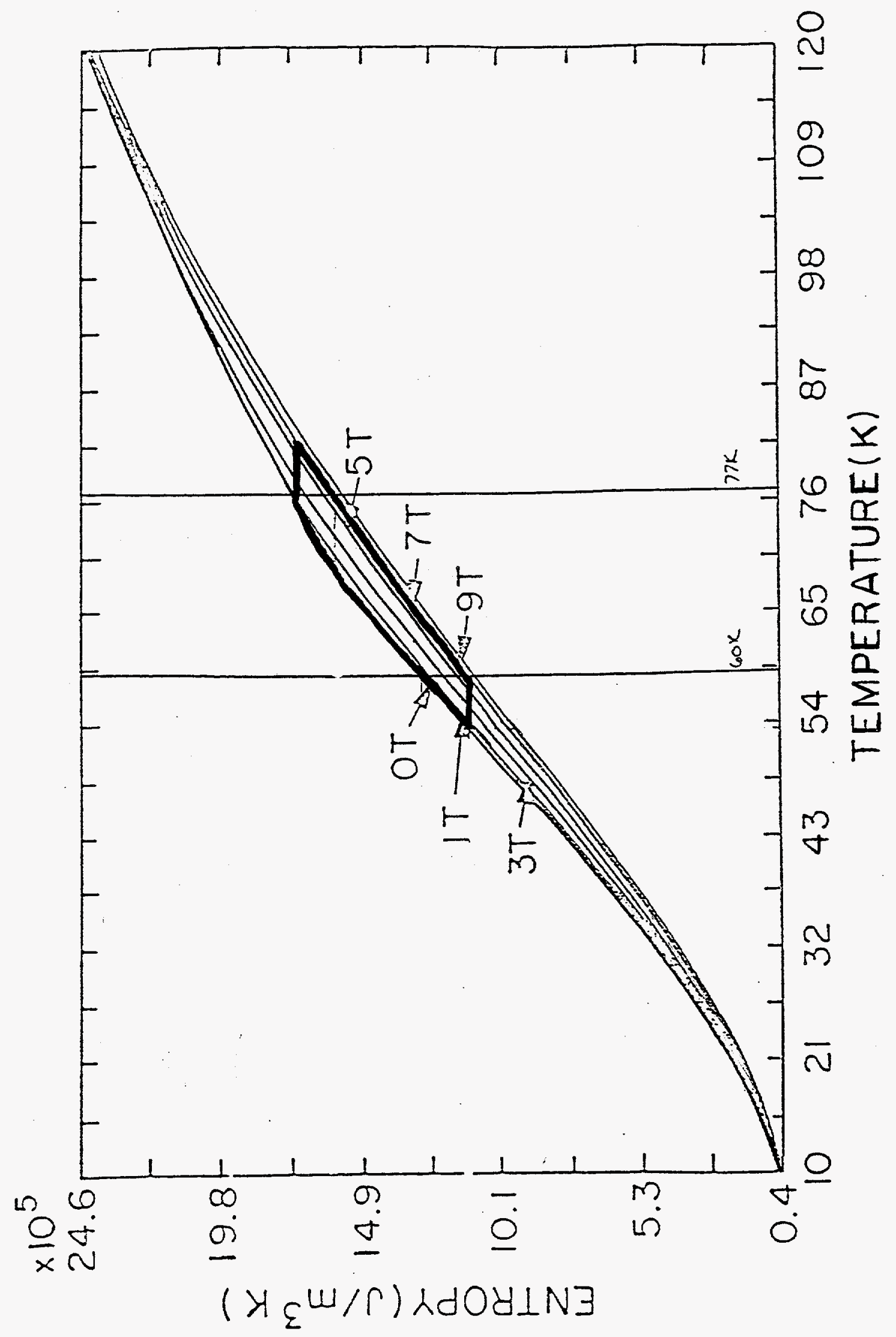

Figure 8. Calculated Temperature-Entropy Diagram for GdNi 
The mass flow rate of the GHe required to perform the external regeneration parts of the cycle is $21 \mathrm{~kg} / \mathrm{second}$. At $1 \mathrm{MPa}(10 \mathrm{~atm})$ mean pressure, this mass flow rate corresponds to a volume flow rate of $3 \mathrm{~m}^{3} / \mathrm{s}$ at $70 \mathrm{~K}$. The pumping losses will be significant unless the piping diameter is large.

The conclusion of this preliminary analysis is that an external regenerator reciprocating magnetic refrigerator is possible but the problems of the large helium flow and the large mass of regenerator material, combined with difficult flow control, make this concept less favorable than the AMR concept chosen.

\subsection{Internal Regenerator Magnetic Refrigerator}

One problem with the external regenerator concept is the large flow between the magnetic material and the regenerator required to effect the heat transfer. Use of an internal solid regenerator could ameliorate this problem.

This MR, operating on a Brayton cycle with reciprocating ferromagnetic material, internal solid regeneration, and a reciprocating circular solenoid is shown schematically in Figure 9. The refrigerator works as follows: consider sheets of magnetic material in the bore of a magnet, as shown in part $\mathrm{A}$ of the figure. The material will be at $\mathrm{T}_{h}$ $+\Delta \mathrm{T}_{h}$ after the magnetization. A flow of fluid through the magnetized material and the HHEX (not shown) will reduce the temperature to $T_{h}$. Now the regenerator, also in sheets, is moved through the magnetic material (diagram B). The exchange gas and close tolerances between the magnetic and regenerator sheets will enhance heat transfer to cool the magnetic material from $\mathrm{T}_{h}$ to $\mathrm{T}_{c}$ as in diagram C. At this point the magnetic material is demagnetized (diagram $D$ ), thereby cooling it from $T_{c}$ to $T_{c}-\Delta T_{c}$. After heat transfer at a CHEX (not shown), regeneration again takes place by movement of the regenerator sheets back through the magnetic material. This process warms the magnetic material from $\mathrm{T}_{c}$ to $\mathrm{T}_{h}$, at which point the magnetic material can be magnetized again, starting a new cycle. The T-S diagram for an ideal ferromagnetic material in Figure 9 also shows the path of the cycle.

The advantage of this concept over the external solid regenerator is that fluid flow connecting the magnetic material and regenerator is replaced by regenerator movement through the magnetic material. Unfortunately there are several disadvantages to this concept. The first is that the heat transfer requirements for regeneration require a contact area per unit volume of the magnetic material and regenerator that is impractical to manufacture. For a thermodynamic cycle based on the configuration described in the previous section on external regenerative cycles ( 0.1 ton/day liquefier upper stage, spanning $60 \mathrm{~K}$ to $77 \mathrm{~K}$, with a cycle time of $10 \mathrm{~s}$ ) we have the following conditions:

- $\quad 3.5 \mathrm{~s}$ allowed for regenerative heat transfer 


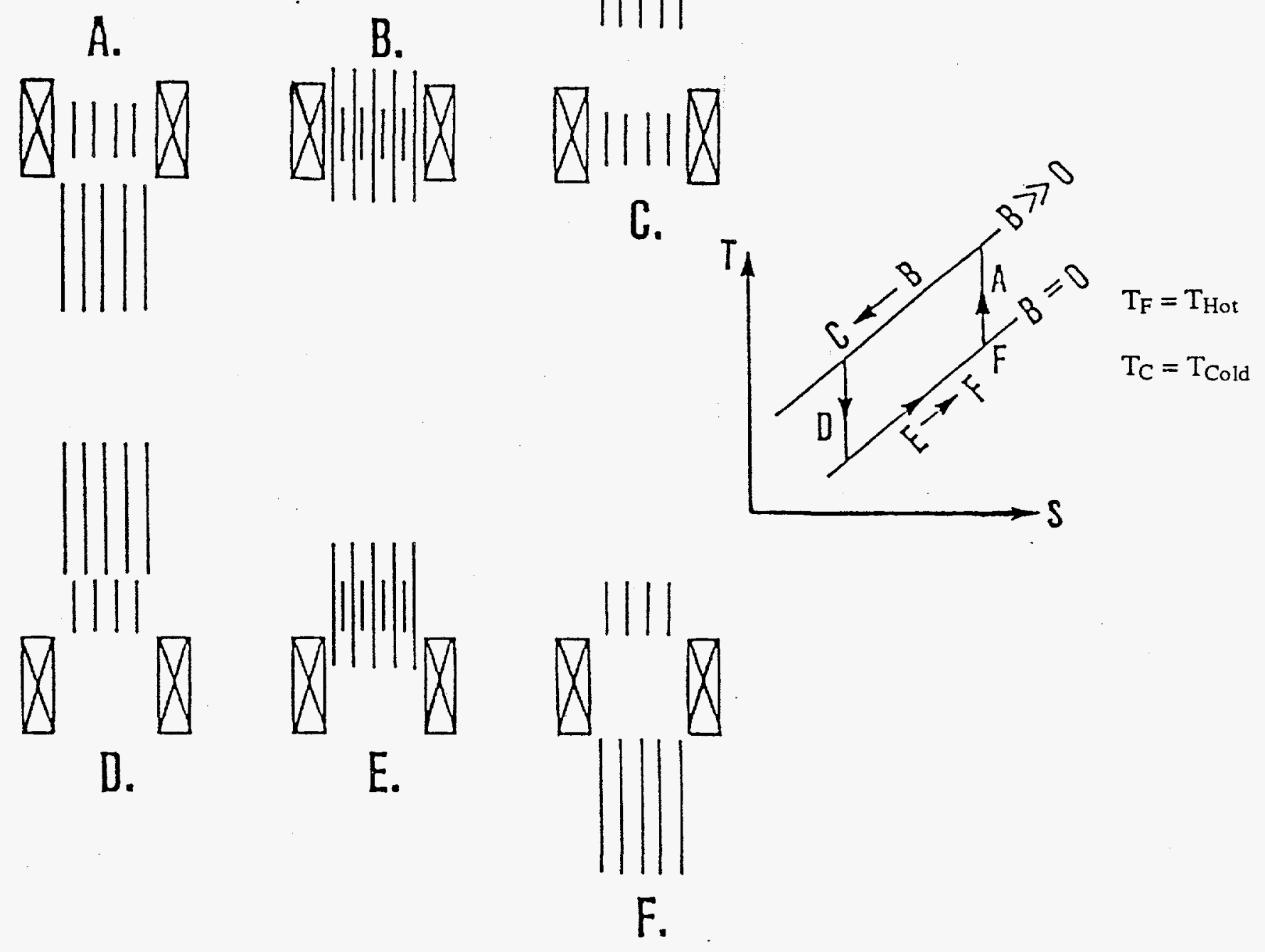

Figure 9. Schematic of Internal Regenerative Brayton Cycle Magnetic Refrigerator 
- regenerative heat transfer rate: $11 \mathrm{~kW}$

- $0.011 \mathrm{~m}^{3}$ of magnetic material

- $0.32 \mathrm{~m}^{3}$ of regenerator material (lead)

- $1 \mathrm{~K}$ temperature difference between magnetic material and the regenerator

- heat transfer gap between the magnetic material and the regenerator: $0.076 \mathrm{~mm}$

Using the above information, and assuming the magnetic material travels five times its own length during the regenerative part of the cycle, geometry dictates that the lead regenerator sheets shown in Figure 9 must be $0.98 \mathrm{~mm}$ thick to obtain the necessary heat transfer area. A typical arrangement may be 50 sheets each $1 \mathrm{~m} \mathrm{X} 6.5$ $\mathrm{m} \mathrm{X} 0.98 \mathrm{~mm}$. The corresponding geometry of the magnetic material would be 50 sheets each $1 \mathrm{~m} \mathrm{X} 1.3 \mathrm{~m} \mathrm{X} 0.17 \mathrm{~mm}$. The thickness dimension of these configurations does not vary with cycle time.

This configuration is impractical from a manufacturing standpoint, particularly when the large forces which act on the magnetic material are considered. An additional disadvantage is that the magnet system must be sized to contain both the magnetic material, regenerator, and support structure. This increases the volume of the required field region by a factor of at least six, based on magnetic material and internal regenerator volumes.

\subsection{Recuperative Magnetic Refrigerator}

An alternative to using regenerative cycles is the recuperative cycle. (Regenerative refers to periodic heat flow and recuperative refers to continuous heat flow.) Instead of heat transferred from the magnetic material to a regenerator via the working fluid, as in the two concepts discussed above, in a recuperative cycle the heat is transferred to the working fluid as the magnetic material is cooled down to $\mathrm{T}_{c}$. Later in the cycle, the same quantity of heat is transferred back to the material from the working fluid, heating the material back up to $\mathrm{T}_{h}$. Thus the recuperative cycle differs from the regenerative cycle because the heat transfer is "internal" and leads to a steady state temperature distribution in both the magnetic material and the working fluid. In a regenerative cycle, the heat transfer is "external" because the heat is stored in the regenerator material, which generally leads to a time-dependent temperature profile in the regenerator.

There are two cycles suitable for a recuperative magnetic refrigerator: the Brayton cycle and the Ericsson cycle. A general schematic of a Brayton cycle, wheel-type magnetic refrigerator is shown in Figure 10. Note the counterflow arrangement of the working fluid and rotating magnetic material. The difference between the Brayton and Ericsson cycles is the isentropic magnetization and demagnetization steps in the Brayton cycle; that is, the working fluid is isolated from the magnetic material. In the Ericsson cycle, the magnetization and demagnetization steps take place isothermally, 
with the working fluid in contact with the magnetic material.

A rotary Brayton cycle recuperative magnetic refrigerator was modeled. Three separate stages, as shown in Figure 10 were arranged in series to span the $20 \mathrm{~K}$ to 80 $\mathrm{K}$ temperature range. Each stage spanned approximately $20 \mathrm{~K}$. The principal difference between the stages was the magnetic material chosen. From our previous work it is known that the T-S lines should either converge as the temperature decreases for optimal efficiency or be approximately parallel. ${ }^{11}$ This indicates the magnetic material should be chosen so that its Curie temperature is near $\mathrm{T}_{h}$. An example of material with these T-S properties is $\mathrm{GdNi}$ (Figure 8). The Curie temperature of GdNi is $70 \mathrm{~K}$.

The materials chosen for the model stages are from the $\mathrm{Er}_{x} \mathrm{Gd}_{1-x} \mathrm{Al}_{2}$ series of alloys. ${ }^{14}$ The entropy and heat capacity data was generated by the MATFCN program which uses molecular field theory and standard thermodynamic relationships to compute these quantities. Unfortunately, experimental results ${ }^{2}$ indicate that actual material properties of this series of alloys is quite different than predicted values from MATFCN. However, until suitable magnetic materials are chosen, the MATFCN results can be used as examples of "real" materials as opposed to "ideal" materials. For this study, materials with Curie temperatures of $40 \mathrm{~K}, 60 \mathrm{~K}$, and $80 \mathrm{~K}$ were used in the model stages.

The choice of working fluid is easy for the lowest stage: gaseous helium is the only appropriate choice to avoid two-phase flow. For the upper stages more latitude exists, but gaseous helium was chosen as the working fluid there, as well.

One of the most important design features of a recuperative magnetic refrigerator is the heat transfer geometry. Two basic geometries have been considered: parallel plates and packed beds. In a rotary device, parallel plates offer a simple solution to the helium flow control problem, although there can be a problem with obtaining enough heat transfer area per volume of the magnetic material. Briefly, the flow control problem arises in the magnetization and demagnetization steps where the magnetic material must be separated from the working fluid so the fluid can pass through the external heat exchangers. With parallel plates, the flow control can be managed by "extractors" which fill the helium flow path, forcing the gas to flow through the heat exchangers. Packed beds offer a way to meet the heat transfer area requirement, but present a much more challenging flow problem which we have not resolved.

A parallel plate geometry is implicit in the model. The heat transfer geometry is completely specified by several parameters:

- the cross-sectional area of the magnetic material (the area perpendicular to the direction of movement); 


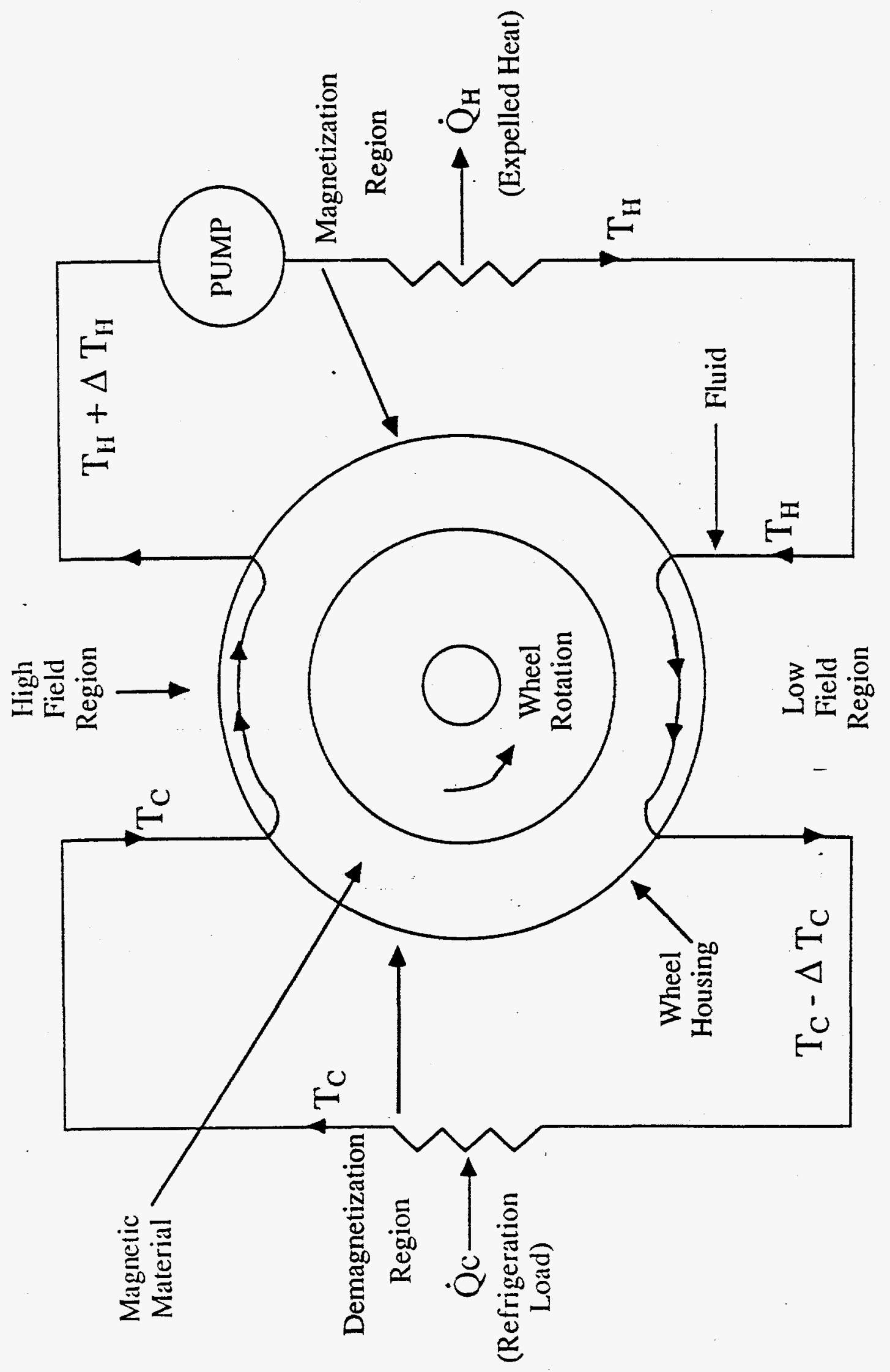

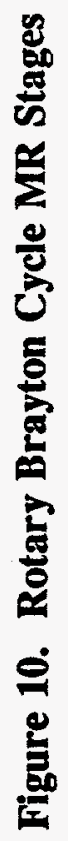


- the related cross-sectional area of the helium flow path;

- either the hydraulic or heated radius;

- either the length of the recuperative section or the wheel circumference.

These parameters can be converted into parallel plate parameters (such as the number, thickness, length, and spacing of the plates) but they also allow comparisons with other geometries such as packed beds.

Performance of the three-stage, Brayton cycle magnetic liquefier was analyzed using the MITAS ${ }^{3}$ thermal analysis program. MITAS is a lumped parameter, thermal network code; thus the general layout shown in Figure 10 must be broken down into a "node and arc" directed graph structure. This simple model of the recuperative MR can be viewed as four coupled heat exchangers: two at the hot and cold boundaries describing heat transfer between the working fluid and the outside world and two describing heat transfer between the working fluid and magnetic material in the recuperative wheel sections.

As mentioned above, three stages were used to span the $20 \mathrm{~K}$ to $80 \mathrm{~K}$ range in the model. For comparison with the other concepts, the load on the lowest stage was taken as the latent heat of hydrogen plus one-third of the sensible heat and ortho-para conversion energy that must be removed from the hydrogen as it was cooled from 80 $\mathrm{K}$ to $20 \mathrm{~K}$. The load on the second stage was the heat rejected from the first stage plus one-third of the sensible heat and ortho-para conversion energy of the hydrogen. The load on the upper stage was the heat rejected from the second stage plus the remaining third of the sensible heat and ortho-para conversion energy of the hydrogen. All three stages used a peak magnetic field of 7 Tesla and had a wheel diameter of $0.76 \mathrm{~m}$. The rotation rate was $12 \mathrm{rpm}$ or $0.2 \mathrm{~Hz}$. The helium pressure in the two lower stages was $12 \mathrm{~atm}$-in the upper stage a helium pressure of $20 \mathrm{~atm}$ was used.

This model of 0.1 ton/day liquefier has a liquefaction efficiency of $29.4 \%$. The model is not optimized for efficiency-it is overdesigned, to some extent, so with further work the efficiency could be raised by several percent-but it gives some idea of the high liquefaction efficiencies that can be obtained from a recuperative device. The main problem with this device lies in the heat transfer geometry. To obtain the heat transfer area per volume needed in the lower stage, the plate thickness is 0.048 $\mathrm{mm}$ with a space between the plates of $0.40 \mathrm{~mm}$. For a plate length of $8.1 \mathrm{~cm}$, this requires 200 plates. In our opinion, this geometry is difficult to fabricate, particularly because the structure must also tolerate the high magnetic stresses in the magnetization and demagnetization processes.

It is possible to increase the plate thickness, but this occurs at the expense of efficiency. If the thickness is increased (i.e. the cross-sectional area becomes larger) an imbalance in the total heat capacities of the magnetic material and working fluid in the recuperative heat exchanging sections is created. This imbalance severely 
decreases the efficiency, but it can be compensated for by either slowing the wheel rotation rate or increasing the mass flow rate of the working fluid. However, decreasing the wheel speed will adversely affect the heat transfer coefficient and increasing the flow rate will lead to higher pumping losses: both alternatives lead to decreased efficiency.

These considerations effectively eliminate the parallel plate geometry. Turning to packed beds, we can compute the porosity (the ratio of fluid flow area to the total cross-sectional area) from the heat transfer geometry parameters. The calculated value is between $85 \%$ and $90 \%$, which is much too high. To bring the porosity down to a more practical level requires increasing the amount of magnetic material presentwhich again causes an imbalance in the recuperative sections of the wheel, and leads to the same problem that we have in the parallel plate case. Also, as noted previously, the flow control problem for the packed bed geometry is extremely challenging; thus the packed bed concept is also not practical.

To summarize, the 0.1 ton/day recuperative magnetic liquefier has a reasonably good efficiency theoretically; however, the heat transfer geometry required for the maximum efficiency is difficult to fabricate with current technology.

\section{The Active Magnetic Regenerative Refrigerator (AMR) Model}

The schematic of the Active Magnetic Regenerator (AMR) in Figure 11 is shown as a packed particle bed of magnetic material. The regenerator bed is sandwiched between the Hot and Cold Heat Exchangers (HHEX and CHEX). The heat transfer fluid is typically helium in the cryogenic regime, though hydrogen is a possibility in a hydrogen liquefier. The pistons shuttle the fluid between the reservoirs.

A complete cycle of the AMR is as follows: with the shuttle fluid all in the cold reservoir, the magnetic material in the bed is adiabatically magnetized. The bed warms by the magnetocaloric effect. The fluid is then passed from the cold to the hot reservoir. With the fluid now in the hot reservoir, the bed is adiabatically demagnetized. The bed cools by the magnetocaloric effect. The fluid is then passed from the hot back to the cold reservoir.

Figure 12 illustrates temperature profiles of the bed and fluid as a function of bed

position. For simplicity, the adiabatic temperature change of the material is assumed to be independent of temperature. 
Reciprocating

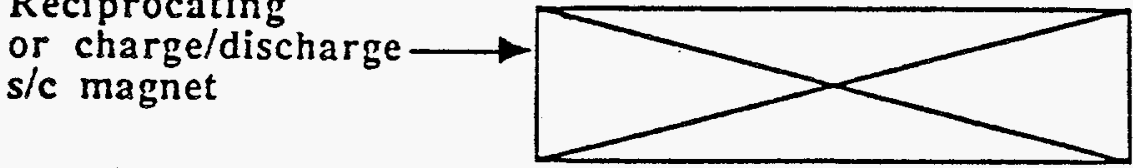

Heat exchange fluid reservoir
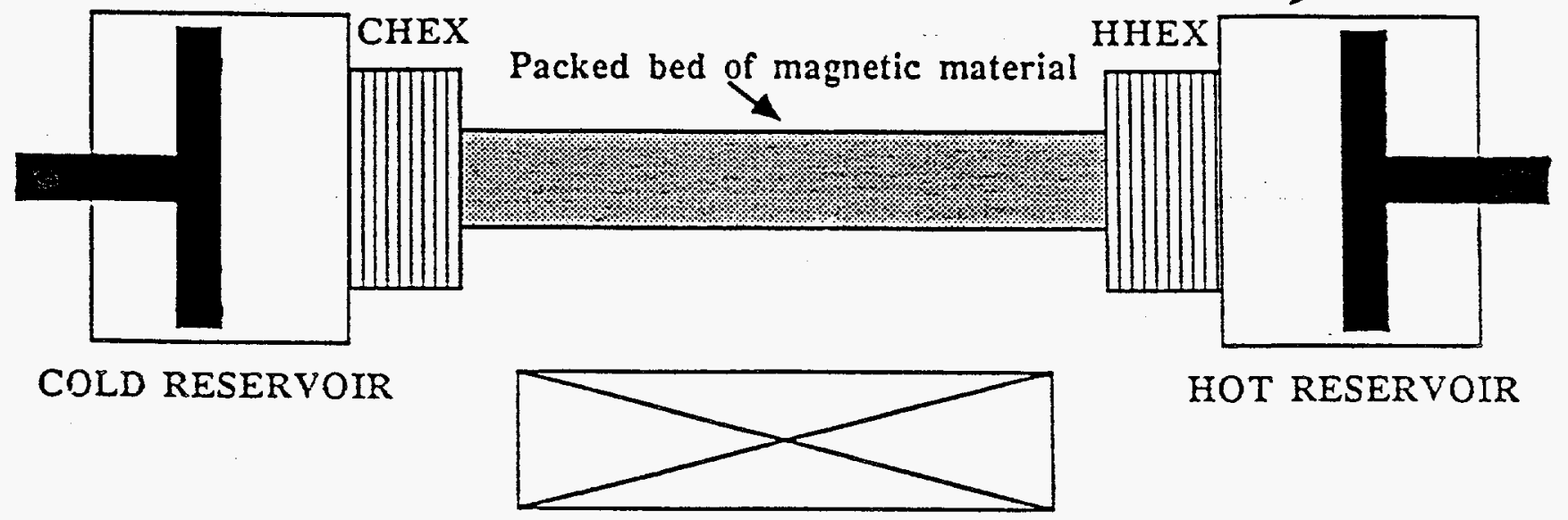

HOT RESERVOIR

Figure 5.2.1-1. Schematic of the AMR.

Figure 11. Schematic of the Active Magnetic Regenerator 


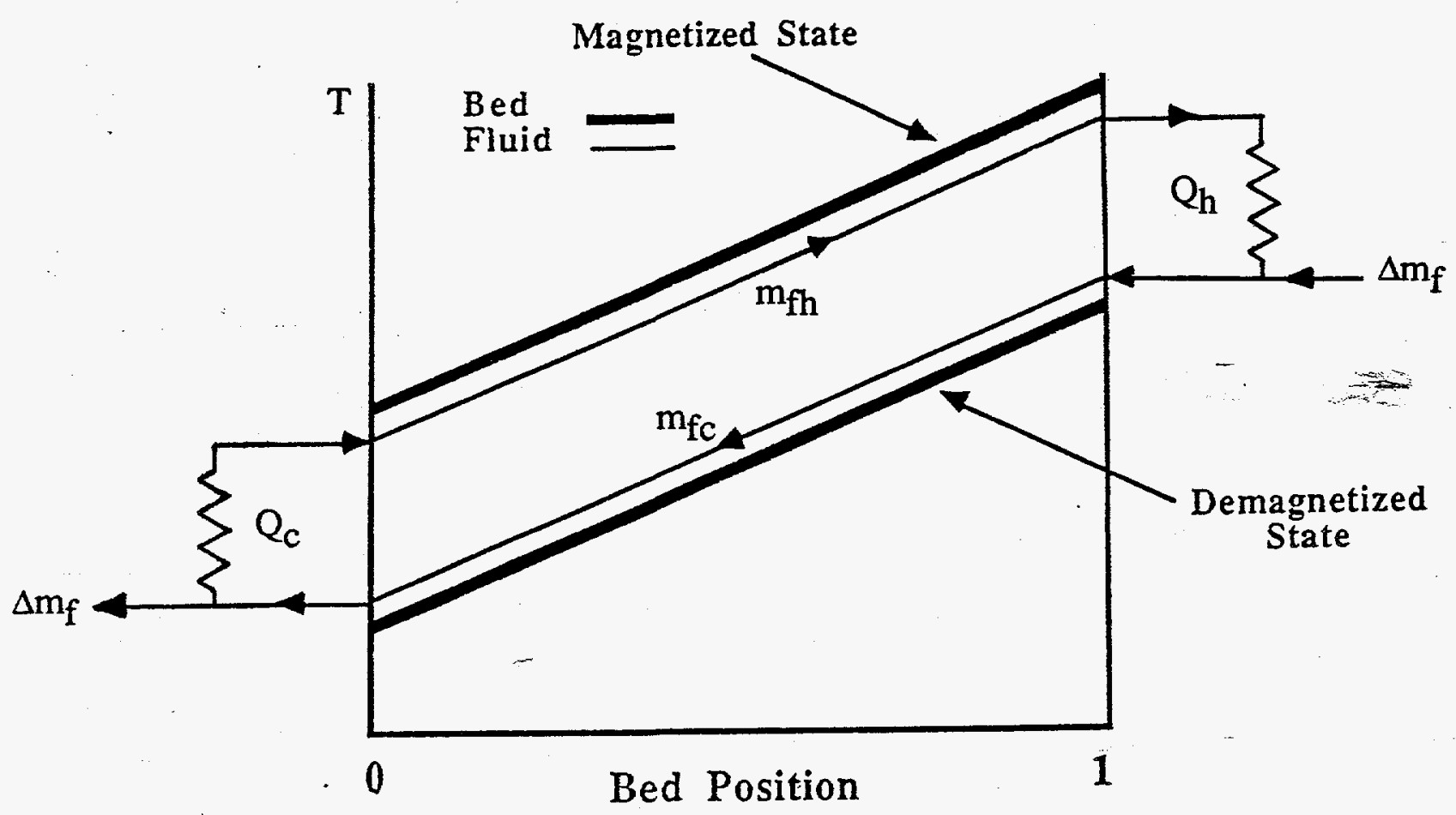

Figure 12. Temperature Profiles for AMR Bed/Fluid in the Infinite Bed Thermal Mass Limit 
The general case is illustrated where the total mass of fluid flowing in the cold direction, $\mathrm{m}_{f c}$ may be different from that flowing in the hot direction, $\mathrm{m}_{f h}$. For simplicity, the bed heat capacity is assumed to be much larger than the shuttle fluid, because the temperature profiles do not change over the flow periods in that limit. The upper profiles correspond to the flow from the cold to the hot reservoir while the bed is magnetized. The fluid enters the bed at the temperature of the cold reservoir $T_{c}$ because it has just left the cold heat exchanger. Because the bed temperature rises going from the cold to the hot end, the fluid temperature rises as it exchanges heat with the bed and leaves the bed at a temperature greater than the hot reservoir $\mathrm{T}_{h}$. Passing through the hot heat exchanger, the fluid temperature drops to $\mathrm{T}_{h}$, giving up an amount of heat

$$
Q_{h}=m_{f h} c_{p} \Delta T_{h}
$$

After the flow is complete, the bed is adiabatically demagnetized, achieving the lower profile shown in Figure 12. The flow proceeds then from the hot to the cold end. Upon entering the bed, the fluid temperature is $T_{h}$. Exchanging heat with the bed, it drops to a value below $\mathrm{T}_{c}$ at the cold end. Going through the cold heat exchanger, it absorbs heat equivalent to

$$
Q_{c}=m_{f h} c_{p} \Delta T_{c}
$$

The enthalpy change for the fluid of mass $\Delta m_{f}=\dot{\mathrm{m}}_{f c}-\dot{\mathrm{m}}_{f h}$ in going from temperature $\mathrm{T}_{h}$ to temperature $\mathrm{T}_{c}-\Delta \mathrm{T}_{c}$ is

$$
\Delta H=\left(m_{f c}-m_{f h}\right)\left(h\left(T_{c}-\Delta T_{c}\right)-h\left(T_{h}\right)\right)
$$


The work input over the cycle is given by the first law for an open system:

$$
W=Q_{h}-Q_{c}+\Delta H
$$
given by

The ideal or minimum work required to cool the fluid and provide cooling $\dot{Q}_{c}$ is

$$
W_{\text {ideal }}=\left(T_{h} \Delta S-\Delta H\right)+Q_{c} \frac{T_{h}-T_{c}}{T_{c}}
$$

The efficiency can be defined as

$$
\eta=\frac{W_{\text {ideal }}}{W}
$$

The AMR is unique among regenerative or recuperative magnetic or gas cycles in that an element of working magnetic material only goes through a simple nonregenerative Brayton cycle about a specific temperature determined by its location in the bed. The heat transfer fluid links these cycles together to span a large temperature range. The AMR has a significant advantage because any given element of magnetic material does not have to be active over the entire temperature range of the device.

In analyzing the AMR, we took the approach of first writing the general equations and attempting to solve them. We then implemented approximations as necessary to obtain a useful design tool model.

A model which solves the complete physical problem in one-dimension for the AMR was developed by Barclay. ${ }^{12}$ The computer code MRRAP integrates the full 
one-dimensional time-dependent equations using a finite difference scheme.

To conduct our design studies, it is necessary to make simplifications in these equations. If we assume the pore fluid heat capacity is negligible, we have the following equations ${ }^{4}$ relating the bed temperature $t_{b}$ and fluid temperature $t_{f}$ as a function of position $\mathrm{x}$ and time t:

$$
\begin{gathered}
h A\left(t_{b}-t_{f}\right)=\dot{m}_{f} c_{p} L \frac{\partial t_{f}}{\partial x} \\
h A\left(t_{f}-t_{b}\right)=M_{b} c_{b} \frac{\partial t_{b}}{\partial t}
\end{gathered}
$$

where $h$ is the heat transfer coefficient between the fluid and solids in the bed; $A$ is the total cross-sectional area between the fluid and solids; $L$ is the bed length; $M_{b}$ and $\mathrm{m}_{f}$ are the bed mass and fluid flow rate; and $c_{b}$ and $c_{p}$ are heat capacities of the bed and fluid.

Equations 7 and 8 can be cast into dimensionless form by a reduced length $\Lambda$ and reduced period $\Pi$. where

$$
\begin{aligned}
& \Lambda=\frac{h A}{\dot{m}_{f} c_{p}} \\
& \Pi=\frac{P h A}{M_{b} c_{b}}
\end{aligned}
$$

$P$ is the time period of the flow in either direction.

We have solved equations 7 and 8 numerically. This version of the model is computationally intensive. We have, therefore, developed a model in which the bed mass is assumed to be infinite, corresponding to zero reduced period. This assumption eliminates the time dependence in the problem allowing us to obtain solutions very rapidly. The limit is useful since it represents the point of maximum refrigerator efficiency. It is also a physically realizable limit to a good approximation. 
The equations in the infinite bed mass limit are

$$
\begin{gathered}
\frac{\partial t_{f h}}{\partial x}=\Lambda_{h}\left(t_{b h}-t_{f h}\right) \\
\frac{\partial t_{f c}}{\partial x}=\Lambda_{c}\left(t_{f c}-t_{b c}\right) \\
t_{b h}=t_{b c}+\Delta t_{a d} \\
h_{h} A P_{h} \frac{\left(t_{f h}-t_{b h}\right)}{t_{b h}}=h_{c} A P_{c} \frac{\left(t_{f c}-t_{b c}\right)}{t_{b c}}
\end{gathered}
$$

The subscripts $c$ and $h$ refer to the cold blow (flow from the hot to cold reservoirs) and the hot blow (flow from the cold to the hot reservoirs), respectively; $\Delta \mathrm{t}_{a d}$ is the adiabatic temperature change of the magnetic material. Equation 13 is the algebraic equation which results from the two time-dependent equations (see equation (8)) when we go to the infinite bed mass limit.

Equations $10-13$ are solved numerically by a shooting method. ${ }^{5}$ We integrate from the cold end knowing that the hot fluid stream is at temperature $t_{c o l d}$ and guessing at the temperature of the cold fluid stream. Integrating across the bed, we obtain a value for the cold fluid stream at the outlet which we want to be $t_{h o t}$. We repeat the integrations, adjusting our guess for the temperature of the cold fluid stream at the cold end until we obtain the matching value at the hot end to some desired degree of accuracy.

Both versions of AMR models have been checked against each other in the appropriated limit, and also against known results for passive regenerators. ${ }^{4}$

Various geometries are possible for the bed. These include: packed particles, closely spaced plates parallel to the direction of flow, perforated plates or screens perpendicular to the direction of flow, a solid block with fine channels, and so on. We consider packed particle beds here because they are simple and inexpensive to construct. 
The heat transfer coefficient between fluid and bed is obtained from empirical correlations for packed particle beds. ${ }^{6}$ Variations in properties with temperature of both helium $^{7}$ and the magnetic material are accounted for. Pumping losses, thermal conduction losses across the bed, and axial dispersion are taken into account separately using well-established empirical correlations from the literature. $8,9,10$

AMR performance is sensitive to the thermodynamic properties of the magnetic material. The following argument gives the "ideal" material properties for an AMR in the general case where the flow can be unbalanced: Substituting equations 10 and 11 into equation 13 , we get

$$
\dot{m}_{f h} P_{h} \frac{1}{t_{b h}} \frac{\partial t_{f h}}{\partial x}=\dot{m}_{f c} P_{c} \frac{1}{t_{b c}} \frac{\partial t_{f c}}{\partial x}
$$

For peak efficiency, entropy generation should be minimized. Heat transfer is between bed and fluid. Entropy generation will be minimized if

$$
\begin{aligned}
& t_{f h}=t_{b h} \\
& t_{f c}=t_{b c}
\end{aligned}
$$

Substituting 15 and 16 into 14 and integrating, we get,

$$
\begin{gathered}
\frac{\partial}{\partial x}\left(\log t_{b h}-\frac{m_{f c}}{m_{f h}} \log t_{b c}\right)=0 \\
t_{b h}=c t_{b c}^{\frac{m_{f c}}{m_{f h}}}
\end{gathered}
$$

where $\mathrm{c}$ is a constant. 
Combining this with equation 12 , we get

$$
\Delta t_{a d}=f(\Delta H) t_{b c}^{\frac{m_{f c}}{m_{f h}}}-t_{b c}
$$

For balanced flow, the adiabatic temperature change is linearly proportional to the temperature, with the intercept at 0 .

Figure 13 shows computed temperature profiles over a complete AMR cycle. In this sample problem, the hot and cold fluid flow periods and the field change period are all $1.0 \mathrm{~s}$. The helium gas flow is $0.53 \mathrm{~g} / \mathrm{s}$ at an average pressure of $1.0 \mathrm{MPa}$. The bed length and cross section are $5 \mathrm{~cm}$ and $2.84 \mathrm{~cm}^{2}$, respectively. The particle size is $0.015 \mathrm{~cm}$. The magnetic material is $\mathrm{GdNi}_{2}$ and the field change is $7 \mathrm{~T}$. 

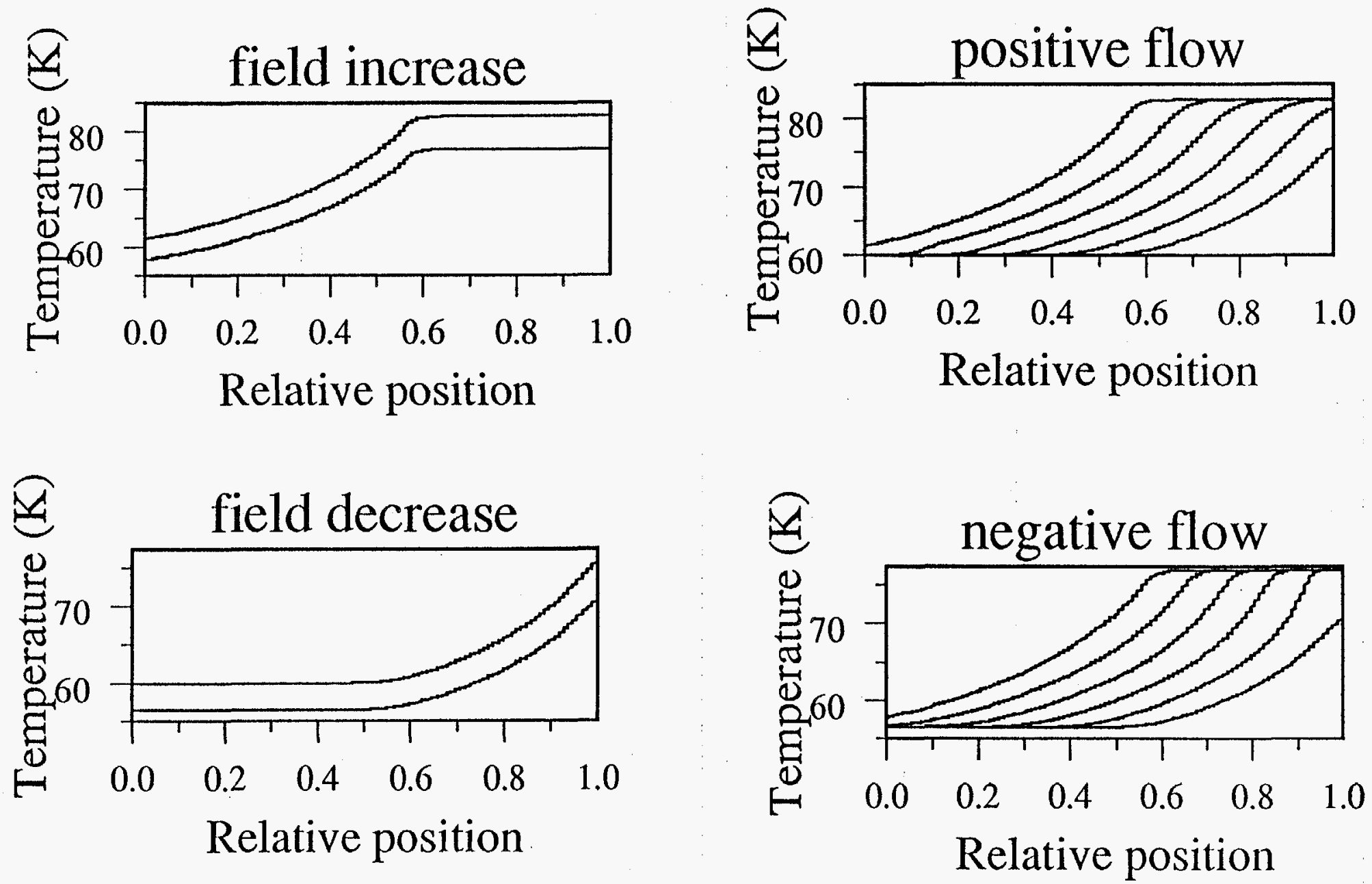

Figure 13. Bed Temperature Profiles over an AMR Cycle 


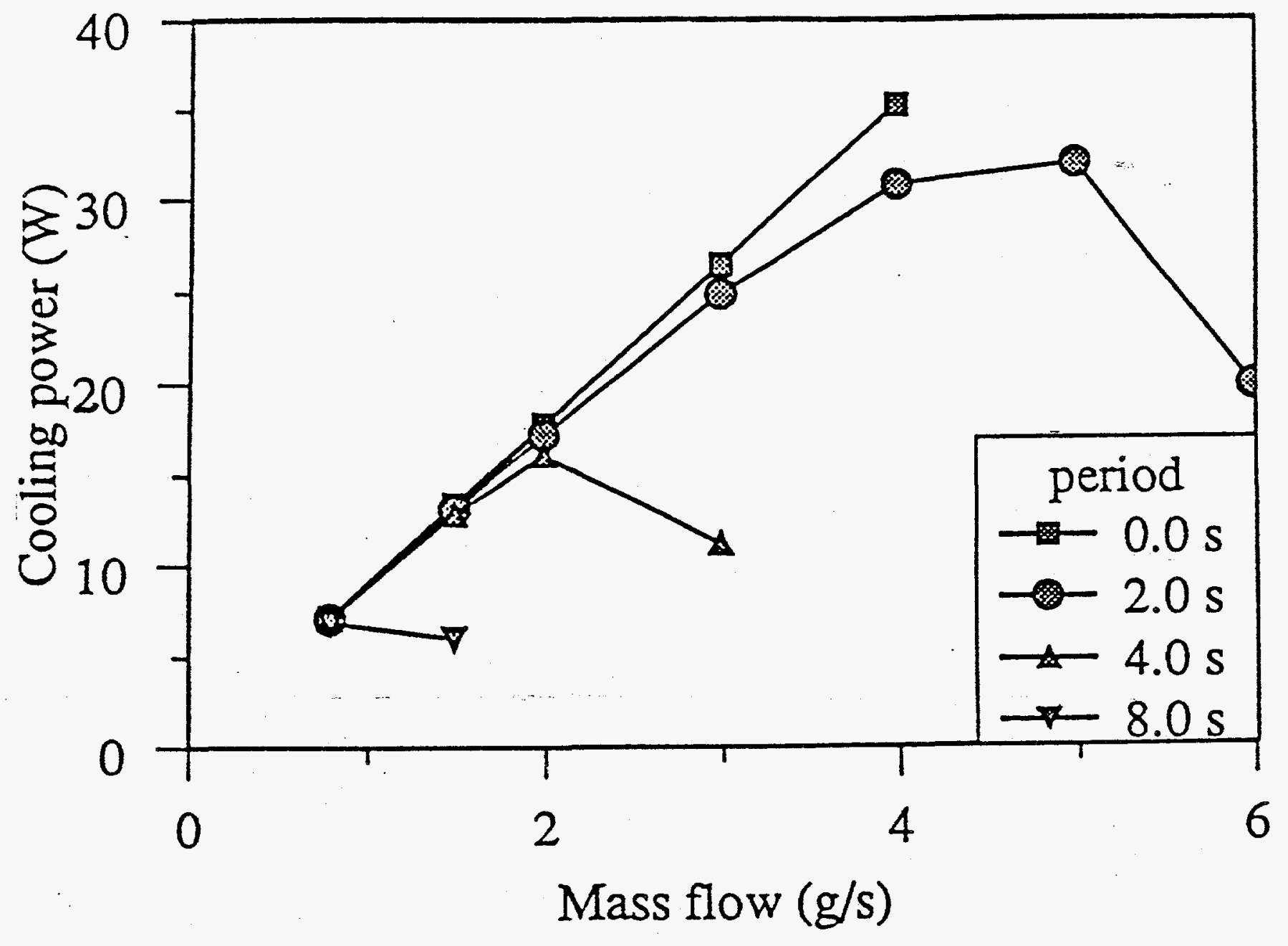

Figure 14. Load Curves at $7 \mathrm{~T}$ for $\mathrm{GdNi}_{2}$ 
Temperature profiles over the four parts of the AMR cycle are shown in the figure: Over the field increase (decrease), the lowest (highest) profile shows the bed temperature before the field increase (decrease), and the highest (lowest) profile shows the bed temperature after the field increase (decrease). Over the positive and negative flow periods, profiles are shown at equal time intervals. The uppermost (lower-most) profile shows the bed at the start of the positive (negative) flow. Lower (upper) profiles in succession show successively later time intervals with the lowest (highest) profile representing the bed at the end of the positive (negative) flow period.

Figure 14 shows computed load curves for several values of the total period. The AMR parameters are the same as those described for Figure 13, except for the variation in period and fluid flow. Note that points represent the actual values. The connecting lines are there for continuity.

The results shown for zero period are from the time-independent, zero-reduced period computations described above. The time-dependent computations show consistency with this limiting result.

For the non-zero period, the cooling power curves turn over at particular values of mass flow. This is because the heat capacity of the fluid shuttling through the bed becomes greater than the heat capacity of the bed material. The bed can no longer "regenerate" the fluid. The zero period curve would also turn over at high enough mass flow, but for a different reason. At high enough mass flow, the heat transfer between the fluid and bed becomes poor. The model predicted high efficiencies under reasonable operating conditions with no fundamental manufacturing limitations.

Therefore, based on the analyses of the conceptual designs considered, and following a conceptual design review with external reviews from DOE and Argonne National Lab, the AMR cycle was selected for further development in the preliminary design tasks of Phase I. This selection reflects the strengths of the AMR cycle in the liquefier application. This cycle benefits from superior efficiency and compact packaging over the other concepts considered.

\section{Subscale Prototype Preliminary Design}

This chapter reviews the preliminary liquefier designs, based on the AMR cycle selected in Chapter 3. Preliminary specifications are highlighted, followed by detailed discussions of AMR test and modeling experience. This testing both validates the AMR cycle and legitimizes it as an appropriate choice for liquefier application. Preliminary designs are discussed last, with an emphasis on overall configuration.

Subscale liquefier performance requirements established a baseline set of specifications, such as cooling power, efficiency, and temperature span, for 
comparative evaluation of the alternative magnetic refrigerator concepts. The AMR cycle was chosen among four candidate magnetic refrigerator designs evaluated in the Conceptual Design Phase. These included recuperative, external regenerative, internal regenerative, and active magnetic regenerative cycles. The selection of the AMR cycle for the liquefier application provides a basis for further refinement of these specifications for the preliminary design. These specifications are both quantitative and qualitative, reflecting the level of detail carried through the conceptual design phase. They are delineated below.

- The refrigerator must generate sufficient cooling to produce the equivalent of approximately 0.1 ton/day of liquid parahydrogen at 1 atm and $20 \mathrm{~K}$ from 40 atm hydrogen feedstock at room temperature. This liquefaction rate is equivalent to $50 \mathrm{l} / \mathrm{hr}$ or $1.05 \mathrm{~g} / \mathrm{s}$. In the test mode of operation, the actual ortho-to-para conversion can be incomplete or may not occur at all in the absence of catalysts, so long as the heat of conversion from this exothermic reaction is taken into account.

- The 40 atm hydrogen feedstock gas will be precooled to approximately $77 \mathrm{~K}$ by heat exchange with gaseous and liquid nitrogen.

- The refrigerator will operate on the AMR cycle using 20 atm gaseous helium as the heat transfer fluid between the magnetic material and the hydrogen feedstock.

- The AMR cycle will use either two or three stages to operate from $77 \mathrm{~K}$ to $20 \mathrm{~K}$ because no single magnetic refrigerant can span the entire range.

Several preliminary AMR liquefier designs meeting the above specifications were evaluated in the process to select the most promising configuration to be carried forward in the detailed design tasks of Phase I. These configurations fall into two distinct categories. The designs in the first category use a rotary configuration. They all generate continuous cooling by coupling many small AMR cycles in series, with each at any given time, at a different point in its cycle. The designs in the second category use a linear reciprocating configuration. These generate intermittent cooling over a fixed AMR cycle period.

These preliminary AMR liquefier designs draw upon the extensive experience gained from the development and testing of the Astronautics Proof-of-Principle AMR refrigerator. The following sections detail these activities to establish a firm grasp of the current state of the art for the reader. This provides a basis for the discussions of the preliminary designs in the last sections of this chapter.

In Section 4.1, the Active Magnetic Regeneration Principle will be presented. A description of the experimental apparatus and the testing procedure will be given. Section 4.2 will present a detailed summary of the test results achieved using $\mathrm{Er}_{0.86} \mathrm{Gd}_{0.14} \mathrm{Al}_{2}$ and $\mathrm{GdNi}_{2}$ in the AMR apparatus. The test results will be compared to modeled data from a time-dependent AMR model. A summary of the results along with some conclusions will be presented in Section 4.3. 


\subsection{Proof-of-Principle Active Magnetic Regenerative Refrigerator}

\subsubsection{AMR Principle}

The AMR concept was tested with an experimental refrigerator designed to operate within the temperature range of about $4 \mathrm{~K}$ to $80 \mathrm{~K} .{ }^{11}$ The device uses a single reciprocating superconducting solenoidal magnet in persistent mode to produce a uniform field over the two in-line beds of magnetic material. No fluid flow occurs during field change.

There are two modes in which the AMR is shown to work well. ${ }^{12,13}$ In one mode, the heat capacity of the fluid in the bed pore volume is large compared to the magnetic material. The magnetic material follows a regenerative cycle in which the fluid in the bed acts as the regenerator. In the other mode, the pore fluid heat capacity is negligible compared to that of the magnetic material. The elements of magnetic material follow either non-regenerative Brayton or Carnot cycles connected in parallel by the heat transfer fluid. The former mode applies to devices that use either liquid or high pressure helium. The latter mode applies to the AMR device used in this program.

It has been shown that ${ }^{14}$ for an AMR in which the bed goes from a uniform high to zero field, an 'ideal' magnetic material exists in which the adiabatic temperature change is linearly proportional to the absolute temperature. Ferromagnetic materials below their Curie point exhibit this approximate behavior. This allows for the choice of a wide range of materials and temperatures for AMR operation.

Between magnet motions, a double-acting piston displacer moves heat transfer fluid (helium gas) through the beds. The fluid absorbs heat at the CHEX and rejects heat at the HHEX.

\subsubsection{Experimental Apparatus}

Key components of the device are illustrated in Figure 15. Two AMR beds are connected to a common CHEX. The beds are connected to separate HHEXs, at opposite ends of an aluminum thermal bus. One end of the thermal bus is connected to the bottom of a Gifford McMahon (GM) refrigerator. A reciprocating magnet alternately magnetizes one bed while demagnetizing the other. When the magnet is stationary, a double-acting piston displacer forces gas through one of the HHEXs, through one of the beds, through the CHEX, through the other bed, through the other HHEX, and back to the displacer. A heater is attached to the CHEX to apply a load to the AMR. 
Figure 16 shows the complete device to scale. The NbTi solenoid magnet is contained in an annular, stainless steel helium dewar. A soft iron flux-return moves with the magnet and reduces the stray field which would otherwise produce eddy current heating in the dewar and ball bearing wheels which guide the magnet on tracks. The field outside the iron at the mid-plane is below $0.1 \mathrm{~T}$. Separate linear actuators, at room temperature, drive the magnet and displacer. Two position control systems independently move the magnet and displacer piston.

The displacer uses two circular spring-loaded ultra high density polyethylene seals mounted on the piston and sliding on a polished cylinder wall, to eliminate leakage.

The HHEXs are made by cutting narrow closely-spaced grooves in a cylindrical aluminum bus in the axial direction then placing sleeves over them. By the appropriate plumbing, helium gas is forced through the grooves to effect heat exchange.

The CHEX presents unique problems. Large, high conductivity loops must be avoided to prevent eddy-current heating from the changing magnetic field. Also, a large thermal mass is desirable at operating temperatures below $10 \mathrm{~K}$, to avoid large thermal fluctuations. To address both issues, a tube-in-shell heat exchanger was devised which uses fluid helium for heat capacity.

The GM refrigerator used in the tests is a Cryomech GB04. The apparatus is designed to accept the more powerful Cryomech GB37 also.

Accurate measurement of temperature, load power, pressure difference, and displacement are the primary requirements for performance testing of the AMR refrigerator. The device is instrumented with more than 30 temperature sensors. These include thick-film Platinum Resistance Thermometers (PRTs) from Omega Engineering, Carbon Glass Resistors (CGRs) from Lakeshore Cryotronics, ${ }^{15}$ and Bismuth Ruthenium Oxide (BRO) resistance thermometers ${ }^{16}$ developed in-house. Calibrations for the sensors were standard Deutsche Industrial Norms (DIN) curves for the PRTs, manufacturer-provided for the CGRs, and produced in-house for the BROs. For the critical lower temperature readings (below $40 \mathrm{~K}$ ), accuracy of $+/-0.2 \mathrm{~K}$ is adequate. For higher temperatures, errors of up to $+/-1.5 \mathrm{~K}$ were deemed tolerable. 


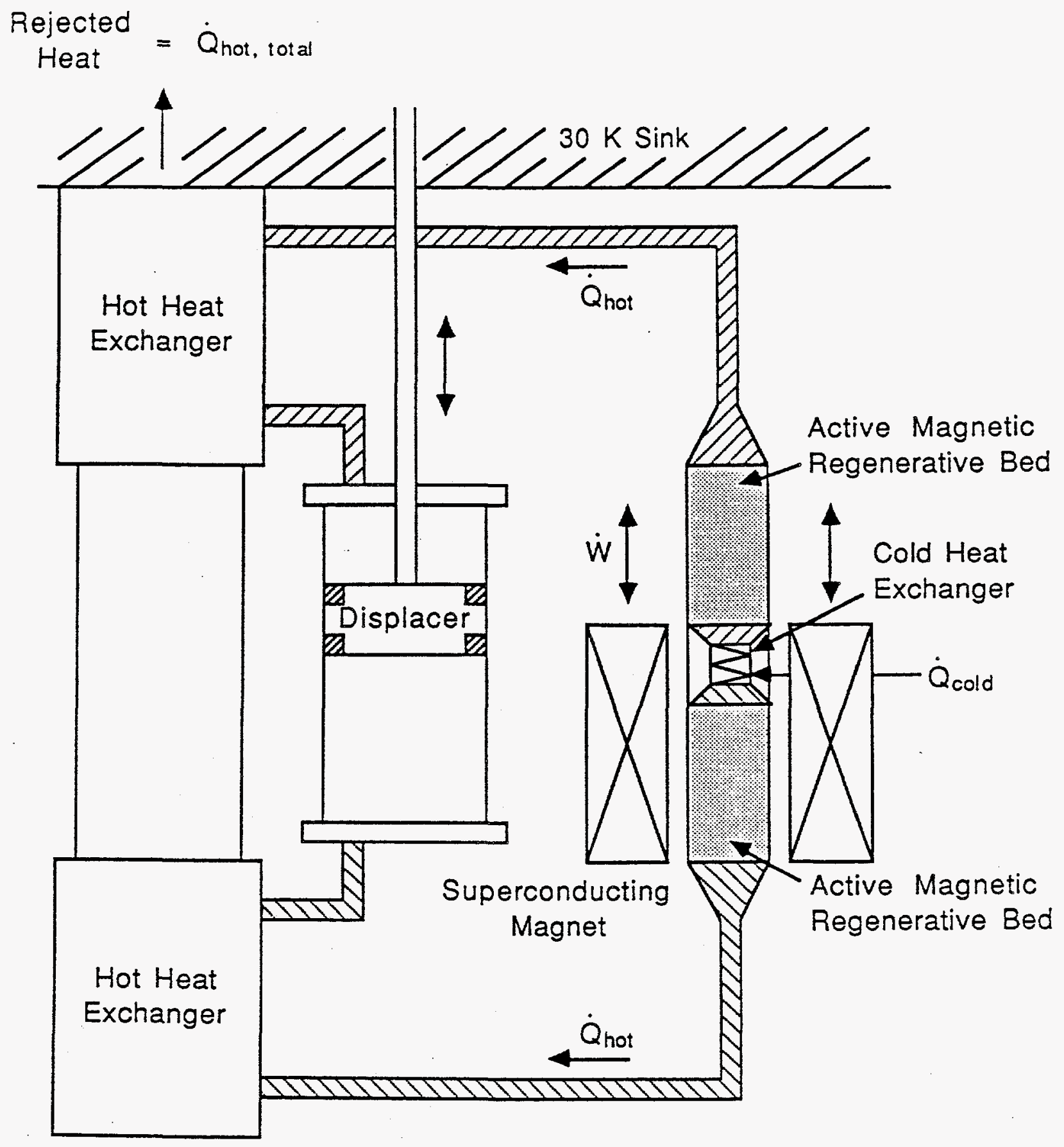

Figure 15. Key Components of the AMR Experiment 


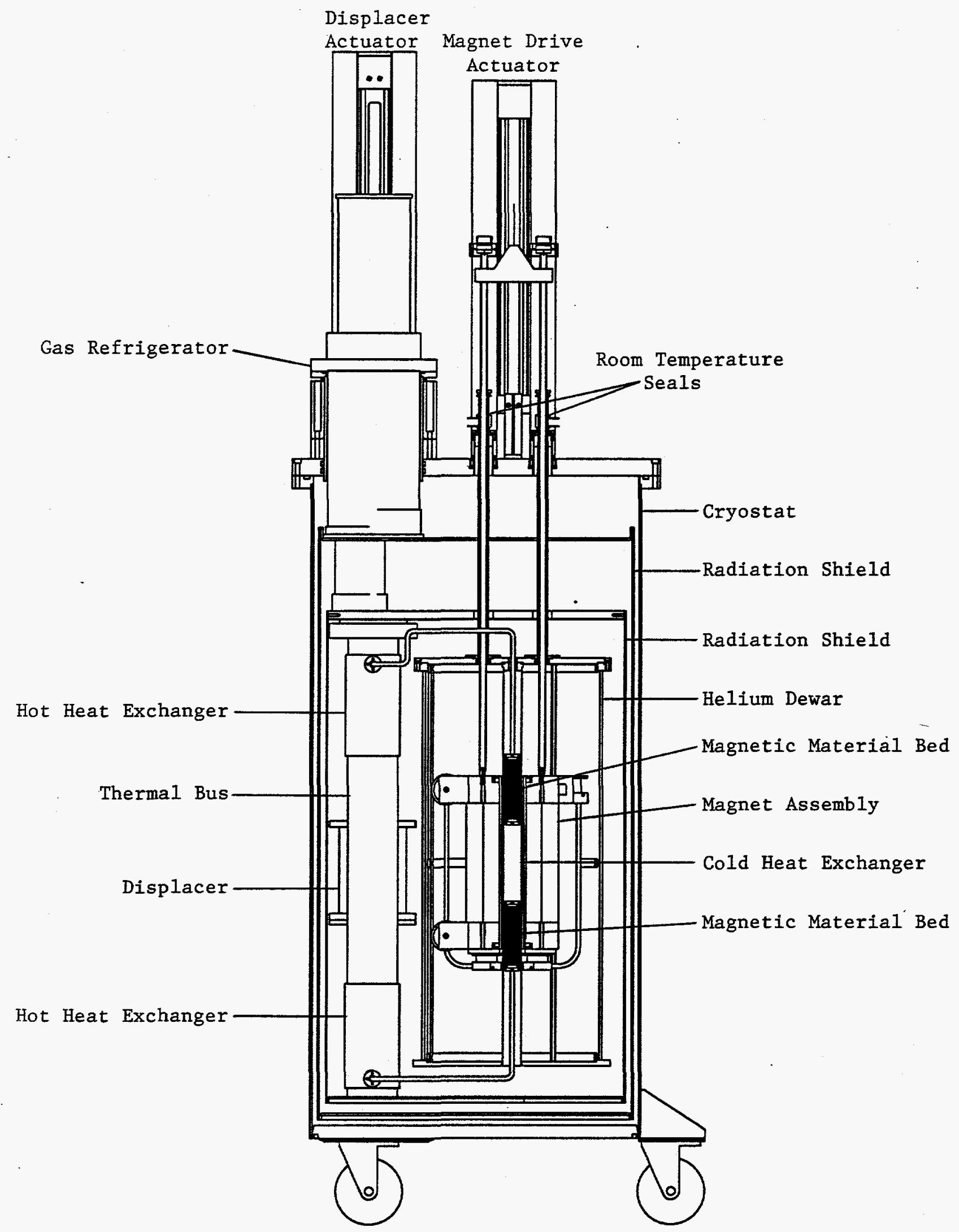

Figure 16. Scale Drawing of the AMR Proof-of-Principle Experiment 
The most critical temperature measurements center on the CHEX, HHEX, and the magnetic material beds. The heat exchanger temperatures are used as the cold and hot sink temperatures of the refrigeration cycle. The CHEX is fitted with a CGR and $\mathrm{BRO}$, both relatively insensitive to magnetic fields. The HHEX, consisting of two finned regions, is fitted with PRTs and BROs on both finned sections. Measurement of the temperature at different points along the regenerator beds is desirable to show the effects of magnetization and gas flow. A narrow, streamlined sensor consisting of five BROs linearly spaced at $2 \mathrm{~cm}$ intervals is epoxied along the inside wall of each regenerator bed during assembly for tests at temperatures less than $40 \mathrm{~K}$. These sensors are in contact with the helium heat transfer fluid in the beds.

Displacement and pressure difference measurements are also critical. The displacement of the reciprocating magnet is measured using a built-in linear potentiometer within the linear actuator located at room temperature. Displacer motion is similarly measured. To understand the flow of gas out of the displacer and to check on blow-by past the seals on the displacer piston, a diaphragm pressure sensor (calibrated in liquid helium for $\mathrm{Er}_{0.86} \mathrm{Gd}_{0.14} \mathrm{Al}_{2}$ and in liquid nitrogen for $\mathrm{GdNi}_{2}$ ) is placed across the displacer. Experimental observations do not indicate the occurrence of blow-by.

The apparatus, as originally constructed, had displacer actuator performance adequate for $\mathrm{Er}_{0.86} \mathrm{Gd}_{0.14} \mathrm{Al}_{2}$ testing but proved to be inadequate for $\mathrm{GdNi}_{2}$ testing. The higher temperature of operation resulted in great friction, causing the actuator motor to overheat. It was also desired to operate with higher mass flow rate and increase frequency in the future. New power electronics were obtained for both actuators, a new actuator was obtained for the displacer, apparatus modifications to accommodate the new actuator were made, and the computer control software was rewritten. These changes improved performance significantly, permitting higher mass flow rates and better control of refrigeration cycle execution. Internal protection to prevent motor overload due to sudden changes in motion, internal control to permit rapid directionof-motion changes, and better location sensing are some of the new functions added via the hardware/software modifications.

\subsubsection{Testing Procedure}

A Cryomech GB04 GM refrigerator pre-cools the AMR refrigerator and also serves as its hot sink. The magnet dewar cools slowly because of its thermal isolation to minimize helium boil-off during operation. Cooling is facilitated by flushing the magnet dewar with liquid nitrogen. The dewar (25 liter free volume) is then filled with liquid helium and the magnet is charged and switched into persistent mode. The current leads, which screw into the top of the magnet, are removed to reduce heat leak and allow the magnet to move more freely. The system typically takes 2-3 days to cool down. 
An IBM-AT compatible computer is used to control the linear actuators which drive the magnet and displacer. Velocity profiles of trapezoidal and modified square position wave forms are typically used with various periods and amplitudes.

An HP 3852A data logger is used to measure and store the data from the experimental sensors. It communicates sensor readings and results to an HP 310 computer. Table 2 includes a list of all the sensors used in the data acquisition.

The HP 310 computer controls the data logger. It sets up the measurement, programs the data logger, and receives results from the logger. The computer provides real time plotting and stores data on disk for later retrieval. The plotting feature enables plotting of key variables while the experiment is in progress. This feature was needed to determine trends and decide when a response to changes in the input variables has completed, or steady state has been achieved, before applying new input variables.

To accurately measure many channels simultaneously is one of the main advantages of using this HP data acquisition system. The HPIB (IEEE 488) interface bus makes it very easy to interface with many channels simultaneously. The HP 310 also uses the BASIC language which makes programming the data acquisition system simple. However, the more channels data is collected from, the slower the data acquisition rate. So the HP data acquisition speed capability is limited by the number of channels used.

A run starts by inputting the displacer motion to obtain the desired mass flow rate of helium gas through the beds. The period of the magnet motion is then adjusted to synchronize the flow of gas through the beds with magnetization of the beds. Data is sometimes taken quickly to capture transients and sometimes more slowly to capture steady-state conditions. Typically, no-load tests are done first at a particular field. Loads are then applied to the CHEX in small power intervals until the HHEX temperature reaches the desired temperature of operation. 
Table 2. List of All Sensors Used in the Data Acquisition

Sensor Location

1st stage bed 1 warm term lst stage bed 1 cold terp 1st stage bed 2 warm temp 1st stage bed 2 cold term 2nd stage bed 3 warm tant 2nd stage bed 3 cold termp 2nd stage bed 4 cold tamp He $0 / P \# 1$ inlet temp
He $0 / P \# 2$ outlet temes He purm inlet temp He pump outlet temp He LN2 EEX inlet temp He LN2 HEX outlet temp B2 LN2 HEX inlet temp H2 LN2 BEX outlet temp B2 $O / P$ \#6 inlet tentp H2 O/P \#5 temp $\mathrm{H} 2 \mathrm{O} / \mathrm{P} \# 4$ temp H2 O/P \#3 temp $\mathrm{H} 2 \mathrm{O} \mathrm{P} \# 2$ temp E2 O/P \#1 outlat temp LH2 stoxage tank temp Magnet demountable lead terp Magnet demountable lead temp Magnet 11 temp

Persistant mode switch temp Persistant mode awitch ter 1st stage bed 1 post tenp 2nd stage bed 3 post temp 2nd stage bed 4 post temp Radiation shield over beds 1 . 2 tento Radiation shield over beds 384 tom Radiation shield under beds 182 temp Radiation shield under beds $3 \& 4$ temp Ist stage bed I backup warm termp 1st stage bed 1 backup cold term 1st stage bed 2 backup warm temp 1st stage bed 2 backup cold term 2nd stage bed 3 backup warm term 2nd stage bed 3 backup cold term 2nd stage bed 4 backup warm termp 2nd stage bed 4 backup cold 1st stage bed 1 diff press temp 1 st stage bed 2 diff press tant 2nd stage bed 3 diff press temp 2nd stage bed 4 diff prese te He $0 / P$ HEX diff press Ho LN2 HEX dife press tern H2 IN2 HEX diff pross temp Three way valve A1 press temp
Three way valve A2 press temp
Type

Platinum Resistor

Bismuth Ruthenium Oxide Resistor Rlatinum Resistor

Oxide Resistor Bisteristor Bismuth Ruthenium Oxide Resistor Bismuth Ruthenium Oxide Resistor Silicon Diode Silicon Diode

Platinum Resistor

Platinum Resistor

Platinum Resistor

Platinum Resistor

Platinum Resistox

Silicon Diode

Sillicon Diode

Silicon Diode

Silicon Diode

Siliticon Diode

Silicon Diode

Silicon Diode

TRD

Silicon Diode

Silicon Diode

IBD

Platinum Resistor

Platinum Resistor

Platinum Resistor

Platinum Resistor

Platinum Resistor

Platinum Resistor

Platinum Resistor

Platinum Resistor

Bismuth Ruthenium Oxide Resistor

platinum Resistor

Blsmuth Ruthenium Oxide Resistor

Bismuth Ruthenium Oxide Resistor

Bismuth Ruthenium Oxide Resistor

Bismuth Ruthenium Oxide Resistor

Bismuth Ruthenium Oxide Resistor

Reller PSI Series 400/Silicon Diode

Keller PSI Sexies 400/Silicon Diode

Keller PSI Serles 400/silicon Dlode Keller PSI Serles 400/silicon Diodo Keller PST Series 400/silicon Diodo Keller pSI Series 400/silicon Diode Reller PSI Series 400/silicon Diode Kellar PST Series $400 /$ silicon Diodo Kellex PSI Sertes 400/silicon Diode Keller PSI Series 400/Silicon Diode
Operating Point Operating Range

$80 \mathrm{~K} \quad 300,90-50 \mathrm{~K}$

$40 \mathrm{~K} \quad 300,90-25 \mathrm{~K}$

$300,90-50 \mathrm{~K}$

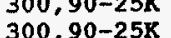

$300,90-12 \mathrm{~K}$

$300,90-25 \mathrm{~K}$

$300,90-25 \mathrm{~K}$
$300,90-12 \mathrm{~K}$

$300.90-15 K$

$300,90-15 K$

$300,90-50 \mathrm{~K}$

$300,90-50 \mathrm{~K}$

$300,90-70 \mathrm{~K}$

$300,90-70 \mathrm{~K}$

$300,90-60 \mathrm{~K}$

$300,90-60 \mathrm{~K}$

$300,90-15 \pi$

$300,90-15 \mathrm{~K}$

$300,90-15 K$

$300,90-15 K$

$300,90-15 K$

$300,90-15 K$

$300-12 K$

$300-4 K$

$300-4 \mathrm{~K}$

$300-4 K$

$300-4 \mathrm{~K}$

$37.5-77 \mathrm{~K}$

$37.5-77 \mathrm{~K}$
$37.5-77 \mathrm{~K}$

$37.5-77 \mathrm{~K}$

$37.5-77 \mathrm{~K}$

$300-60 \mathrm{~K}$

$300-60 \mathrm{~K}$

$300-60 \mathrm{~K}$

$300-60 \mathrm{~K}$

300,90-50K

$300,90-25 \mathrm{~K}$

$300,90-50 \mathrm{~K}$

$300,90-25 \mathrm{~K}$

300,90-25K

$300,90-12 K$

$300,90-25 K$ $300,90-12 \mathrm{~K}$

TBD

$300,90-25 \mathrm{~K}$

$300,90-25 K$

$300,90-25 R$

300,90-15K

TBD

TBD

$300,90-15 \mathrm{~K}$

$300,90-77 K$ 
Table 2. List of All Sensors Used in the Data Acquisition (Continued)

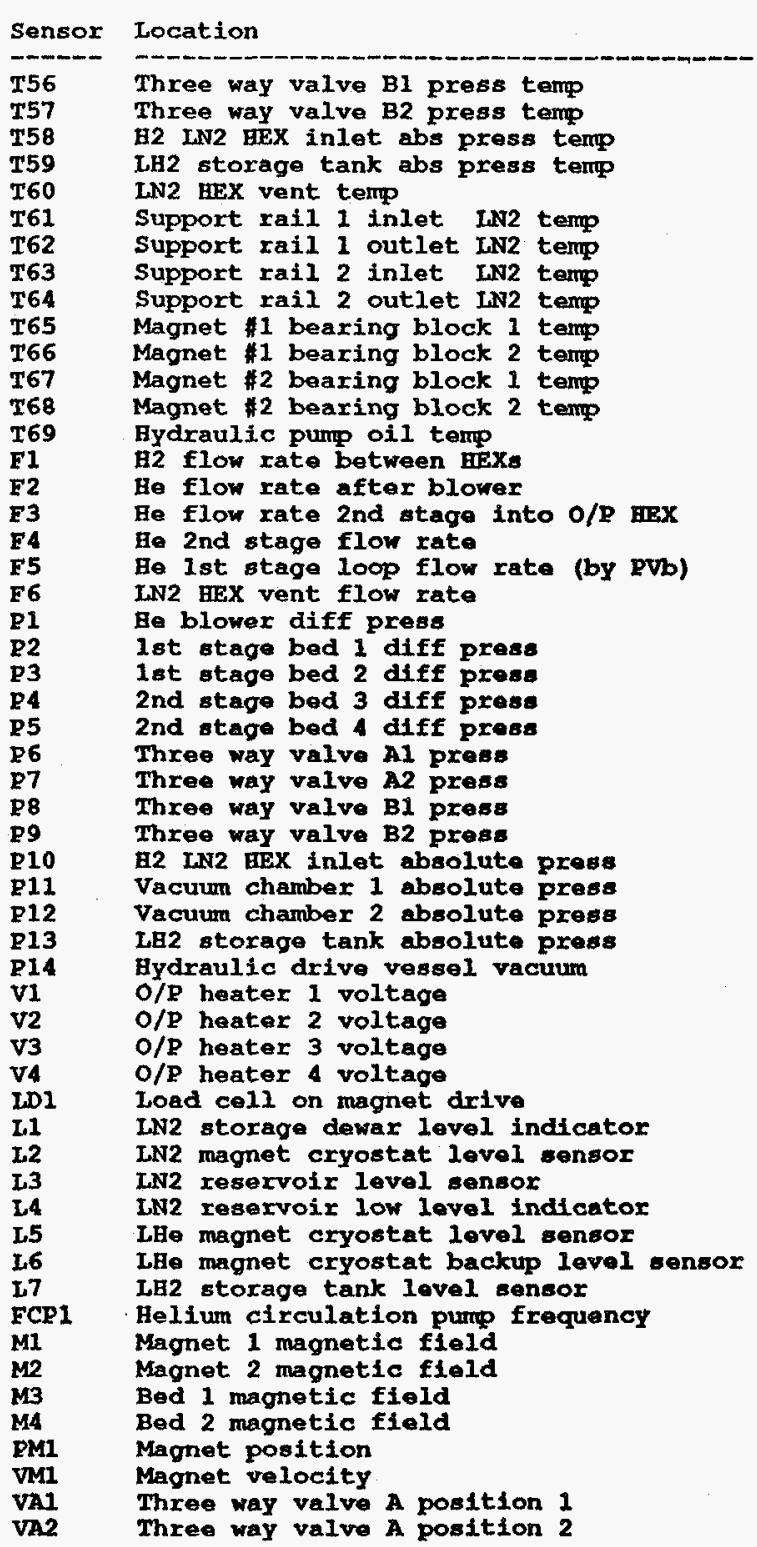

Type

Kellex PSI Series 400/Silicon Diode Keller PSI Series 400/Silicon Dioda keller PSI Series 400/sillcon Diode Platinum Resistor

Platinum Resistor

Pratinum Resistor

Platinum Resistor

Platinum Resistor

Platinum Resistor

Platinum Resistor

platinum Resistor

Platinum Resistor

TBD - was Micromotion DS012s Cortolis

TBD - Was Micromotion DS150s Coriolis

TBD - was Micromotion DS025S Coriolis

TBD - was Micromotion DSIO0S Coriolis

TBD

Keller PSI Series 400 Cryogenic

Keller PSI Series 400 Cryogenic

Kellex PSI Series 400 Cryogenic

Kallex PSI Series 400 Cryogenic

Keller PSI Serles 400 Cryogenic

Kellex PSI Series 400 Cryogenic

Keller PSI Series 400 Cryogenia

Teller PSI Series 400 Cryogento

Tellar PSI Sexion 400 Cryogenic

Varian Ionization Gage

Varian Ionization Gago

Keller PSI Series 400 Cryogenic

TBD

N/A

N/A

$\mathrm{N} / \mathrm{A}$

Eaton/Lebow

TBD - Was Keller Series 400 Level

TBD - Was Kellex Series 400 Level

TBD - Has Kellex Series 400 Level

IBD - Was Kellex Serfes 400 hevel

TRD - Was Kellex Sertes 400 Lovel

2BD - Was Kellex Sarias 400 Level

TBD

TBD

F.W. Bell Hall Probe

F.W. Bell Hall Probe

.W. Bell hall Probe

Temposonics Magnetostrictive Device

TBD

Shunt
Shunt
$>80 \mathrm{~K}$

$313-240 \mathrm{~K}$

$>20 \mathrm{~K} \quad 300,20 \mathrm{~K}$

$\begin{array}{ll}77 \mathrm{~K} & 300-70 \mathrm{~K} \\ 77 \mathrm{~K} & 300-70 \mathrm{~K}\end{array}$

$\begin{array}{ll}77 \mathrm{~K} & 300-70 \mathrm{~K} \\ 7 \mathrm{~K} & 300-70 \mathrm{~K}\end{array}$

$77 \mathrm{~K} 300-70 \mathrm{~K}$

$77 \mathrm{~K} \quad 300-70 \mathrm{~K}$

$\begin{array}{ll}4-77 \mathrm{~K} & 4-77 \mathrm{~K} \\ 4-77 \mathrm{~K} & 4-77 \mathrm{~K}\end{array}$

$4-77 \mathrm{~K} \quad 4-77 \mathrm{~K}$

$>300 \mathrm{~K}$

$1 \mathrm{~g} / \mathrm{s} \quad 0-10 \mathrm{~g} / \mathrm{s}$

$436 \mathrm{~g} / \mathrm{s}$

$10 \mathrm{~g} / \mathrm{s}$

$77 \mathrm{~g} / \mathrm{s}$

$349 \mathrm{~g} / \mathrm{s}$

$27 \mathrm{~g} / \mathrm{s}$

15 psid
2.8 psid

$2.8 \mathrm{psid}$
$2.8 \mathrm{psid}$

0.6 psid

0.6 psid

285-300 psia

$285-300$ psia

285-300 psia

600 psia

600
$\mathrm{E}-7$ toria

E-7 torx

15 psia

1 psid

$0-10$ VDC
$0-10$ VDC

$0-10$ VDC
$0-10$ VDC

$0-10$ VDC

$0-10$ VDC
+-4900 Jbe

$+-4$

40-100 cm

$51 \mathrm{~cm}$

14-35 cm

$14-35 \mathrm{~cm}$
$14-35 \mathrm{~cm}$

$14-35$

TBD

7 T

$7 \mathrm{~T}$

5 T

$0-46 \mathrm{~cm}$

$+-142 \mathrm{~cm} / \mathrm{s}$

$0-5$ VDC
$0-5$ VDC
$0-20 \mathrm{~g} / \mathrm{s}$

$0-100 \mathrm{~g} / \mathrm{s}$

$0-500 \mathrm{~g} / \mathrm{s}$

$0-50 \mathrm{~g} / \mathrm{s}$

$0-20$ psid

$0-5$ psid

$0-2$ paid

0 - 2 psid

$0-2$ psid

$0-350$ psia
$0-350$ poia

$0-350$ psia

$0-350$ psia

0-700 psia

$E-4>E-8$ tor

TBD

$0-5$ psid

$0-10$ vDC

$0-10$ VDC

$0-10$ VDC

$0-10$ VDC
$+-100001 \mathrm{be}$

TBD

$0-100 \mathrm{~cm}$

$43-58 \mathrm{~cm}$
$25-35 \mathrm{~cm}$

$0-35 \mathrm{~cm}$

$0-35 \mathrm{~cm}$
$0-35 \mathrm{~cm}$

TBD

64-215 B

$0-8 \mathrm{~T}$

$\begin{array}{ll}0-8 & T \\ 0-8 & T\end{array}$

$\begin{array}{lll}0-8 & T \\ 0-8 & T\end{array}$

$0-8 \mathrm{~T}$

$0-46 \mathrm{~cm}$

$+-142 \mathrm{~cm} / \mathrm{s}$

$0-5$ VDC
$0-5$ VDC.
$0-500 \mathrm{~g} / \mathrm{s}$ 
Table 2. List of All Sensors Used in the Data Acquisition (Continued)

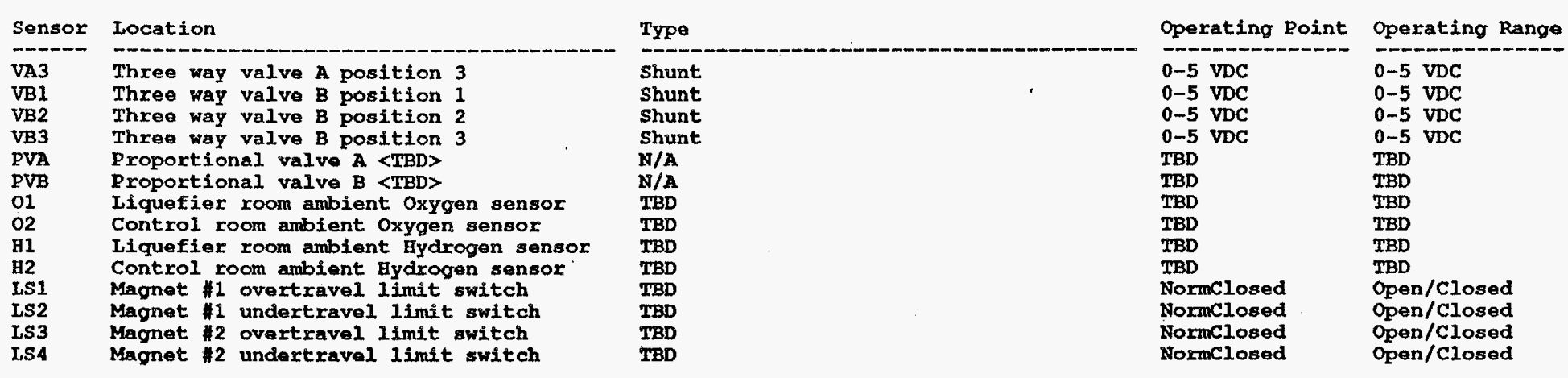




\subsection{Test Results}

\subsubsection{Test Results}

\subsubsection{1. $\mathrm{Er}_{0.86} \mathrm{Gd}_{0.14} \mathrm{Al}_{2}$ Test Results}

AMR tests were run for discrete values of the magnetic field of $0.5,1.0,2.0,3.0$, 5.0 , and $7.0 \mathrm{~T}$. The operating temperature range was $10-20 \mathrm{~K}$. The objective of running the experiments was to find cooling loads under various magnetic fields at different CHEX temperatures. Other experimental goals were to achieve different loads as a function of magnetic field and to compare experimental results with the model predictions.

The magnetic material $\mathrm{Er}_{0.86} \mathrm{Gd}_{0.14} \mathrm{Al}_{2}$ has been employed in the refrigerator. Ground and sieved magnetic material was employed for the initial tests. Bed length is $0.09 \mathrm{~m}$ with a cross section of $0.000284 \mathrm{~m}^{2}$. Particle sizes range from 0.0002 and $0.0004 \mathrm{~m}$. The porosity is 0.44 .

Figure 17 shows the adiabatic temperature change of the material from zero field to the field indicated and from the field indicated back to zero field. The temperature on the abscissa represents the lower temperature of a magnetization or demagnetization measurement. These results are obtained from our own 'heat capacity' apparatus in which both heat capacity and adiabatic temperature change data can be obtained for a sample.

Note that the points are typically double-valued. The lower of the two points shown for a given measurement represents demagnetization. A small amount of magnetic hysteretic heating is consequently indicated by the data. To determine directly the impact of hysteretic heating, the AMR was run with only field change (no flow) on this material. A steady rise in temperature consistent with the data shown in Figure 17 is observed, confirming that hysteretic heating observed in the heat capacity apparatus is observed in the AMR. Figure 17 indicates that the material has an adiabatic temperature change which is close to being proportional to the absolute temperature over the $10 \mathrm{~K}$ to $20 \mathrm{~K}$ temperature range.

Figure 18 shows an experimental run at a field of $3.0 \mathrm{~T}$, from startup. The period for the total AMR cycle is $10 \mathrm{~s}$ (cold/hot flow periods are $2.5 \mathrm{~s}$; field change periods are $2.5 \mathrm{~s}$ ) here, and for all data shown in this report. Figure 18 indicates the HHEX and CHEX temperatures were both at about $14.5 \mathrm{~K}$, prior to starting the AMR cycle. After startup, the temperature difference is rapidly established. The CHEX achieves its bottom temperature of about $9 \mathrm{~K}$ after only about seven cycles. The HHEX is continuing to rise throughout the 14 cycles shown. The displacer motion, per blow, is 
$0.015 \mathrm{~m} / \mathrm{s}$ for $3.3 \mathrm{~s}$. The very rapid approach to steady state, with respect to the number of AMR cycles, indicates that heat capacity of the total gas flowing through the bed, per blow, is comparable to the total heat capacity of the magnetic material in the bed.

It has been demonstrated that the beds and the CHEX are thermally isolated from the liquid helium dewar. With no displacer motion or magnet motion, the temperatures in the bed and CHEX remain constant with or without helium in the dewar. With displacer motion and no magnet motion, the beds and CHEX achieve the temperature of the HHEX.

Figure 19 shows another $3 \mathrm{~T}$ run from the same series as that in Figure 18. That is, the same initial experimental conditions apply. The temperature sensors in the middle of the bed and in the filter between the bed and HHEX are shown. The displacer motion here is $0.02 \mathrm{~m} / \mathrm{s}$ for a period of $1 \mathrm{~s}$. Less than one-third of the gas flows through each bed here compared to the run in Figure 18.

Figure 20 shows yet another $3 \mathrm{~T}$ run, after steady state has been reached. The initial experimental conditions of this run are different than that of the runs shown in Figures 18 and 19. As well as the HHEX and CHEX temperatures, the temperature sensors in the middle of the bed and in a filter between the bed and HHEX are shown. The displacer motion here is $0.02 \mathrm{~m} / \mathrm{s}$ for a period of $0.75 \mathrm{~s}$. A total no-load temperature span from approximately $20.5 \mathrm{~K}$ to $7.25 \mathrm{~K}$ is indicated.

Figure 21 shows a $5 \mathrm{~T}$ run with no load, after steady state. The full five temperature sensors in the bed (axial locations $0.0,0.0125,0.045,0.0675$, and $0.09 \mathrm{~m}$ from the cold end of the bed) are displayed in Figure 22. The displacer motion is $0.018 \mathrm{~m} / \mathrm{s}$ for $0.5 \mathrm{~s}$.

\subsubsection{2. $\mathrm{GdNi}_{2}$ Test Results}

AMR tests were run for discrete values of the magnetic field of $3.0,5.0$, and 7.0 $\mathrm{T}$. The operating temperature range was $25-90 \mathrm{~K}$. The chief objective of running these tests was to find the cooling load at $40 \mathrm{~K}$ with the HHEX temperature at $77 \mathrm{~K}$. Other experimental goals were to achieve different loads as a function of magnetic field, and compare experimental results with the model predictions.

Magnetic material $\mathrm{GdNi}_{2}$ has been employed in the refrigerator. Ground and sieved particles in the size range of $0.0001 \mathrm{~m}$ to $0.0002 \mathrm{~m}$ were used. The cold and hot flow periods were $3 \mathrm{~s}$ and the field change period was $2 \mathrm{~s}$. This gives a cycle period of $10 \mathrm{~s}$, which was held constant throughout the experiments. The gas pressure ranged from 1 atm to 4 atm. 
Table 3 summarizes the test conditions for $7 \mathrm{~T}$ runs of $\mathrm{GdNi}_{2}$ bed material. Twenty-four operating points were obtained by varying mass flow rate, cooling load, and resulting CHEX temperature. Each row represents one operating point. The columns indicate: number of data file, helium circuit mean gauge pressure, helium displacer motion distance, HHEX temperature, bed hot end gas temperature, measured in an in-line filter between the HHEX and the lower bed, load power, and resulting CHEX temperature.

HHEX and CHEX temperatures versus time, plotted in Figure 23, are the results of a typical $7 \mathrm{~T}$ run. After adjusting the HHEX temperature to $75 \mathrm{~K}$, the AMR was operated under no-load conditions lowering the CHEX temperature to $28 \mathrm{~K}$. The CHEX temperature was stabilized before any power was applied to the CHEX. A load $(0.8 \mathrm{~W}-1.6 \mathrm{~W})$ was incremented in small steps of $0.4 \mathrm{~W}$, allowing the CHEX temperature to stabilize after each power increase. During typical operating point tests, one reading of all measured values was taken every two minutes. These "slow speed" measurements gave sufficient information to observe the trends toward equilibrium values. 
Table 3. Summary of $\mathrm{GdNi}_{2}$ Test Runs at $7 \mathrm{~T}$ Magnetic Field

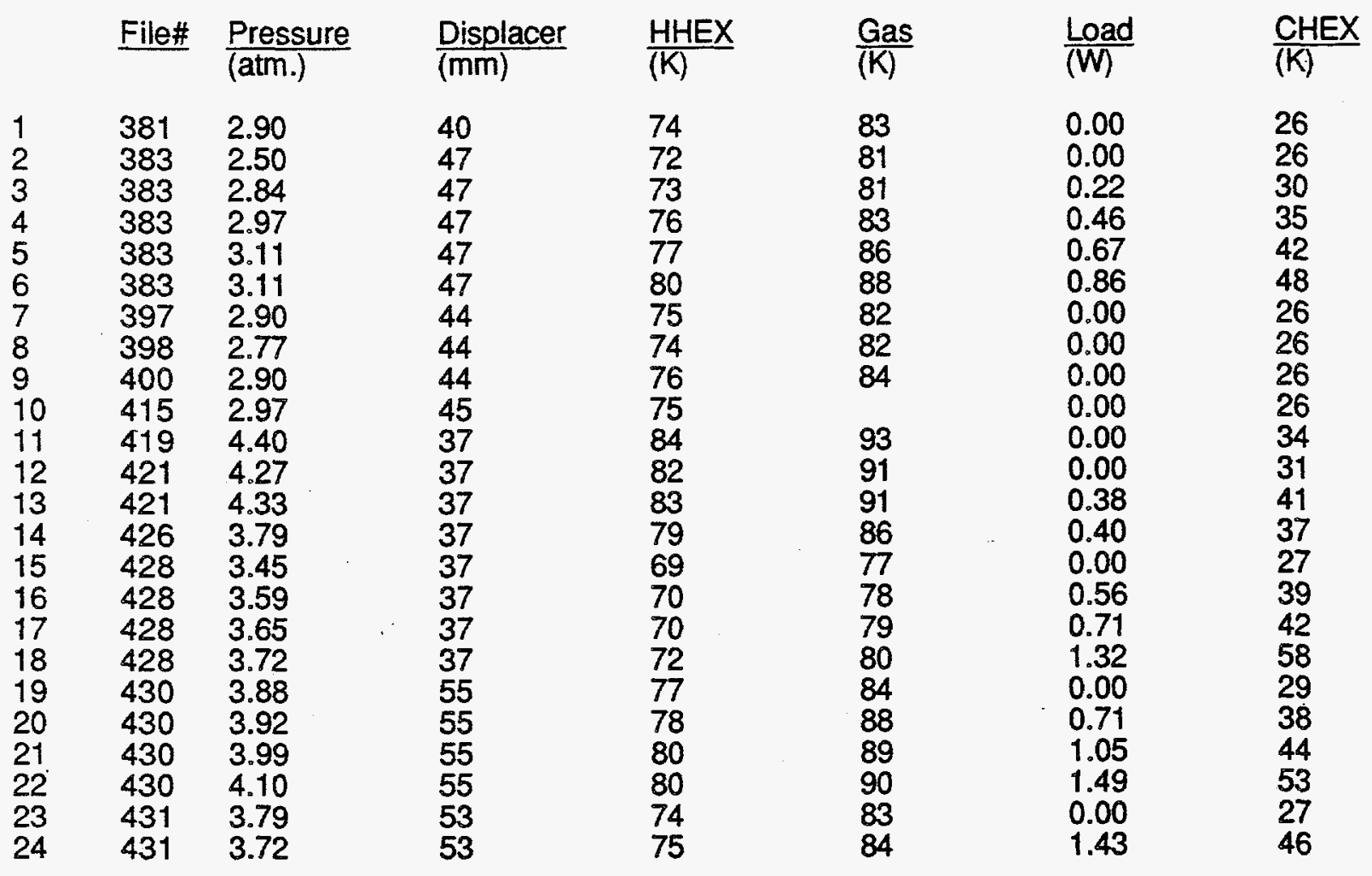




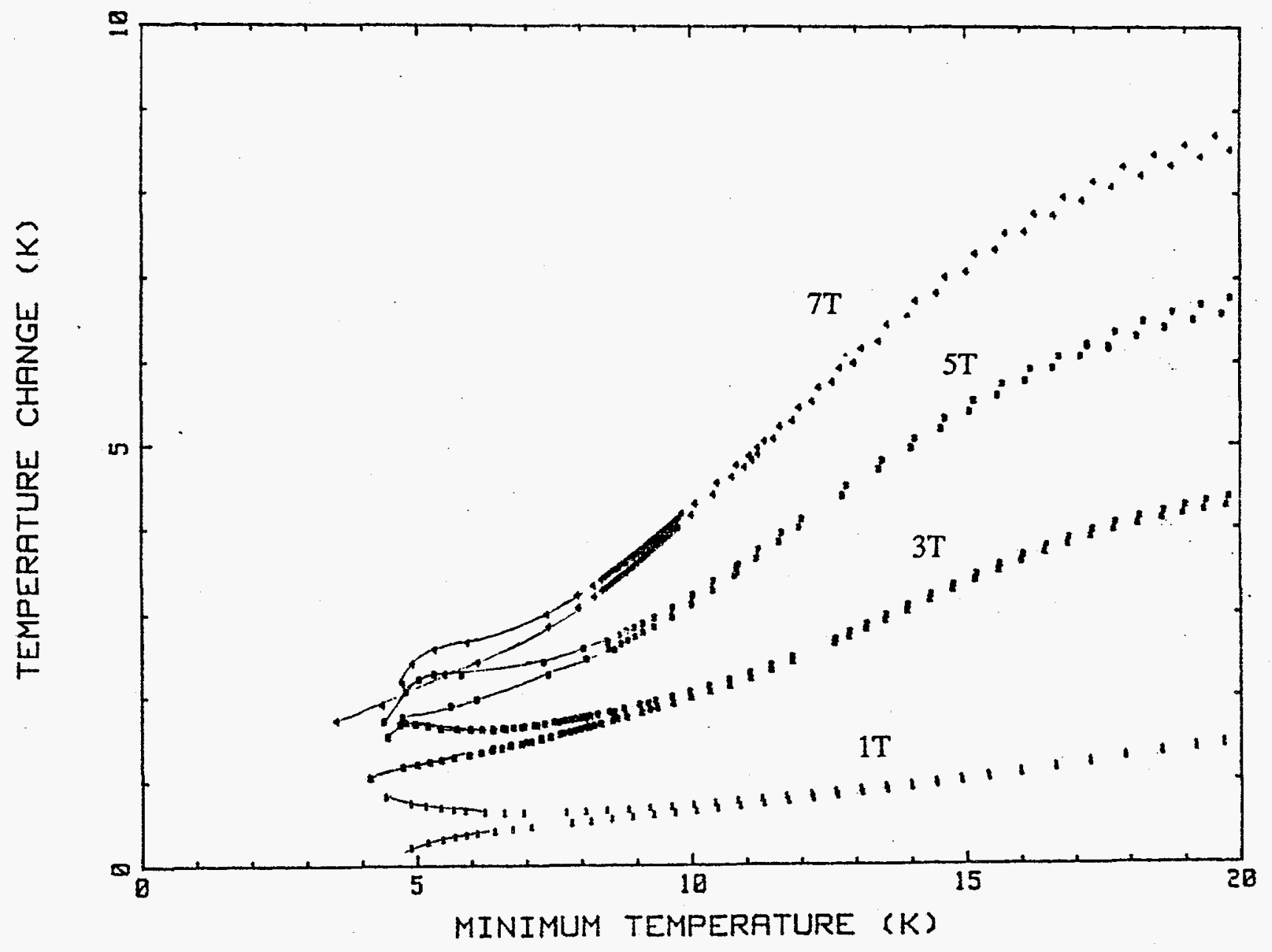

Figure 17. Adiabatic Temperature Change of $\mathrm{ErGdAl}_{2}$ Bed Material 


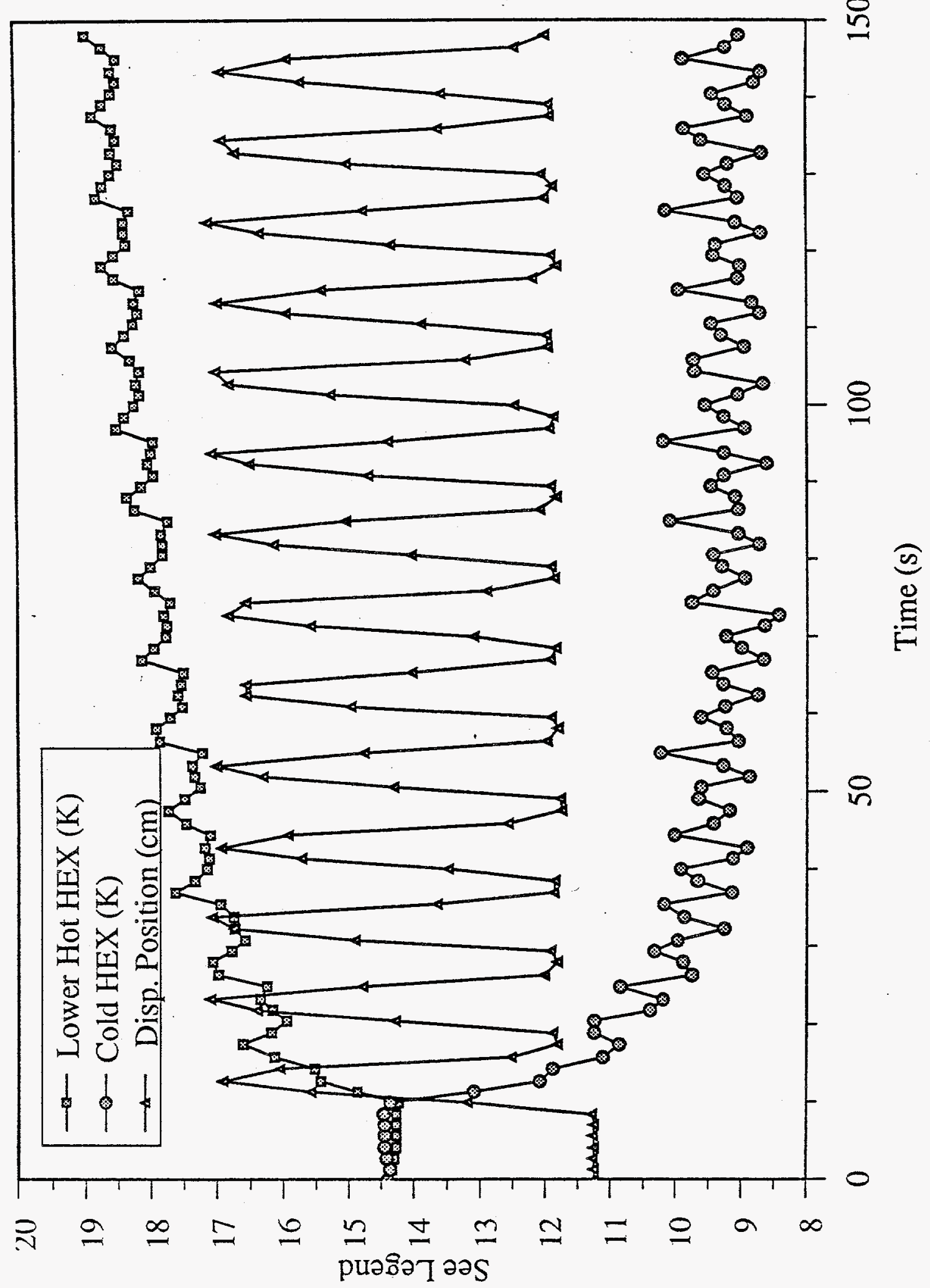

Figure 18. HHEX and CHEX Temperature vs. Time $\left(\mathrm{Er}_{0.86} \mathrm{Gd}_{0.14} \mathrm{Al}_{2}\right)$ 3 T, Displ. Superimposed 


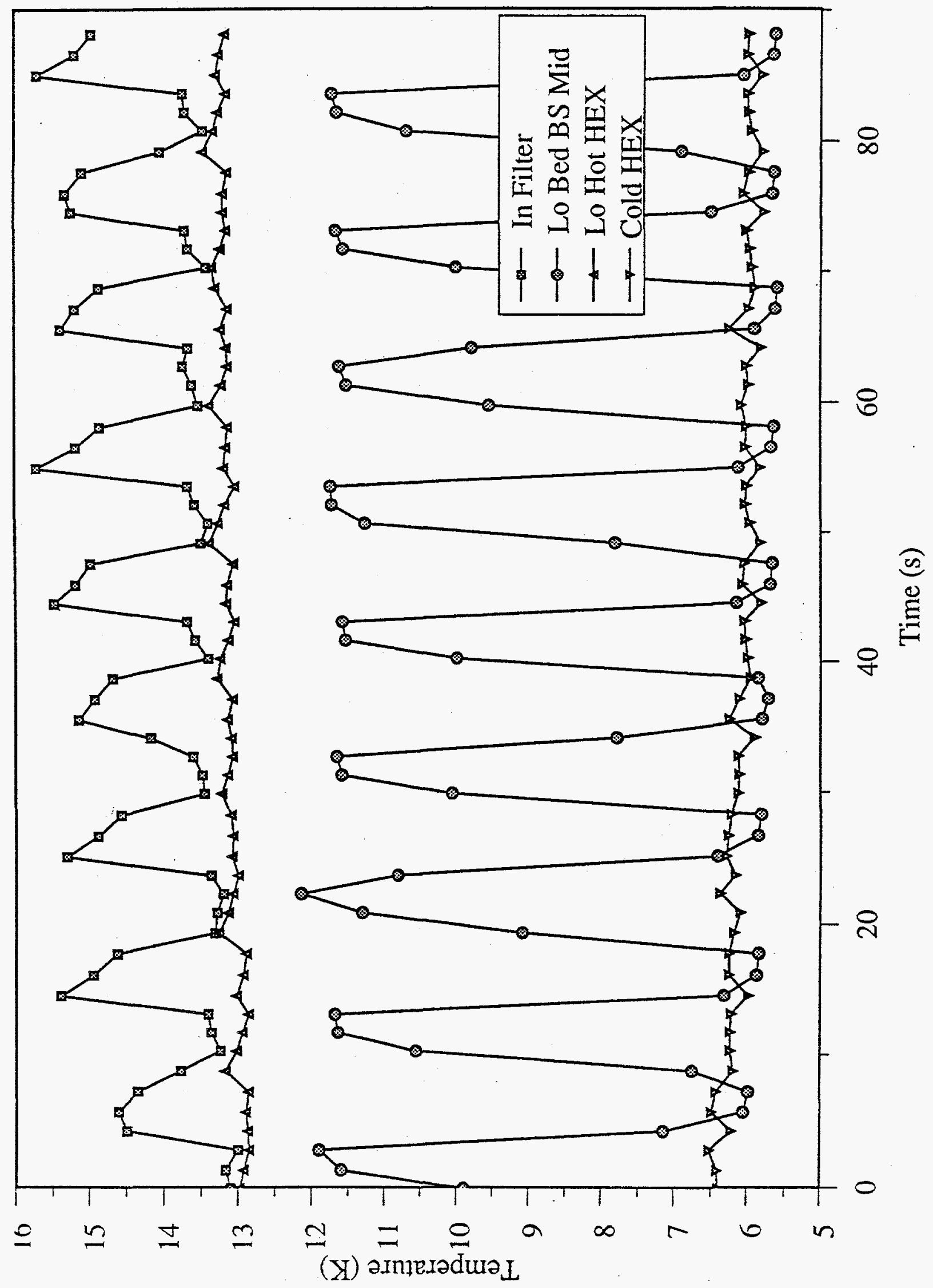

Figure 19. HHEX and CHEX Temperature vs. Time $\left(\mathrm{Er}_{0.86} \mathrm{Gd}_{0.14} \mathrm{Al}_{2}\right), 3 \mathrm{~T}$ 


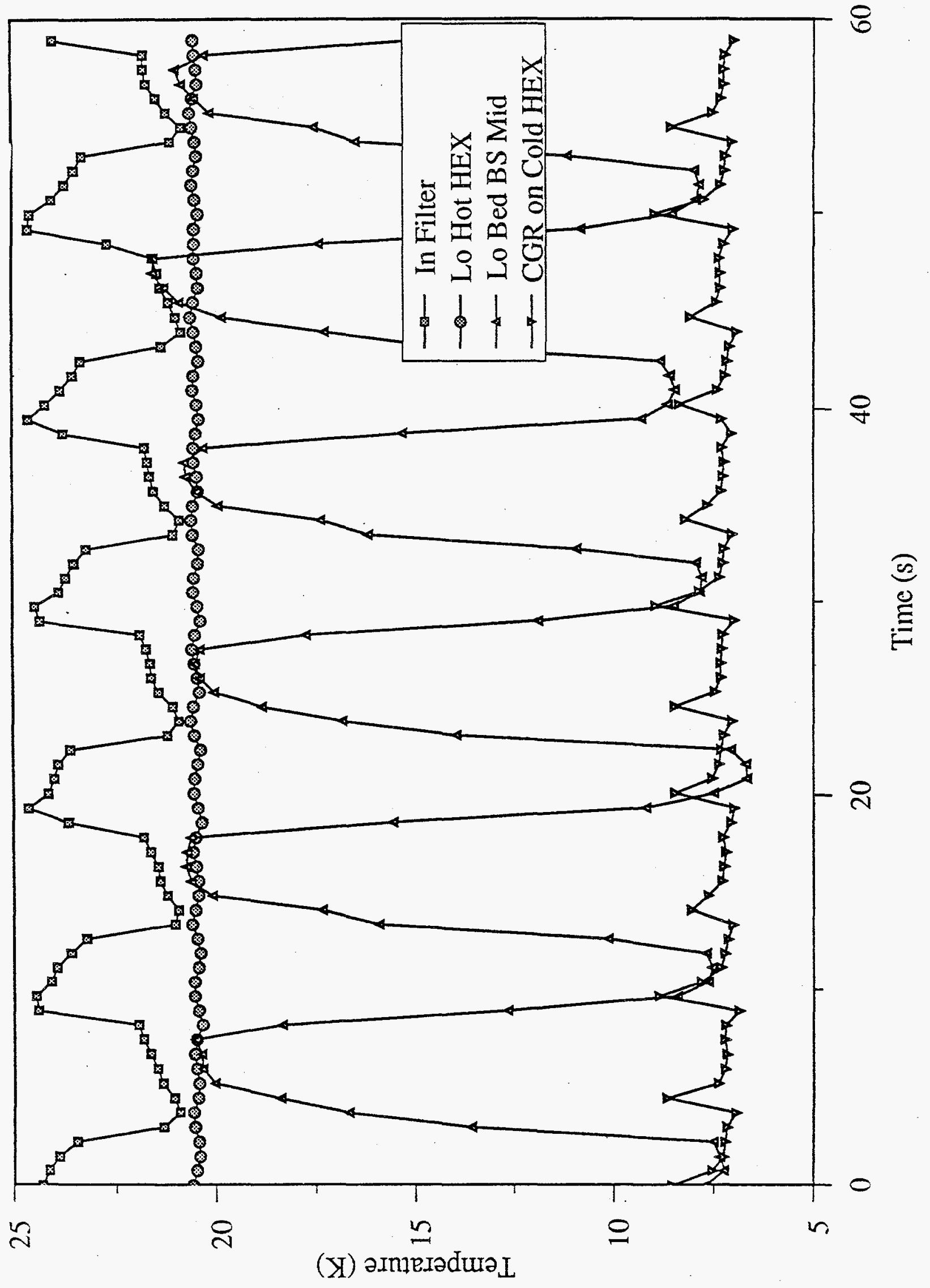

Figure 20. HHEX and CHEX Temperature vs. Time $\left(\mathrm{Er}_{0.86} \mathrm{Gd}_{0.14} \mathrm{Al}_{2}, 3 \mathrm{~T}\right)$ 


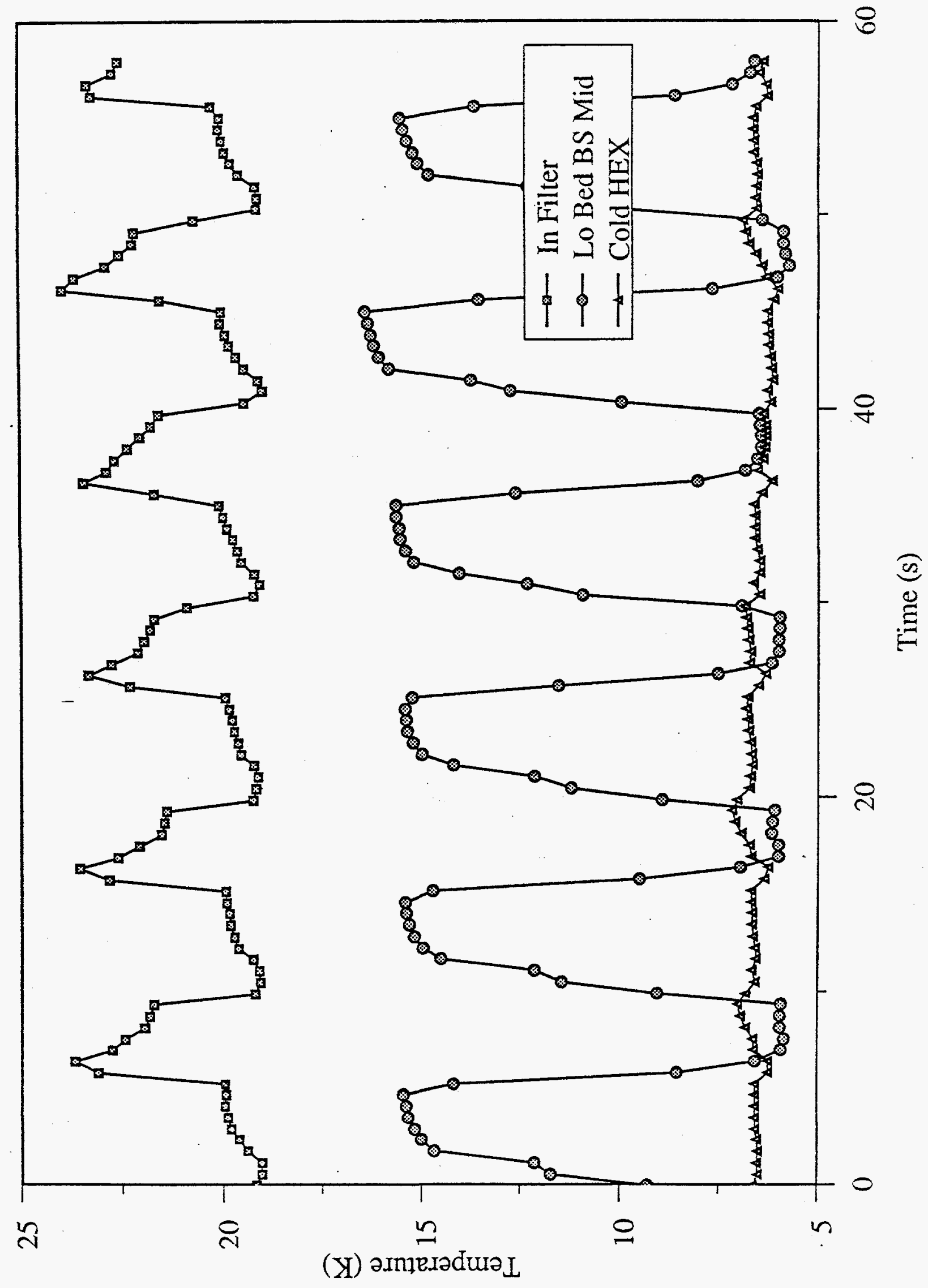

Figure 21. CHEX, Middle Bed, and In Filter Temperatures vs. Time $\left(\mathrm{Er}_{0.86} \mathrm{Gd}_{0.14} \mathrm{Al}_{2}\right)$ 


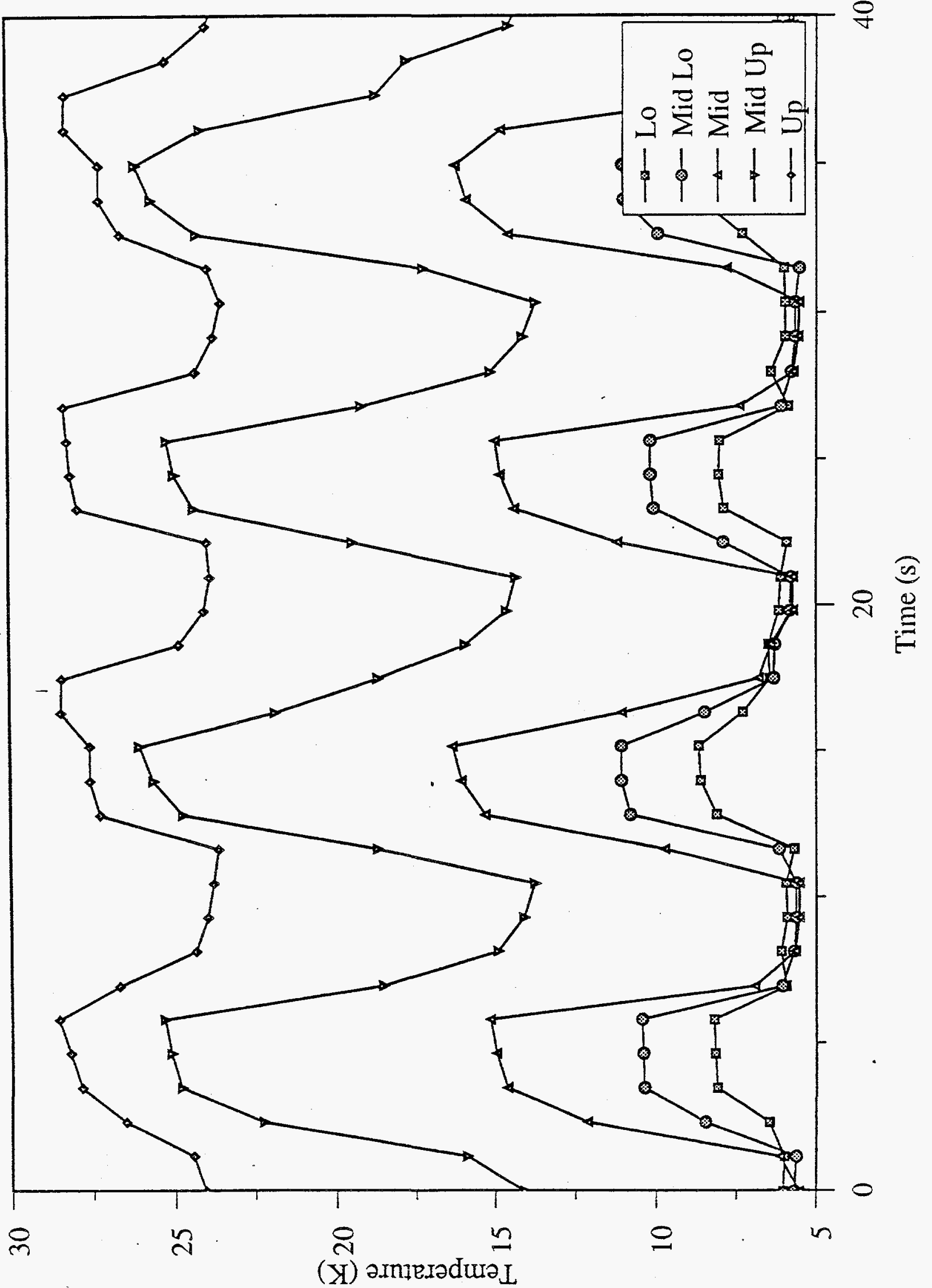

Figure 22. Temperature in Bed at Five Axial Locations vs. Time $\left(\mathrm{Er}_{0.86} \mathbf{G d}_{0.14} \mathrm{Al}_{2}\right)$ 
To observe operation in detail, higher speed measurements were taken. These measurements recorded the value of a single channel sampled approximately three times each second, and confirmed the apparatus was executing the refrigeration cycle desired. A set of these readings taken under steady state conditions indicated by run number 421 at $0.4 \mathrm{~W}$ (see Table 3) is shown in Figures 24-30.

The displacer position as a function of time, shown in Figure 24, indicates that the motion is very steady during the blow period and nearly stationary during magnet motion. The magnet position as a function of time, plotted in Figure 25, indicates that the change in position is executed within $2 \mathrm{~s}$ and the magnet is practically stationary for $3 \mathrm{~s}$ during the blow.

Figures 26 and 27 present differential pressure readings taken across portions of the helium gas circuit. A pressure transducer, located between the HHEX and bed, measures the pressure across a flow impedance, as shown in Figure 26, serving as an uncalibrated flow meter. This indicates that the flow rises quickly from zero to some nearly steady value during the blow phase, and drops rapidly to zero, where it remains during magnet motion. The flow exhibits good symmetry. Figure 27 is a similar plot of pressure across the displacer, or equivalently across the entire circuit.

Additional observations not digitally recorded confirm that the cycle was wellexecuted by the apparatus. The actuator control computer plot and direct observation indicate that the motions of displacer and magnet were non-overlapping and had the desired wave shape. An analog dial pressure gauge measuring one side of the displacer relative to room atmosphere was used to determine the mean pressure. The needle of this gauge would move above or below the mean by half of the total pressure drop during displacer motion, and return to the mean before the magnet began moving. This indicates completion of the flow while the magnet is stationary.

Figure 28 shows the temperature of a sensor suspended in a particle filter, located between the bed assembly and the HHEX, versus time. This sensor was used as an indication of the bed hot end temperature. Using the experiment, the bed hot end temperature was typically significantly higher than the HHEX temperature, which indicates reduced HHEX effectiveness at higher mass flow rates. The periodic temperature fluctuations of this thermometer should reflect the adiabatic temperature change of the hot end of the bed, but they are significantly lower. Thermal damping by the copper tube may have limited the fluctuation. A new bed assembly to be tested will put sensors proximal to both hot end exits.

Figure 29 shows the temperature near the exit of the displacer as a function of time. The temperature is higher than the HHEX temperature and shows a periodic fluctuation at twice the operating frequency because of the heat input due to displacer seal friction. 


\section{GdNi2 A.M.R.}

7 Tesla, 2 Atm., 10 sec. period.

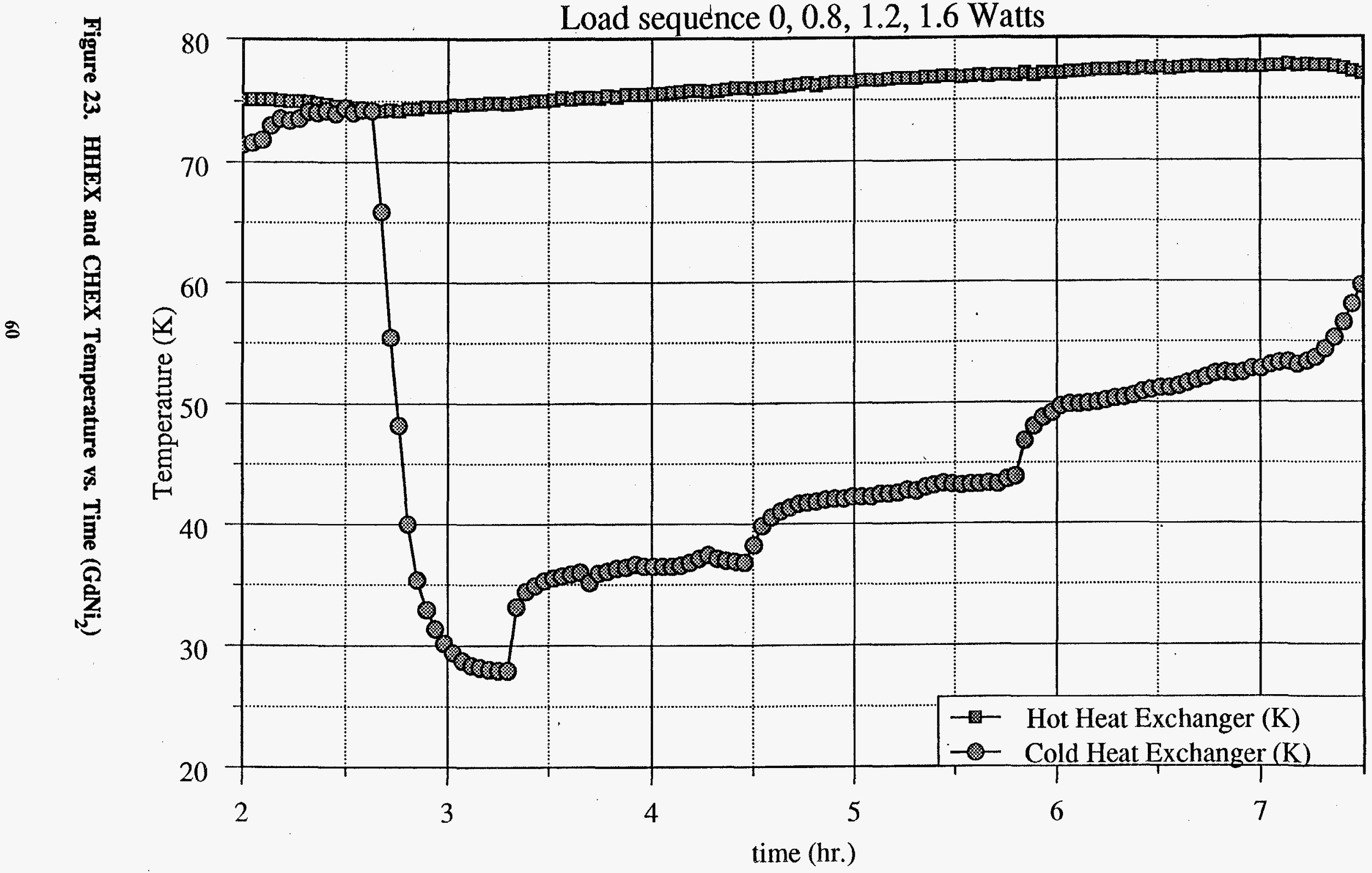




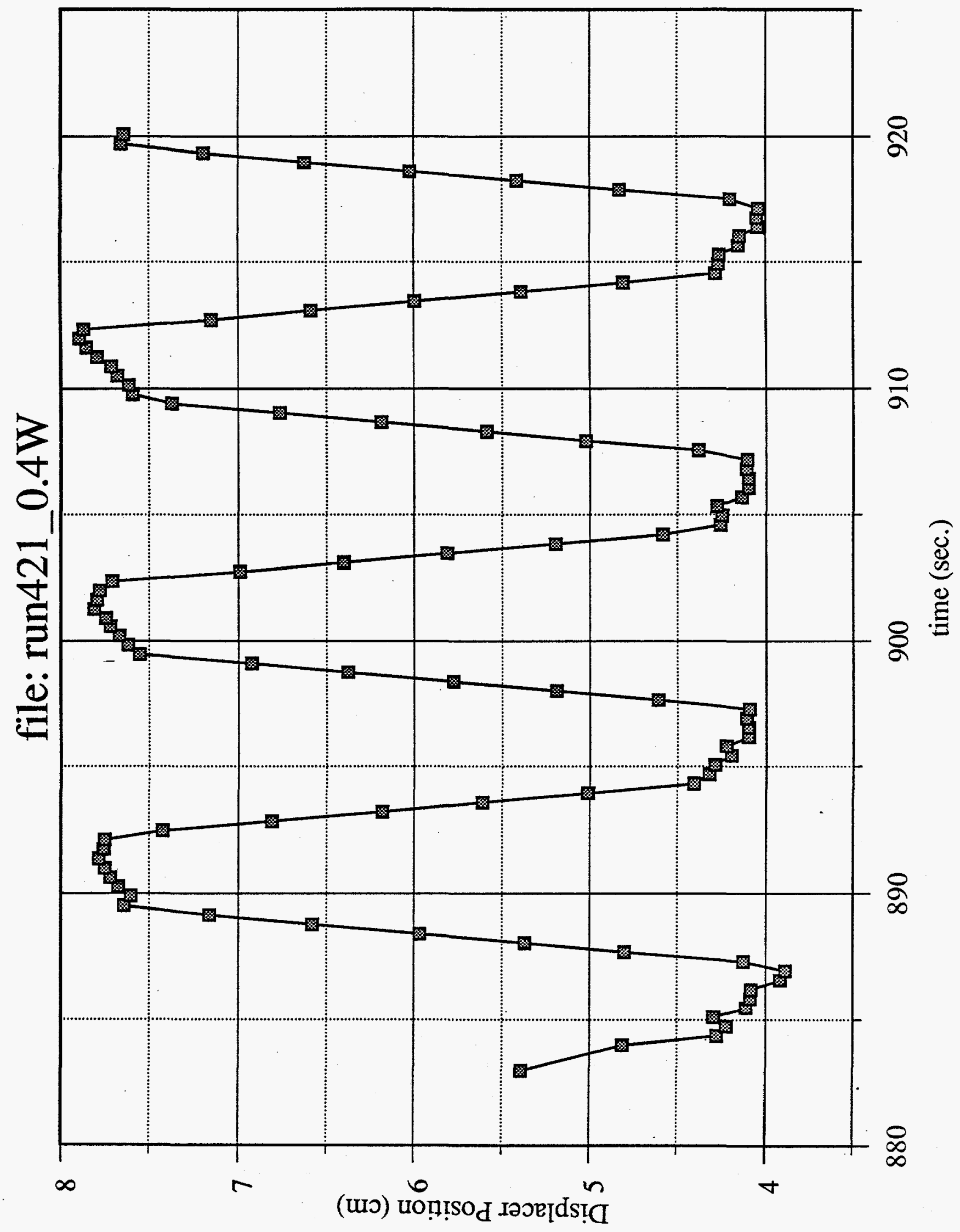

Figure 24. Displacer Position vs. Time 


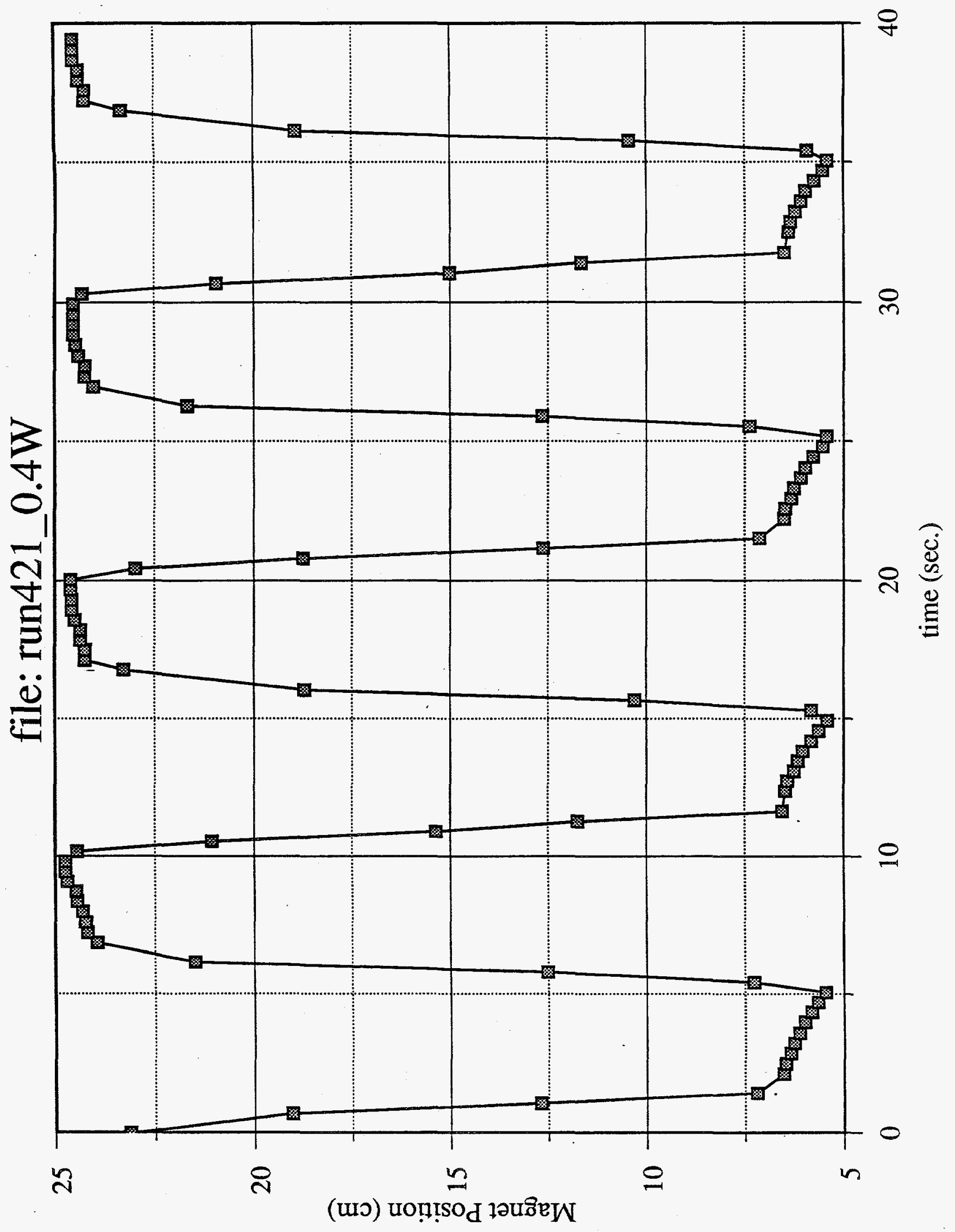

Figure 25. Magnet Position vs. Time 


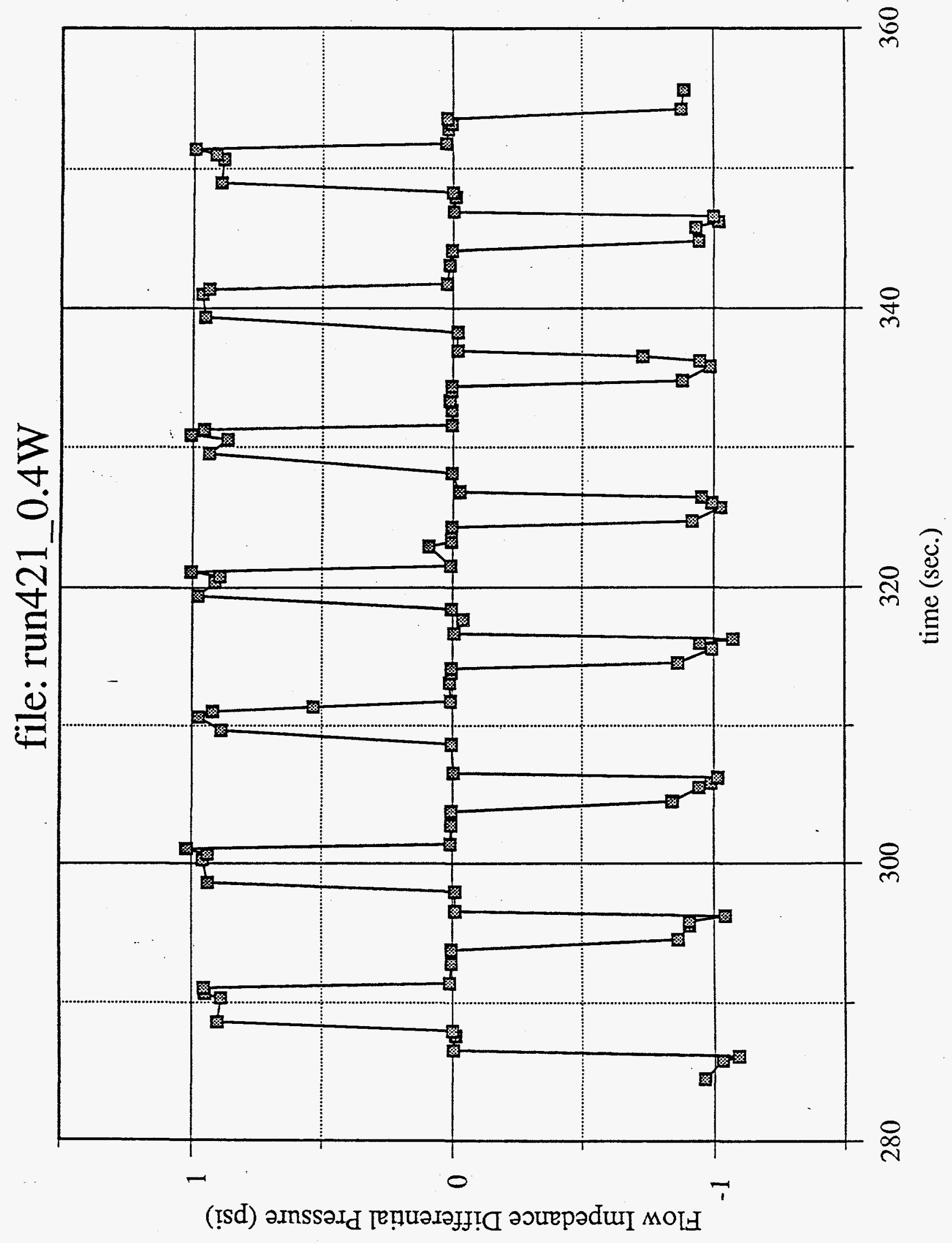

Figure 26. Flow Impedance Differential Pressure vs. Time 


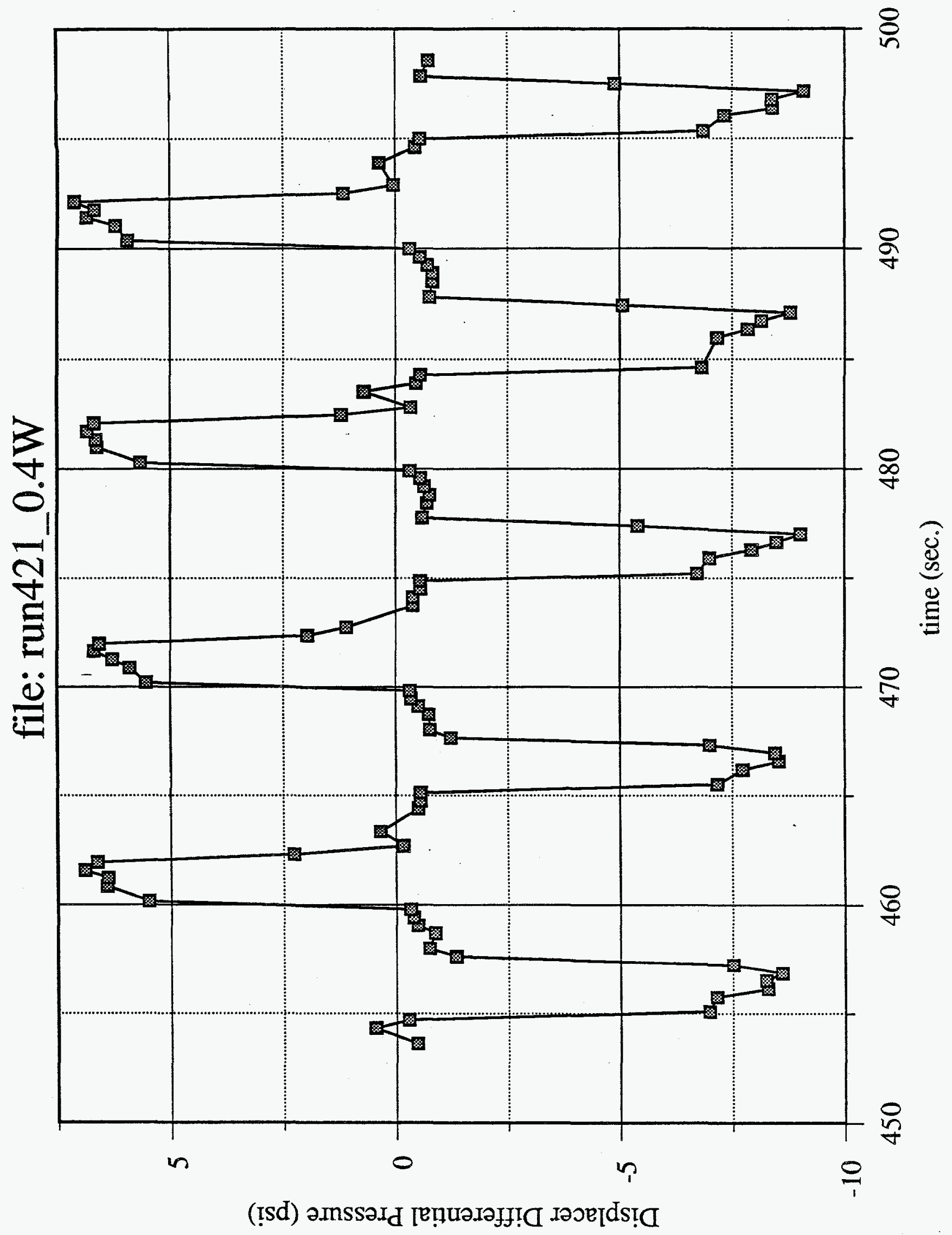

Figure 27. Displacer Differential Pressure vs. Time 


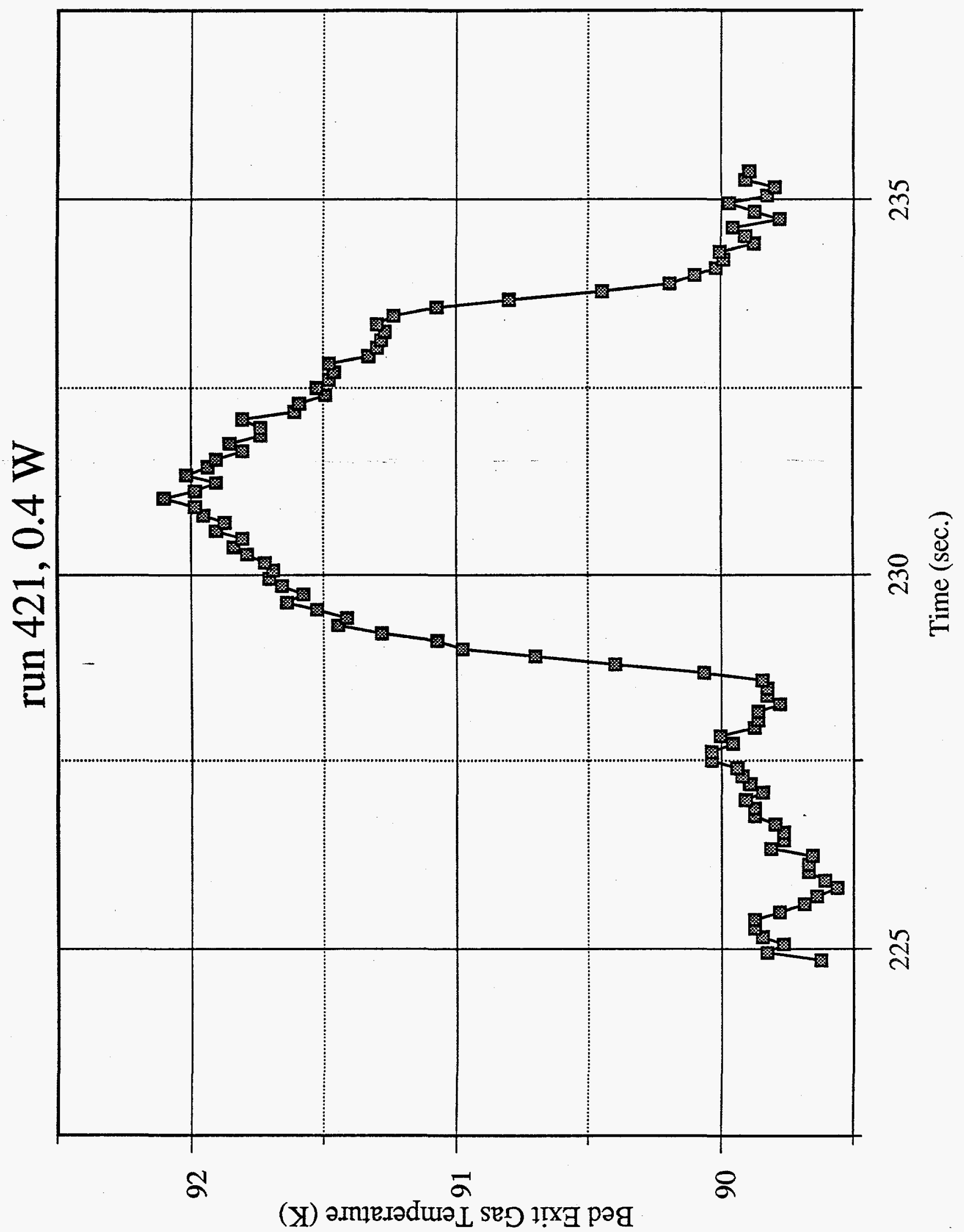

Figure 28. Gas Temperature at Bed Exit vs. Time 


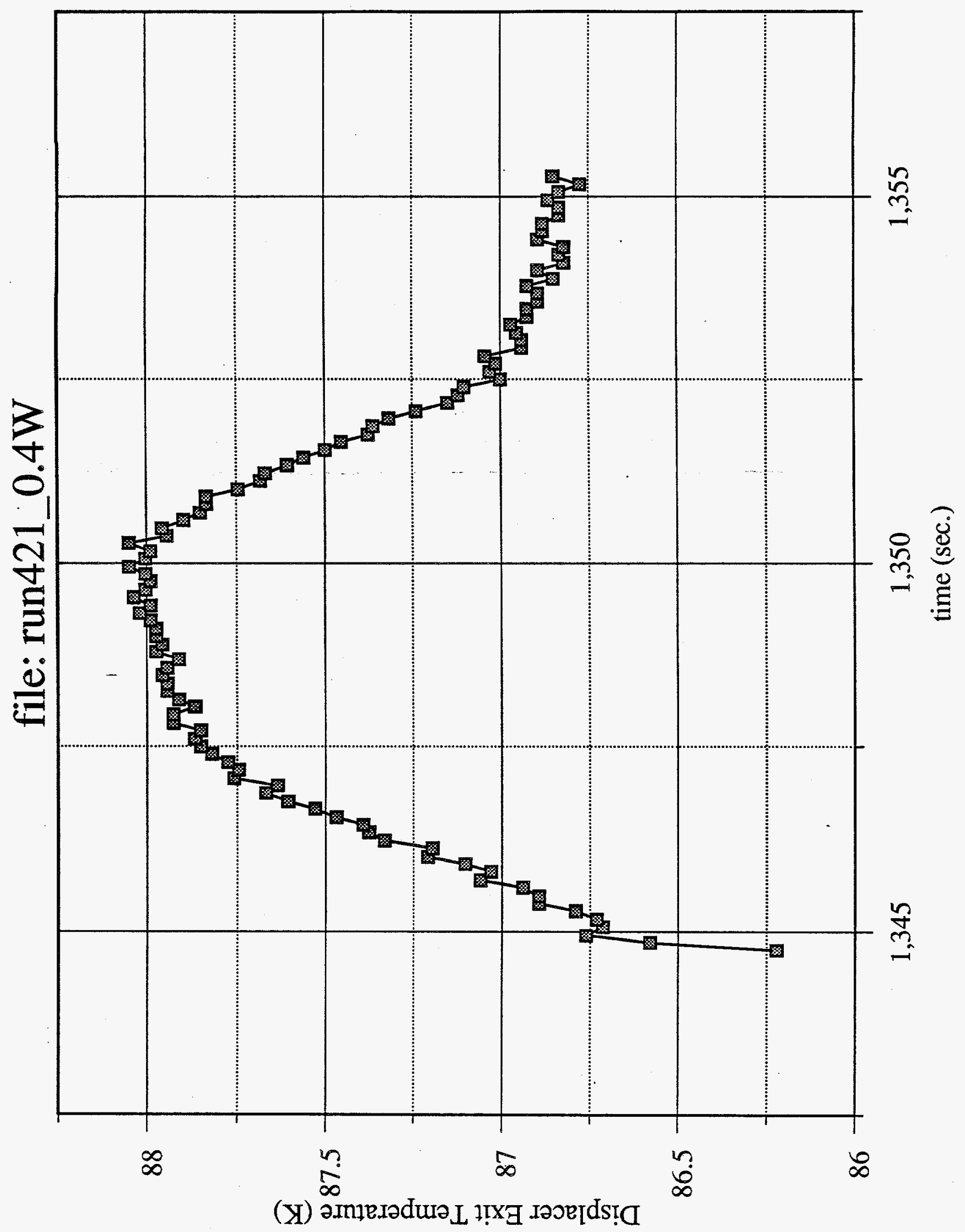

Figure 29. Displacer Exit Temperature vs. Time 
Figure 30 shows the magnet load cell force versus time. When the magnet is stationary the force is steady near $100 \mathrm{lbs}$ in the upper position or $0 \mathrm{lbs}$ in the lower position due to a spring used to counter some of the weight. Large peaks and variability in the force during motion could be due to acceleration or interaction of the magnet with the two bed regions, but the reasons have not been resolved further. Work input to the cryocooler could be computed provided the force and the position could be measured with a better resolution. The force on the displacer was not measured because a load cell suitable for the higher forces present at the increased mass flow rates was not available.

Figure 31, temperature difference along bed versus CHEX input power, shows the results of a bed thermal conduction measurement. The bed was equilibrated near $77 \mathrm{~K}$ by filling the magnet dewar with liquid nitrogen. Heat was input to the CHEX, and the resulting temperature difference across the bed was measured and plotted. A measurement at lower temperatures was planned but a power outage and subsequent apparatus failure prevented it. This information would be useful for evaluating the effects of thermal conduction along the bed on refrigerator performance. Future plans include repeating this measurement and analyzing the data.

Figure 32 shows various measured $\mathrm{GdNi}_{2}$ load curves at a $7 \mathrm{~T}$ magnetic field. The loads-were incremented from $0 \mathrm{~W}-1.5 \mathrm{~W}$, usually with small power increments. The HHEX temperature was between $76 \mathrm{~K}-86 \mathrm{~K}$, depending on the load curve. The helium mass flow rate was varied from $0.23 \mathrm{~g} / \mathrm{s}$ to $0.36 \mathrm{~g} / \mathrm{s}$. Cooling power is observed to increase with increase in mass flow rate. Measured cooling power ranged from $0.6 \mathrm{~W}-0.9 \mathrm{~W}$ at $40 \mathrm{~K}$. 


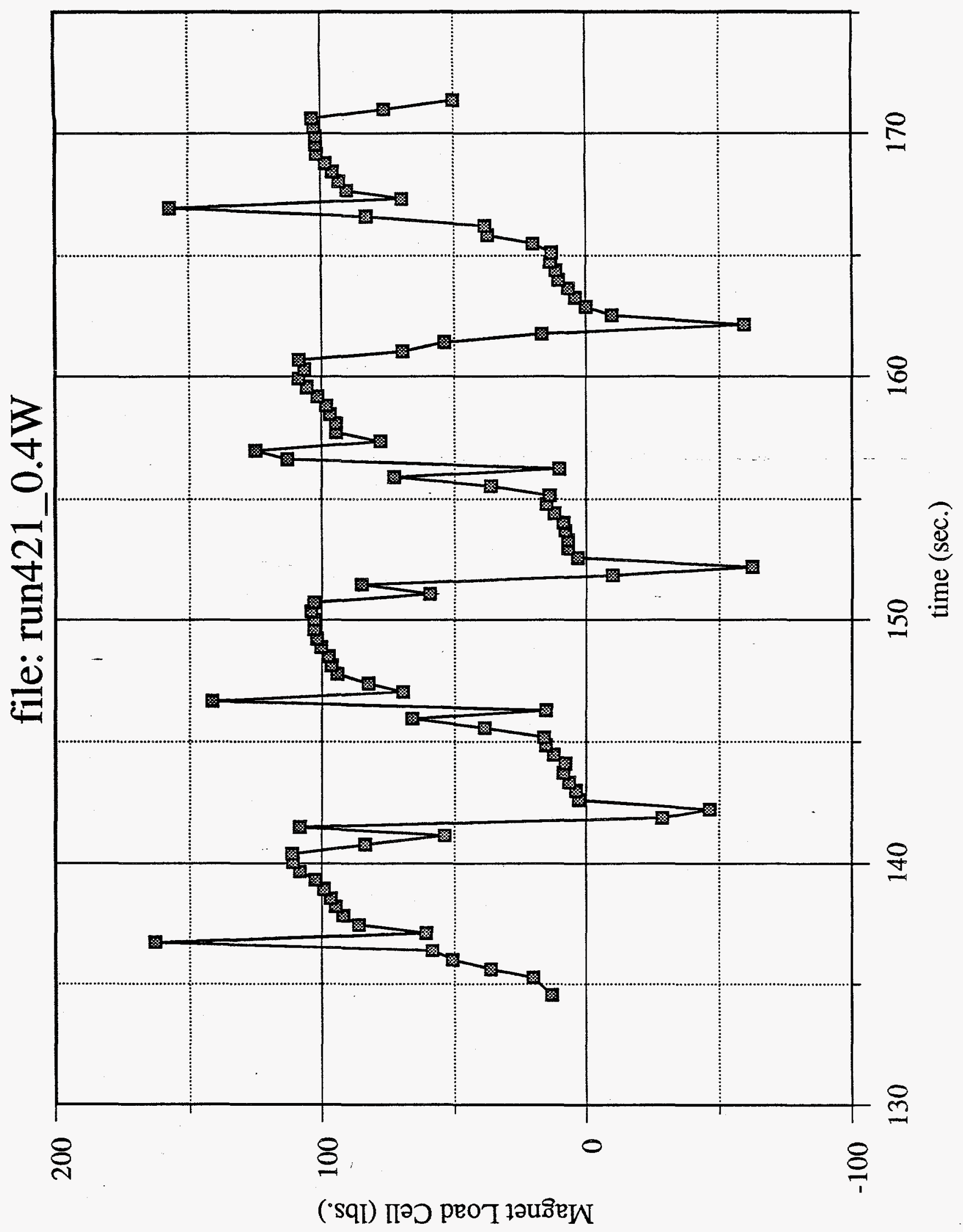

Figure 30. Magnet Load Cell Force vs. Time 


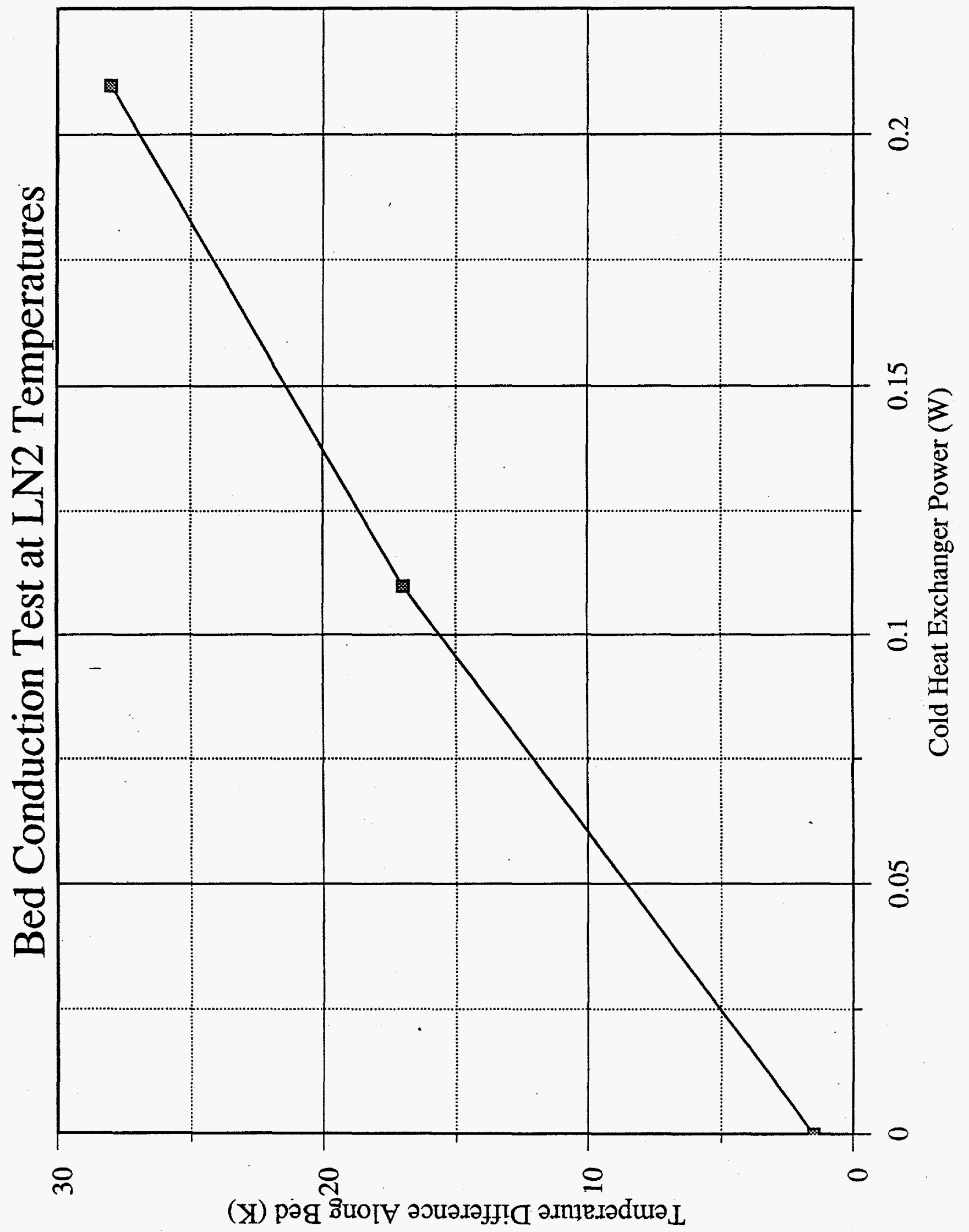

Figure 31. Temperature Difference Along Bed vs. CHEX Input Power Thermal Conduction Test 


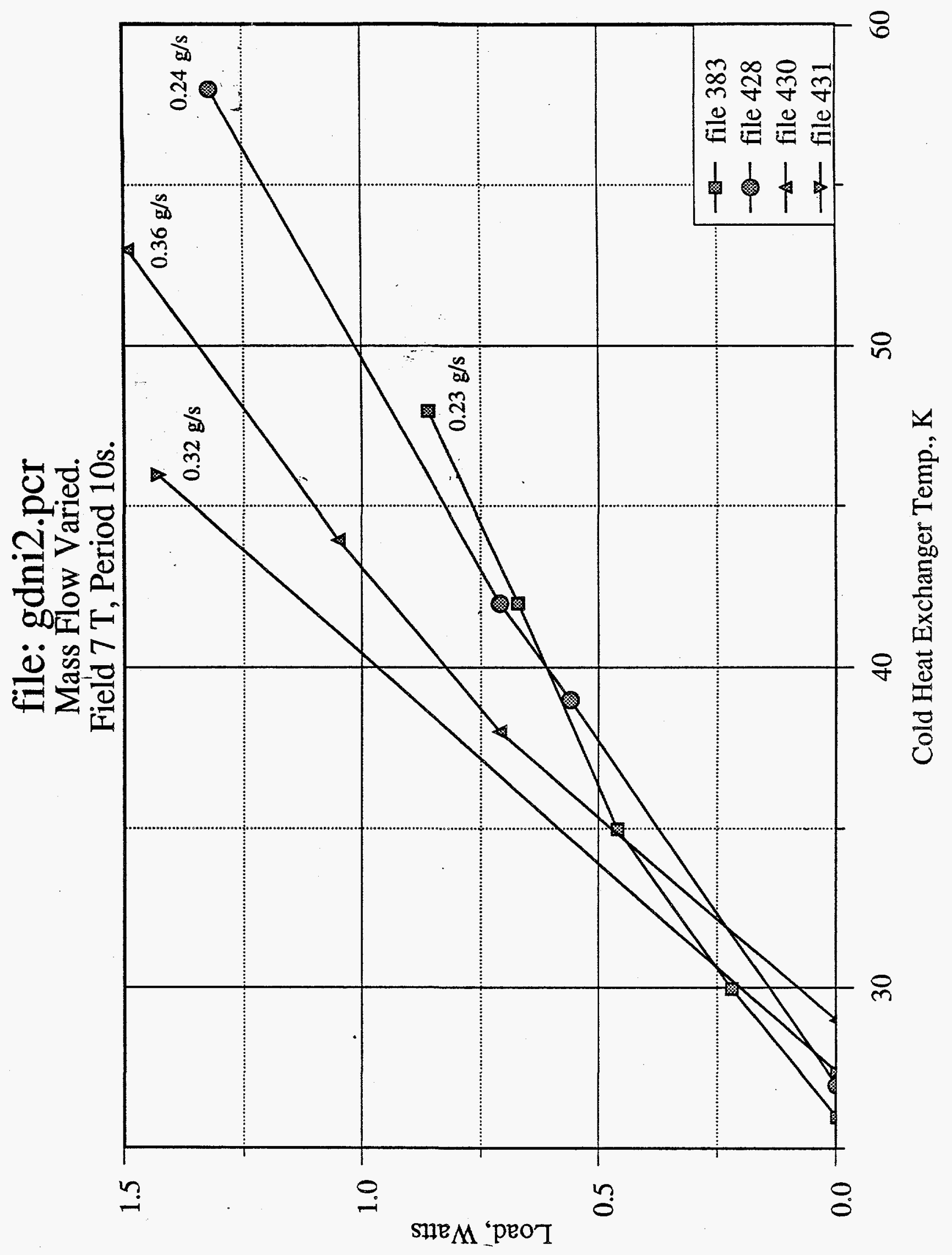

Figure 32. $\mathrm{GdNi}_{2}$ Load Curves at $7 \mathrm{~T}$ Magnetic Field 
The load curve of file 428, in Figure 32, shows a good indication of AMR performance at the desired temperature range of operation. The HHEX was at $77 \mathrm{~K}$ with a mass flow rate of $0.24 \mathrm{~g} / \mathrm{s}$ yielding a $0.6 \mathrm{~W}$ of cooling power at $40 \mathrm{~K}$ CHEX temperature. The displacer motion distance was $37 \mathrm{~mm}$ with $3.65 \mathrm{~atm}$ of helium pressure.

\subsubsection{Comparisons to the Model}

A time-dependent model of the AMR has been developed, based on the treatment of a passive regenerator in which the heat capacity of the pore fluid in the regenerator is assumed to be negligible compared to the solid. ${ }^{13}$ The magnetocaloric effect is added by assuming that the magnetic material undergoes adiabatic magnetization or demagnetization between fluid flow periods. The measured material properties are used, including the effect of hysteresis.

The heat transfer coefficient between the fluid and bed is obtained from empirical correlations for beds of packed particles. ${ }^{6}$ Particle sizes are between $0.001 \mathrm{~m}$ and $0.002 \mathrm{~m}$ for $\mathrm{GdNi}_{2}$, and between $0.002 \mathrm{~m}$ and $0.004 \mathrm{~m}$ for $\mathrm{Er}_{0.86} \mathrm{Gd}_{0.14} \mathrm{Al}_{2}$; particle shapes are irregular. Using the correlations, it is assumed that the particles are spheres with the radius of $0.0015 \mathrm{~m}$ for $\mathrm{GdNi}_{2}$, and $0.003 \mathrm{~m}$ for $\mathrm{Er}_{0.86} \mathrm{Gd}_{0.14} \mathrm{Al}_{2}$. The heat transfer between the bed and fluid is probably underestimated using this assumption.

The model accounts for the variation of properties with temperature of both helium and magnetic material. Axial thermal conduction through the bed is taken into account through an empirical expression. ${ }^{9,10}$ Pressure drop is computed by a modified Ergun equation. 8

\subsubsection{Interpretation of $\mathrm{Er}_{0.86} \mathrm{Gd}_{0.14} \mathrm{Al}_{2}$ Results}

Figure 33 shows computed temperature profiles over a complete AMR cycle. The sample AMR computation is of the following parameters: cold (hot) flow period, 0.5 $\mathrm{s}$; field change period, $4.5 \mathrm{~s}$; hot (cold) gas flow, $0.7 \mathrm{~g} / \mathrm{s}$; magnetic field, $1 \mathrm{~T}$; pressure, $1 \mathrm{~atm}$; hot (cold) temperature, $19.25 \mathrm{~K}(10.25 \mathrm{~K})$. The output parameters are: maximum pressure drop, $0.1 \mathrm{~atm}$; thermal conduction, $0.011 \mathrm{~W}$; axial dispersion, $0.022 \mathrm{~W}$; pump work, $0.46 \mathrm{~W}$; net cooling (heat rejection), $0.014 \mathrm{~W}(0.88 \mathrm{~W})$.

Temperature profiles over the four parts of the AMR cycle are shown in Figure 33. The graphs are ordered in time from left to right, top to bottom. Over the positive and negative flow periods, profiles are shown at equal time intervals. As shown in the upper left graph, the field increase raises the profile. During positive flow, the profile shifts downward, as shown in the upper right graph. The field decreases, then lowers the profile further, as shown in the lower left graph. During negative flow, the profile shifts upward, shown in the lower right graph, thus returning to the initial lower 
position shown in the upper left graph.

Figure 34 shows computed load curves at a $1 \mathrm{~T}$ field for different gas flow rates through the beds (mdot). The cold and hot flow periods are $0.5 \mathrm{~s}$. The field change periods are $4.5 \mathrm{~s}$, yielding a total cycle period of $10 \mathrm{~s}$. The average gas pressure is $1 \mathrm{~atm}$. The HHEX temperature is $19.25 \mathrm{~K}$. Two experimental points are shown for comparison. Based on the conditions of the displacer and beds, the gas flow for the experimental points is $0.59 \mathrm{~g} / \mathrm{s}$.

The model appears to under-predict the performance of the device by a small amount. It is interesting to note that, regardless of the gas flow, within reason, the no-load temperature predicted by the model is approximately $9.75 \mathrm{~K}$. This is about 1 $\mathrm{K}$ above the experimental value.

Results using $\mathrm{Er}_{0.86} \mathrm{Gd}_{0.14} \mathrm{Al}_{2}$ indicate the detailed model of the AMR is accurate to within about $10 \%$ in predicting the no-load temperature span of the refrigerator.

\subsubsection{Interpretation of $\mathrm{GdNi}_{2}$ Results}

Figure 35 shows computed temperature profiles over a complete AMR cycle. The sample AMR computation is of the following parameters: cold (hot) flow period, 2.5 $\mathrm{s}$; field change period, $2.5 \mathrm{~s}$; hot (cold) gas flow, $0.28 \mathrm{~g} / \mathrm{s}$; magnetic field, $7 \mathrm{~T}$; pressure, $4 \mathrm{~atm}$; hot (cold) temperature, $77 \mathrm{~K}(40 \mathrm{~K})$. The output parameters are: maximum pressure drop, $0.07201 \mathrm{~atm}$; thermal conduction, $0.03787 \mathrm{~W}$; axial dispersion, $0.04502 \mathrm{~W}$; pump work, $0.353 \mathrm{~W}$; net cooling (heat rejection), $0.745 \mathrm{~W}(2.047 \mathrm{~W}$ ).

Temperature profiles over the four parts of the AMR cycle are shown in Figure 35. The graphs are ordered in time from left to right, top to bottom. Over the positive and negative flow periods, profiles are shown at equal time intervals. As shown in the upper left graph, the field increase raises the profile. During positive flow, the profile shifts downward, as shown in the upper right graph. The field decreases, then lowers the profile further, as shown in the lower left graph. During negative flow, the profile shifts upward, shown in the lower right graph, thus returning to the initial lower position shown in the upper left graph. 
$4.5 \mathrm{sec}$ field increase

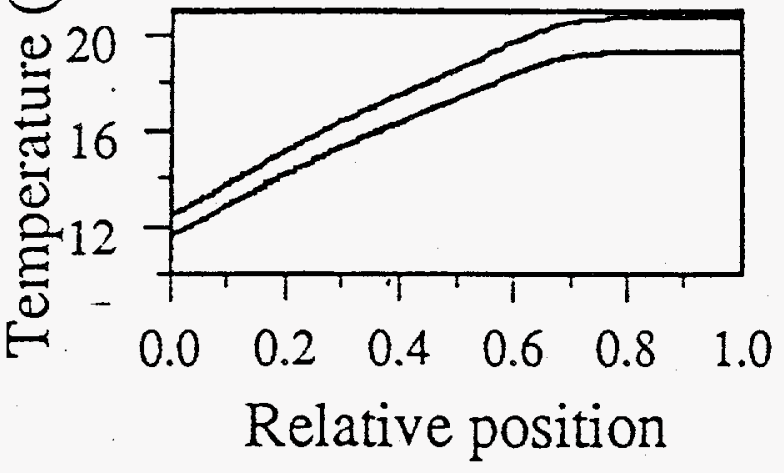

$4.5 \mathrm{sec}$ field decrease

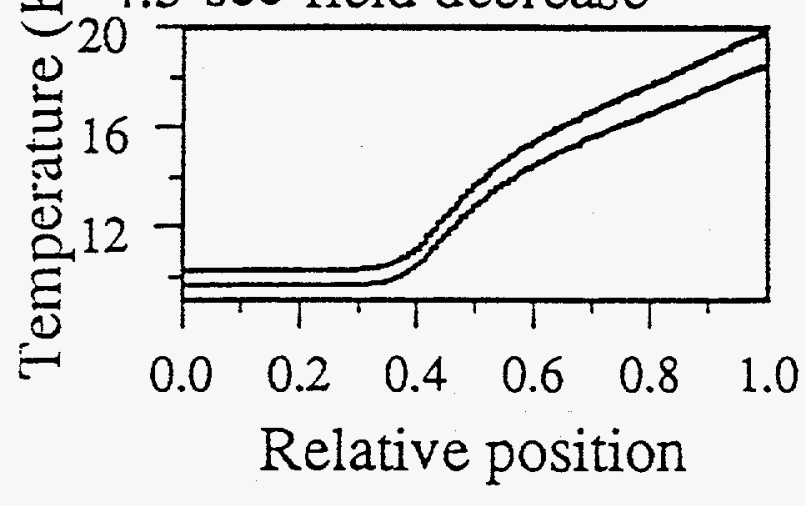

$0.5 \mathrm{sec}$ positive flow

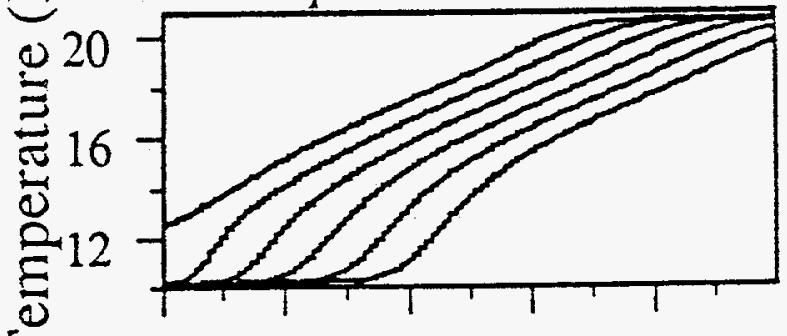

$\begin{array}{llllll}0.0 & 0.2 & 0.4 & 0.6 & 0.8 & 1.0\end{array}$ Relative position
$\widehat{C}_{20} 0.5 \mathrm{sec}$ negative flow

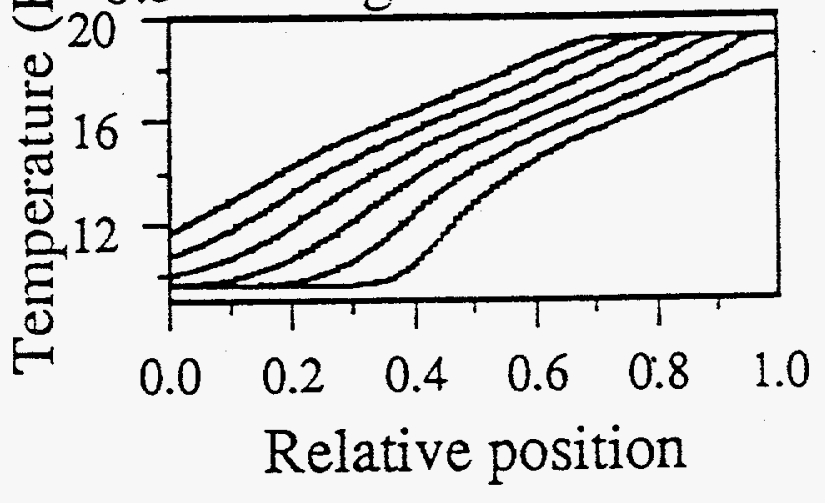

Figure 33. Computed Temperature Profiles over a Complete AMR Cycle for $\mathrm{Er}_{0.86} \mathrm{Gd}_{0.14} \mathrm{Al}_{2}$ 


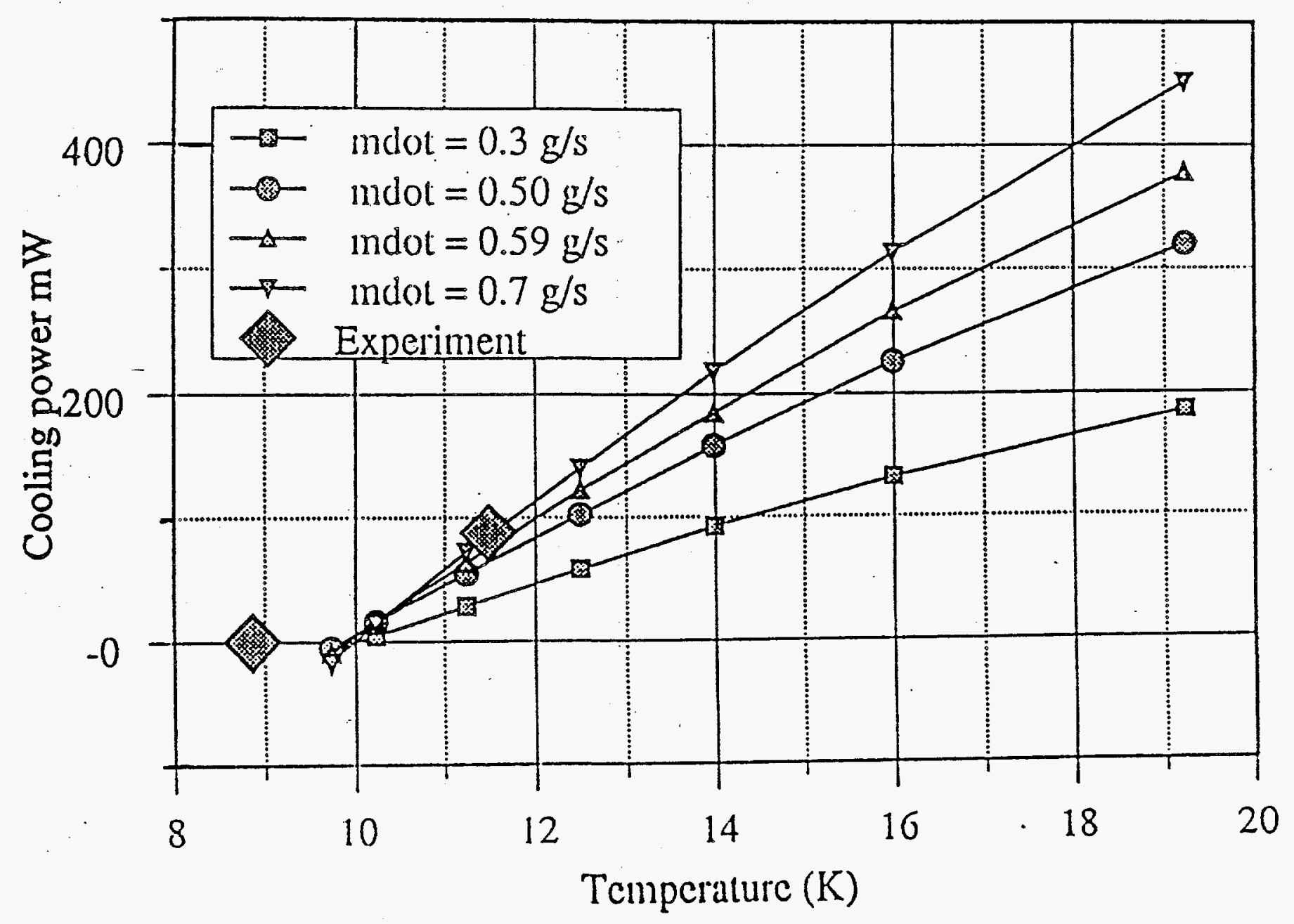

Figure 34. Computed $1 \mathrm{~T}$ Load Curves for Various Gas Flow Rates through $\mathrm{Er}_{0.86} \mathrm{Gd}_{0.14} \mathrm{Al}_{2}$ Beds 

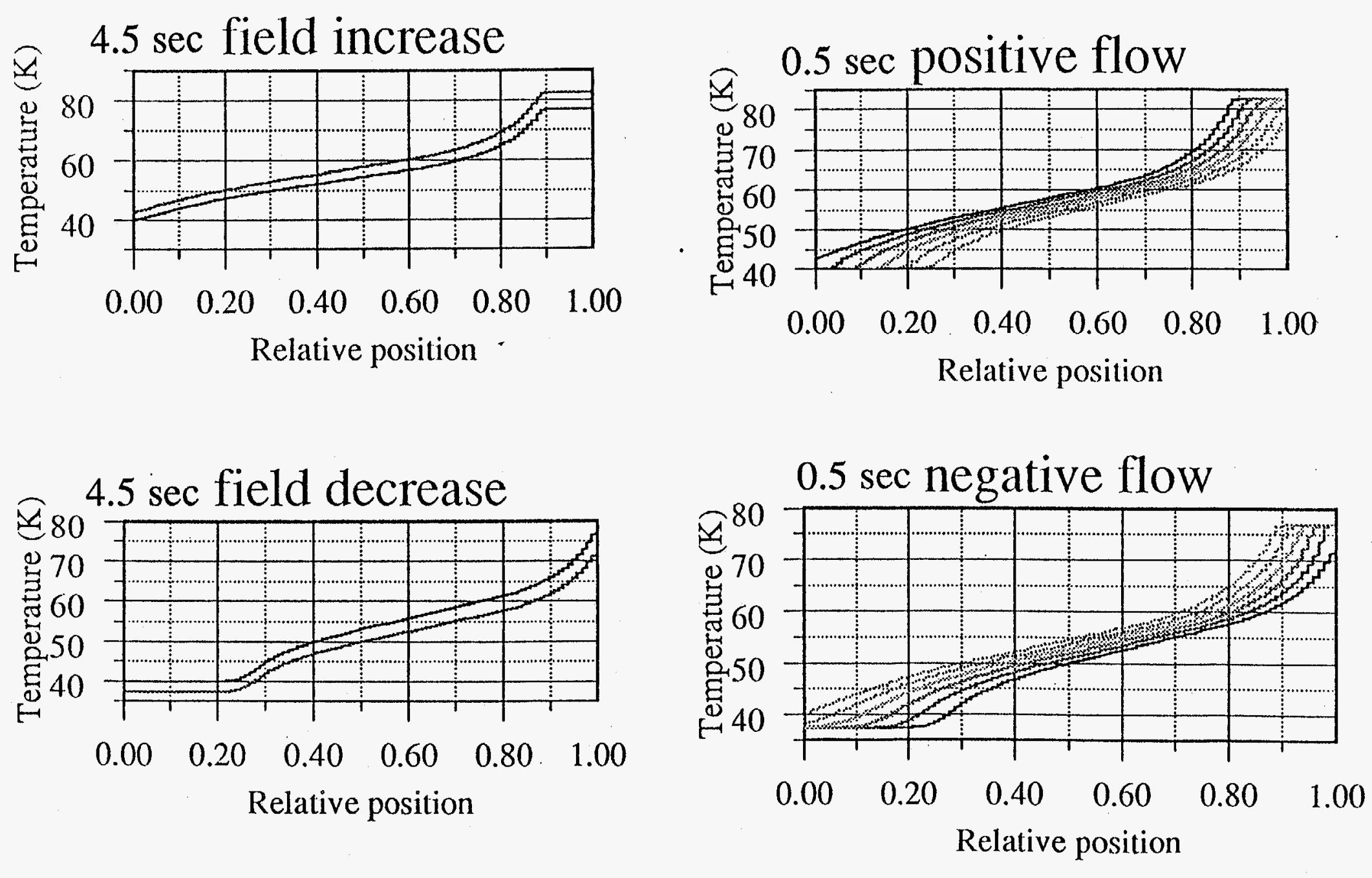
Figure 23 indicates that the material has a no-load temperature span from about $75 \mathrm{~K}$ to about $28 \mathrm{~K}$, at $7 \mathrm{~T}$. The model, which neglects parasitic heat leaks, predicts a no-load cold temperature about $10 \mathrm{~K}$ below that observed.

The experimental load data shown in Figure 32 was modeled using similar experimental conditions. A comparison between actual and modeled data was conducted. Figures 36 - 39 show the comparison between the modeled and experimental data for each run separately. As indicated by the plots of the measured and modeled load data, the results differ by $0.35 \mathrm{~W}-0.8 \mathrm{~W}$, depending on the particular load curve. The load curves were parallel in most cases, however, suggesting that the error margin is simply a constant factor, introduced by a systematic error in one of the experimental parameters.

After running the experiment for a few months, the $\mathrm{GdNi}_{2}$ magnetic material bed was found to be degraded. There was a significant volume loss (3-9\%) in the bed material because of an improper retaining screen size. This may have been the cause of the negative delta in load power between the experimental and modeled data.

To investigate this problem, the particle size distributions of the original bed material and the material removed from the degraded bed were measured. Figure 40 indicates the bed particle size distribution of the original material and the bed material. The majority of the distribution was unchanged. There was a decrease in the largest particle size population and an increase in the smallest size population. The impact of these findings on the actual results will be studied and a new bed with the appropriate retaining screen size will be made and retested. 


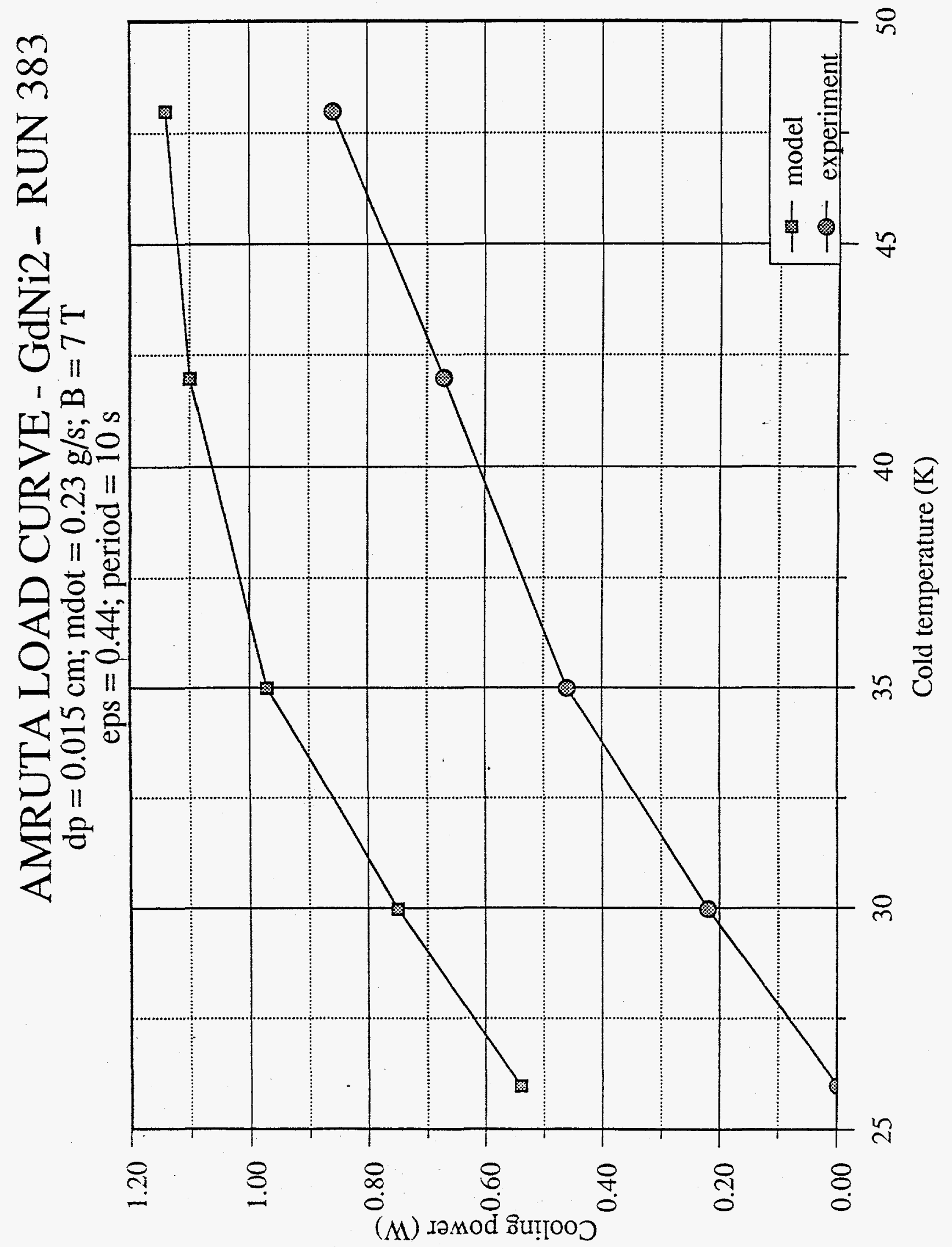

Figure 36. Comparison between the Modeled and Experimental Data of Run 383 


\section{AMRUTA LOAD CURVE - GdNi2 - RUN 428}

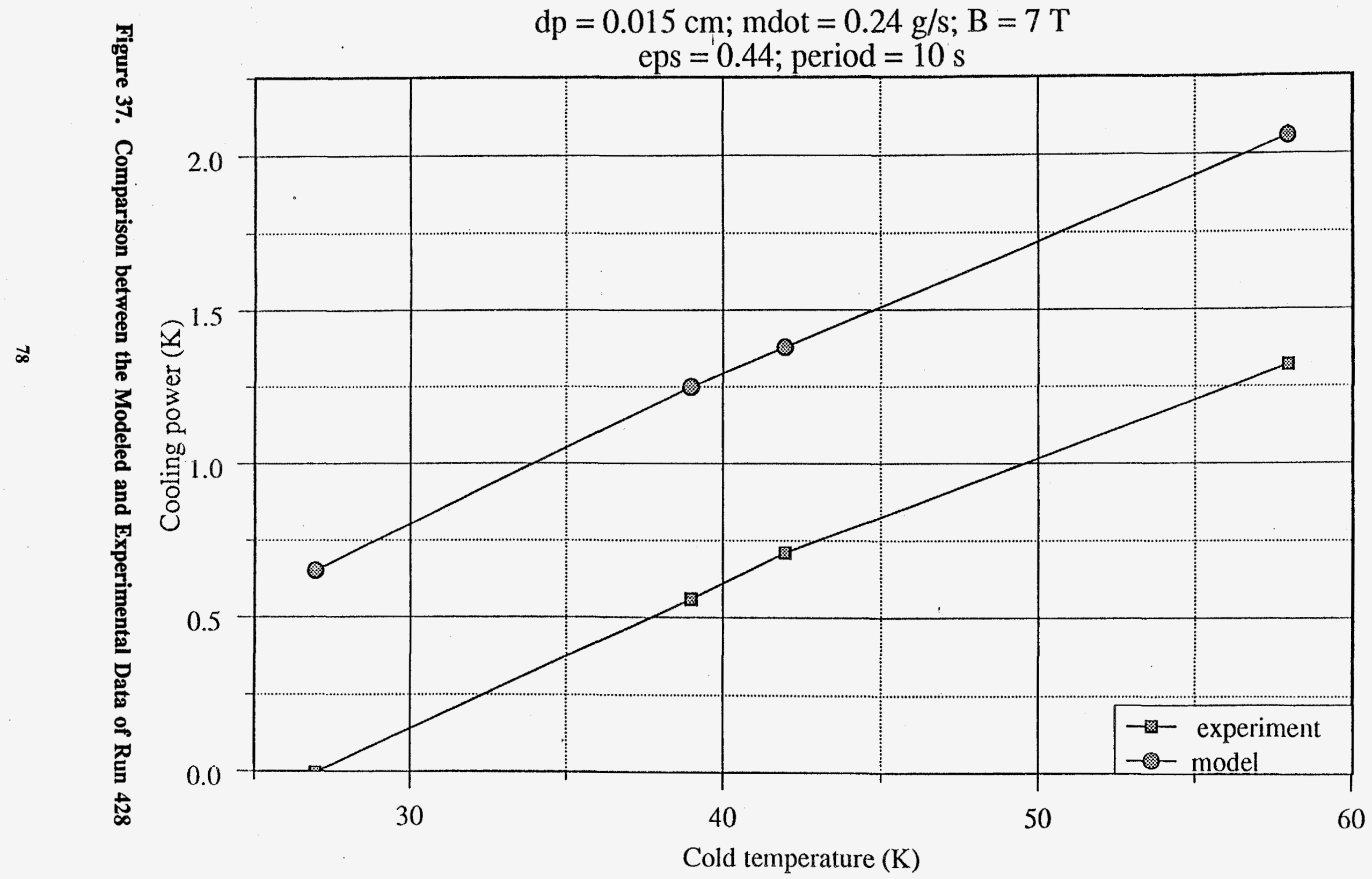




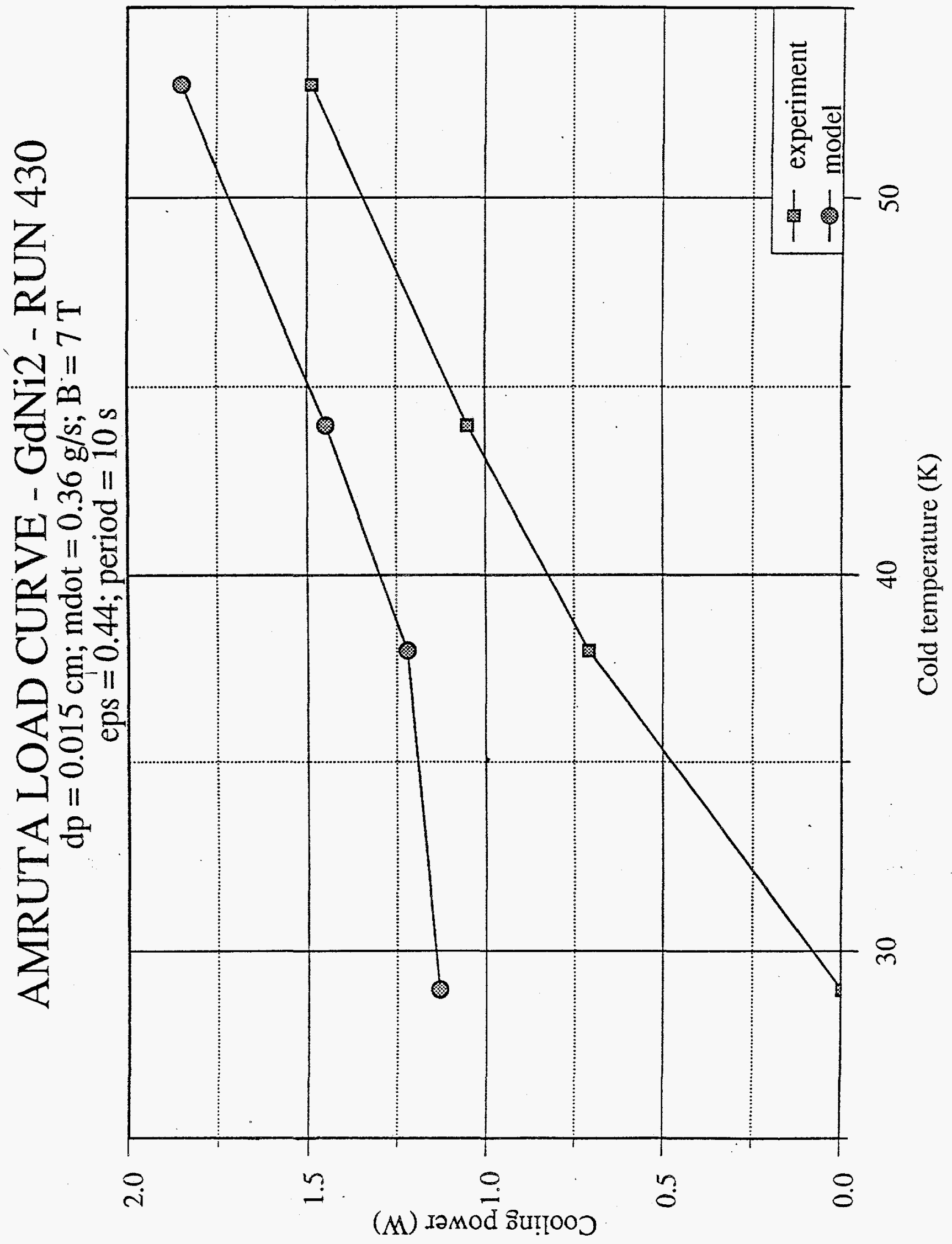

Figure 38. Comparison between the Modeled and Experimental Data of Run 430 


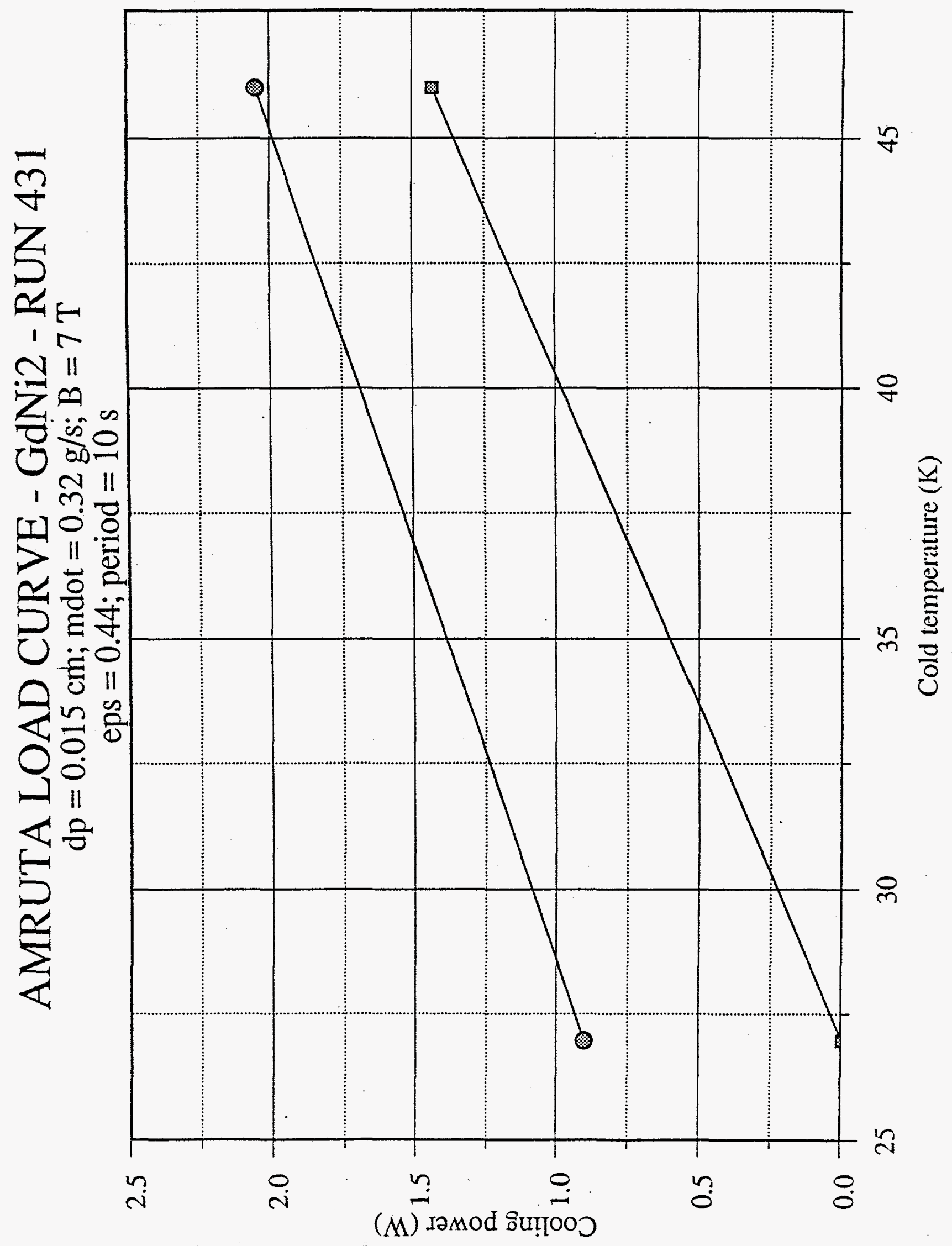

Figure 39. Comparison between the Modeled and Experimental Data of Run 431 


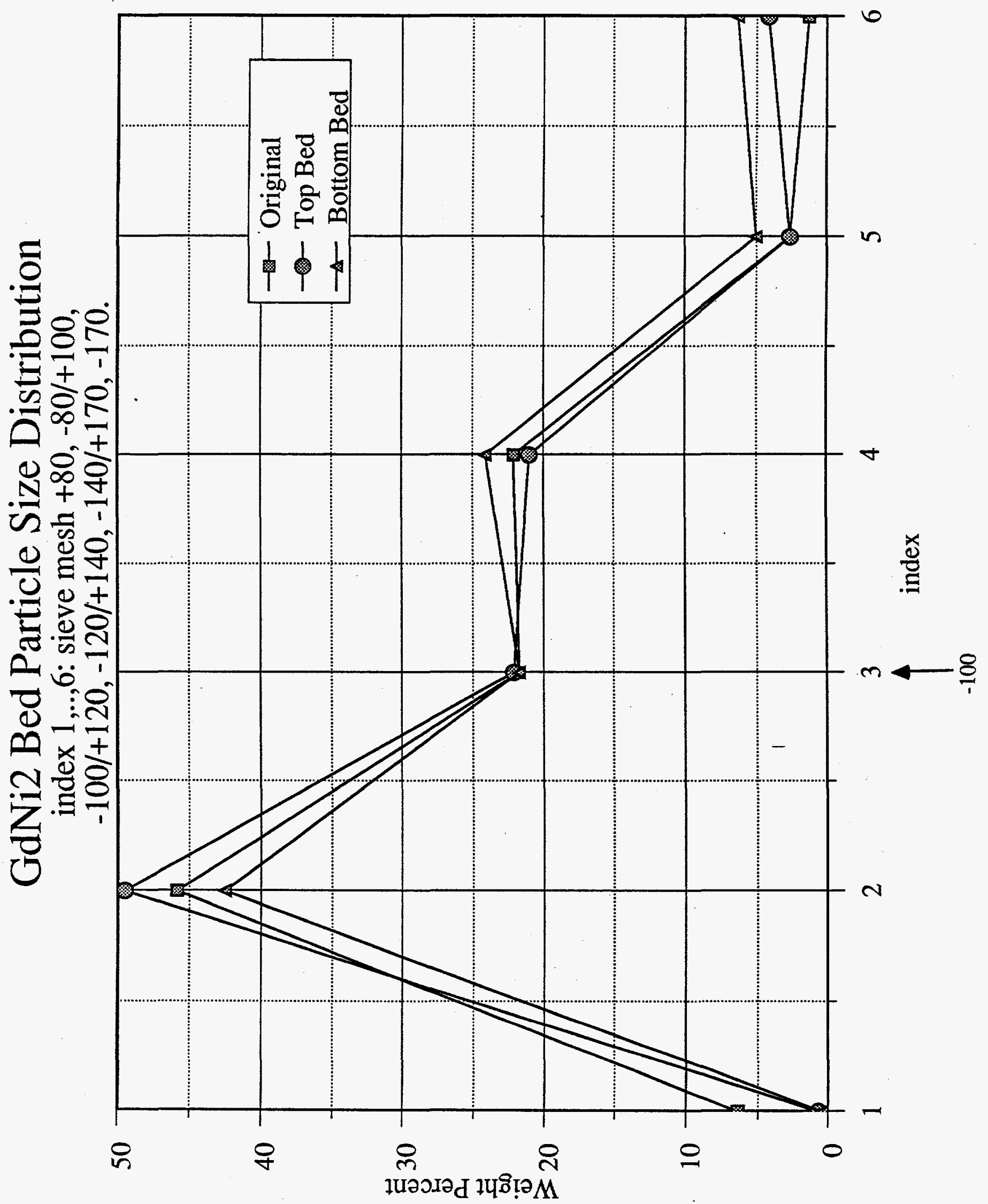

Figure 40. Bed Particle Size Distribution of the Original Material and the Bed Material 


\subsection{Testing Summary and Conclusion}

Testing of $\mathrm{Er}_{0.86} \mathrm{Gd}_{0.14} \mathrm{Al}_{2}$ in the AMR apparatus indicated that the detailed model of the AMR can predict to within about $10 \%$ the no-load temperature span of the refrigerator.

Testing of $\mathrm{GdNi}_{2}$ in the AMR apparatus produced important results. It was shown that the device can operate across large temperature spans (nearly $50 \mathrm{~K}$ in the no-load case). Substantial cooling load powers were achieved. Up to $0.9 \mathrm{~W}$ at $40 \mathrm{~K}$ with the HHEX temperature at $77 \mathrm{~K}$ were achieved. However, discrepancies were observed between predicted and measured performance. As mentioned earlier, the measured and modeled load data of the $\mathrm{GdNi}_{2}$ results differ by $0.35 \mathrm{~W}-0.8 \mathrm{~W}$, depending on the particular run. The fact that the load curves, of modeled versus measured data, were parallel, suggests that the error is a constant factor and could be a result of the defective bed; the bed lost 3-9\% of its material after an extended period of operation. This is believed to be the cause of the discrepancies. Future testing of a new reconstructed $\mathrm{GdNi}_{2}$ bed has been planned.

\subsection{Preliminary Rotary Configurations}

Rotary configurations offer many desirable features. Besides continuous operation, the dead volume associated with each of the numerous beds is small, which enhances volumetric efficiency. Continuous operation eliminates the need for reciprocating helium gas flows and ancillary flow distribution hardware. It also benefits from low helium flow rates relative to noncontinuous operation. Each bed executes two AMR cycles during each rotation with a simple, continuous, low-speed, hermetic drive. Drive torque ripple is very low because of the symmetrically balanced fields and uniformly distributed material. The drive and refrigerator as a whole require low precision. This simplicity extends to the beds, packaged in stainless steel sleeves like beds in the AMR test apparatus currently operating at the Technology Center (the AMR test apparatus is described in Section 4.1.2). All rotary designs use fixed superconducting magnets which may be recharged during refrigerator operation without a shutdown. Helium boil-off in fixed magnets can be replenished easily by a liquid helium storage dewar. With moving magnets, helium can be replenished easily from a fixed dewar connected to the moving magnet's containment by a flexible transfer line. Finally, the rotary designs are compact and scalable.

While individual component functionality of reciprocating and rotary AMRs is similar, their operation is unique. The rotary configurations operate continuously by sequentially introducing a magnetic field to a series of many small regenerator beds. As more beds of smaller size are used, a true continuous process is approached. The heat transfer fluid (helium) flows in a closed flow loop in one direction only. Bidirectional flow through the beds is accomplished with manifolding dedicated to each bed. 
The system flow loop is simple, delivering constant flow to the hydrogen heat exchanger without interruption. The magnetization/demagnetization of individual beds occurs as a result of the relative rotary motion between magnets and beds. Configurations may include rotating magnets or beds.

As noted, these configurations use fixed magnets with the beds positioned circumferentially around the perimeter of a rotating wheel. Top and bottom wheel plates with integral radial flow passages sandwich a circumferential bed ring to complete the wheel assembly. One of several proposed bed ring concepts is illustrated in Figure 41. Top and bottom wheel plates with flow manifolding are added in Figure 42. Wheel rotation successively positions each bed into and out of high field regions twice each rotation. Helium gas flow through the beds is synchronized with this magnetizationdemagnetization sequence by upper and lower rotary dynamic face seals located at the wheel hub. These face seals consist of floating, nonrotating disks mated to the top and bottom rotating wheel plate surfaces. Relative sliding motion occurs between the fixed dynamic face seals and the wheel plates during operation. The seals are coupled to two pair of helium inlet and outlet ports in the housing via the four bellows shown. These bellows allow positioning flexibility of the seals on the wheel. Seal axial float accommodates wheel alignment errors and load-induced defiections in the housing. The details of a single-stage rotary dynamic seal are illustrated in Figure 43. Each bed in the bed rim of the wheel has a corresponding inlet and outlet at the I.D. of the wheel assembly to interface with the face seals. The integral radial flow passages within the wheel assembly couple each bed with its respective inlet and outlet pair. As the wheel rotates each bed inlet and outlet pair sequentially communicates with one of the inlet or outlet ports in the face seals, thereby coupling to the housing ports and the external piping network. This internal porting geometry controls both flow direction and timing through each bed. The wheel, as previously discussed, is enclosed in a hermetic housing that also encapsulates the drive. A pair of symmetrically-located racetrack magnets straddle the housing to generate the fields necessary for bed magnetization twice each wheel revolution. A complete single-stage wheel/housing/magnet assembly is illustrated in Figure 44 with housing ports, through drive shaft, and beds exposed.

Investigations up to this point have not uncovered any prior work related to rotary dynamic cryogenic face seals for AMR application. Preliminary laboratory tests conducted at Astronautics with Rulan $J$ materials at $77 \mathrm{~K}$ have been inconclusive. Other materials under consideration include various low friction and wear films such as diamond on stainless steel. These films would be deposited on polished, flat stainless disks. Another option in addressing the sealing issue involves further conceptual design efforts to derive a rotary configuration without seals.

The primary distinction between rotary designs lies in the number of stages occupied within a single wheel/housing assembly. In the simplest case, each stage resides in its own wheel/housing assembly. The stages are then interconnected by piping and a hermetic drive shaft. Denser packaging is achieved by either reducing the stages 
required or combining all stages into a single wheel/housing assembly. Reprèsentative pictorial schematics of three rotary configurations investigated are illustrated in Figures 45, 46, and 47. Figure 45 depicts a three-stage, three-wheel ("33") design. Figures 46 and 47 depict three-stage, single-wheel ("31") and two-stage, single-wheel ("21") designs, respectively.

The wheel assembly becomes increasingly complex as more stages are integrated into a single housing. The simplest embodiment of this configuration incorporates a single ring of beds (Figure 41) into a single wheel (Figure 42) and housing (Figure 44) to make a single stage. Three such stages are coupled in Figure 45 . Conversely, the bed ring for the three-stage configuration in Figure 46 requires three concentric circles of beds rather than only one. Each ring of beds requires a different magnetic material.

The flow distribution and proportioning between stages accomplished by external piping in Figure 45 must instead occur within the flow manifolding integral to the wheel in Figure 46. This manifolding is complex and expensive. The most promising compromise of a rotary configuration is illustrated in Figure 47. Two bed rings are incorporated into a single wheel and housing. Internal flow manifolding in the wheel is simplified relative to the three-stage configuration while retaining a single housing.

The two-stage single-wheel design is the most feasible of these configurations. It offers the best compromise between simplicity, part count, and compactness. Further detailed analysis of rotary liquefier preliminary designs focused on the "21" configuration based on this assessment. This analysis addressed structural and thermal considerations and highlighted fundamental shortcomings of the configuration. 


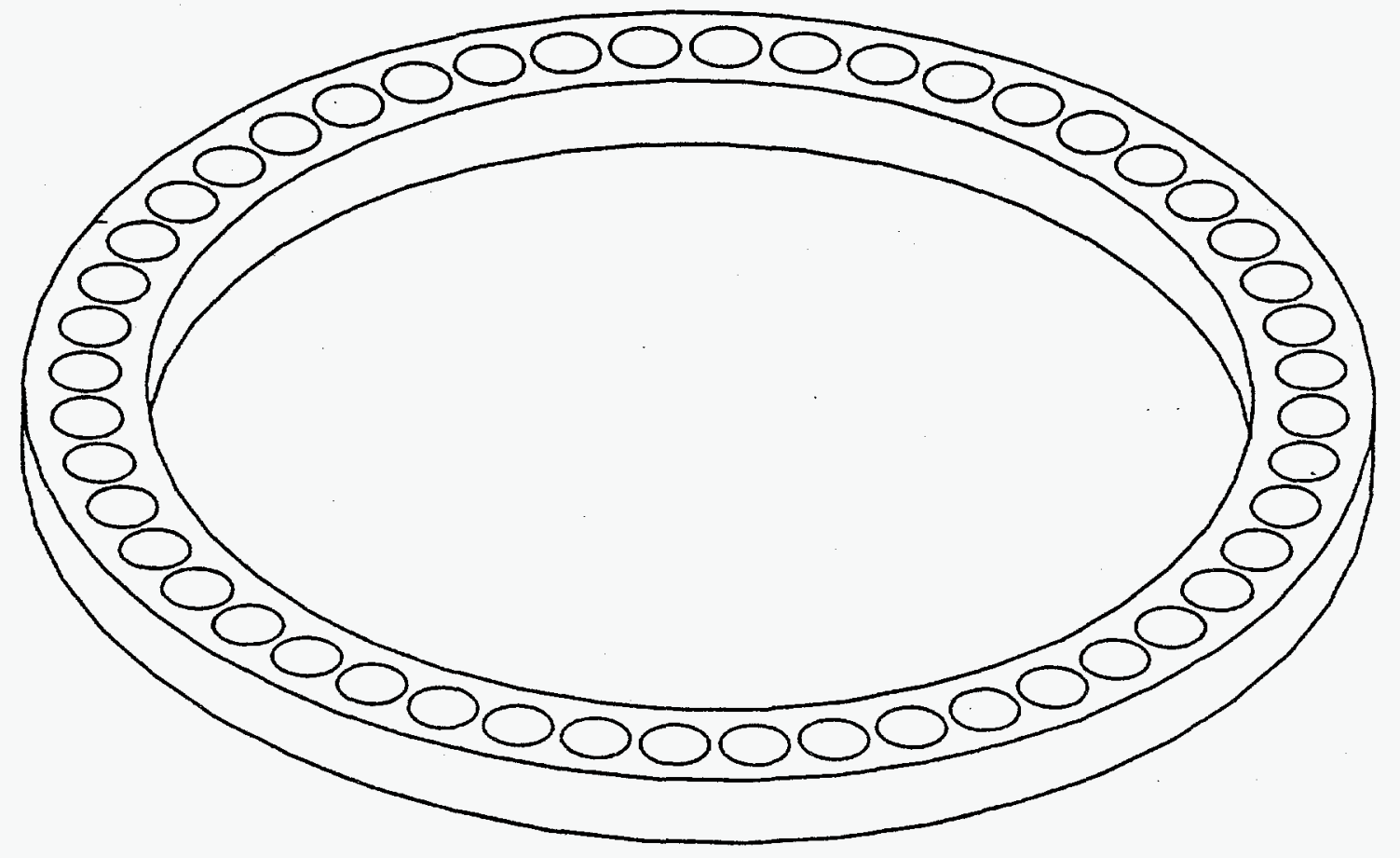

Figure 41. Rotary Configuration Bed Ring Concept 

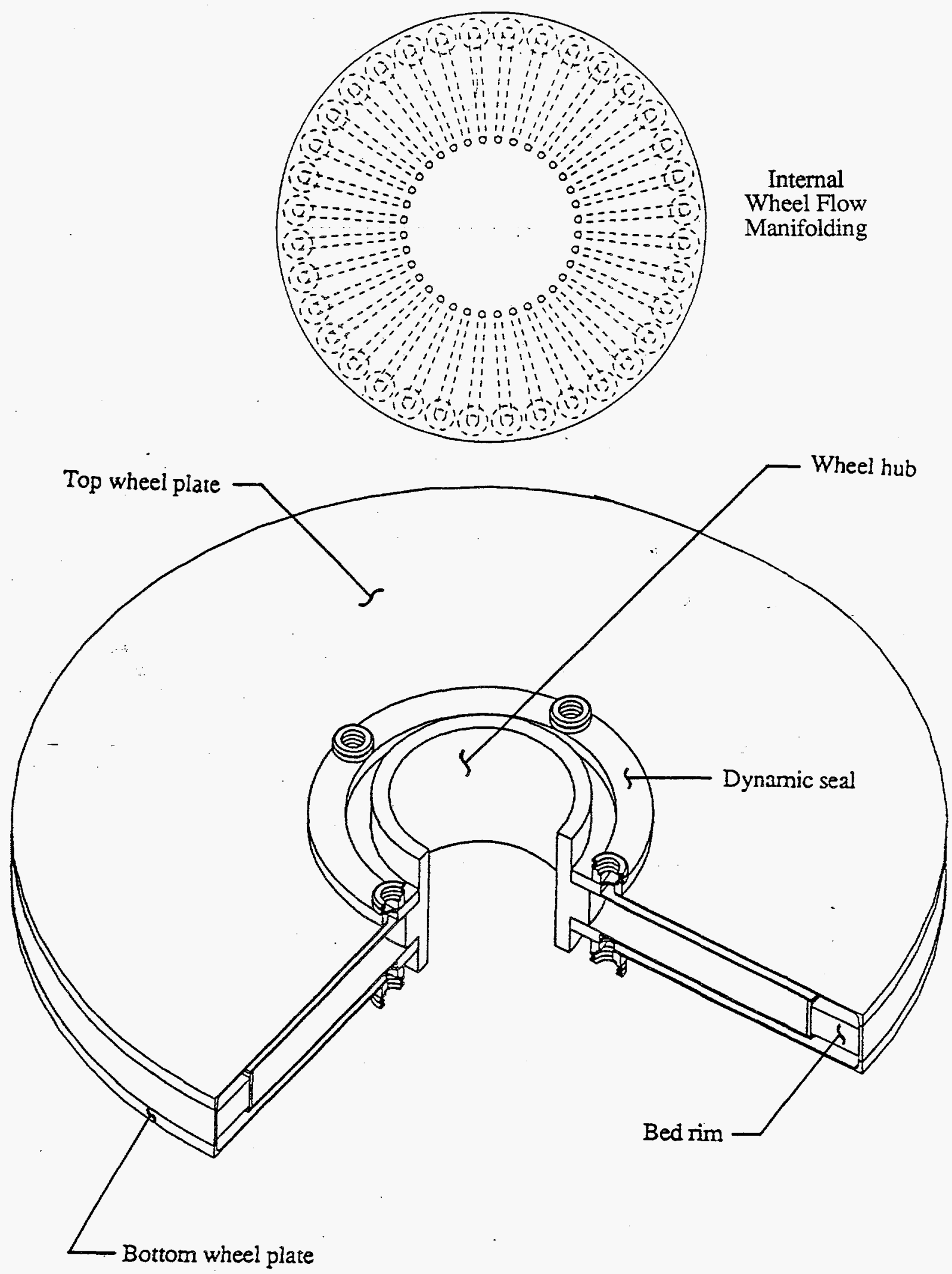

Figure 42. Rotary Configuration Wheel Assembly with Internal Flow Manifolding 


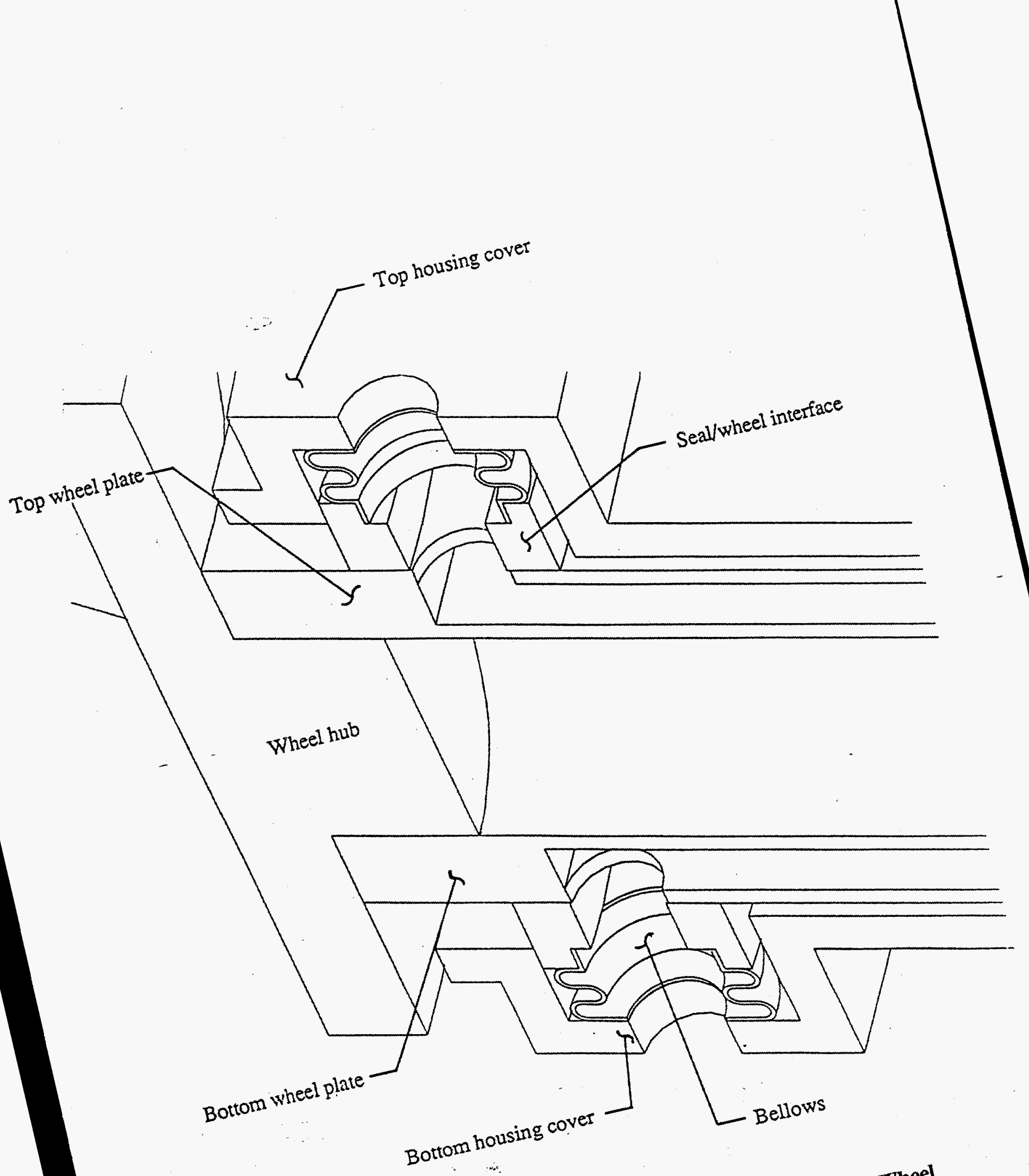




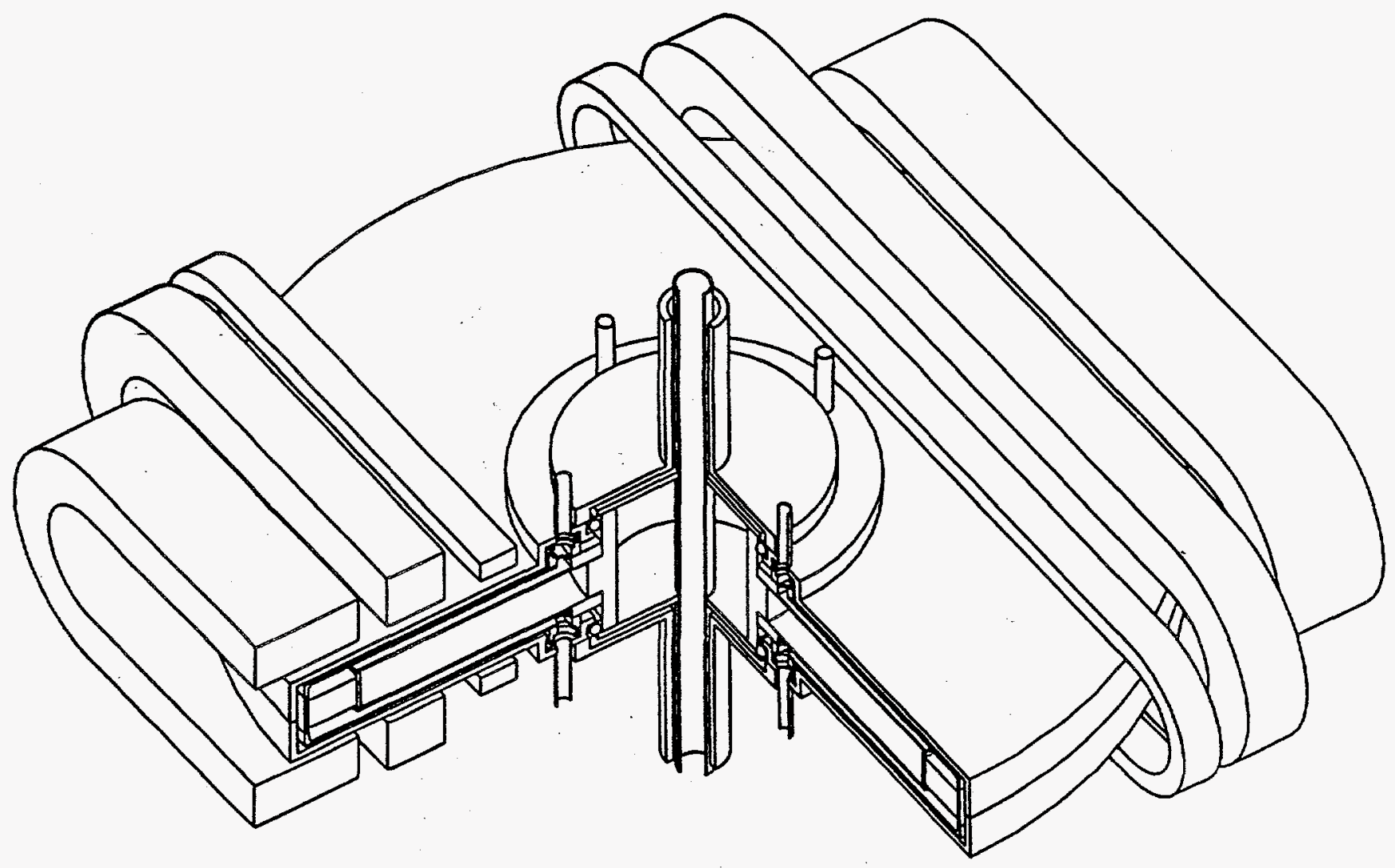

Figure 44. Single Rotary Liquefier Stage with Magnets, Housing, and Wheel 


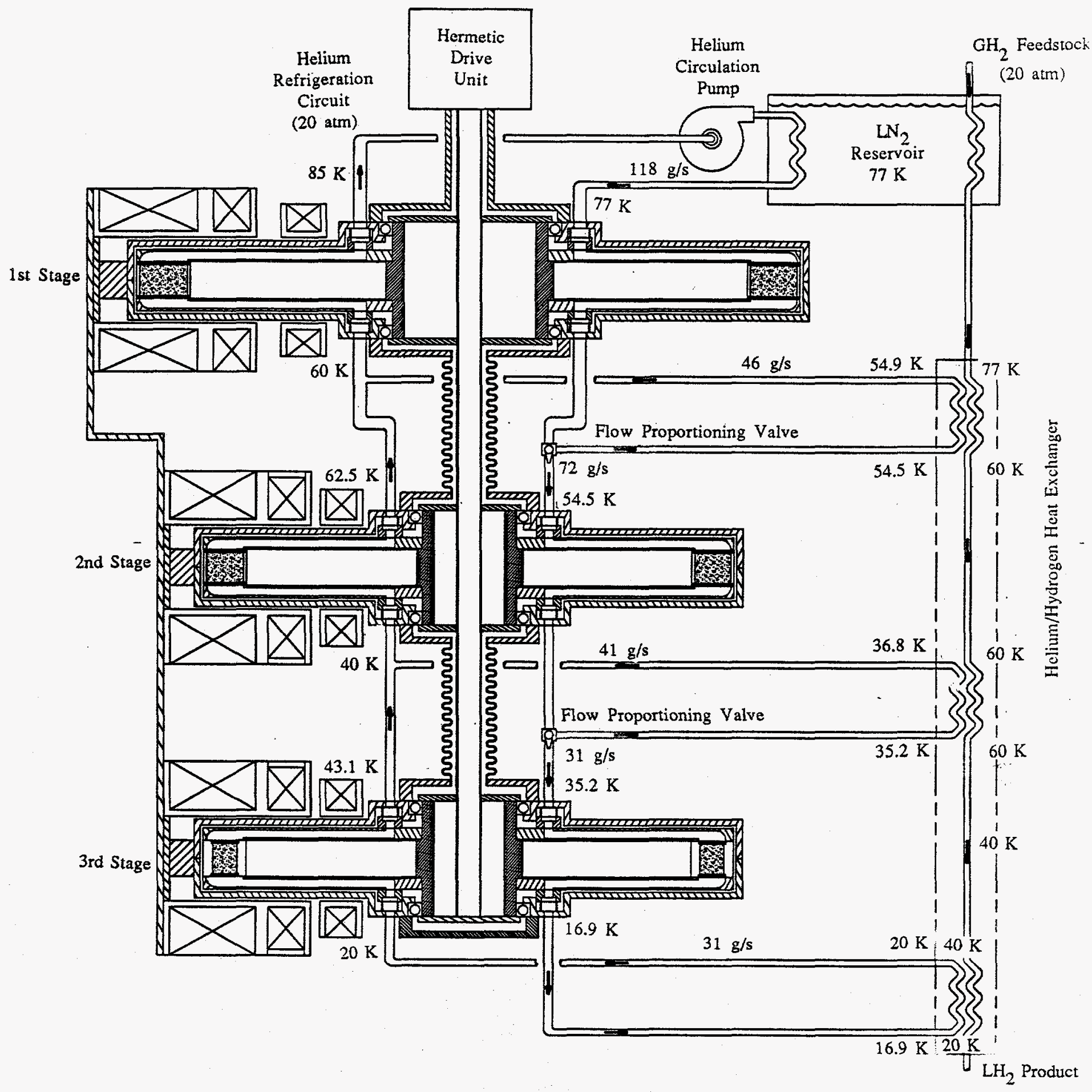

Figure 45. The Three-Stage "33" Rotary Liquefier Concept 


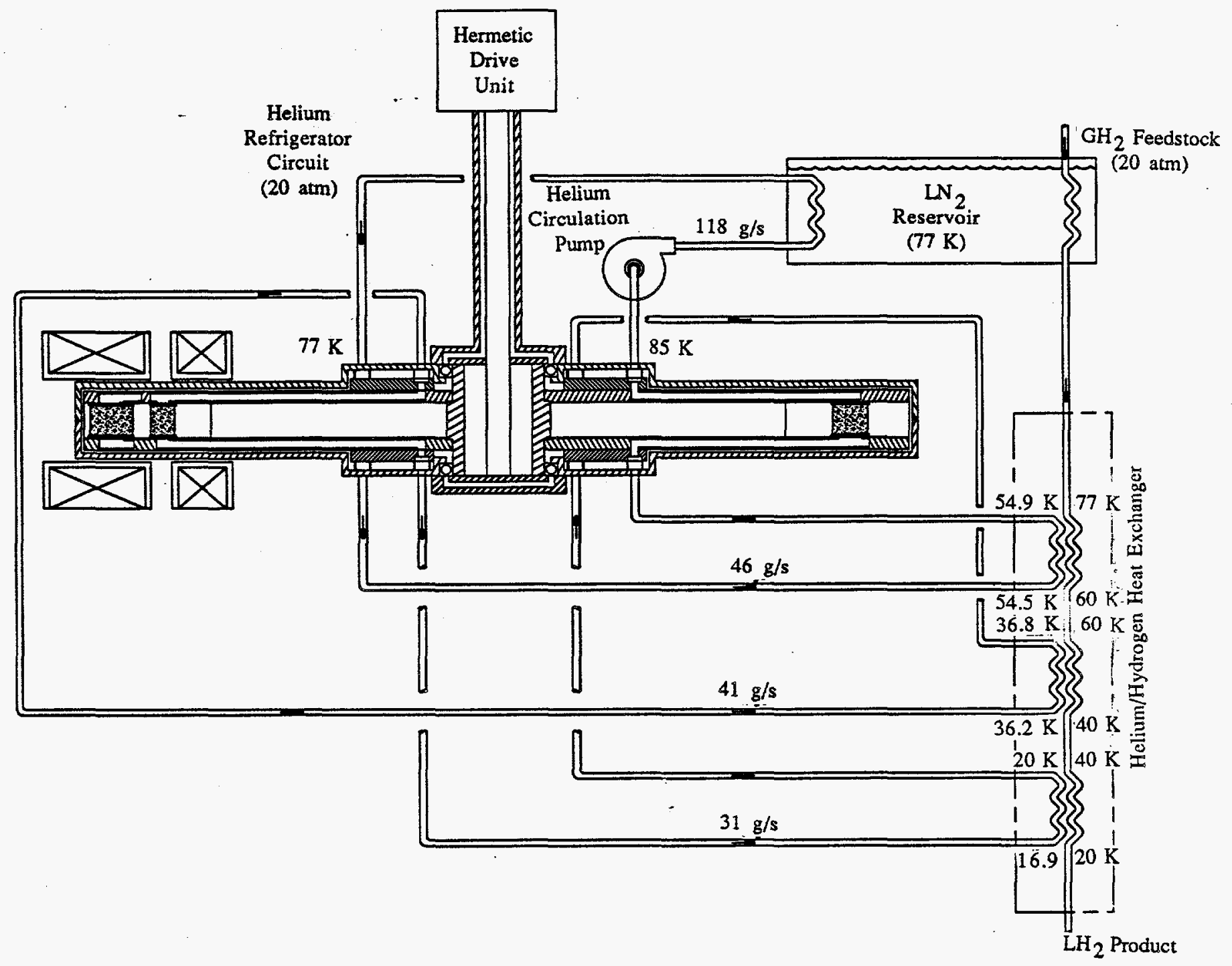

Figure 46. The Three-Stage "31" Rotary Liquefier Concept 

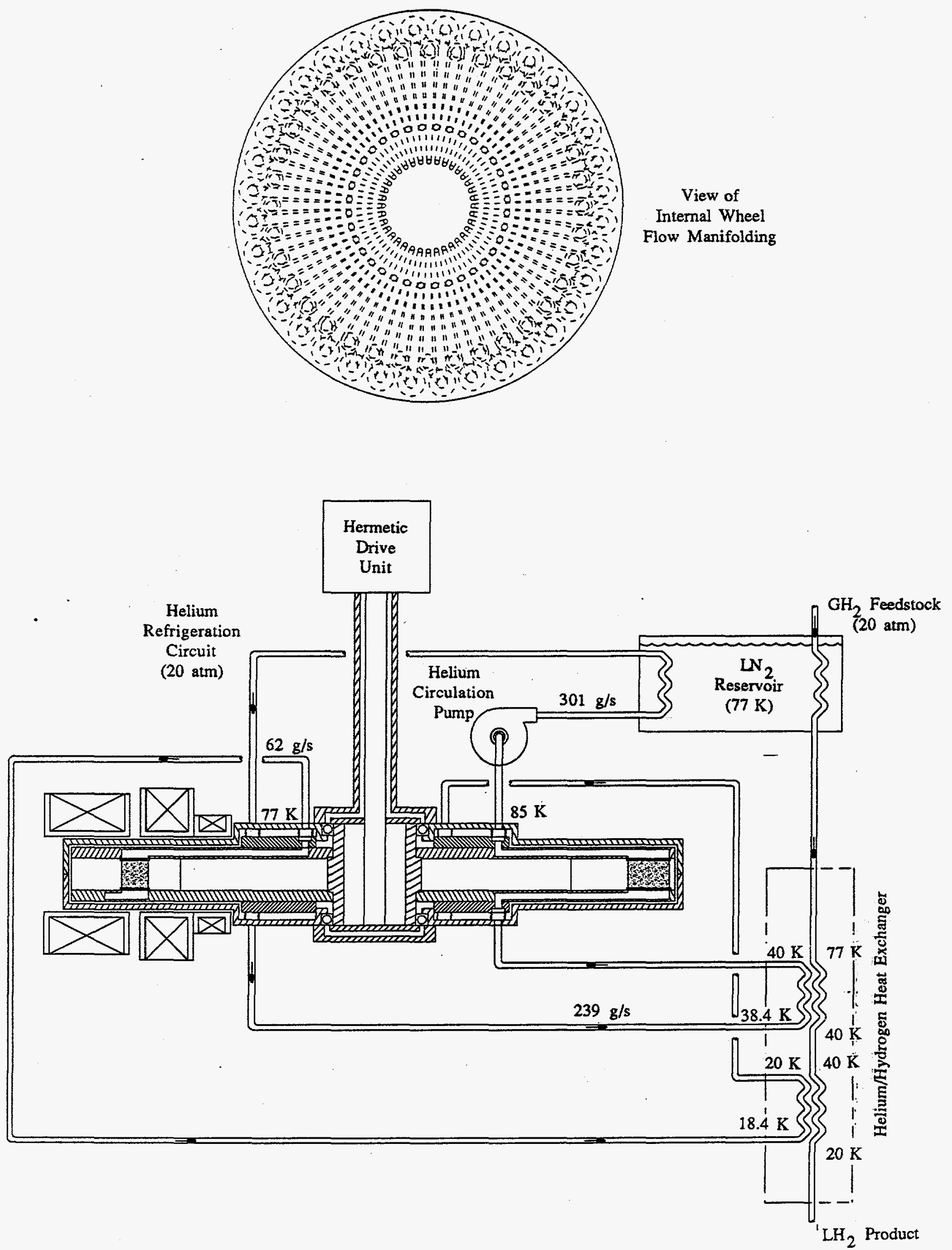

Figure 47. The Two-Stage "21" Rotary Liquefier Concept 
The large surface area of the rotary housing must sustain very large internal separating forces resulting from the $20 \mathrm{~atm}$ helium inside. The housing shape is very poor, in established pressure vessel design, and large distortions are possible. If parts of this load are shared by the bearing at the wheel hub, then bearing heating due to friction is generated. The total housing separation forces range between 450,000 to $500,000 \mathrm{lbs}$. Even with extremely high housing stiffness, it is difficult to achieve bearing loads below roughly $150,000 \mathrm{lbs}$. A bearing subjected to these loads, yet small enough to fit within the available space, is expected to fail after only a few hundred hours of operation. Furthermore, the heat generated by the bearing is expected to be about $400 \mathrm{~W}$. To avoid this undesirable feature, the housing could be externally supported to withstand the loading without significant deflection or bearing load. Structural modeling confirmed that a very massive housing would be required. However, the racetrack magnets occupy the space around the housing, preventing the placement of the reinforcement. The final design solution was a compromise, incorporating a moderate stiffness housing with axial bearing float to eliminate high bearing loads and heating. Housing deflections and bearing float were allowed to range between 0.01 and 0.02 inches.

In rotating designs, the parasitic heat sources investigated included eddy current heating and circumferential conduction in the wheel assembly, and dynamic seal blow-by resulting in by-pass helium flow around the regenerator beds. The total parasitic eddy current thermal load generated in the wheel during operation was estimated to be only $10 \mathrm{~W}$. A G-10 wheel design using thin wall stainless steel regenerator bed assemblies is located circumferentially around the wheel perimeter. -Circumferential conduction around the wheel between the hot and cold regions is estimated to be $12 \mathrm{~W}$. This wheel design integrates a G-10 structural backbone with individual radial flow tubes to direct the helium flow in the wheel from the seal at the ID to the beds at the OD. For comparison, if the wheel was fabricated as a sandwiched construction with 310 stainless steel wheel plates, with internal manifolding, as was first proposed, this heat leak would rise to about $1200 \mathrm{~W}$. This construction is also very complex and requires many joints and parts.

Because it is not realistic to expect a dynamic cryogenic seal to remain leak-tight, helium leakage into the housing cavity, and associated heat leaks will occur. The heat leak because of seal blow-by is proportional to the leakage flow volume. A simple flow model was generated to evaluate the sensitivity of liquefier performance to helium seal blow-by. This model includes the estimated seal-to-housing cavity blow-by. The model confirmed that the bulk of the helium leaking into the housing cavity originates at the $77 \mathrm{~K}$ inlet port. This helium leaks into the housing cavity where it mixes with other leaked helium from the first and second stages. The model predicts the equilibrium temperature of the helium within the cavity to be about $67 \mathrm{~K}$. This gas reenters the flow stream through seals at the downstream, lower-pressure seal return ports. The largest part of the total leakage occurs between the $77 \mathrm{~K}$ inlet and return ports. A small fraction of gas in the housing cavity at $67 \mathrm{~K}$ recombines with the flow streams between the first and second stages. 
Based on the model assumptions, $0.8 \%$ of the second stage flow will leak from 67 to $20 \mathrm{~K}$, resulting in a $242 \mathrm{~W}$ heat leak. Roughly $2.4 \%$ of the first stage flow will leak from $67 \mathrm{~K}$ to $40 \mathrm{~K}$, adding an additional $405 \mathrm{~W}$ heat leak. These small leak rates absorb almost one-half of total liquefier cooling power. Very high reliability, low leakage dynamic seals are required for acceptable liquefier performance.

\subsection{Preliminary Reciprocating Configurations}

Few features or characteristics are shared between the rotary and linear reciprocating configurations. While the linear reciprocating configurations do not generate continuous cooling as with rotary designs, they are more conservative, are lower risk, and offer their own set of desirable features. They are conservative because of our experience with such designs. Specifically, a small-scale reciprocating magnet AMR has been demonstrated at the Astronautics Technology Center. Reciprocating drives can be tailored to generate different dwell and travel times over a single period. This allows for sensitivity investigations into dwell and travel time proportioning within a cycle. This flexibility comes without a requirement for high precision in the drive components, or any other major components. The reciprocating configurations thermally and structurally decouple the multiple stages from one another so that heat leaks are minimal. Finally, the reciprocating designs can accommodate modular construction which can be appealing in scale-up to a 1 ton/day liquefier.

The reciprocating configurations are characterized by either moving magnets or beds coordinated with controlled, bidirectional helium flow to accomplish heat exchange with the hydrogen process stream. Helium flow through the beds occurs only when they experience a fixed high or low magnetic field. During the time when the fields on the beds are changing (i.e., magnets and beds in relative motion) helium flow ceases. This flow resumes once the fields on the beds stabilize (i.e., no relative motion between beds and magnets). A more detailed discussion of the operating sequence of a reciprocating AMR is presented in Section 5.7.

Evaluation of several reciprocating configurations quickly lead to selection of a moving magnet design. The moving bed designs require a way stationary piping can be coupled to reciprocating beds. Either dynamic sliding seals or long travel bellows are required for this coupling. The seals are undesirable for many of the same reasons described in the discussion of the rotary configurations. Unlike the "21" rotary design, interstage leakage is not possible with reciprocating configurations due to physical separation of the stages. Leaks in a reciprocating design would either by-pass individual beds or escape to the vacuum space. Long travel welded bellows, used in place of sliding seals, eliminate the leakage issue until the bellows fail. Long travel high differential pressure bellows require many convolutions, all subject to fatigue. Life tests of small short bellows have been promising but do not ensure long life in this high differential pressure application. As a result of these shortcomings, moving bed reciprocating configurations were not pursued. 
A second issue important to reciprocating configurations is the number of magnets and bed pairs in each stage. Thermodynamic modeling determines the total volume of bed material required for each stage, but not its distribution. Evaluation of the number of beds and magnets required is ultimately based on costs involved and performance required. In the reciprocating magnet design, the comparison is between stages consisting of two beds and a single magnet and a four-bed, two-magnet configuration. A four-bed per stage arrangement offers continuous cooling flow for heat exchange with the hydrogen stream but adds complexity and cost to the piping. Flow delivery of the helium circulation pump is half that of a two-bed per stage arrangement. Four beds per stage offer some redundancy if one of the two magnets in the stage fails. In this case, the second magnet could continue to operate on the other bed pair in the stage. These advantages do not come without cost. The added complexity increases the likelihood of a failure. Also, the total winding volume for two smaller bore magnets used in the four-bed arrangement is significantly larger than the volume in a larger bore single magnet operating on two beds in a stage. The two magnets per stage arrangement requires $50 \%$ more winding volume than with a single magnet per stage. Details of preliminary magnet winding volumes are given in Figure 48. (In this figure, magnet designation refers to individual preliminary magnet designs. The designation code indicates both the stage and number of beds in the configuration. The figure summarizes comparisons between first stage four-bed (14) and first stage two-bed (12) as well as second stage four-bed (24) and second stage two-bed (22) magnet designs.) Although the magnets referenced in the figure are not the same ones used in the final prototype detailed design, this same general relationship applies to them as well. When all issues were considered, a single moving magnet shuttling between two beds (as illustrated in Figure 49) was chosen for the preliminary design of each reciprocating configuration stage.

The piping system joins with each of the two beds illustrated in Figure 50. Unlike the rotary design, bidirectional pipe flow is characteristic of reciprocating configurations. Dead volumes become an important efficiency-reducing factor in these configurations. Flow rectification can be used to minimize their impact. The preliminary reciprocating flow schematic, incorporating flow rectification, is illustrated in Figure 50. Four directional flow valves are placed near the helium/hydrogen heat exchanger to generate intermittent, one-directional flow through the four parallel helium passages. This adds complexity and additional failure modes to the liquefier but does effectively eliminate the losses associated with dead volumes. 


\begin{tabular}{|c|c|c|c|c|c|c|c|c|}
\hline $\begin{array}{c}\text { Magnet } \\
\text { Designation }\end{array}$ & $\begin{array}{c}\text { Iron } \\
\text { Height } \\
(\mathbf{c m})\end{array}$ & $\begin{array}{c}\text { Iron } \\
\text { OD } \\
(\mathrm{cm})\end{array}$ & $\begin{array}{c}\text { Iron } \\
\text { Volume } \\
(\mathbf{c c})\end{array}$ & $\begin{array}{c}\text { Winding } \\
\text { ID } \\
(\mathrm{cm})\end{array}$ & $\begin{array}{c}\text { Winding } \\
\text { Volume } \\
(\mathbf{c c})\end{array}$ & $\begin{array}{c}\text { Separation } \\
\text { Dist. } \\
(\mathrm{cm})\end{array}$ & $\begin{array}{c}\text { Magnet } \\
\text { WT. } \\
(\#)\end{array}$ & $\begin{array}{c}\text { Total } \\
\text { Winding } \\
\text { Volume } \\
(\mathbf{c c})\end{array}$ \\
\hline 14 & 53.4 & 50.0 & 80800 & 16.8 & 8095 & 43.0 & 1500 & 16190 \\
\hline 12 & 60.0 & 55.0 & 108100 & 19.4 & 11170 & 54.5 & 2000 & 11170 \\
\hline 24 & 39.0 & 30.0 & 18091 & 11.8 & 3454 & 35.0 & 360 & 6908 \\
\hline
\end{tabular}

Magnet Designation Code:

$\mathrm{X} \times \quad$ 1st number indicates stage number

$\mathrm{X} X$ 2nd. number indicates \# of bed assemblies in stage

Figure 48. Factors for the Comparative Evaluation of 1st\&2nd Stage Reciprocating Magnets 


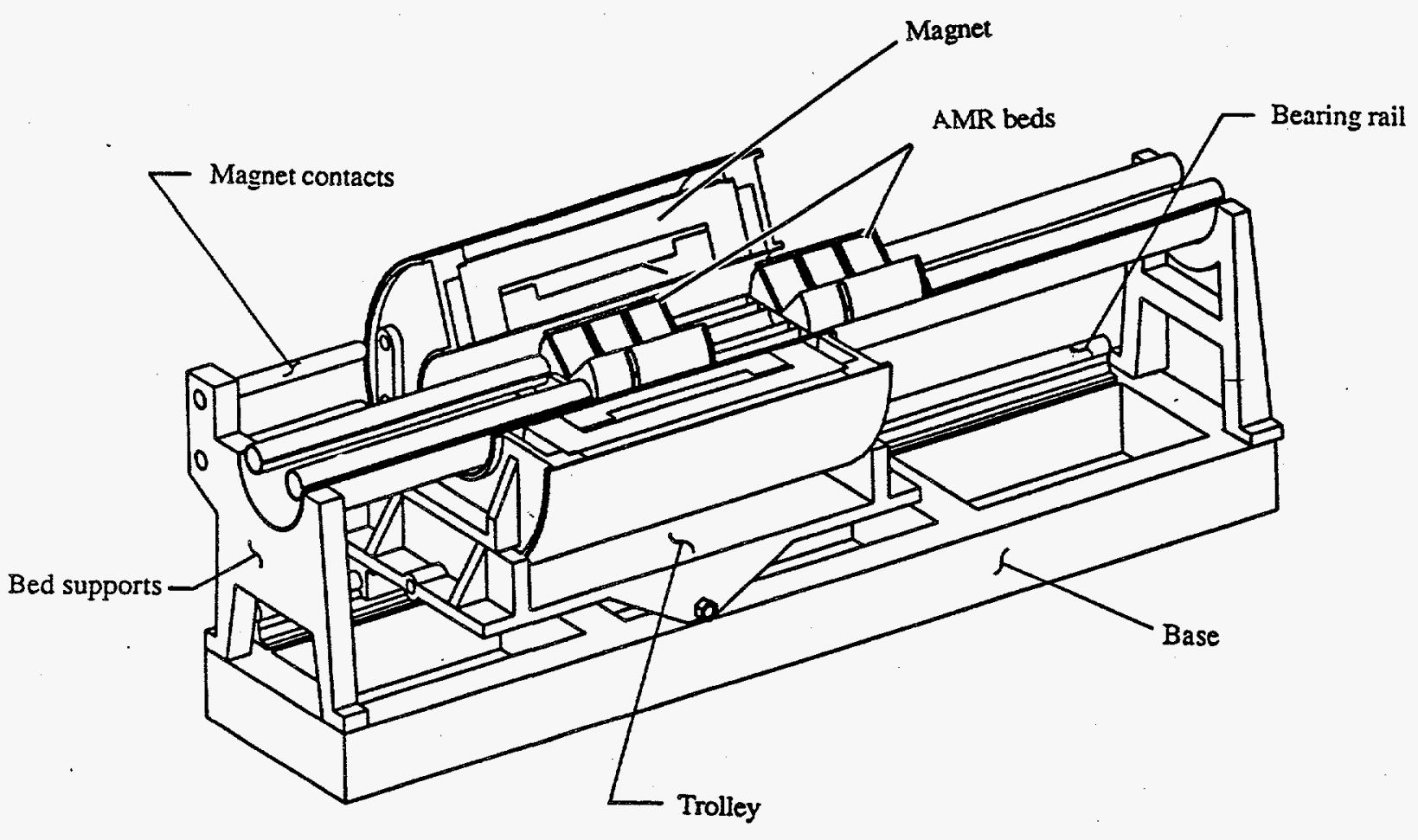

Figure 49. AMR Trolley Structure, Preliminary Design of a Reciprocating Stage 
Magnet motion, like pipe flow, is also bidirectional. Moving magnets pose several additional structural, thermal, and electrical design considerations not posed by fixed magnets. The magnets must be supported by bearings operating in the vacuum environment. These bearings generate heat and require lubrication. Typically, dry lubricants provide adequate lubrication to keep heating to a minimum with no sacrifice on bearing life. The magnets must be insulated from any heating that does occur to minimize helium boil-off. Because the magnets reciprocate, tension straps, which could reduce bearing loads, cannot be effectively used for support without adding significant structural complexity. Isolating the magnets from bearing heating and conductive heat loads in general becomes more difficult without using tension strap supports. The reciprocating drive is a second conductive heat source to the magnets. It must be structurally rigid to transmit large forces in tension and compression, yet not conduct heat to the magnets. Thermally anchoring some intermediate point on the drive to the shields successfully intercepts most of the heat leak.

The magnets must be capable of withstanding inertia loads greater than their weight during operation. This imposes additional constraints on internal components. The helium level must not be allowed to diminish. To do so would result in agitation of the cryogen and an increased boil-off. Therefore, the helium supply in the magnet must be constantly replenished during operation via a flexible transfer line. Even though helium replenishment can be carried out "on the fly" so that liquefier operation is not interrupted, magnet charging does necessitate a shutdown. The magnets must be motionless for charging.

\subsection{AMR Staging and Modeling}

The magnets required depend on the number of liquefier stages. The stages depend on the availability of magnetic materials capable of satisfactorily spanning the temperature range from $77 \mathrm{~K}$ to $20 \mathrm{~K}$. At present, we know of no one material capable of spanning this entire range. At least two materials, leading to a two-stage device, and perhaps three, are required. In our earliest planning, a three-stage liquefier was evaluated using a unique bed material for each stage. Materials development efforts later identified two materials capable together of spanning the full $77 \mathrm{~K}$ to $20 \mathrm{~K}$ range. The upper stage material spanning $77 \mathrm{~K}$ to $40 \mathrm{~K}$ is $\mathrm{GdNi}_{2}$. The lower stage material spanning 40 to $20 \mathrm{~K}$ is either $\mathrm{GdPd}$ or $\mathrm{Er}_{0.8} \mathrm{La}_{0.2}$. Liquefier design complexity and cost are significantly reduced with two-stage configurations. Nitrogen boil-off is slightly higher with two stages rather than three. While thermodynamic analysis suggests that a three-stage device has higher efficiency, the overall impact on efficiency using two stages is marginal. The thermodynamic gains of the three-stage configuration are largely offset by the additional losses in the added drive, pressure drops, and heat leaks.

The bed volumes required by the two-stage configuration are roughly $22 \%$ less than that for the three-stage configuration. The magnet winding volumes for either 


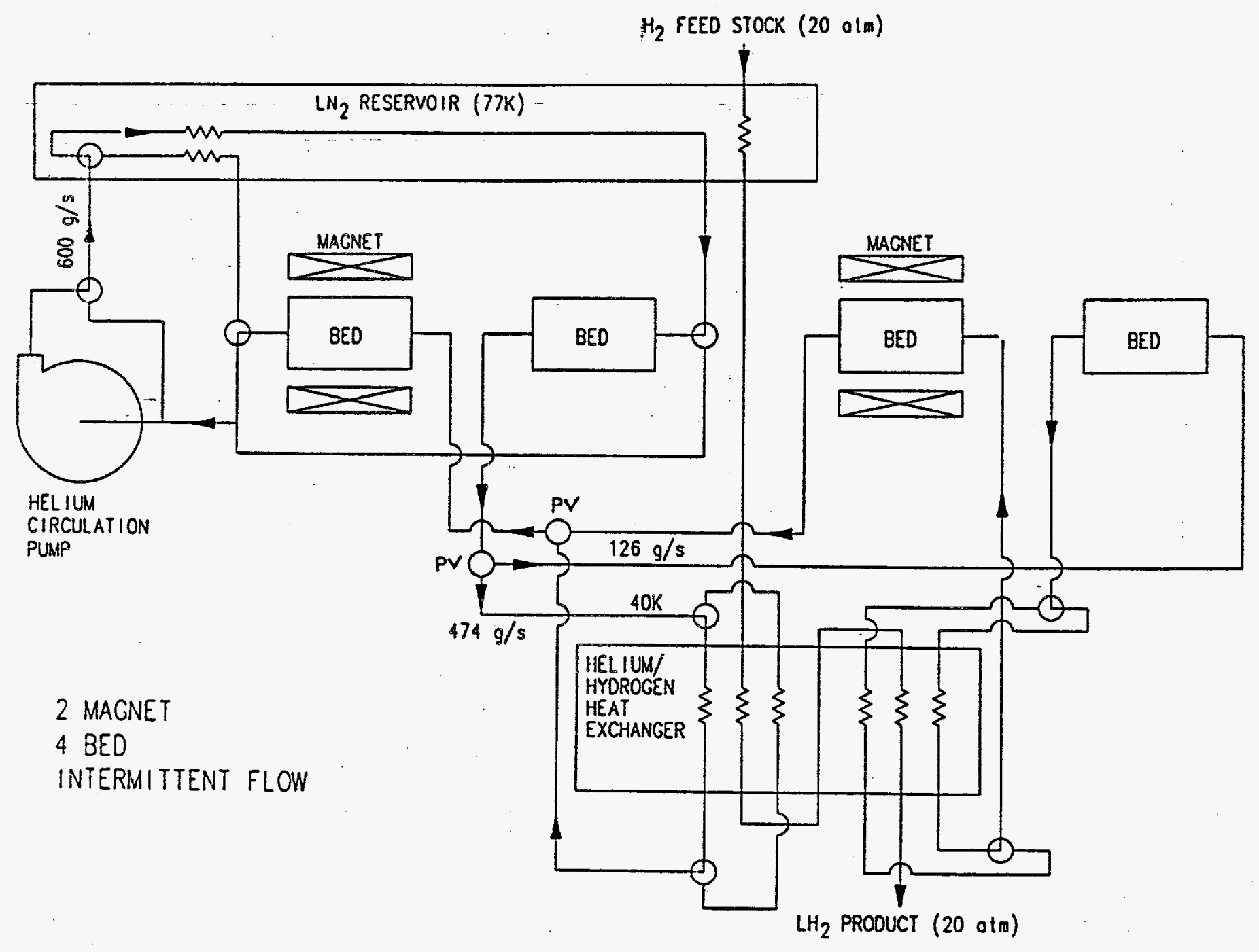

Figure 50. Preliminary Piping Schematic of a Moving Magnet Reciprocating Configuration with Flow Rectification 
rotary or reciprocating designs are reduced with the two-stage configuration. This configuration is also more compact, requiring fewer beds, valves, and piping than with three stages.

Our studies have shown that a single magnetic material will typically make a good material for an AMR in the temperature range from about the Curie point down to about half to one-third the Curie point. This applies to materials with Curie points below approximately $100 \mathrm{~K}$. Because the liquefier must span approximately a factor of 4 in temperature, a single AMR using a single magnetic material will not perform the task well, if it can perform it at all. A layered bed using multiple materials at different locations is a possible solution. A system comprised of two or more AMRs, each using a single material, is also a possibility. This system of AMRs is the approach we have chosen for the liquefier. Figure 51 illustrates the hydrogen system model, with temperatures and fluid mass flow rates indicated. Table 4 lists the operating parameters and performance. This is a single operating point. No optimization has been performed.

The liquefaction efficiency in Table 4 is based on the ideal work required to cool normal hydrogen from $300 \mathrm{~K}$ and $40 \mathrm{~atm}$ to parahydrogen at $20 \mathrm{~K}$ and $40 \mathrm{~atm}$. This ideal work is divided by the real work required by our liquefier. This includes the work required at the liquid nitrogen plant which is computed assuming an efficiency of 0.45 . 
He flow occurs half the time.

\section{$300 \mathrm{~K}$}

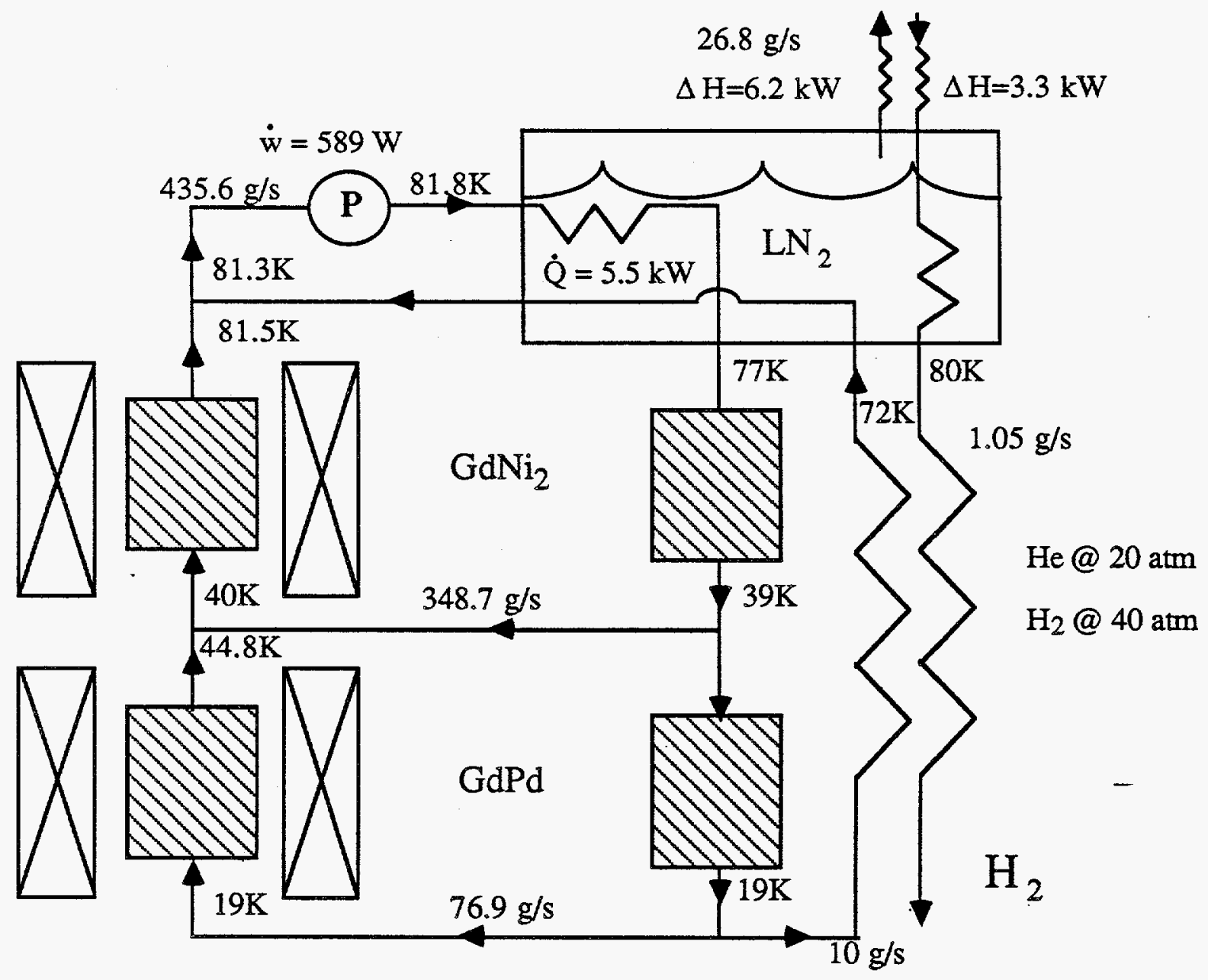

1st stage:

$$
\mathrm{V}=6.0 \text { liters } ; \dot{\mathrm{w}}=3.46 \mathrm{~kW}
$$

2nd stage:

$$
\begin{aligned}
& \quad V=2.2 \text { liters; } \dot{w}=0.64 \mathrm{~kW} \\
& \eta_{\mathrm{H}}=0.21 \text { (assuming } \eta=0.45 \text { for } \mathrm{LN}_{2} \text { plant) } \\
& 2 \\
& \eta_{\mathrm{He}}(77 \mathrm{~K}-->19 \mathrm{~K})=0.34
\end{aligned}
$$

Figure 51. Schematic of Two-Stage 0.1 Ton/Day Hydrogen Liquefier 
Table 4. Two-Stage, 0.1 Ton/Day Liquefier Model

First Stage/Second Stage

Bed length $(\mathrm{cm})$

$5 / 5$

Bed volume(l)

$6.0 / 2.2$

Bed porosity

$0.44 / 0.4$

AMR period(s)

$2.0 / 2.0$

Pressure drop(atm)

$0.19(\max ) / 0.045(\max )$

He pressure (atm)

$20 / 20$

$\mathrm{H}_{2}$ pressure(atm)

$40 / 40$

Màgnetic material

$\mathrm{GdNi}_{2} / \mathrm{GdPd}$

Particle size $(\mathrm{mm})$

$0.15 / 0.15$

Magnetic field(T)

$7 / 5$

Input work $(\mathrm{kW}) *$

$3.46 / 0.64$

Pump work (W)**

5.89

Heat rej. at $77 \mathrm{~K}(\mathrm{~kW})$

5.5

$\mathrm{N}_{2}$ boil-off(T/d)

2.6

Operating temp.

$77-40 \mathrm{~K} / 39.0-19 \mathrm{~K}$

Gas flow rate $(\mathrm{Kg} / \mathrm{s})$

$0.426 / 0.077$

Liquefaction eff.

0.21

Helium cooler eff.

0.30

* This is the work required to drive the magnet.

** This is only the work required to

overcome the pressure drop in the beds. 
The ideal work required to cool a fluid is given by the equation:

$$
W_{\text {ideal }}=\left(T_{h} \Delta S-\Delta H\right)
$$

For parahydrogen at 40 atm and $20 \mathrm{~K}, \mathrm{H}=-219.9 \mathrm{~J} / \mathrm{g}, \mathrm{S}=7.140 \mathrm{~J} / \mathrm{gK}$. For normal hydrogen at $40 \mathrm{~atm}$ and $300 \mathrm{~K}, \mathrm{H}=4243.9 \mathrm{~J} / \mathrm{g}, \mathrm{S}=55.377 \mathrm{~J} / \mathrm{gK}$. Consequently, $\mathrm{W}_{\text {ideal }}=300 \mathrm{~K}(55.377 \mathrm{~J} / \mathrm{gK}-7.140 \mathrm{~J} / \mathrm{gK})-4463.8 \mathrm{~J} / \mathrm{g}=10,007.3 \mathrm{~J} / \mathrm{g}$. For liquefaction of nitrogen at $1 \mathrm{~atm}$ from $300 \mathrm{~K}$ to $77 \mathrm{~K}, \mathrm{~W}_{\text {ideal }}=779.9 \mathrm{~J} / \mathrm{g}$. The power input at the liquid nitrogen plant is, therefore, $(26.8 \mathrm{~g} / \mathrm{s})(779.9 \mathrm{~J} / \mathrm{g}) / 0.45=46.4 \mathrm{~kW}$. The total real input work is this plus the input work to each stage and the input work for the pump: $\mathrm{W}_{\text {real }}=46.4 \mathrm{~kJ}+3.46 \mathrm{~kJ}+0.64 \mathrm{~kJ}+0.59 \mathrm{~kJ}=51.1 \mathrm{~kJ}$. The ideal work for a 0.1 ton/day plant is $(10.0 \mathrm{~kJ} / \mathrm{g})(1.05 \mathrm{~g} / \mathrm{s})=10.5 \mathrm{~kJ}$. Therefore, the efficiency is 10.5 $\mathrm{kJ} / 51.1 \mathrm{~kJ}=0.21$.

The liquefier system shown in Figure 51 is obtained in the following manner:- the specifications for the subsystem comprised of the hydrogen-helium heat exchangers with O/P converters are determined from Dr. Glen Kinard of Air Products and Chemicals, Inc. In particular, the $19 \mathrm{~K}$ outlet temperature from the second stage and $10 \mathrm{~g} / \mathrm{s}$ (half the time) helium bypass flow rate are specified. The hot temperature of the second stage is chosen, in this case, $39 \mathrm{~K}$. The AMR model is run and the flow imbalance is adjusted until the residual cooling power is zero; that is, until the average inlet temperature at the bottom of the stage equals $19 \mathrm{~K}$. The size of this stage is now adjusted to obtain the required bypass helium flow of $10 \mathrm{~g} / \mathrm{s}$. The average temperature of the helium leaving the second stage is determined.

Finding a first stage that fits the outlet and inlet conditions to the second stage requires a two-dimensional search, unfortunately. The hot temperature is $77 \mathrm{~K}$. The cold temperature and the flow imbalance have to be adjusted so that the average helium temperature emerging from the cold end is $39 \mathrm{~K}$, matching the hot temperature of the second stage, and the flow imbalance of the first stage is also $10 \mathrm{~g} / \mathrm{s}$. 
The analysis presented neglects losses due to pressure drop through pipes, valves, flow meters, and so on, as well as the circulator pump inefficiency. The analysis also neglects losses due to heat leaks through structure and radiation heat leaks. These numbers have not yet been calculated.

Equation 19 (see Section 3.0) gives the ideal adiabatic temperature change vs. temperature behavior of an AMR magnetic material. When there is a flow imbalance, the ideal behavior is no longer universal. Given the flow imbalances determined from the liquefier system point design shown in Figure 51, Figures 52 and 53 show the ideal material properties for the first and second stages compared to the real material properties. The effect of the flow imbalance is to cause the ideal material to have an adiabatic temperature change vs. temperature which is more steep than linear with a zero intercept. Because the flow imbalance is greater for the second stage, the deviation from the ideal material for balanced flow is greatest. The deviation between the real and ideal material is greatest for the second stage. Inefficiency in the second stage increases the load required by the first stage. Improvements in the material properties for the second stage should have the greatest impact on overall liquefier size and performance.

\subsection{Liquefier Scale-up Considerations}

The feasibility of subscale liquefier preliminary designs must be evaluated for both 0.1 ton/day and 1.0 ton/day liquefaction rates. The experience gained during the process of designing, fabricating, and testing the subscale liquefier should apply, as much as possible, to the full-scale device. The modular reciprocating configurations tend to scale by coupling multiple assemblies in each stage. The size of each common module would be optimized to minimize cost and complexity. The rotary configurations tend to scale simply by increasing size. Doubling the diameter of a rotary design can increase the volume available for beds by factors of four or more. Rotary configurations accommodate increasing size requirements without modular duplication.

This increasing size requirement increases the magnet winding volumes and costs. The winding volumes for the subscale rotary liquefier are significantly larger than those for the reciprocating designs. This relationship does not necessarily apply to the full-scale liquefier. 


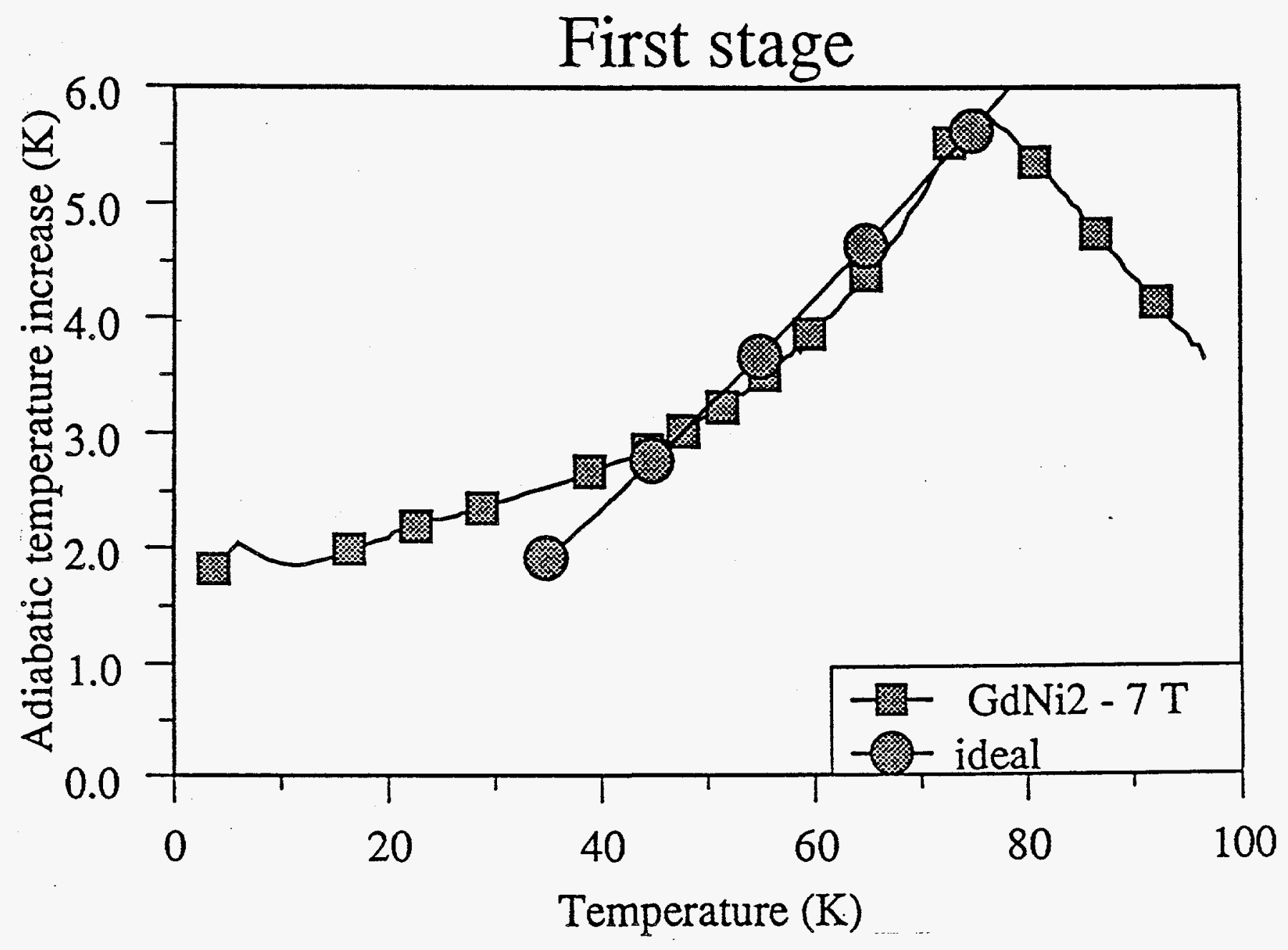

Figure 52. $\mathrm{GdNi}_{2} 7 \mathrm{~T}$ Adiabatic Temperature Change Data Compared to Ideal 


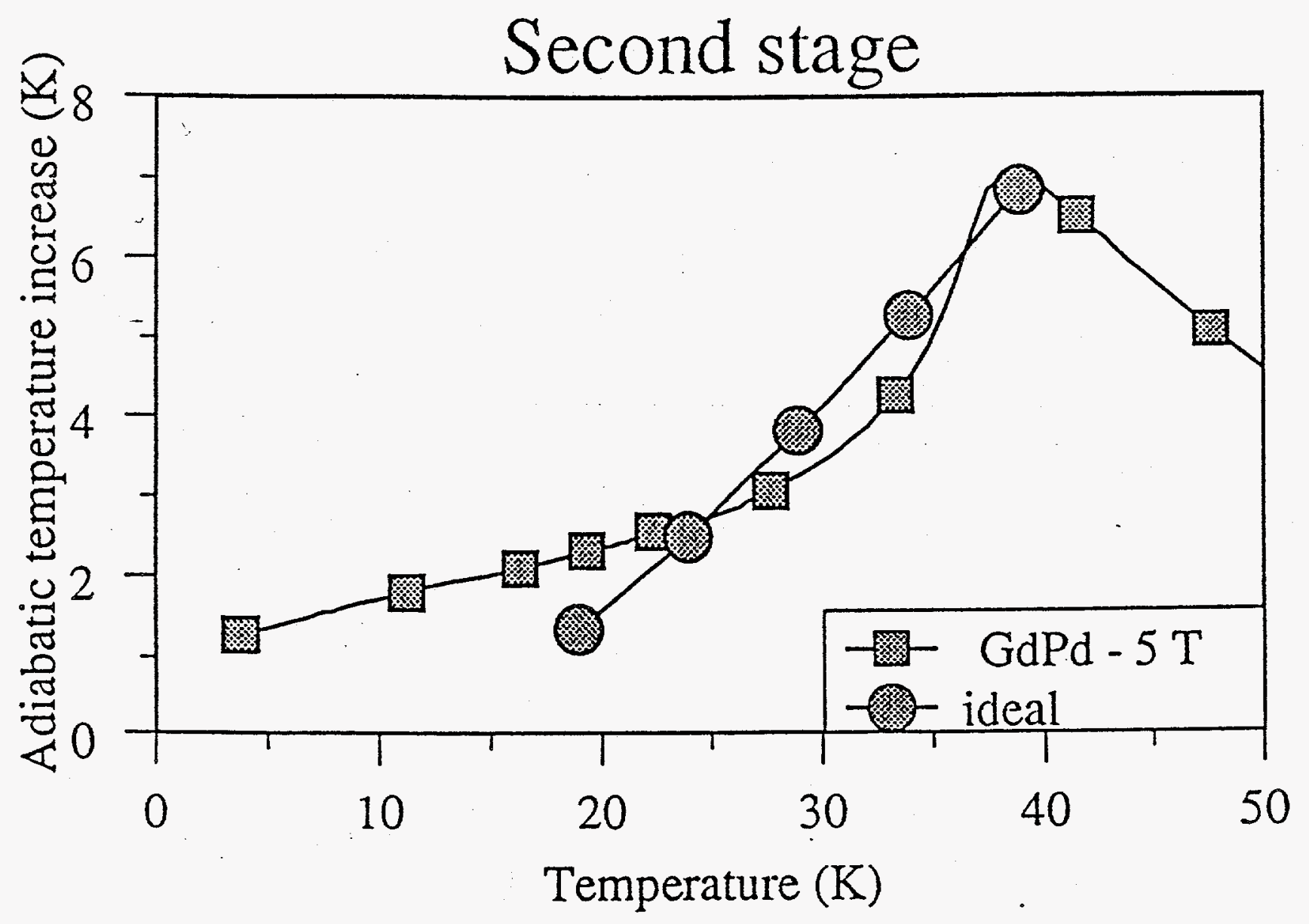

Figure 53. GdPd $5 \mathrm{~T}$ Adiabatic Temperature Change Data Compared to Ideal 
The increased cooling power of the full-scale rotary liquefier can be obtained by increasing bed volumes and rotation frequency. For example, increasing the bed volume by a factor of five and doubling the rotation frequency generates the same cooling power as increasing the bed volumes by a factor of 10 alone. Practical considerations limit the degree to which the cycle frequency of the reciprocating liquefier can be increased. The planned $0.5 \mathrm{~Hz}$ operating frequency is expected to be the highest practical operating frequency for a linear reciprocating AMR, but rotating devices are expected to operate well at higher frequencies. The implication is that the magnet winding volumes for a full-scale rotary liquefier will not increase by the same proportions as those for the reciprocating design. This makes the added flexibility of variable frequency operation offered by the rotary configuration an important scale-up consideration.

In general, continuous processes are well-suited to large scale applications. Not only does the rotary liquefier offer continuous cooling, but also near constant mechanical drive input, displaying little torque ripple. This characteristic is independent of scale. The drive fluctuations associated with the magnetic forces on large discrete masses in reciprocating designs are somewhat scale-dependent, but can be minimized by good design. Optimum bed separation and magnetic material between beds aids in minimizing drive fluctuation forces and are particularly important in scale-up.

The same circumstances that generate fluctuating drive forces in reciprocating designs also introduces $\mathrm{AC}$ losses in the magnets. Varying magnetic mass within the magnet induces current fluctuations in the windings that manifest themselves as losses. Rotary designs maintain a near constant mass of magnetic material within the high field region of the dipole magnets so $\mathrm{AC}$ losses are insignificant and independent of scale. Reciprocating AC losses are somewhat scale-dependent, but again can be minimized by good design as described previously.

The most important scale-up considerations can be summarized as follows:

- magnetic material volume,

- magnet winding volume,

- operational envelope,

- compactness,

- drive losses \& fluctuation,

- $\mathrm{AC}$ and eddy current losses, and

- helium flow rates

\subsection{Preliminary Design Selection Criteria}

The objective of the liquefier preliminary design phase was twofold. First, several potential AMR configurations were conceived and evaluated. A set of 
evaluation criteria was used to conduct a comparative analysis of the most promising configurations. These included a two-stage reciprocating magnet AMR and a twostage, single housing, rotary AMR. Finally, one configuration was selected to be carried forward through the detailed prototype design phase.

The criteria are configuration-dependent. In other words, criteria that addressed components or systems common to both configurations, and hence did not differentiate among them, were not considered. For example, both configurations require a vacuum system. This system would not vary in any significant way from one configuration to another. Therefore, vacuum systems were not comparatively evaluated.

The preliminary design evaluation criteria considered are:

- magnet complexity and cost,

- magnet cooling and charging,

- $\quad$ ability to be scalable,

- piping system complexity,

- regenerator bed material volume and cost,

- risk or the degree of proven technology applied,

- thermal performance, and

- fabrication complexity and number of parts

While several configurations were easily rejected, of those remaining no obvious winner emerged. This is testimony that every design requires compromises. For magnet complexity and cost, the reciprocating design is preferred for both sub and fullscale liquefiers. It uses solenoid magnets, which are inherently simple and can be fabricated with confidence. These magnets are less expensive for the subscale liquefier, but may lose this edge in the full-scale device.

The rotary configuration is preferred for magnet cooling and charging. Permanent charging and helium line connections can be made to the stationary magnets in the rotary configuration. The reciprocating configuration requires flexible helium transfer lines and demountable leads. Although both features have been successfully implemented in the past, they add cost, complexity, and additional failure modes.

The magnets for the rotary design consisted of solenoidal coils with a "racetrack" shaped cross section. Heat transfer occurred only in the high field region. A significant portion of the rotary wheel must then be in either the high field or the low field region. In past designs the high field region only covered about $1 / 6$ th of the wheel circumference rather than the desired 1/3. This issue was addressed and resolved by increasing the wheel diameter from $70 \mathrm{~cm}$ to $90 \mathrm{~cm}$ and by incorporating two thermodynamic cycles per wheel revolution rather than one. The enlarged diameter provided clearance between the magnets and the wheel/housing hub. Adding a second cycle per revolution doubled the number of magnets but also significantly 
enhanced the overall field profile. Figure 54 depicts a possible magnet configuration for the rotary design.

A typical field profile is depicted in Figure 55. There are two curves plotted, labeled geometry B and geometry H. Geometry B is the profile for the standard fourmagnet coil set for the two-cycle per revolution configuration. Geometry $\mathrm{H}$ is the field profile with two additional field shaping coils toward the center of the wheel. This is the most promising profile obtained and requires a third pair of trim coils.

Several wheel configurations have been investigated. In each case a set of magnets was configured and the magnetic fields determined in both the radial and circumferential directions. Some economies of overall magnet volume can be made by reducing the number of stages. However this tends to increase the size of the individual magnets.

The rotary configuration is a clear winner in ability to be scaled. It offers a far broader operating frequency range, uses less Regenerator bed material, and operates continuously. The magnets for the reciprocating design may be less expensive, but this is not clear.

The rotary configuration is also preferred for piping system complexity. No bidirectional flows occur that would require flow rectification or directional control valves although a pipe flow modification identified in the preliminary design review eliminates the flow rectification issue. The rotary piping system is less complex and uses fewer components than the reciprocating arrangement. These are conventional, time-proven components that add little risk. Therefore, this criteria carries little weight.

The reciprocating configuration is preferred for bed construction and cost. The bed volumes for the subscale liquefiers are independent of design. However, the beds for the reciprocating design are simple fabrications composed of a collection of parallel wafers. The beds in the rotary design are embedded in a complex wheel fabrication not well-suited to interchangeability. Installing new beds in the reciprocating design is simple. This is an important feature for development hardware. 


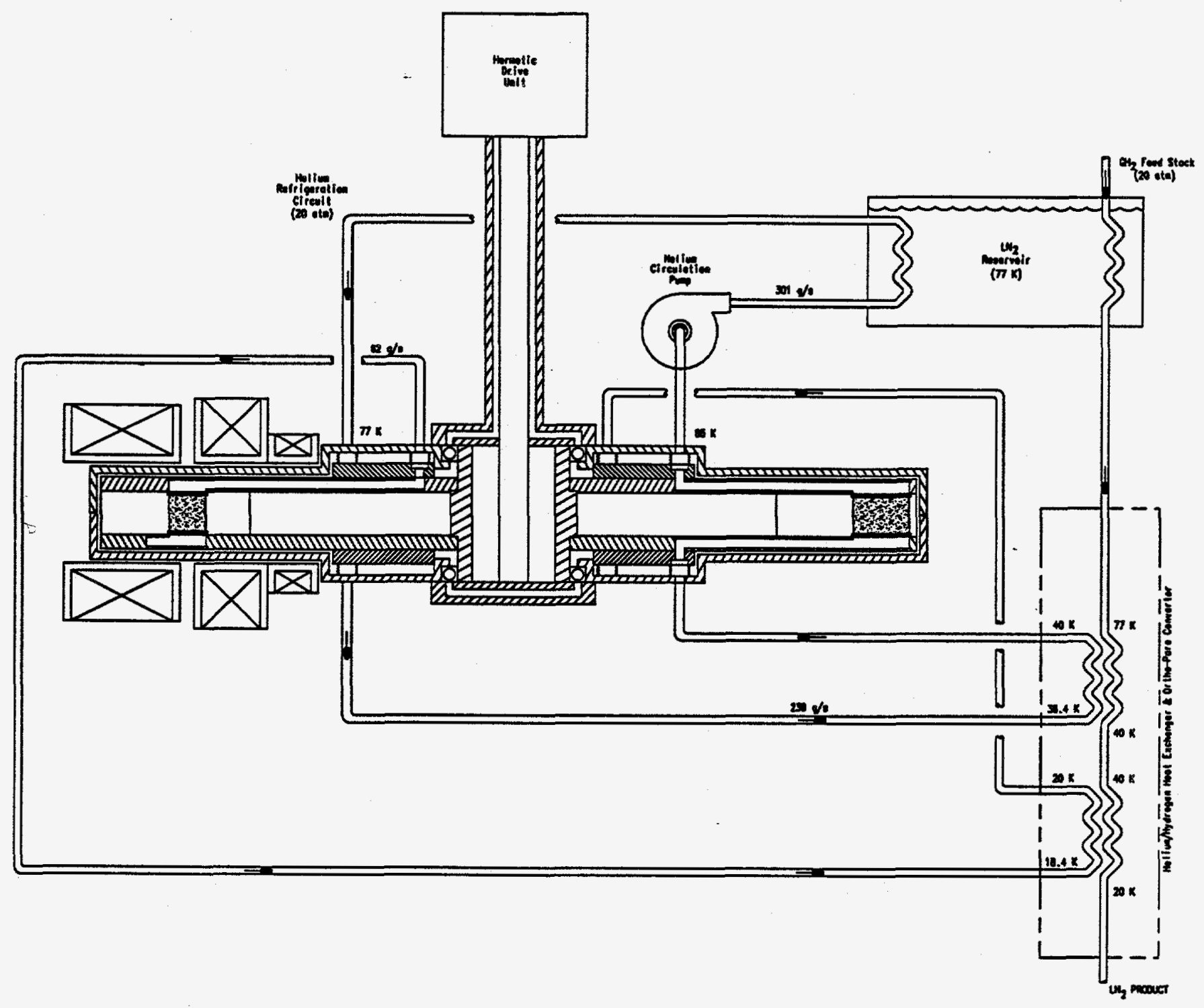

Figure 54. Magnet Configuration for the Rotary Design 


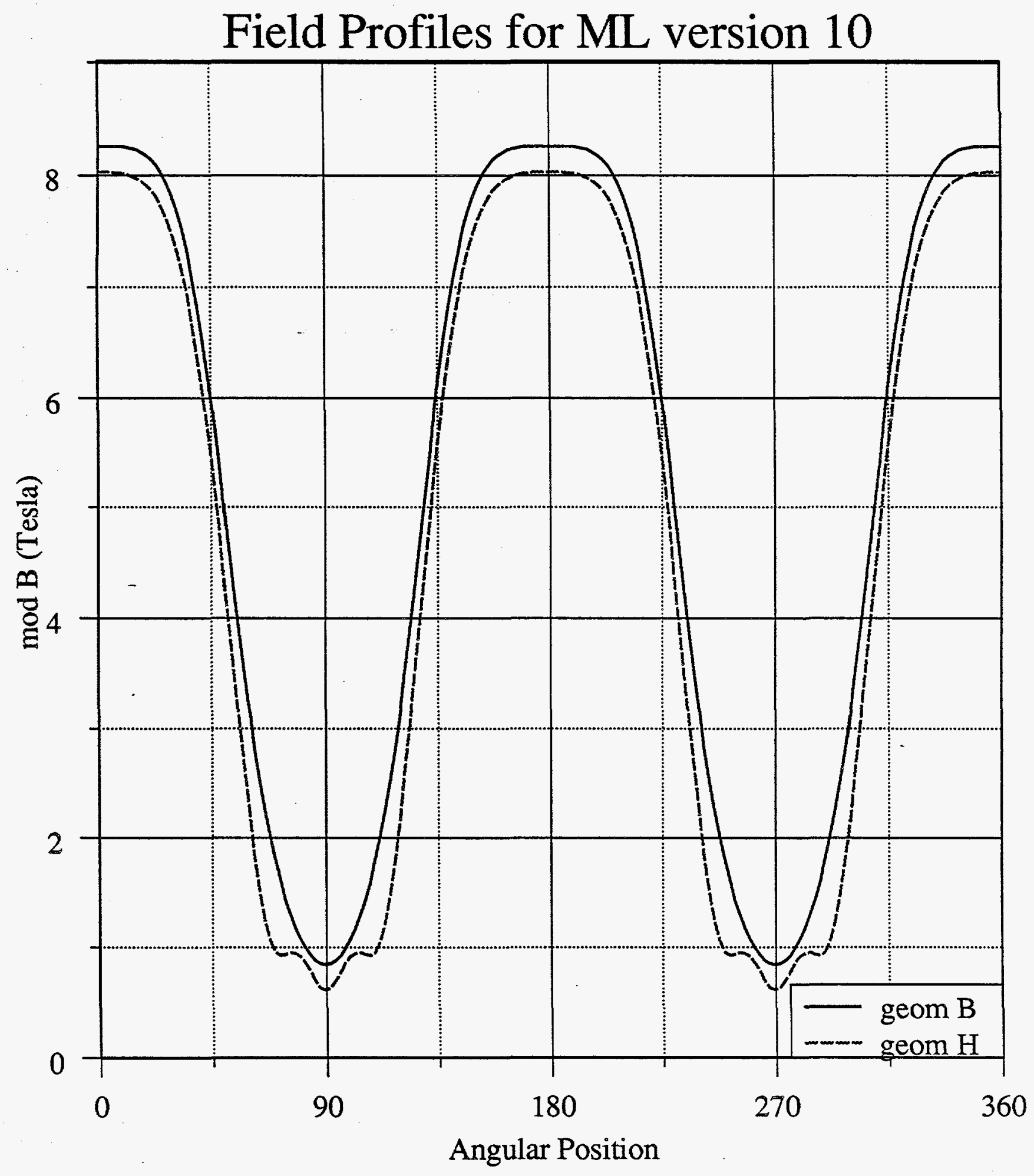

Figure 55. Typical Field Profile 
Work on magnets for the rotary design was discontinued in January of 1991 when it was determined that the concept was, with present technologies, more risky than the linear reciprocating design.

The reciprocating configuration is clearly superior for risk and the degree of proven technology applied. It is based on a working demonstration prototype and contains no high risk design elements. Performance sensitivity is not tightly coupled to any one component in the design as it is to seal integrity and reliability in the rotary design. This criteria is most important. First and foremost, the liquefier must work. Further, higher risk technology development to enhance future designs should proceed in parallel with, but not in place of, low risk demonstration hardware.

The cooling power of the two configurations will meet specifications and direct comparison of this criteria is meaningless. However, other aspects of performance do discriminate between the two. Continuous cooling is preferred over pulsed or intermittent cooling. Continuous drive work is preferred over variable drive input. Overall liquefier efficiency may be slightly higher if a continuous process is used. Based on these performance criteria, the rotary configuration is preferred.

The reciprocating configuration is preferred for fabrication complexity and number of parts. All major components are composed of low-precision subassemblies. The bed structure is a simple cylindrical construction well-suited to internal pressurization. The magnets are simple solenoids mounted to frames fabricated from standard shapes. The rotary configuration requires a complex wheel fabrication supporting many beds and complex flow manifolding; this assembly requires a large number of joints and is not well-suited to bed swapping. Furthermore, the rotary housing structure is an inefficient pressure vessel subject to deflection while simultaneously maintaining precision bearing alignments. The rotary design is a more complex fabrication requiring more parts and higher precision than the reciprocating design.

The preliminary design evaluation based on the selection criteria identified the reciprocating configuration as the best subscale liquefier design. The rotary configuration uses more expensive racetrack magnets rather than simple solenoids. It sustains very high structural loadings because of internal pressure. The wheel and housing fabrications are complex with many structural joints, susceptible to leakage. Most importantly, its performance is very sensitive to leakage flow past the dynamic rotary seals. Seal life and durability in this application is unproven, and thus risky. The rotary configuration is well-suited to scale-up and should not be completely discarded. A development effort to demonstrate a reliable dynamic seal should proceed so that a rotary design may be considered for the full-scale liquefier.

The reciprocating design is based on proven hardware, thereby minimizing risk. This first magnetic liquefier for hydrogen must successfully liquefy hydrogen if any second generation, large-scale designs are to be developed. These collective analytical 
and design developments were presented to a team of external reviewers at a preliminary design review with the recommendations that the two-stage reciprocating design was our first choice for further development. The following table summarizes the preferred configuration for each of the eight selection criteria.

Table 5. Preferred Configuration Based on Selection Criteria

Subscale Design

Selection Criteria

Magnet Complexity

and Cost

Magnet Cooling and

Charging

Ability to be Scalable

Piping System

Complexity

Regenerator Bed Material

Volume and Cost

Risk or Degree of Proven

Technology Applied

Thermal Performance

Fabrication Complexity

and Number of Parts

$\mathbf{X}=$ Preferred Choice

Rotary

$\mathrm{X}$

N/A

X

X
Reciprocating

X

Full-scale Design

Rotary

Reciprocating

$\mathrm{X}$

X

$\mathrm{X}$

X

X

X 


\subsection{Preliminary Design Review Modifications}

Only one significant modification to the proposed two-stage reciprocating liquefier design resulted from reviewer comments after the preliminary review. With this modification (described below), a change in the distribution of cold helium flow from the AMR results in an improved hydrogen/helium heat exchange, regardless of AMR configuration. This simplifying change improves cycle efficiency and eliminates the need for helium flow rectification.

The modified piping configuration is illustrated in Figure 56. As depicted in the figure, helium flow passes through the demagnetized first stage bed and splits. Approximately $20 \%$ of the flow passes on to the second stage demagnetized bed while the remainder removes heat from the magnetized first stage bed and returns to the pump. Flow leaving the second stage demagnetized bed is again split between the hydrogen-helium heat exchanger with ortho-to-para catalytic beds (O/P Heat Exchanger) and the magnetized second stage bed. Approximately $6 \%$ of this flow is diverted to the O/P Heat Exchanger. These flows also return to the pump inlet. 


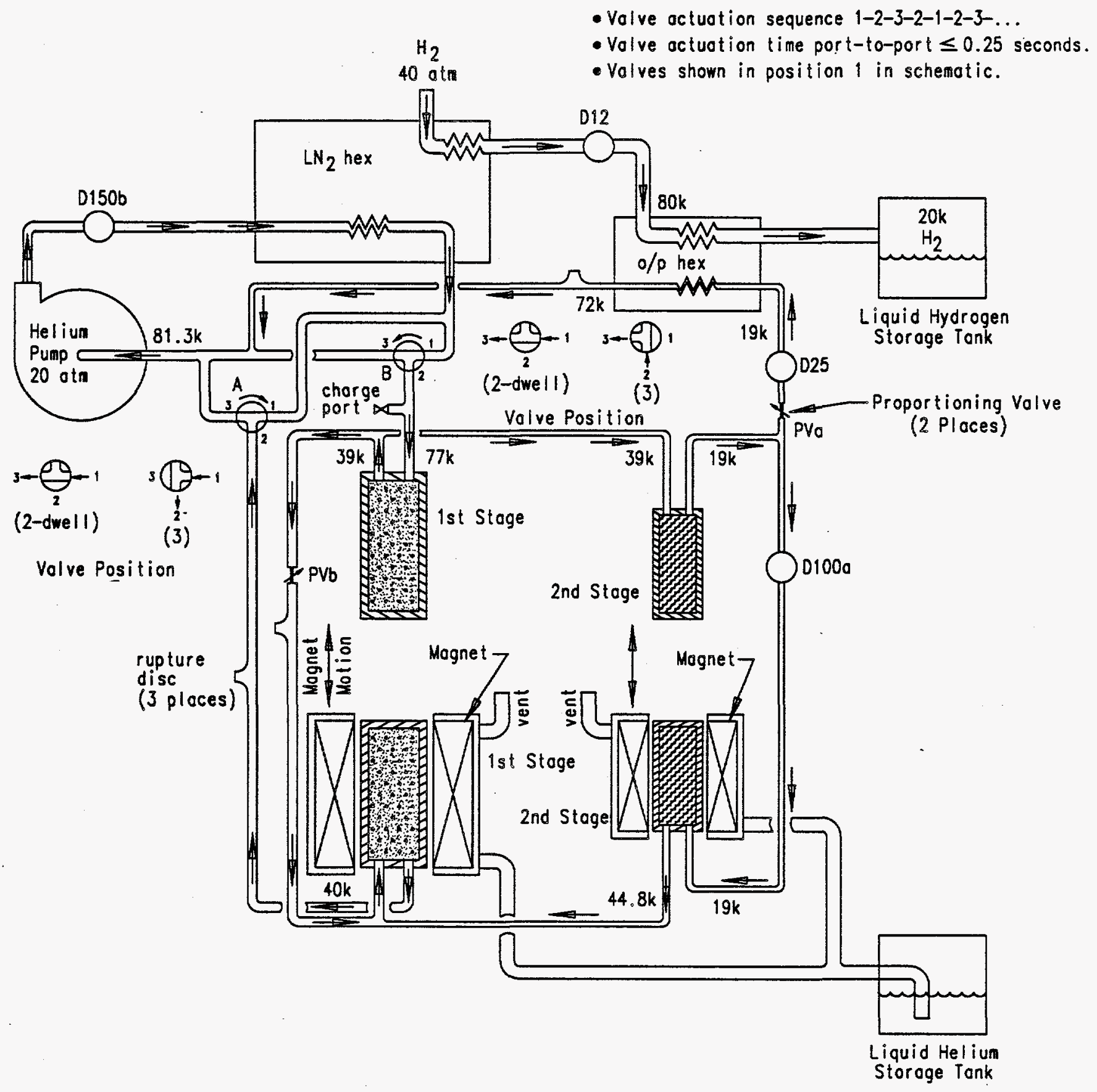

Figure 56. Final Prototype Piping Schematic 
Helium enters the O/P Heat Exchanger at roughly $19 \mathrm{~K}$ and counterflows with hydrogen feedstock which enters at about $80 \mathrm{~K}$. Helium flow through the O/P Heat Exchanger does not change direction when flow through the rest of the liquefier is reversed. This flow arrangement results in unbalanced mass flows through the beds. A larger mass flow passes through the demagnetized beds than through the magnetized beds because some flow is diverted to the O/P Heat Exchanger without passing through the magnetized beds. This flow imbalance would result in warming of passive regenerative beds. The varying heat capacity of active magnetic beds, however, mitigates this reaction to flow imbalance. Modeling results indicate efficiency increases of about $40 \%$ over the previous flow arrangement.

\section{Detailed Design of a 0.1 Ton/Day Magnetic Liquefier}

\subsection{Configuration Component Integration}

The modified piping configuration is shown incorporated into the liquefier structure with magnets and drive in Figure 57. The components in the system have been arranged to allow for removal of the beds, magnets, and drive with minimal disturbance to other hardware. The removal of an individual bed assembly involves shifting one structural cross beam, unbolting of the secondary drive tubes (but not removal), and disconnection of the four flanged pipe joints to the beds. The magnet and bed are simply lifted together sufficiently high to allow the bed assembly to slide out of the magnet bore and clear the structure. The upper radiation shield must be removed and the vacuum vessel rolled out of the way.

The drive can be easily removed by unpinning the main drive tube from the yoke and disconnecting the feed-through housing from the vacuum vessel. The vacuum connection to the housing, hydraulic lines, and instrumentation connections must be removed before the cylinder/feed-through subassembly is free. The Yoke and secondary drive tubes can be removed without first removing the main drive tube and feedthrough. All other components are welded in place and do not require removal unless a failure occurs. These components include the valves, flow meters, heat exchangers, and circulation pump. They are conventional, highly reliable components not requiring frequent replacement or service.

The drive loads to the magnets are reacted, through the magnetic field, by the beds. This reaction force is transferred from the beds to the support structure and back through the feed-through to the hydraulic cylinder. The beds are supported only at each end to minimize any thermal heat leak into them from the structure. The heat leaks are estimated to be $200 \mathrm{~mW}$ and $100 \mathrm{~mW}$ for the first and second stages, respectively. The details of the interface between bed assemblies and support structure are illustrated in Figure 58. This support interface provides radial bed adjustment to 

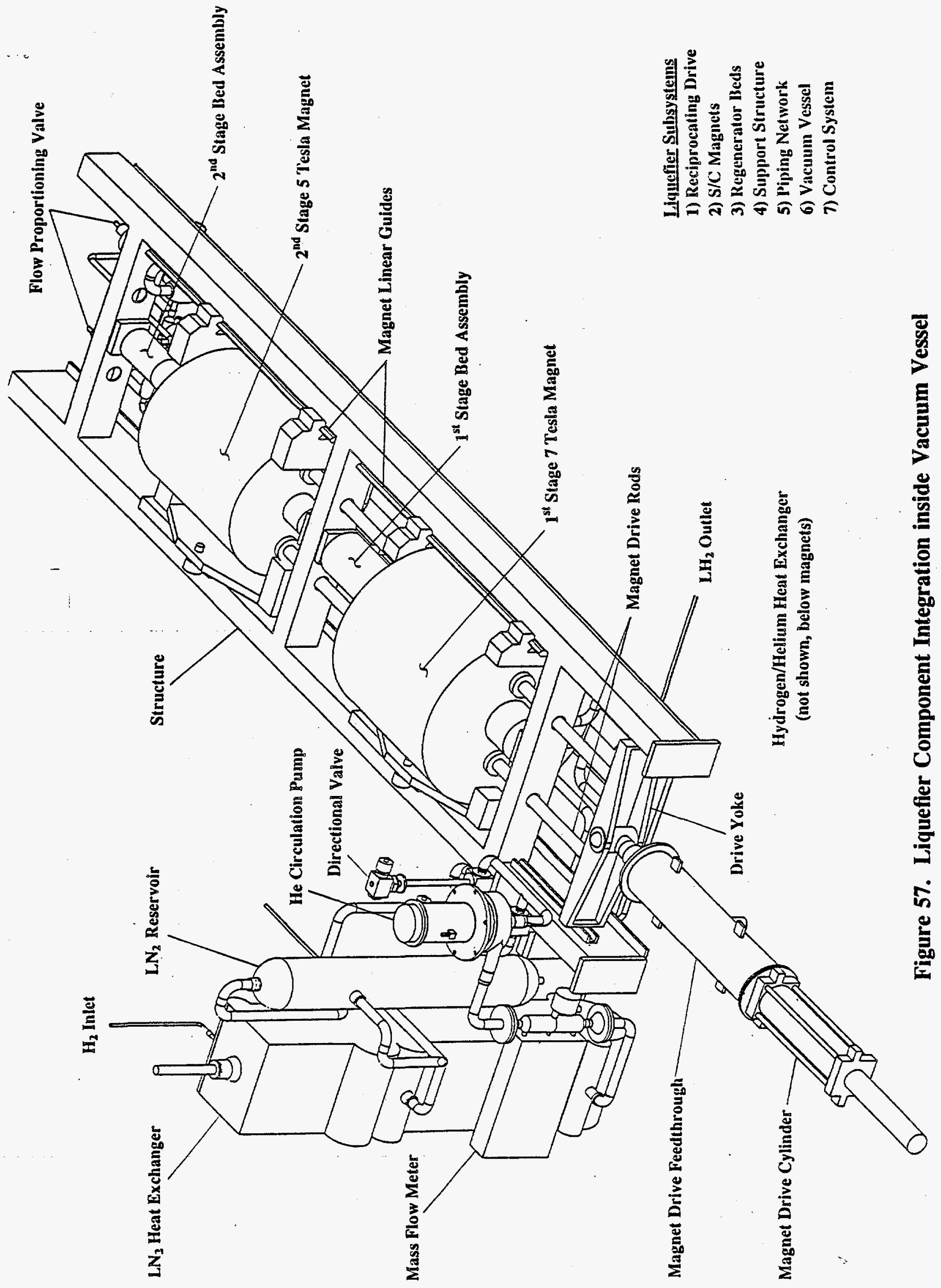
center the bed assembly in the magnet bore.

In addition to isolating the bed assemblies from the support-structure cross-beams, thereby limiting contact conductance, these supports also provide the load path between the beds and structure. A square stud extends from the end of the beds to mate with a square pocket. A spherical tip on the stud engages a belville spring to compensate for any off-axis misalignment of the bed. The square pocket, once secured, prevents possible rotation of the beds on their axis. The spring compensates for the differential contraction between the G-10 bed assemblies and stainless steel support structure. The first and second stage beds shrink 0.02 inches and 0.01 inches less than the structure from room temperature. At assembly, the removable cross beams are adjusted so the springs have only 0.02 and 0.01 inches remaining before bottoming out. During cool down, this clearance is absorbed by the differential contraction occurring between beds and structure so that a solid interface for drive load transfer is created. If the springs do not bottom out, the beds will synchronously shift slightly with the magnets. This would potentially fatigue joints and perhaps lead to leaks. To avoid this, the 0.02 and 0.01 dimensions are actually slightly less than the true differential contraction expected between components. The differential contraction induced compressive loading in the bed assemblies without this feature would be approximately 10,000 pounds. The bed assembly mounting calculations are summarized in Appendix A.

The predominant differential contractions throughout the remainder of the system occur between the brazed aluminum heat exchangers and piping, and between the bed assemblies and piping by induced differential contraction strains in the piping, particularly at the joints, can cause leaks. All leaks are significant because of the high pressure helium in the system. The joints are therefore welded wherever possible to maximize structural integrity of the piping system. Also, flexible bellows couplings are used to compensate for the differential contractions and eliminate the potential for high strains.

The thermal load on the beds and piping system is minimized by effective isolation techniques, including thermal radiation shielding. Because all major components are integrated to the support structure, it is important that it be well-controlled thermally. To achieve this, the two main structural longitudinal members are flooded with liquid nitrogen. This stabilizes the temperature of these members so they become a stable platform on which to mount hardware. The semicircular radiation shields are anchored along the length of the longitudinal members creating a $77 \mathrm{~K}$ "tunnel" encapsulating the magnets, beds, and piping.

Mechanical and thermal contact between the support structure at $77 \mathrm{~K}$ and the vacuum vessel wall is limited to point contacts at bearings and pinned joints. These interfaces carry large loads with marginal contact conductance, minimizing the thermal 

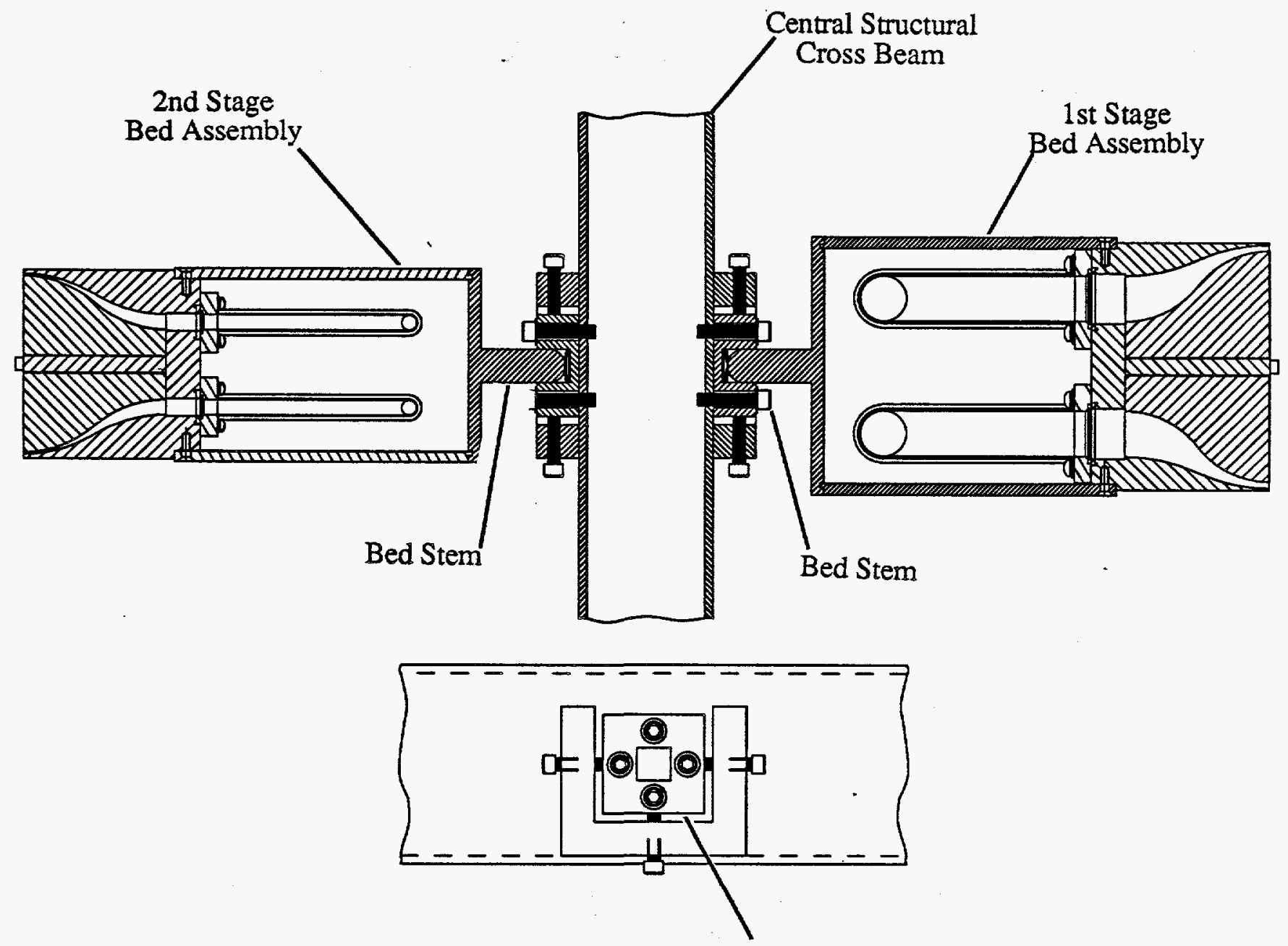

Adjustable Bed Stem Pocket

Figure 58. Bed Assembly Interface to Support Structure 
leak to the structure and conserving $\mathrm{LN}_{2}$. The drive end of the structure is mounted to the vacuum vessel wall by two pinned joints located at the longitudinal members. All drive loads are transferred through these two joints. A series of small rollers support the structure along its length, carrying much of the weight. These rollers are mounted to the interior of the cylindrical vacuum vessel that surrounds the magnets, beds, and structure. This can, in turn, rolls on a track in the foundation. The structure is secured to the front half of the vacuum vessel by the pinned joints and remains stationary. As the vacuum can rolls back away from the fixed front half of the vessel, the small rollers on its interior traverse the length of the longitudinal members. Once the vacuum can is withdrawn to its extreme open position, the structure is supported by one roller. The support structure and all components mounted to it are then completely exposed, on all sides, for maximum accessibility. Just as with the pin joints, the rollers introduce small heat leaks to the $77 \mathrm{~K}$ structure.

Most components mounted to the structure operate at temperatures between $77 \mathrm{~K}$ and $20 \mathrm{~K}$ so the heat leaked from the structure to them is small, particularly with good isolation. The superconducting magnets, however, must operate at $4 \mathrm{~K}$. Thermal isolation between the magnets and structure is especially important to minimize helium boil-off. The magnets couple to the structure through a three-point linear guide arrangement. Guide rails attach to the structure with insulating mounts. Linear bearings and followers ride on these rails to guide the magnets. A single follower on one side mounts to a long G-10 beam simply supported at each end of the magnet. This beam provides generous thermal isolation between the magnets and structure. The very small contact conductances through the bearing also serve to isolate the magnet. The estimated heat leak through this support is $100 \mathrm{~mW}$. The pair of linear bearings on the side opposite the follower offer less isolation because they do not benefit from the long G-10 beam and house four small roller bearings each. Therefore, there are more contact conductances to transmit heat to the magnet. The linear bearings are estimated to conduct $200 \mathrm{~mW}$ each to the magnets from the $77 \mathrm{~K}$ support structure.

The remaining components including the circulation pump, $\mathrm{LN}_{2}$ precooler, and directional valves are all housed within the front, stationary half of the vacuum vessel. Heat leaks and structural loading on these components are not significant. Overall, considerable attention has been placed on the structural and thermal loading in the liquefier. Structural loads impact reliability and thermal loads impact thermodynamic performance. Attention to both is required to ensure success.

\subsection{Working Magnetic Bed Design}

Each of the two stages is comprised of a pair of bed assemblies. The assemblies are composed of a stack of four individual bed modules housed within three concentric thin wall G-10 tubes. The bed modules are the fundamental component of the bed assemblies. They sustain large magnetic-induced loads, pressure loads, and thermal gradients. 
An exploded view of one bed module of the first stage bed assembly is illustrated in Figure 59. The bed module tube is capped on both ends with felt, screen, a perforated disk, and a flow header. The flow header channels helium between the bed volume and annular flow cavity in the bed assembly. These half-moon shaped channels are exposed only along half the header perimeter, as detailed in Figure 60. These exposed channels are the ports into the bed. The header distributes helium flow over the entire bed surface. The ports on opposite ends of the module are oriented $180^{\circ}$ from each other. This is because the two crescent-shaped annular flow cavities in the bed assembly must each couple to a unique port. Features of an assembled module including port orientations are detailed in Figure 61.

The flow dividers inside the bed module tube serve two functions. As the name implies, they help to divide and channel the helium flow through the bed. They also serve a structural role in carrying compressive magnetic loads from adjacent beds. All four modules stacked in the bed assembly experience varying attractive forces toward the magnet. The forces on the bed farthest in the stack from the magnet are superimposed onto the beds between it and the magnet. Each bed in turn superimposes additional loads onto the bed nearest the magnet. These forces accumulate to roughly 48,000 pounds of compressive loading on the bottom bed in the stack. Much of this load is carried by the flow dividers rather than as a compressive load on the sphericalpacked bed materials, preserving the mechanical integrity of the particles. The bed assembly stack is illustrated in Figure 62.

The figure shows the annular flow cavities between the bed stack and outer tube. Two helium ports located at the top of the assembly provide for flow into and out of each of the four beds. Flow from a port passes down through the $180^{\circ}$ annular flow cavity and enters the side ports in each module. The flow passes through each bed and exits the assembly via the second $180^{\circ}$ annular flow cavity. Pressure drops are kept to a minimum by incorporating generous port geometry with large cross-sectional flow areas to keep velocities down where practical. These pressure drops are estimated to be $37.9 \mathrm{kPa}(5.5 \mathrm{psi})$ and $10.3 \mathrm{kPa}(1.5 \mathrm{psi})$ for the first and second stage, respectively. 


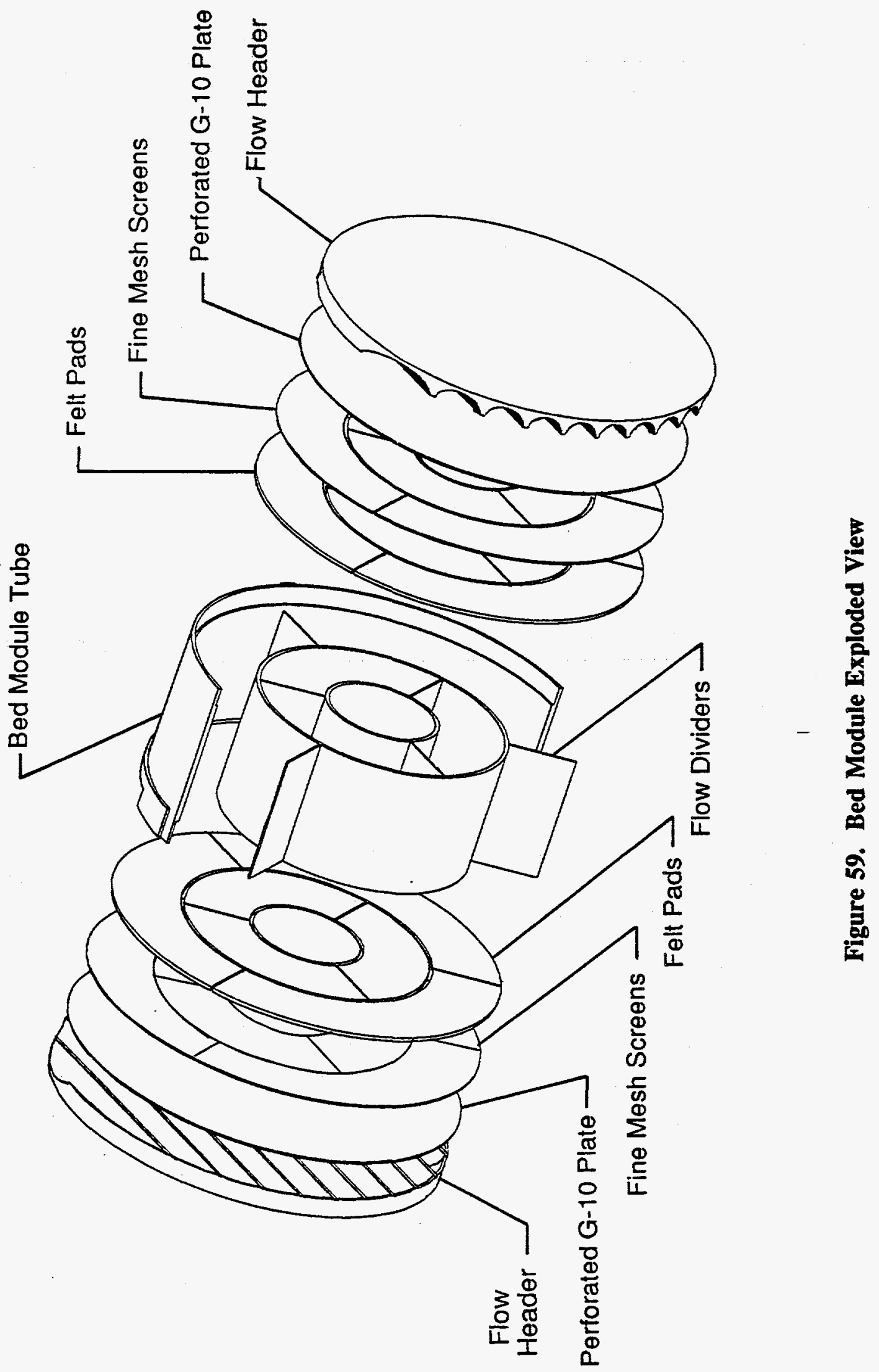




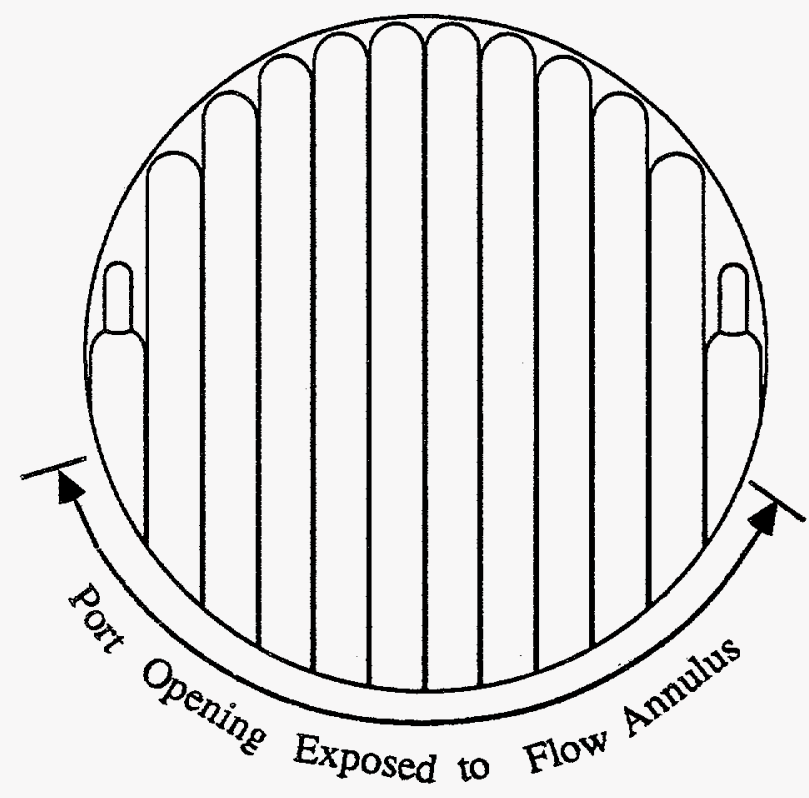

৫শর্রেশ

Figure 60. Bed Module Flow Header 

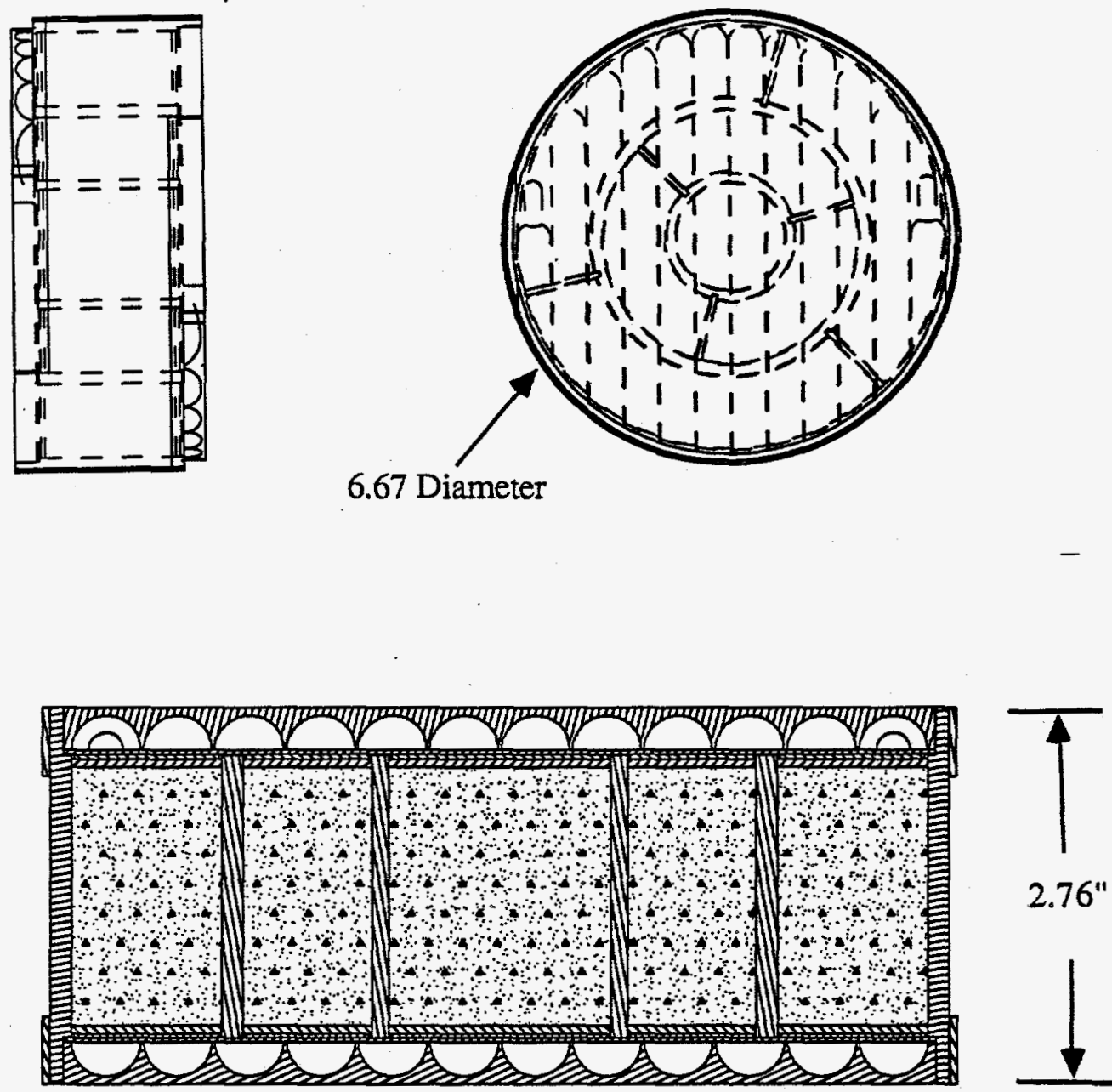

$2.76^{\prime \prime}$

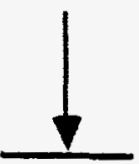

Figure 61. Bed Module Port Orientation and Features 


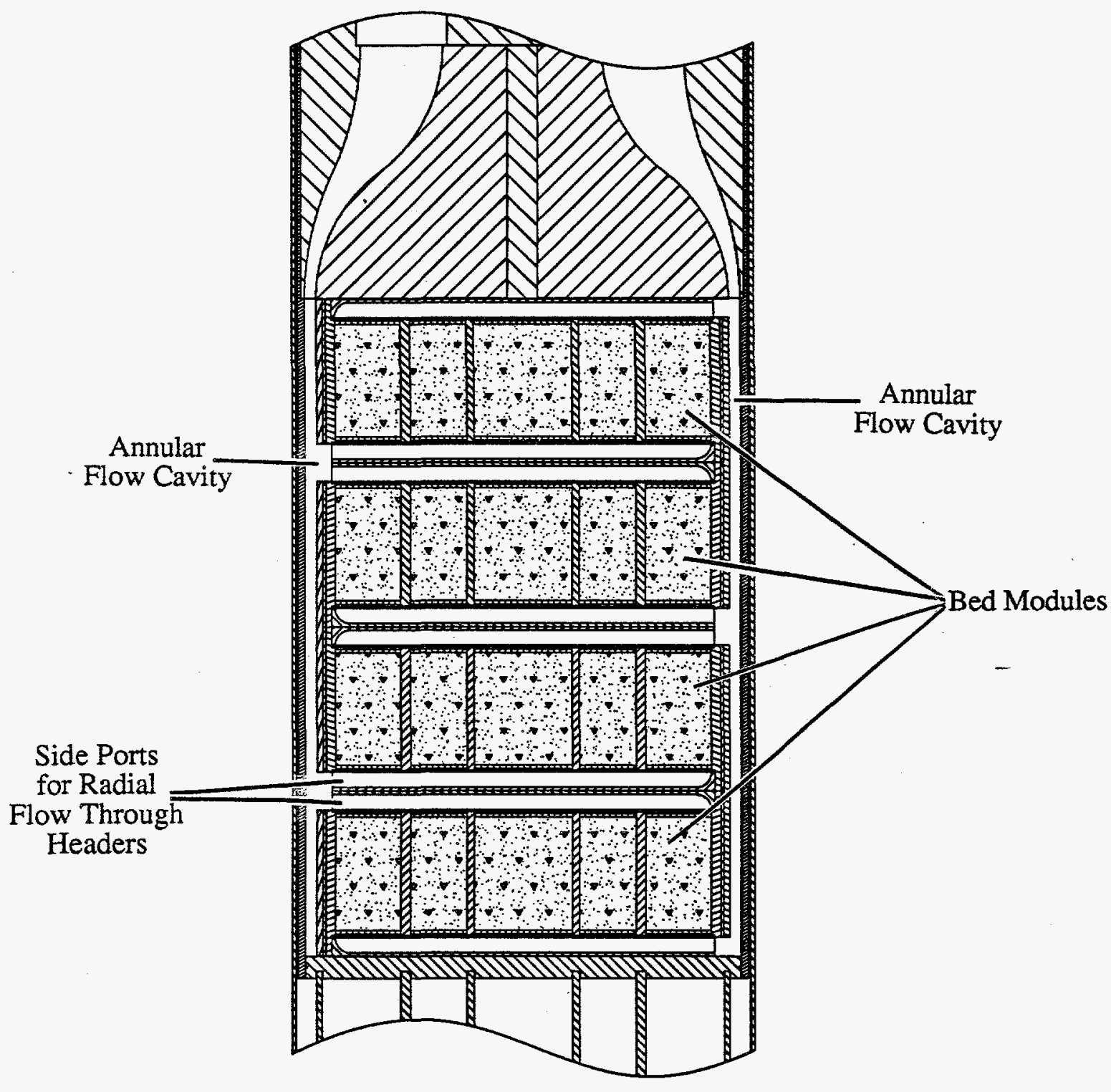

Figure 62. Stacked Bed Assembly and Flow Paths 
The work required to compensate for pressure drops is not the only loss mechanism in the bed assembly. Internal parasitic heat leaks, primarily by conduction, must be controlled. The warm and cold helium gas occupying the annular flow cavities see an axial temperature gradient along the bed module tube walls. The warm gas will transfer heat to the cold regions of the wall, reducing the net cooling power of the bed. The cold gas leaving the assembly has heat transferred to it by the warm regions of the bed module tube walls, again reducing the net cooling capacity of the exiting gas. This heat leak is significantly reduced by using a double-wall module design. Once the four bed modules are stacked together, two semicircular sleeves are bonded to them. This structurally couples the modules while creating the double wall. The 0.16 $\mathrm{cm}(0.06 \mathrm{in})$ radial gap thus created is occupied by trapped helium from the cold side of the beds. Helium gas is a poor thermal conductor and this essentially stagnant gas acts as an insulator between the two walls. This space is not exposed to the vacuum environment and therefore does not entail any leakage risk. The estimated heat leak in the first stage beds without the double wall is $307 \mathrm{~W}$. Adding the double wall reduces this leak to an acceptable value of $60 \mathrm{~W}$. These calculations are documented in Appendix B. The heat leaks in the second stage bed are smaller because the temperature differentials and exposed surface areas are roughly 50\% smaller than in the first stage beds.

Two double-walled bed assemblies sealed into a G-10 support tube create one complete single stage assembly. The first stage bed assembly is illustrated in Figure 63. It is supported by the posts at each end. All structural loads reacted by the drive system must pass through these end supports. Regardless of where the magnet is positioned the loading in this assembly is compressive. This significantly enhances the reliability of the bond joint seals. Only six bond joint seals between vacuum and the $2068 \mathrm{kPa}$ (300 psi) helium are used in each stage. Four of these six are for the piping connections. The pipe joint design decouples structural and sealing requirements, significantly reducing risk. Features of this joint are detailed in Figure 64. A small stainless steel ring is bonded to the G-10 port cap. A C-ring seal element is compressed between this ring and the pipe flange by the bolts. Any strain on the joint during handling or from vibration is carried by the bolt joint rather than the bond. This joint is expected to perform well with high reliability. - The two remaining bonds in the assembly are between G-10 cylindrical shapes. The joints have very large bond areas both for strength and sealing integrity. 


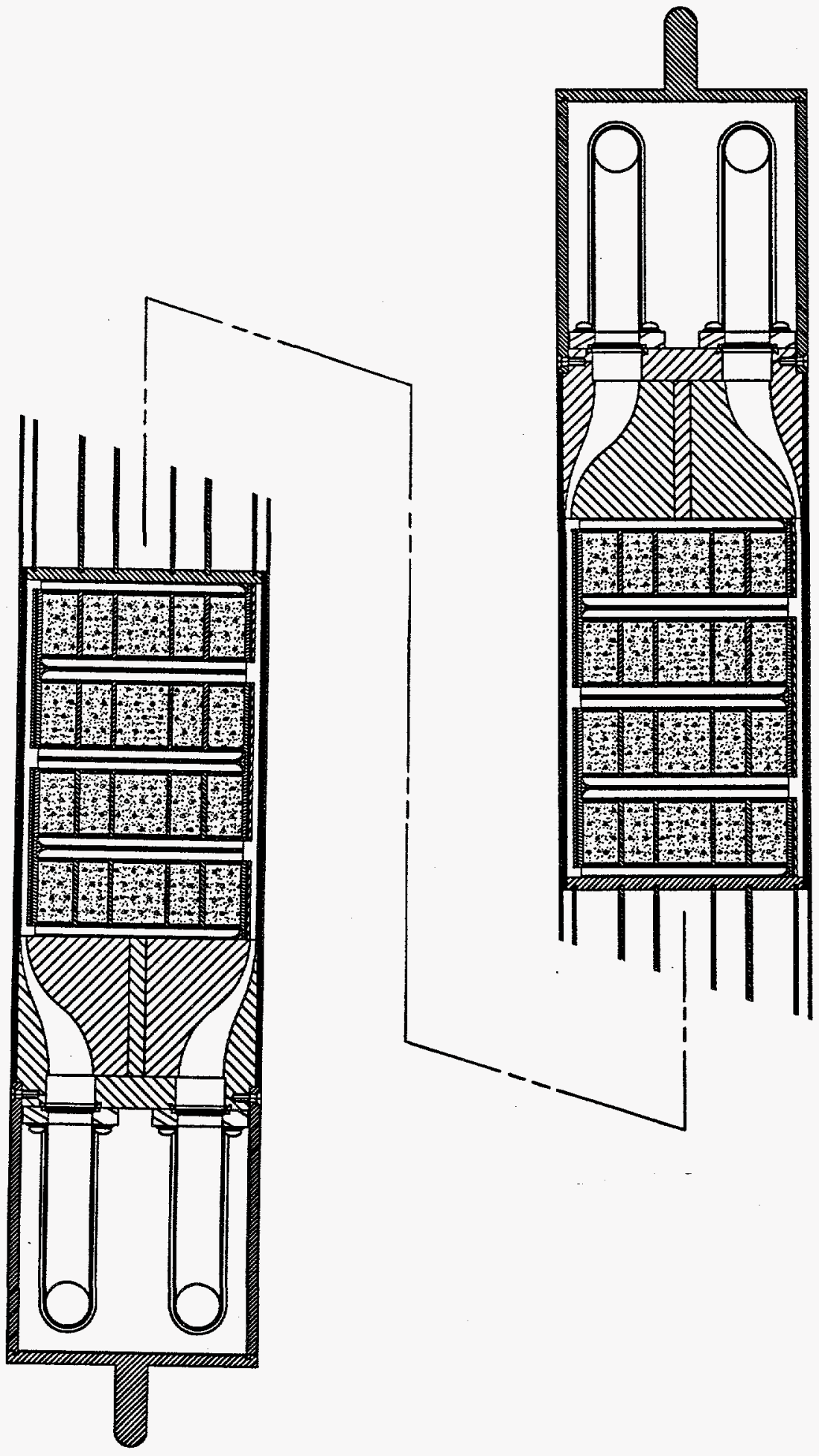

Figure 63. Bed Subassemblies Sealed into a G-10 Support Tube Structure 


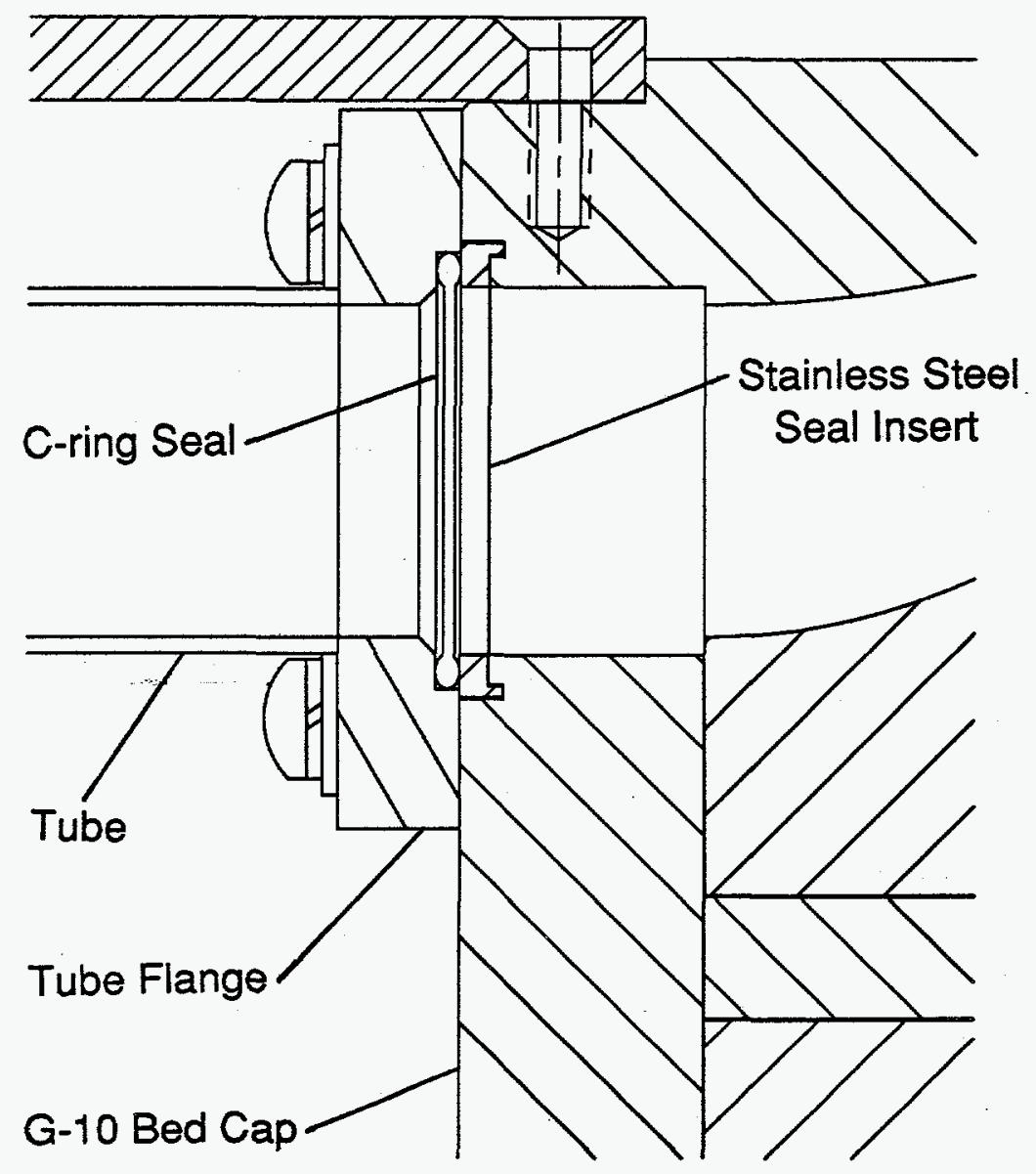

Figure 64. Bolted Tubing Joint to Bed Assembly 
Structural analysis of the bed design was carried out using SDRC I-DEAS. This software package integrates solid modeling, finite element modeling, and post processing. Initial bed modeling used simple shell and solid elements and was run on both the SDRC linear finite element solver on a SUN workstation and UAI NASTRAN on an Astronautics ZS mini-supercomputer. The results were compared and found to be essentially identical. All later structural modeling was conducted with the SDRC software.

More detailed modeling of the beds used linear quadratic shell elements for all of the structure, except the spacers that create the double wall around the bed module. The spacers were modeled with linear brick elements. The model consisted of five concentric tubes. The inner flow divider tubes and bed module tubes carried loads generated by the magnetic forces. The bed module wall was also loaded with a preload corresponding to the loads expected from bed packing. The outer tube was loaded with the $2068.5 \mathrm{kPa}(300 \mathrm{psi})$ helium pressure. The flow headers were modeled as shell elements and provided a load transfer mechanism between beds. The model was refined by substituting parabolic elements for the linear elements. The parabolic elements reflect geometry more accurately and give more accurate results, particularly if coarse meshes are used. The change to parabolic elements agreed well with the previous solution. The SDRC optimization package was used to determine the wall thicknesses for all bed tubes. All stress levels are at or below $68.95 \mathrm{MPa}$ (10000 psi). Glass epoxies such as cryogenic grade G-10CR essentially exhibit no yield strength. Rather, only tensile ultimate strengths are published. The typical values for the strength of these materials at $77 \mathrm{~K}$ are at least $60 \mathrm{KSI}$ in the fill direction and $120 \mathrm{KSI}$ in the warp direction. Compression strengths are higher than these values. The results of this modeling instill high confidence in the structural integrity of the $\mathrm{G}-10$ bed assemblies.

Figure 65 illustrates the complete analysis model of a bed module. The model includes the flow dividers, bed module tube, module tube sleeve, and outer support tube. Under the expected loading conditions as previously described, a maximum stress of less than 10000 psi occurs in the midlength region of the bed module tube wall (the absence of colors in the figure makes it difficult to differentiate the contours). This analysis was repeated for a worst case scenario in which the trapped space between the bed module tube and sleeve becomes evacuated due to a leak. In this case, a maximum stress of 21000 psi occurs again in the bed module tube wall. This stress remains well below the tensile strength of the material. This leak could not occur without other leaks occurring first, tending to cancel its effect. Therefore, this is a somewhat artificial worst case scenario.

Essentially all internal bed bond joints are loaded in compression. Failure of one of these joints will not result in structural collapse of the assembly. Failure would however cause internal leaks (none to vacuum) that could influence performance. Therefore, the stress levels in the joints are designed to be low by using large bond surface areas. 


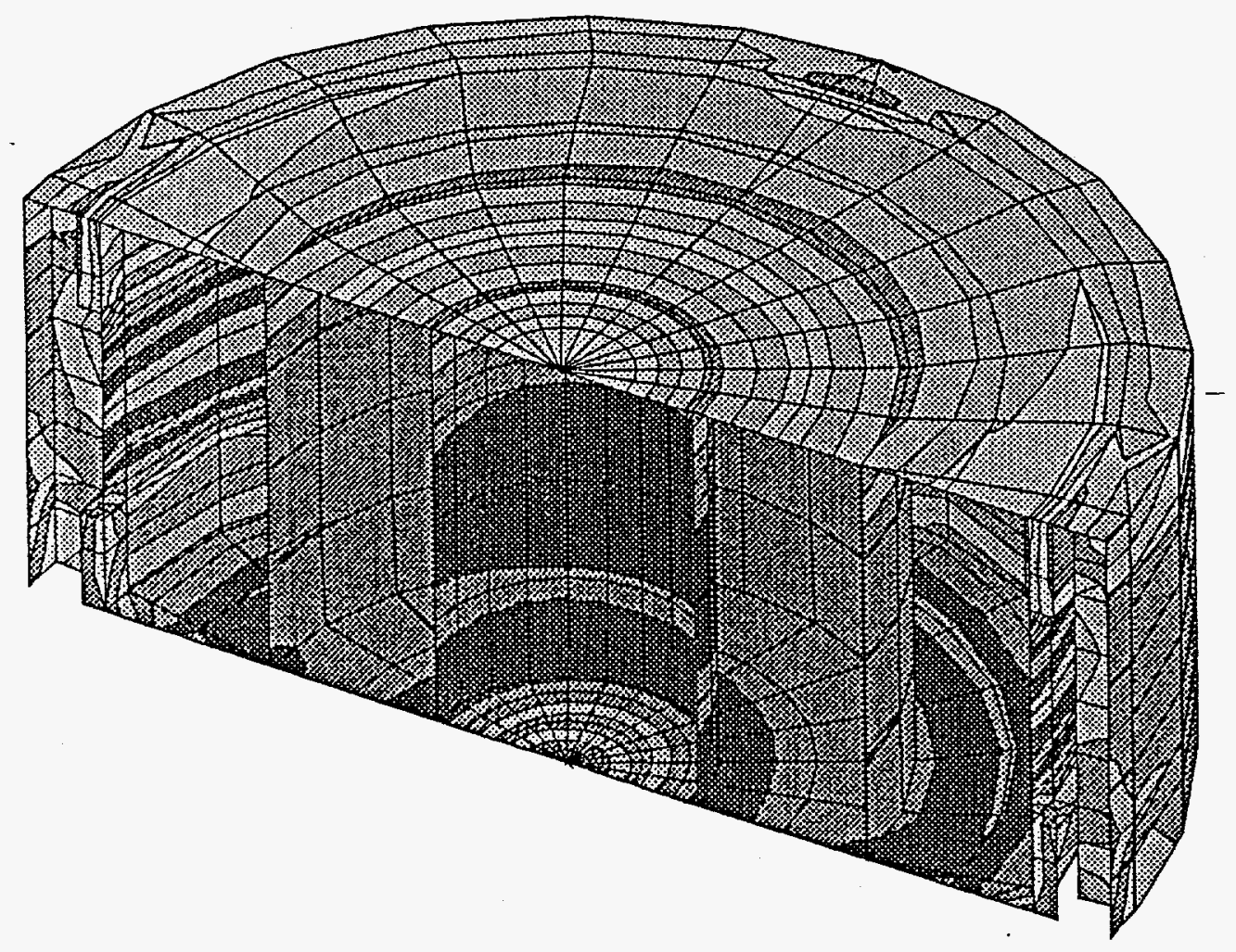

Figure 65. Bed Assembly Stress Contours 


\subsection{Reciprocating AMR Magnet Design}

The design of the one-tenth scale device centered on two concepts, a rotary and a reciprocating design. Astronautics performed initial design calculations for the magnets of each design concept. It is intended to let a bid specification for the magnets.

Two magnets are required for the reciprocating AMR. One magnet for each, the first and second stage. These magnets are simple solenoids and have a "warm" bore. That is, the high field bore of the magnet is available in which beds can be placed.

The magnets must be designed to minimize mass and cost. Based on the AMR staging and modeling analysis discussed in Section 4.6, the first stage magnet must operate at $7.3 \mathrm{~T}$ so the bed can produce the maximum cooling. The engineering balance struck in the design was between magnet stroke and size. A small bore magnet, whose outside diameter is small, can be easily fabricated to provide high fields. However, because of the need for a fixed volume of bed material the length of the bed becomes long and, therefore, causes both the magnet length and its actuation stroke to be excessive. Excessive stroke causes increases in the hydraulic drive system requirements, including ram size, pump size, and power. It also increases the acceleration seen by the magnet during each cycle.

Magnets fashioned for large diameter, short length bores, rely on a larger winding volume to generate the desired field magnitudes and profile. This also causes undesirable increases in the hydraulic drive system requirements.

Both the first and second stage magnets will be under a dynamic reciprocating load. These force variations must be considered when building the magnets. The first and second stage magnets are physically coupled together and are reciprocated by a single drive. Each magnet shuttles between the two beds in its respective stage. Attractive and repulsive magnetic forces on these beds can be very large but can be reduced to a manageable level by trading off between bed separation distance and minimum allowable low field (bed in demagnetized state). The higher the allowable low field, the closer the beds may be spaced and the lower the drive forces. However, as the low field rises, cooling power drops. The magnetic forces associated with the first stage beds are much larger than those associated with the second stage. Figure 66 is a graphical depiction of the net system drive forces, based on an 18 inch $(46 \mathrm{~cm})$ bed separation distance.

The magnets will also see a variable permeance in the bore. Magnet losses will occur in the coil winding because of the changing magnetic field associated with the varying permeability in the bore. Calculations of these losses will be performed by the magnet manufacturer as well as Astronautics personnel. The addition of permeable materials in the bore, other than the bed materials, is being considered to smooth the variability in permeance. However, the addition of these materials will affect the bed 


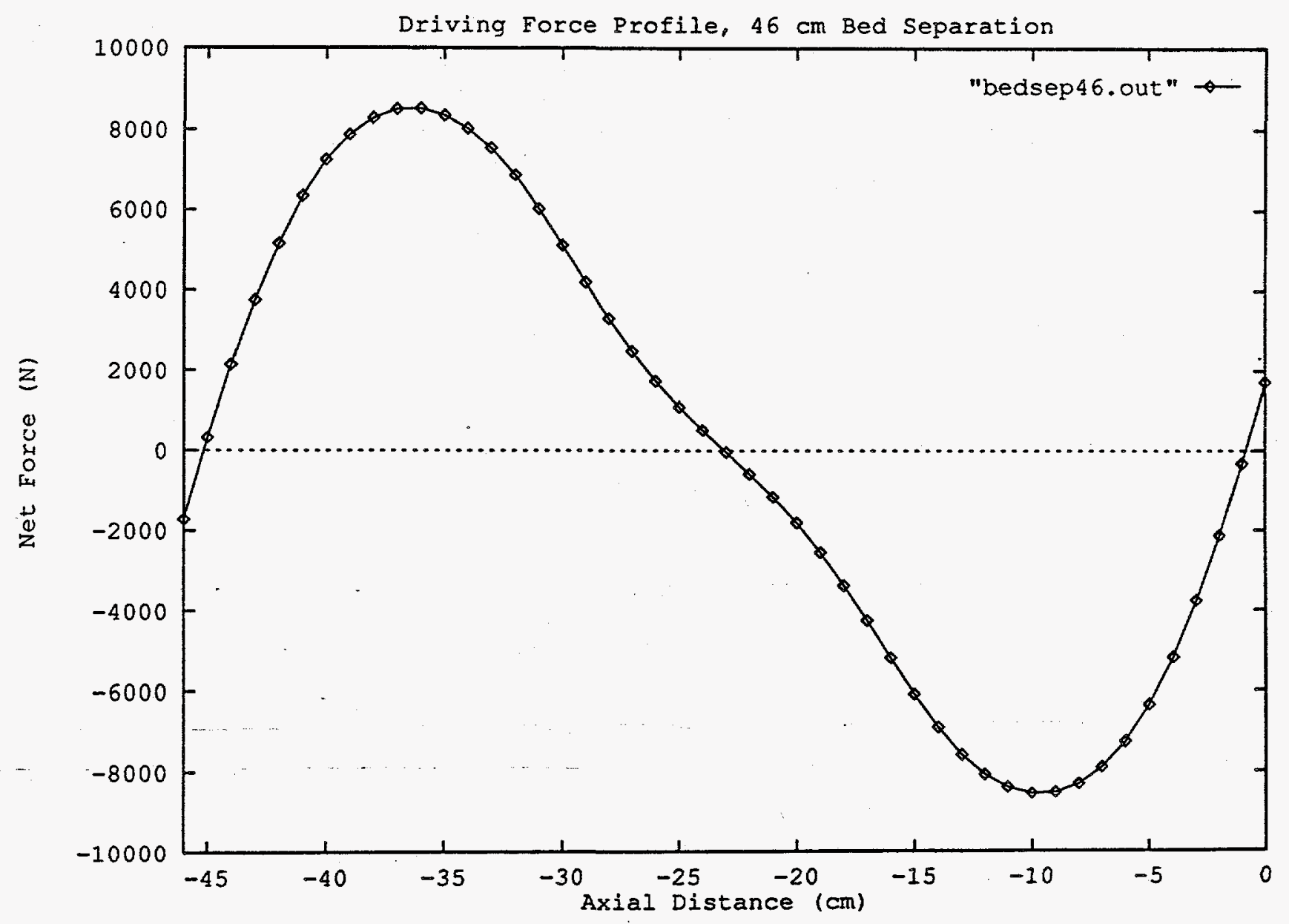

Figure 66. System Drive Forces 
separation and, therefore, hydraulic drive distance. Again, the engineering trade-offs are between coil losses (and duration between magnet recharge) and in power/mechanical drive requirements.

\subsubsection{Magnet Design Specifications}

It is intended that a major superconducting magnet supplier will design, fabricate, and test the magnets necessary for the prototype unit. During the detailed design of the reciprocating magnet liquefier design, we have been working with Oxford Instruments North America, Incorporated, a reputable company with a long history of providing magnets of high quality. The large superconducting magnets, in reciprocating motion, cause unusual dynamic loads on support structures and winding.

The present design specifications are outlined in Table 6. Oxford Instruments North America Incorporated has worked closely with us to determine a design specification that can be built with low risk and cost.

\subsection{Auxiliary Equipment}

All the major auxiliary systems external to the bed assemblies will be supplied by vendors whose expertise lies with these systems. These systems include the superconducting magnets, drive system, piping components, vacuum system, and structure. These systems/components are specified to a level of detail sufficient for the vendors to design and fabricate hardware for final assembly integration at Astronautics. A listing of major components and vendors is delineated in Table 7. The vendors listed may change as a result of competitive bidding conducted during the actual Phase II fabrication effort. 
Table 6. Hydrogen Liquefier Magnet Specifications

\begin{tabular}{|ll|}
\hline 1st Stage Magnet & \\
\hline Central Field & 7.3 Tesla \\
Operating current & $<=100$ amps \\
Homogeneity & $\pm 3 \%$ over 10 inches on axis \\
Cancellation & 0.7 Tesla at $\pm 13 \mathrm{x}$ inches on axis \\
Stray field & 0.1 Tesla at 13 inch radius off axis, around magnet \\
& perimeter along its entire length \\
He vessel clear bore & 7.7 inches dia. usable \\
Magnet \& vessel weight & 500 lbs max. \\
He vessel & non-vacuum jacketed, entire magnet \& He vessel \\
& will be housed inside a large vacuum vessel \\
& approximately 16 inch diameter X 30 inches long \\
Dynamics & magnet will reciprocate 18 inches every $2 \mathrm{~s}$ \\
& while in persistent mode \\
Magnet includes & persistent mode switch, quench protection, \\
& temperature sensors, He level sensor \\
Magnet leads & superconducting, demountable for reciprocating \\
& operation \\
Persistent mode duration & 7 days minimum \\
Heat leak & $1 \mathrm{~W}$ conductive heat leak into He vessel radiation from \\
& $77 \mathrm{~K}$ shield surrounding magnet \\
\hline 2nd Stage Magnet & \\
\hline same as above, except He vessel & \\
clear bore 5.7 in. dia. usable & \\
and 5.5 T central field & \\
(See Note 1.) & \\
\hline
\end{tabular}

\section{Notes:}

1. Second stage magnet can be identical to first stage if cost effective.

2. Ancillary equipment to include liquid helium storage dewar, with minimum 7-day helium supply, flexible helium transfer line connecting helium dewar to reciprocating magnets, and magnet power supply.

At present only one magnet manufacturing company has bid on the magnet specification. Others will be invited to do so. The supplier of the magnets will also design and fabricate the demountable leads, the persistent mode switches, the helium fill lines, the helium envelope for the magnets, and the remote helium dewar necessary for replenishment of helium in the magnet structure. 
Table 7. Major Components and Vendors

\begin{tabular}{|c|c|}
\hline composite main drive tube & AMALGA \\
\hline composite secondary drive tube & AMALGA \\
\hline Hepco linear bearings for magnet guide & EMCO \\
\hline hydraulic cylinder load cell $(50,000 \#$ cap $)$ & EATON/Lebow \\
\hline servo-controlled hydraulic system module & NEFF Eng. \\
\hline bellows seal & HEECO \\
\hline liquid nitrogen precooling heat exchanger & ALTEC \\
\hline ortho-para helium/hydrogen heat exchanger & ALTEC \\
\hline helium circulation pump w/electronic drive & Barber Nichols \\
\hline cryogenic ball-type directional valves & FLODYNE \\
\hline pressure/temperature sensor interface & Keller PSI \\
\hline 600 psi hydrogen pressure/temperature sensors & Keller PSI \\
\hline 300 psi helium pressure/temperature sensors & Keller PSI \\
\hline hydrogen flowmeter/transmitter $(1 \mathrm{~g} / \mathrm{s})$ & Rosemount \\
\hline helium flowmeter/transmitter $(5 \mathrm{~g} / \mathrm{s})$ & Rosemount \\
\hline helium flowmeter/transmitter $(87 \mathrm{~g} / \mathrm{s})$ & Rosemount \\
\hline helium flowmeter/transmitter $(436 \mathrm{~g} / \mathrm{s})$ & Rosemount \\
\hline flowmeter controller/interfaces & Rosemount \\
\hline nitrogen compressor (cryo valve pilot pres) & American Bristol \\
\hline cryogenic flow proportioning valve $(1 / 2)$ & CVI \\
\hline cryogenic flow proportioning valve (1.5) & CVI \\
\hline C-ring face seal (various sizes \& spares) & PSI/EG\&G \\
\hline 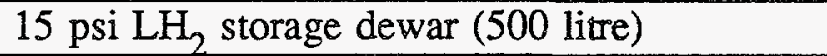 & CRYOFAB \\
\hline 7T S/C magnet w/dewar \& lead joints (7.7) & OXFORD \\
\hline 7T S/C magnet w/dewar \& lead joints (5.7) & OXFORD \\
\hline magnet power supply & OXFORD \\
\hline S/C magnet power leads (demountable) & OXFORD \\
\hline flexible helium transfer line & OXFORD \\
\hline liquid helium storage dewar (250 litre) & OXFORD \\
\hline vacuum system module & CVC Products \\
\hline vacuum vessel & Cleveland Ent. \\
\hline $77 \mathrm{~K}$ thermal shields & McFarland Mfg \\
\hline stainless steel support structure weldment & Kasperson Mfg. \\
\hline
\end{tabular}




\subsection{Drive System}

\subsubsection{Hydraulic Unit}

The drive system is subdivided into two subgroups. These include the digital servo-hydraulic subgroup and the mechanical drive linkage subgroup. The hydraulics serve as the prime mover, generating the forces necessary to translate the magnets. Refinement of the magnetic drive force analysis lead to the net drive force profile plotted in Figure 66. Modifications of the profile with significantly reduced peak force values will result from the inclusion of ferromagnetic material in the region between the beds. During one complete $2 \mathrm{~s}$ cycle, the drive displaces the magnets $46 \mathrm{~cm}$ in $0.5 \mathrm{~s}$, then holds their position over the beds for $0.5 \mathrm{~s}$, and again displaces them $46 \mathrm{~cm}$ back to their starting point where they hold position for the final $0.5 \mathrm{~s}$. This low frequency limits the inertial load imposed on the drive as well as the accumulation of cycles.

The $46 \mathrm{~cm}$ drive stroke is derived from the optimum bed separation distance. This distance is based on the minimum allowable bed separation resulting in a maximum $0.5 \mathrm{~T}$ peak field in the demagnetized bed with the magnet centered over the adjacent companion bed. The magnet axial field drop off to $0.5 \mathrm{~T}$ is dependent, to a large degree, on the bore proportions which, in turn, are defined by the regenerator bed geometry.

A hydraulic system was specified with two considerations paramount. First, system flexibility is important. Cycle times, and hence, velocity and dwell, must be adjustable to map out performance envelopes. Programmable systems using servohydraulics offer this flexibility. Second, true drive forces are not yet known with high certainty. The magnetic force on the beds depends on the bed material magnetization which varies as the bed material cycles thermally. Also, the simplified magnetic analysis models the beds as a collection of several discrete, rather than distributed masses.

Mechanical systems optimized for operation at one load point and cycle are less flexible and suitable for experimental application and prototype development. The fiexibility offered by the servo-hydraulic system is considered essential in this phase of liquefier development. The proposed schematic diagram for the system is illustrated in Figure 67. This is a self-contained, turnkey system fabricated and assembled complete by the vendor. It will interface to the hydraulic cylinder on the liquefier with a pair of flexible hydraulic hoses and electrical connections for feedback from the displacement transducer integral to the cylinder. 


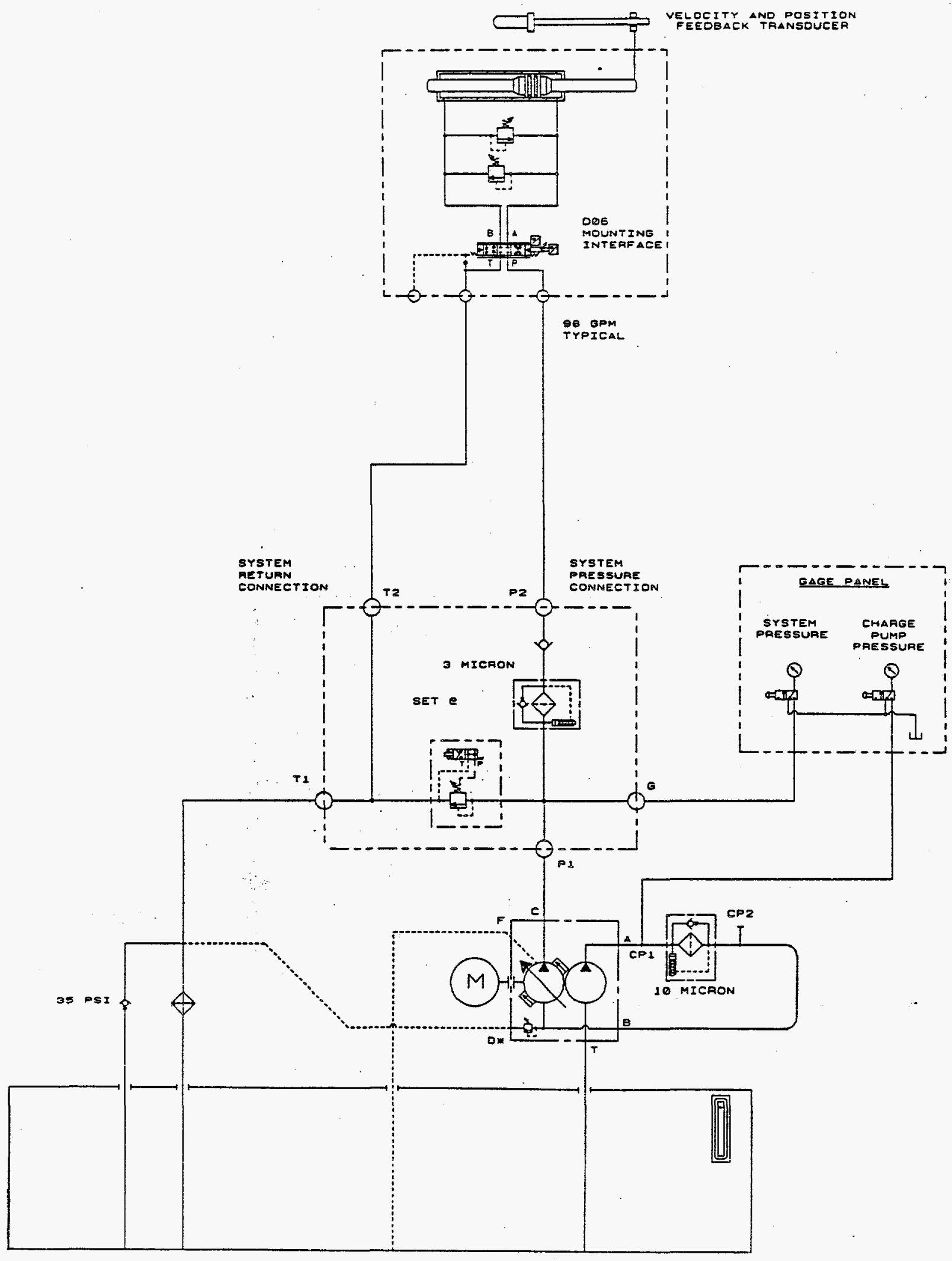

Figure 67. Programmable Servo-Hydraulic Schematic 


\subsubsection{Mechanical Linkage}

The motion generated by the hydraulic drive is transmitted through a mechanical linkage to the magnets. The actual forces transmitted are monitored by a load cell mounted at the end of the hydraulic cylinder rod. The data from this load cell coupled with position and velocity data from the displacement transducer on the cylinder are used to calculate accurately the actual mechanical refrigeration work performed by the liquefier.

The drive linkage is composed of a linear vacuum feed-through, main drive tube, and secondary drive tubes. The second stage magnet is rigidly connected to the first stage magnet by an additional set of secondary drive tubes. The entire drive system with magnets is illustrated in Figure 68. The transition from the single main drive tube to the pair of secondary drive tubes is made with a stainless steel yoke. The hydraulic cylinder mounts to the vacuum feed-through housing. Details of this subassembly are shown in Figure 69. An adaptor plate provides the mechanical interface and seal between the cylinder and feed-through housing. An elastomer bellows isolates the main vacuum vessel volume from the evacuated housing feed-through volume. This enhances bellows life and performance by reducing the pressure differential across it to zero. Housing vacuum is generated by the liquefier vacuum system roughing pump. 


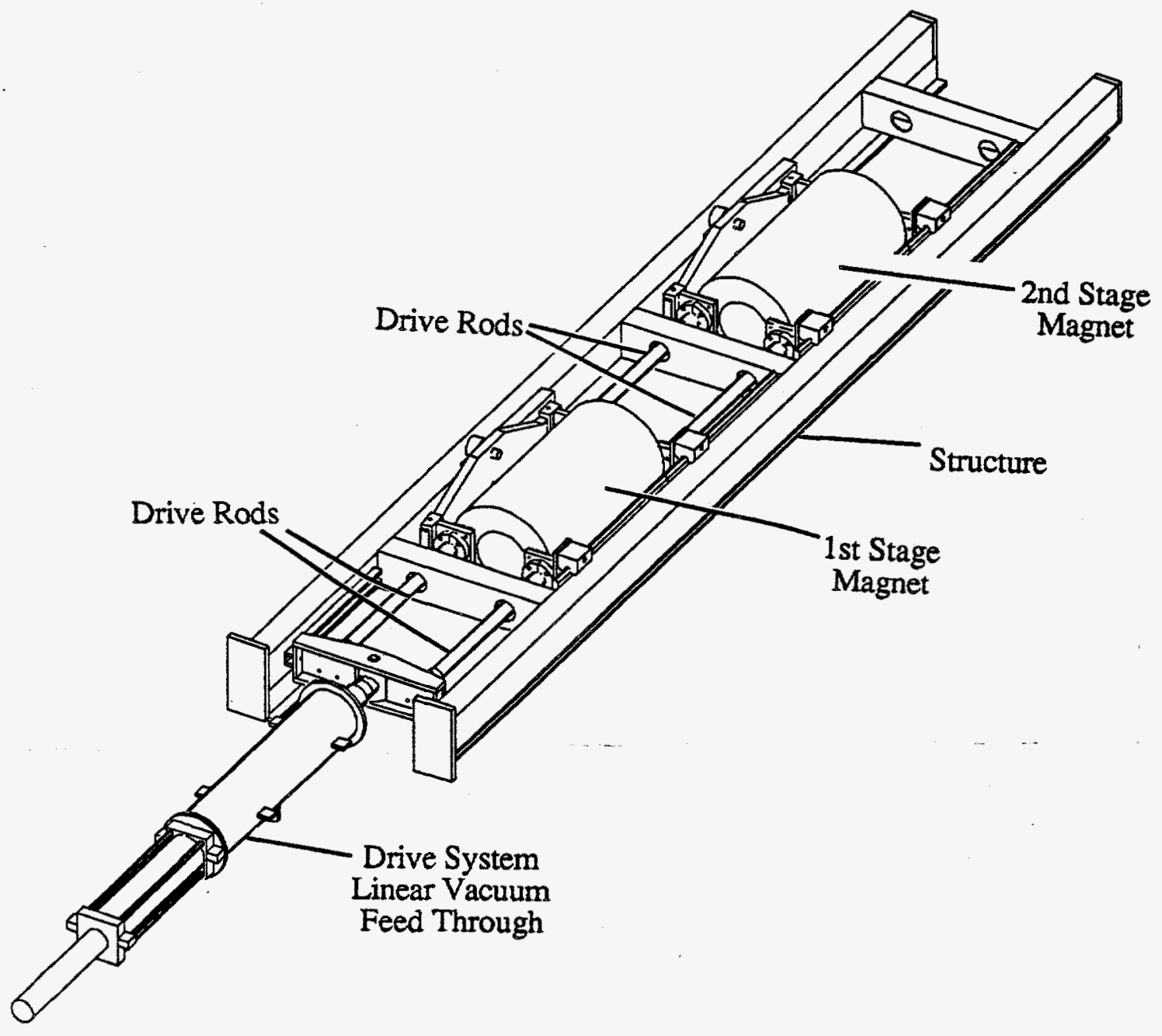

Figure 68. Drive System with Magnets 

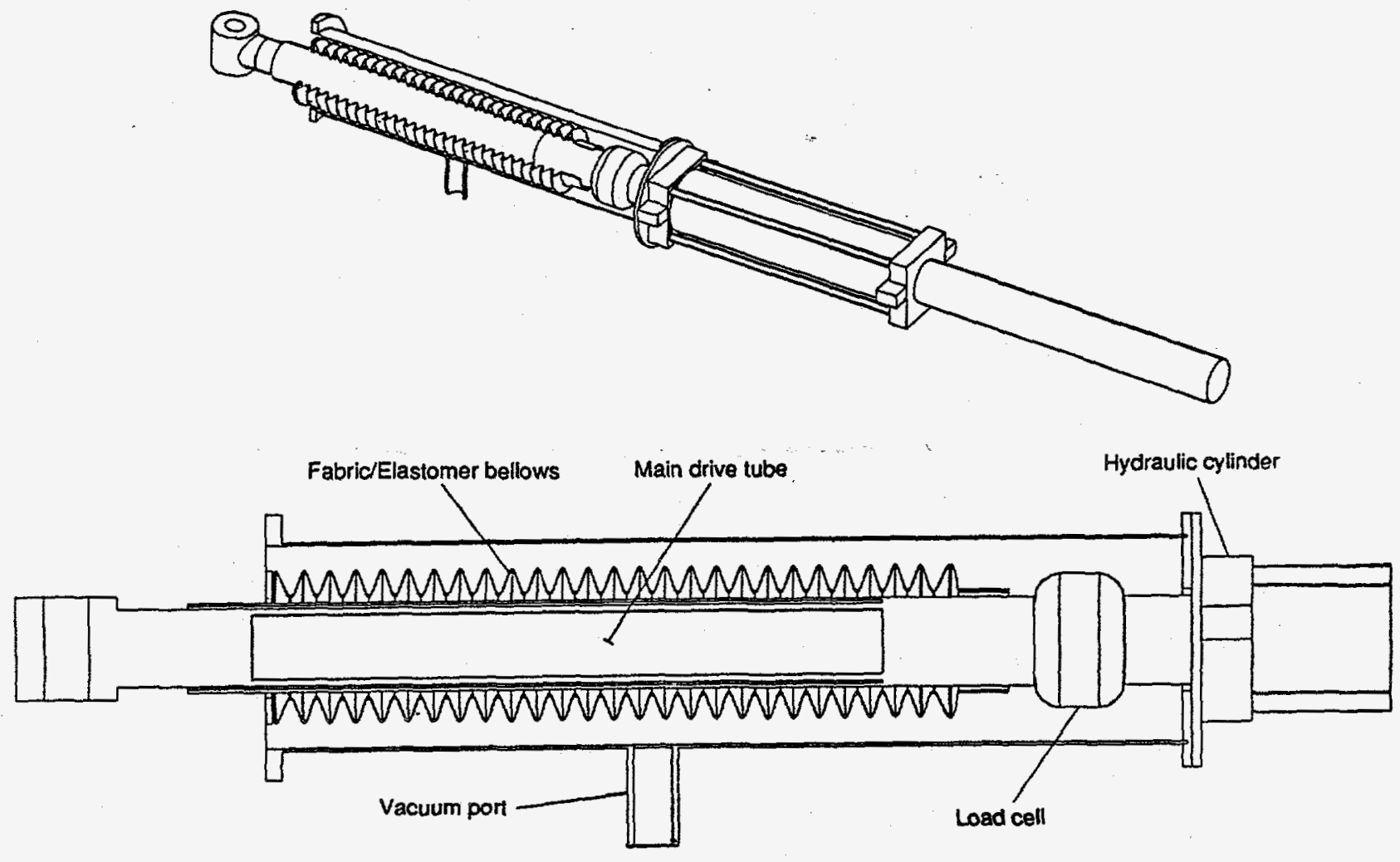

Figure 69. Drive System Linear Vacuum Feed-through 
The main drive tube is a stainless steel and composite assembly. Its composite tubular body is rigidly pinned and bonded to stainless steel ends. The tubes are fabricated from composite materials to minimize conductive heat leaks to the magnets. The threaded end fastens to the load cell and the opposite end pins to the stainless steel yoke. This pinned end is thermally anchored to the liquid nitrogen cooled support structure. A non-load carrying stainless steel tube, concentric with but thermally isolated from the composite main drive tube, is guided at the vacuum vessel wall by guide rollers. The yoke also is guided by rollers engaged in tracks mounted to the support structure beams. The pair of secondary drive tubes are rigidly fastened to the yoke and the adaptor plates secured to the front of the first stage magnet. The magnet linear guide bearings are also mounted to these plates. This simplifies the magnet/drive interface. Only a few threaded mounting holes are required on each end of each magnet. Similar adaptor plates mount to each of the remaining magnet ends. The adaptor plates are included in Figure 68. The resulting 3-point support configuration using trunnion-mounted linear bearings for guidance offers generous alignment flexibility and simplicity without expensive precision. A duplicate set of secondary drive tubes rigidly couples the facing adaptor plates between the first and second stage magnets.

The conductive heat leaks through the drive mechanism to the magnets are very low. This minimizes the helium boil-off in the magnets. The heat leak predicted by thermal analysis of the drive mechanism with SDRC software is $33 \mathrm{~mW}$. The beam element model constructed for this analysis, along with boundary conditions, is illustrated in Figure 70. Temperature dependent material properties for the stainless steel yoke and composite tubes was applied to the analysis. In the first analysis the yoke temperature was allowed to float. It equilibrated at $180 \mathrm{~K}$. Under these conditions the heat leak into the magnets is $125 \mathrm{~mW}$. When the yoke is thermally anchored to the $77 \mathrm{~K}$ support structure the heat leaked to the magnets is reduced to $33 \mathrm{~mW}$. Under these conditions $165 \mathrm{~mW}$ is intercepted at the yoke from the main drive tube. This is larger than the $125 \mathrm{~mW}$ leak because the thermal gradient in the main tube is greater in the second case with the yoke at $77 \mathrm{~K}$ rather than $180 \mathrm{~K}$. Heat fluxes throughout the drive mechanism are plotted in Figure 71 . Interface conductances have been neglected to simplify this analysis. Accounting for these conductances would reduce further the estimates for heat leaks into the magnets.

The structural analysis of the drive mechanism is coupled to the thermal analysis. Small tube sections are required to minimize the conductive heat leaks but conflict with strength considerations. The issue is further complicated by the cyclic, reverse loading on the tubes. A small cross section fails in buckling while a large cross section conducts too much heat. The analysis attempted to minimize area, maximize length, and minimize $1 / \mathrm{r}$ ratios. A 40000 pound load applied to optimization runs with stress constraints set to 20000 psi yielded 26 in long, 2.0 in O.D. secondary drive tubes and a 34 in long, 3.0 in O.D. main drive tube. 
2" 0.D. $\times .164$ wall $\times 6$ 6" 39.

G10 rube

(2 Places)

gid

s.s. 8ar

3" 0.0. $\times .215$ Wall $\times$ 6" 19 .

3" 0.0.

G10 Tube
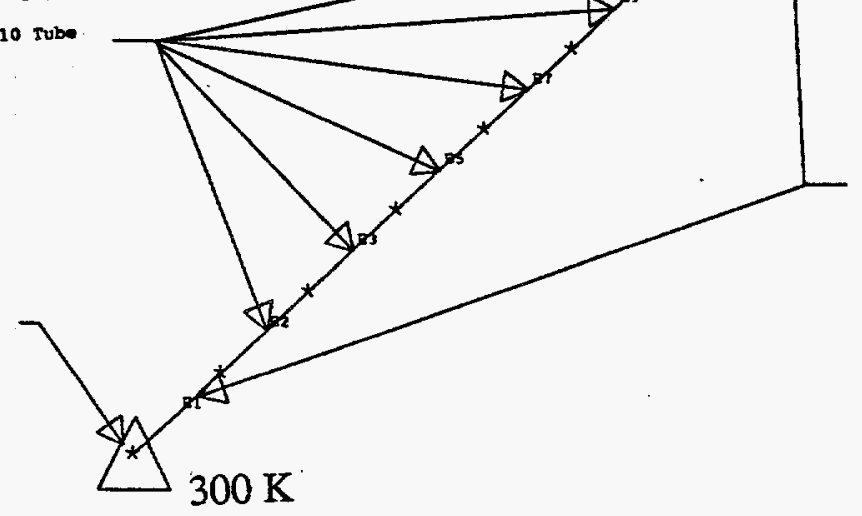

$\AA^{4 K}$

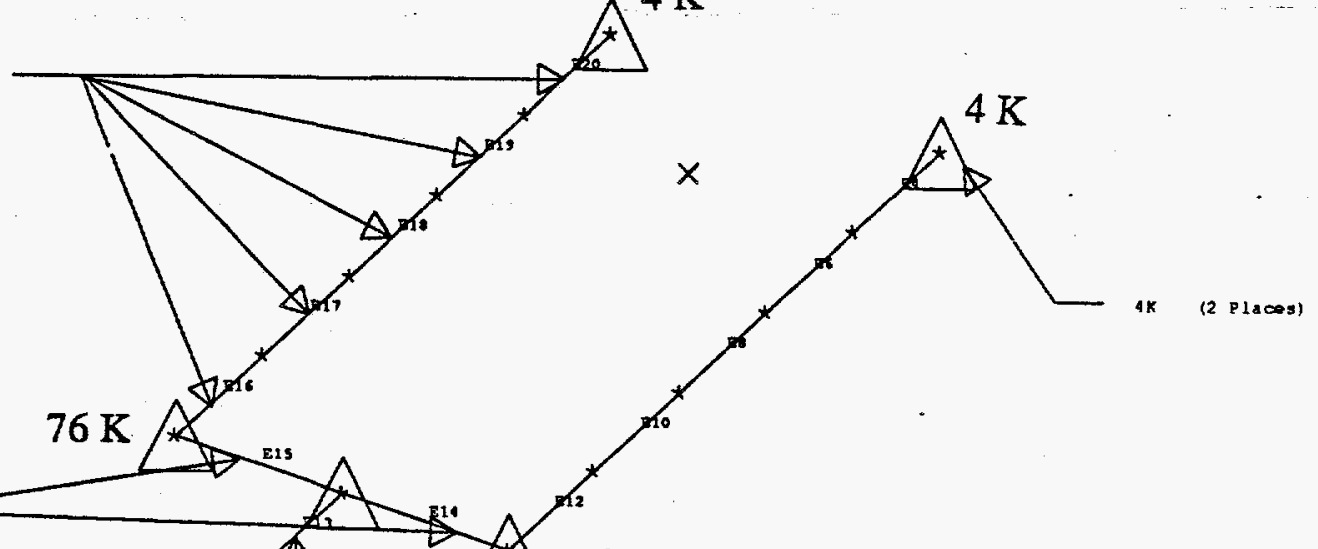

3" 0.0. $\times 6=19$.

s.s. Rod

$76 \mathrm{~K}$

(3 Pl nces)

Figure 70. Drive Mechanism Thermal Analysis Model 
Heat Flux for Unit Area

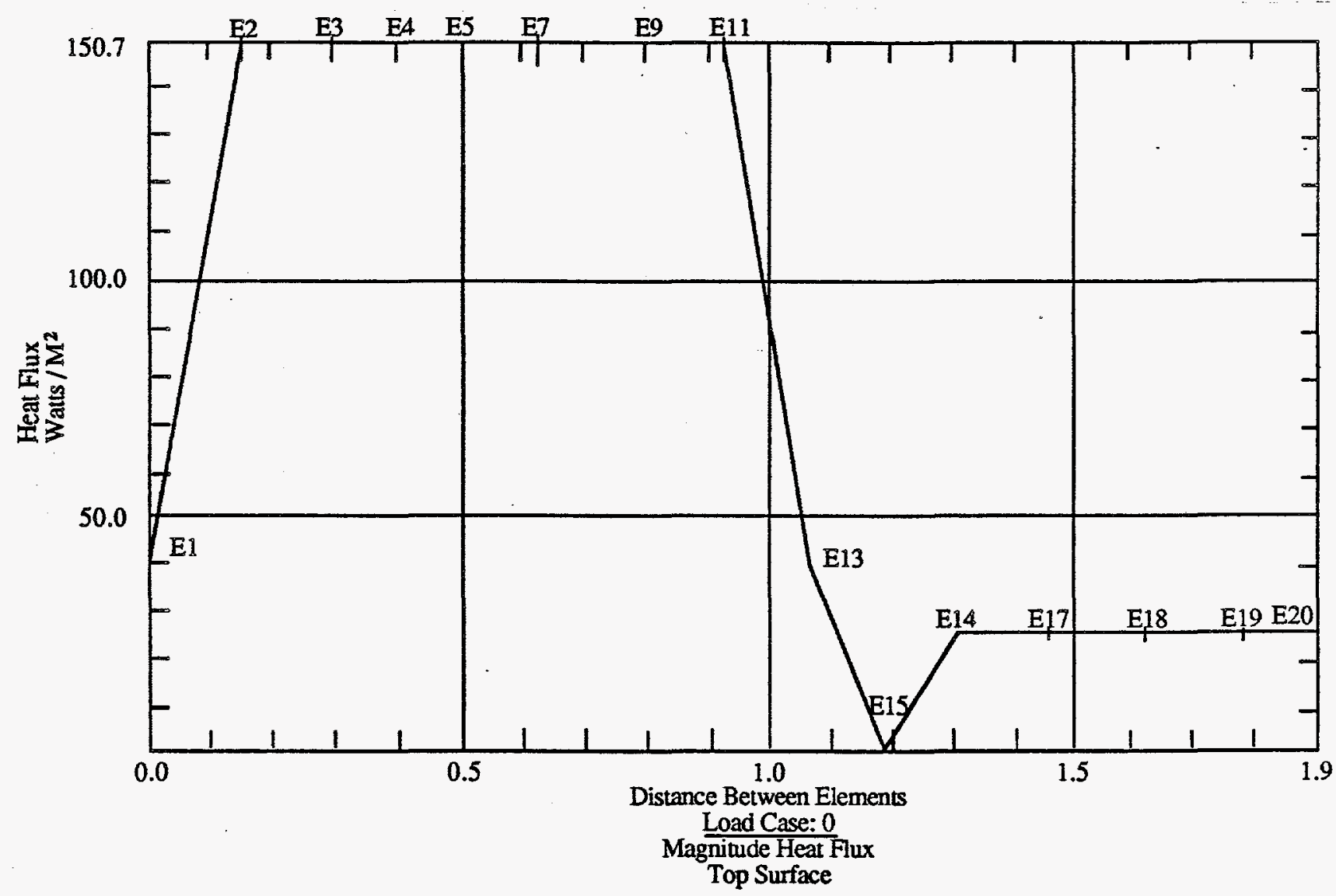

Figure 71. Heat Fluxes throughout the Drive Mechanism (B) 
The drive yoke was modeled extensively, particularly at the main drive tube pin joint. Several different trial load distributions were evaluated to correctly model the interaction between the pin and yoke. Typically, pinned joint design is straightforward, given adequate safety factors. In this case, minimum bearing areas are desired to again minimize heat conduction through the drive.

\subsection{Vacuum System}

A vacuum system selection capable of establishing a vacuum of $10^{-7}$ torr in the liquefier vacuum chamber in 24 hours has been identified. The vacuum pumping system includes the turbomolecular pump, backing pump for the turbomolecular pump, separate roughing pump, ion gauge control with ion gauge tube, two thermocouple gauges and process relays, two vane pump exhaust demisters, 10" American Standards Association (ASA) electropneumatic valves, foreline valve, rough valve, NEMA-12 control cabinet with motor starters for the vane pump, and valve controls/indicators. A schematic of the system is shown in Figure 72 . The gate and roughing valves are electrically interlocked to prevent opening simultaneously. The valves are also interlocked with process set point of the gauge control. All joints are TIG welded.

The turbo pump is a Leybold model TMP-1000 air or liquid-cooled unit that can pump down to $10^{-10} \mathrm{mbar}$ (rated $1150 \mathrm{l} / \mathrm{s}$ - air). The fore pump which backs up the turbo pump is a Leybold model D-60A, dual-stage, direct drive, air-cooled vane pump that pumps at $36.7 \mathrm{cfm}$ down to $10^{-2}$ mbar.

The roughing pump is a Leybold pump model D-90A, dual-stage, direct drive, air-cooled vane pump that pumps at $55 \mathrm{cfm}$. This system will pump down the chamber to $10^{-5} \mathrm{mbar}$ in about four hours and the turbo will get down to $10^{-7} \mathrm{mbar}$ in about (20) hours, and will run continuously to maintain $10^{-7}$. There is a great deal of surface area to pump down so that predicted time may vary slightly. The system will be protected from a large differential pressure increase by the electrically-operated gate valve located at the interface to the vacuum chamber. The total power requirement is a three-phase, $60 \mathrm{~Hz}, 208 / 230 / 460$ system rated at about $10 \mathrm{H}$. P.

The above vacuum pumping system represents a production model where a short pump downtime is not a requirement. However, for a developmental prototype a larger roughing pump will be required to pump down accessories and speed up evacuation of the chambers. Flushing the system and chamber with an inert gas will also reduce the pump down time.

The vacuum chamber is made of two large sections: a rectangular section with a 1" plate $\times 3^{\prime}-0^{\prime \prime} \times 4-0^{\prime \prime} \times 7^{\prime}-0^{\prime \prime}$ and a $3 / 8^{\prime \prime}$ thick $45^{\prime \prime}$ I.D. x $12^{\prime}-4^{\prime \prime}$ long cylinder. The cylinder is mounted on rails with $6^{\prime \prime}$ diameter nonswivel casters to permit smooth and accurate alignment when servicing the liquefier assembly. All appendages, outlets, 


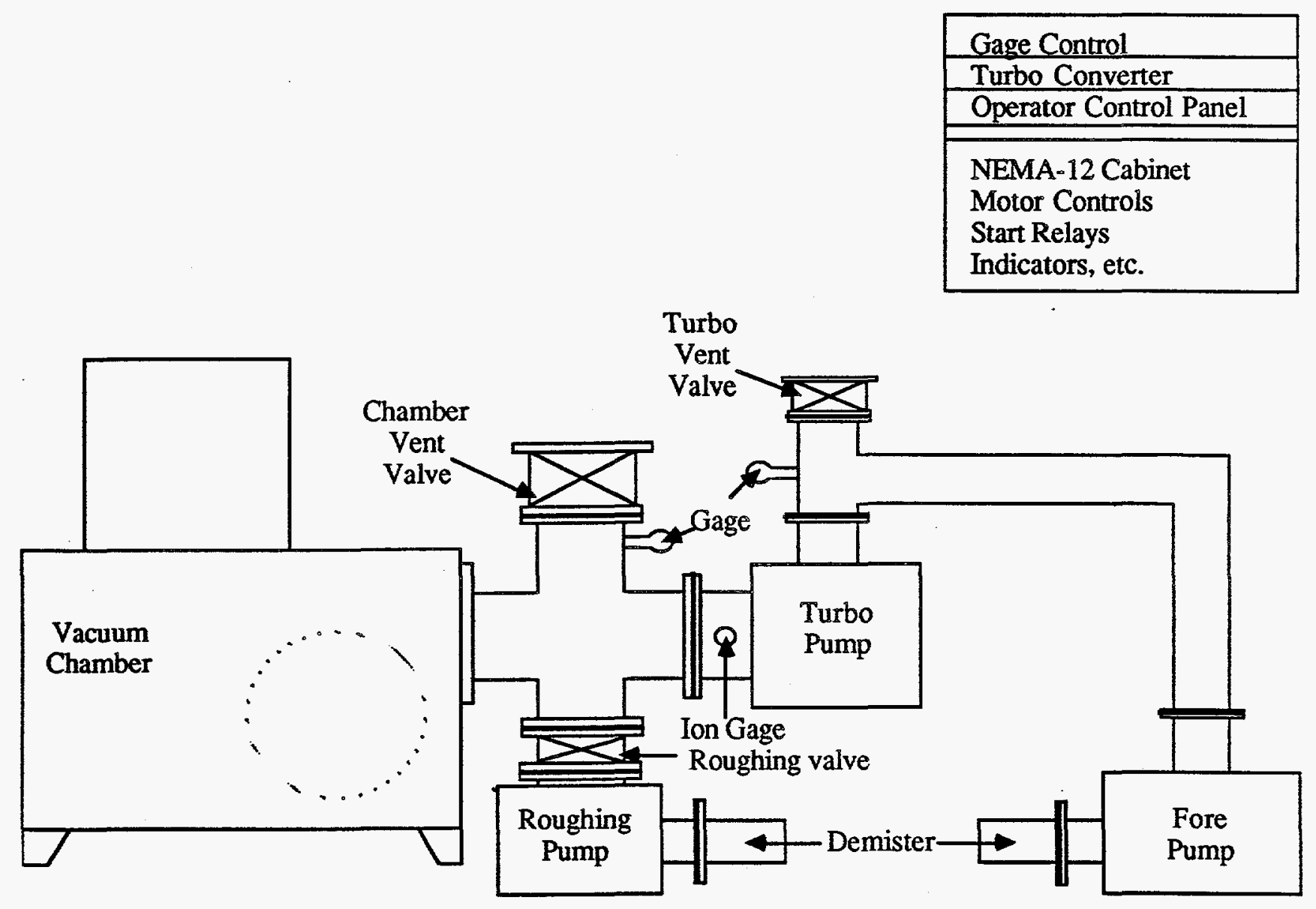

Figure 72. Simplified Vacuum System Schematic 
inlets, valves, and pump will be located in the rectangular section. All welds will be ground and polished. Flat sections will be reinforced as required. All flanged connections will be finished to 16 microinch or better. Steel thread inserts will be used for bolted connections.

\subsection{Piping System}

The liquefier helium circuit generates the flows necessary to produce sufficient cooling for counterflow heat exchange with the hydrogen process stream. The helium flow is intermittent and the hydrogen stream is continuous. The heat capacity of the counterflow heat exchanger is large enough to limit any temperature fluctuation in the hydrogen process stream. The bidirectional nature of helium flow through the regenerator beds necessitates a valving and control arrangement to coordinate the "blow" and dwell periods of the AMR cycle with the magnet motion generated by the drive. The simple, pictorial flow schematic in Figure 56 includes all major system components. These include heat exchangers, directional and proportioning valves, flow meters, circulation pump, and beds. The pump provides a continuous flow of $2 \mathrm{MPa}$ (20 atm) helium gas through the refrigerator with up to $0.14 \mathrm{MPa}$ (20 psi) boost. This accounts for pressure drops throughout the system. The actual system pressure drop is estimated to be $0.10 \mathrm{MPa}(15 \mathrm{psi})$. The beds account for approximately half of the total flow restriction with the remainder distributed among the valves, heat exchangers, flow meters, and piping. An isometric view of the piping system showing all major components is illustrated in Figure 74.

During dwell periods when the magnets are in motion $(0.5 \mathrm{~s})$, the valves are positioned such that all helium flow is diverted directly to the pump inlet (position 2 in the schematic, Figure 56), thereby bypassing the refrigerator. During "blow" periods when the magnets are stationary $(0.5 \mathrm{~s})$, the valves shift to direct helium flow through the refrigerator. The $2 \mathrm{~s}$ cycle consists of two dwell and two blow periods. During a blow period, a $436 \mathrm{~g} / \mathrm{s}$ mass flow passes through the demagnetized first stage bed and splits. A portion of the flow passes on to the second stage demagnetized bed while the remainder removes heat from the magnetized first stage bed and returns to the pump. Flow leaving the demagnetized second stage bed at about $19 \mathrm{~K}$ is split between the ortho-para converters and hydrogen-helium heat exchanger (O/P Heat Exchanger) and the magnetized second stage bed. The mass flow rates through the $O / P$ Heat Exchanger and the bed are $10 \mathrm{~g} / \mathrm{s}$ and $77 \mathrm{~g} / \mathrm{s}$, respectively. These flows also return to the pump. The flow is then diverted during the $0.5 \mathrm{~s}$ dwell period while the magnets shuttle to the previously demagnetized beds. Flow is then resumed in the opposite direction for another $0.5 \mathrm{~s}$ blow and then diverted while the magnets shuttle back to the originally magnetized beds.

The flow diverted to the O/P Heat Exchanger not only cools the hydrogen gas to $20 \mathrm{~K}$, but also removes the heat of conversion from ortho-to-parahydrogen in a typical liquefier. The relative equilibrium concentrations of these states varies with 


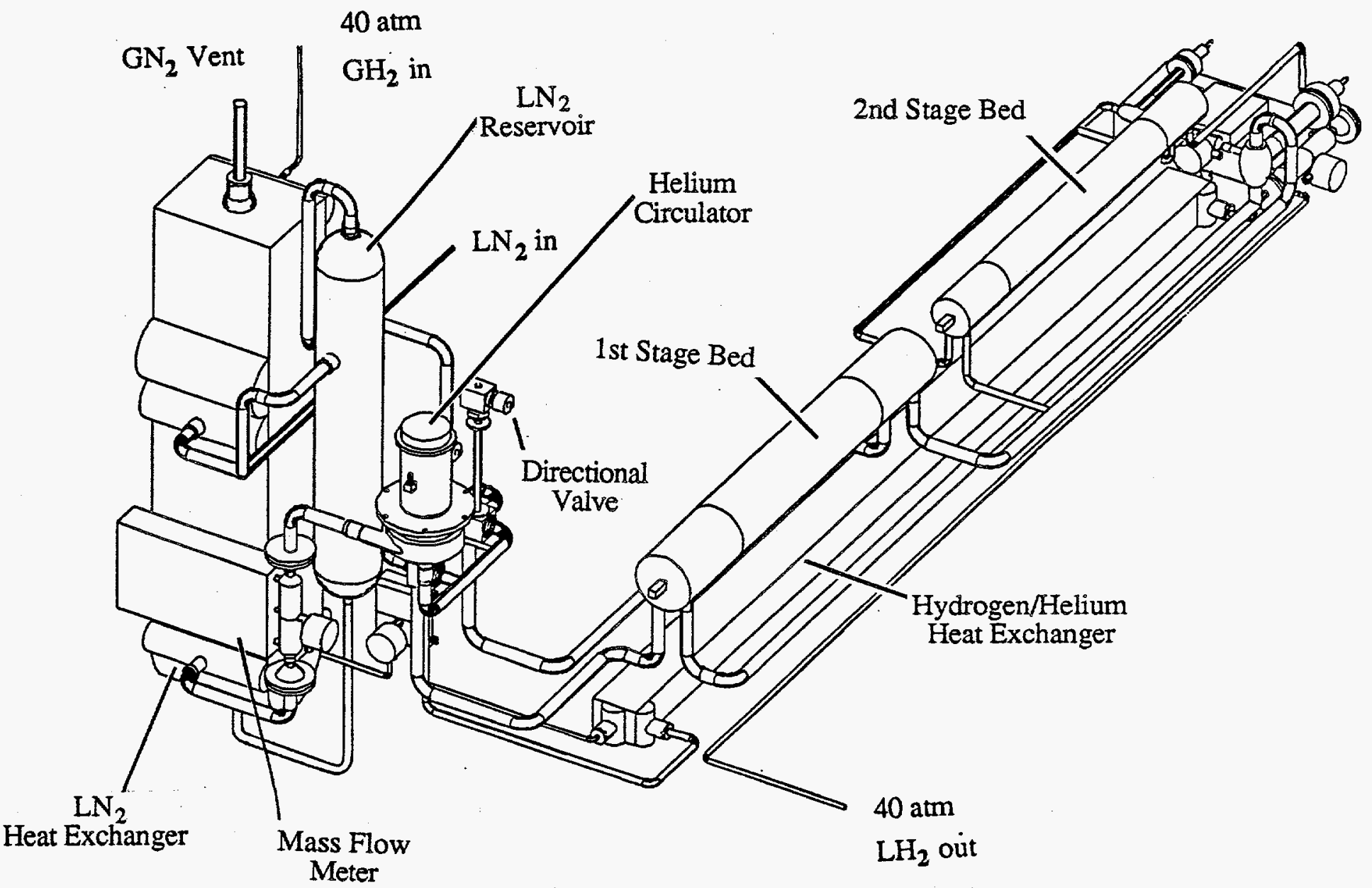

Figure 73. Isometric View of Piping System Assembly 
temperature as shown in Figure 74. Conversion of orthohydrogen to parahydrogen is a naturally occurring exothermic reaction. Room temperature hydrogen gas liquefied without ortho-to-para conversion will boil off at an initial rate of approximately $20 \%$ per day as it naturally converts to $100 \%$ parahydrogen. Optimum ortho-to-para conversion is accomplished in incremental stages to remove the associated heats of conversion at the highest possible temperatures. These incremental stages for the liquefier are superimposed on the figure. Expensive catalysts are employed in this process. To demonstrate a complete liquefaction system, the liquefier simulates the production of $100 \%$ parahydrogen by integrating electric heaters in place of ortho-to-para catalytic reactors into the O/P Heat Exchanger. The heats of conversion are simulated by the heaters in place of the more costly catalysts. Catalytic reactors could be retrofitted to the O/P Heat Exchanger to produce approximately $100 \%$ parahydrogen.

The O/P Heat Exchanger simulates the heats of conversion in four stages while incrementally cooling the hydrogen before and after each stage. The hydrogen process stream is diverted from the heat exchanger for each separate ortho-to-para conversion simulation. Reentrance of the heated hydrogen gas occurs at a location of roughly equivalent temperature in the heat exchanger. A relatively linear temperature gradient spans its 132 inch length.

Eddy current heating in this brazed aluminum heat exchanger is estimated to be roughly $15 \mathrm{~W}$ in a $0.1 \mathrm{~T}$ stray field. This value is small relative to the total cooling available from the helium flow. The primary source of this heating is the 0.25 inch outer wall. The narrow serrated fins and parting sheets generate little heat due to the very small current loops generated in their thickness.

The $\mathrm{LN}_{2}$ heat exchanger precools the hydrogen process stream prior to entering the O/P Heat Exchanger. It also precools the circulating helium gas after it exits the circulation pump to remove the heat of compression. An $\mathrm{LN}_{2}$ reservoir is coupled to the precooler heat exchanger to continuously replenish the liquid that boils off within the precooler during heat exchange with the helium and hydrogen streams. Boiling heat exchange takes place in the bottom zone of the vertically-oriented assembly at a rate of approximately $169 \mathrm{~kg} / \mathrm{hr}(370 \mathrm{lbs} / \mathrm{hr})$. Cold nitrogen boil-off vapor provides initial cooling to the hydrogen flow in the top zone of the heat exchanger prior to being vented. The flow connections and basic outline of the $\mathrm{LN}_{2}$ heat exchanger are illustrated in Figure 75. Like the O/P Heat Exchanger it is a brazed aluminum fabrication. 

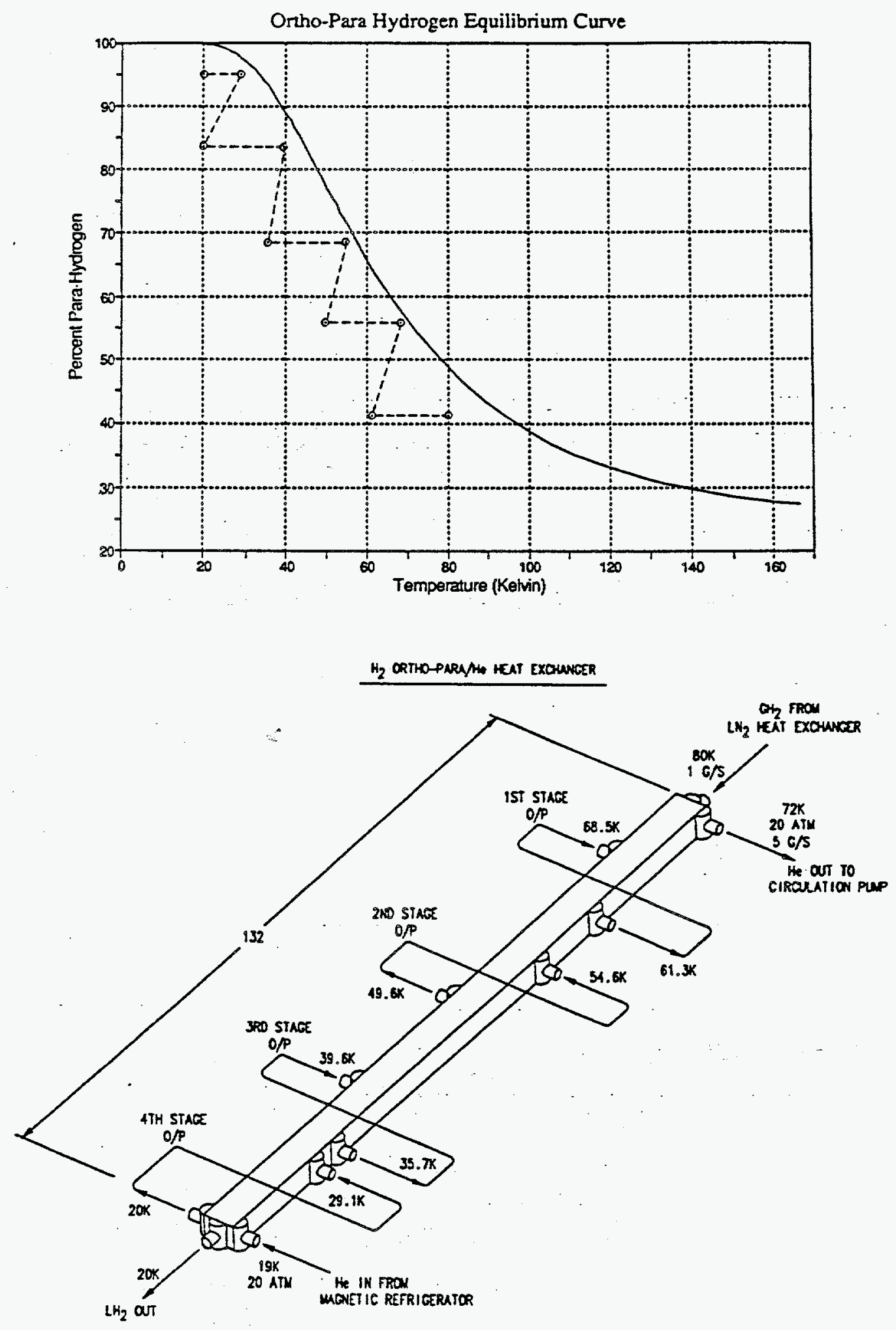

Figure 74. Ortho-Para Conversion Increments 


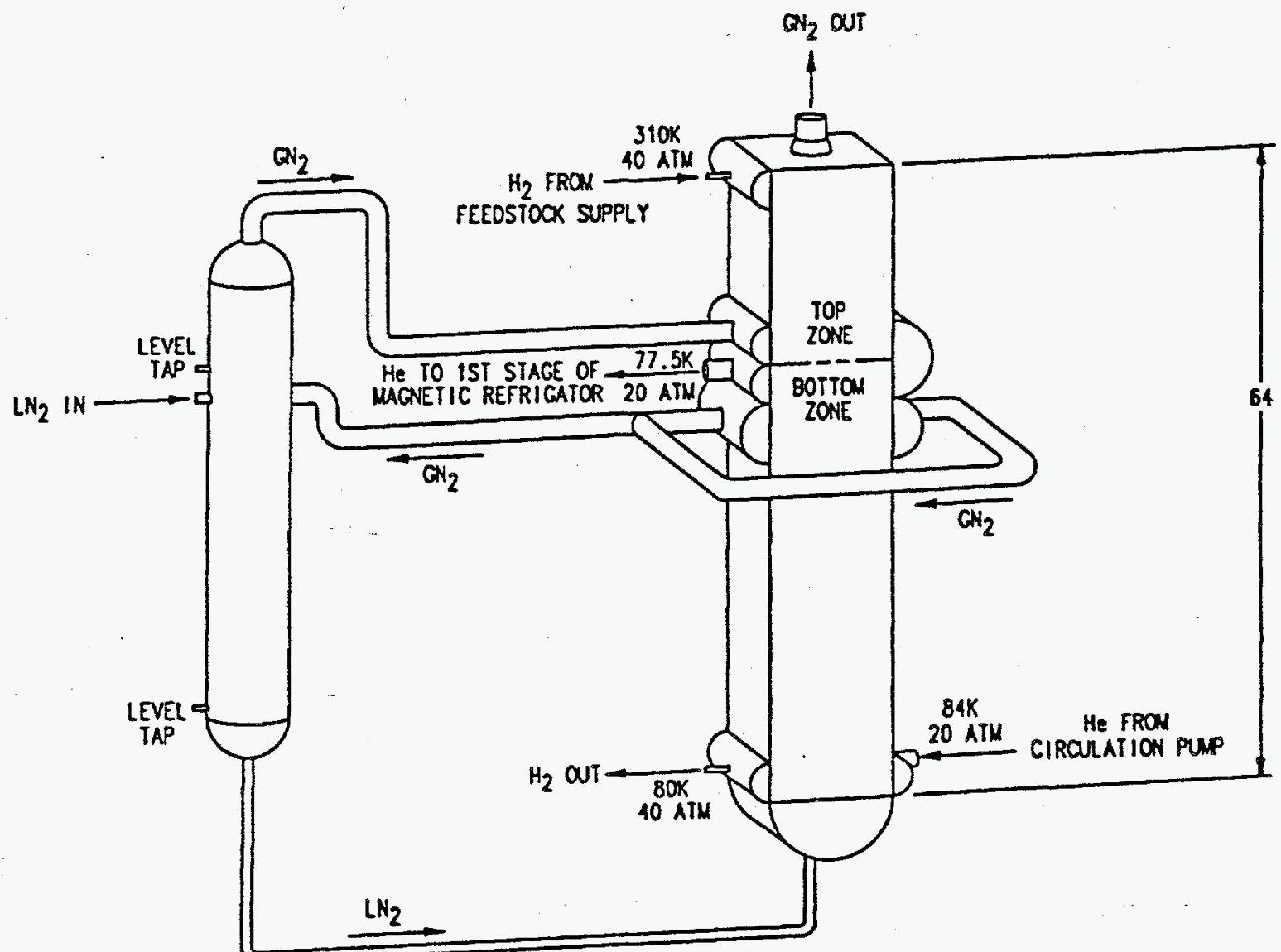

Figure 75. $\mathrm{LN}_{2}$ Heat Exchanger Piping Connections 
The helium flow out of the $\mathrm{LN}_{2}$ heat exchanger passes through the directional valves and is proportioned throughout the remainder of the piping system. The control system continuously monitors the helium flow rates through the pump, second stage beds, and O/P Heat Exchanger during operation. Necessary adjustments to the flow proportioning between first and second stages can be made with the flow proportioning valves. These valves are located at points in the flow circuit where the bidirectional flows are the same in both directions. Flow measurement is based on the coriolis effect by passing all fluid measured through a resonating sensor tube. The sensor tube frequency varies with mass flow rate. Meter accuracy and pressure drop are exceptional. Accuracy is within $1 \%$ and pressure drops are $6.8 \times 10^{-3} \mathrm{MPa}(1 \mathrm{psi})$ or less. A flow meter on the hydrogen line is used to provide a measure of the maximum liquefaction rate possible assuming no boil-off losses or two-phase hydrogen flow out of the $\mathrm{O} / \mathrm{P}$ Heat Exchanger. The meter also is necessary to set the appropriate flow balance between the helium and hydrogen streams. The meters offer the capability to not only measure instantaneous mass flow rates but also cumulative mass flow over time. This is a valuable feature for performance validation testing.

The control system also actuates the three-way directional fiow control valves used to reverse the helium flows. These fast acting cryogenic ball valves are pneumatically operated with $10.3 \mathrm{MPa}(1500 \mathrm{psi})$ nitrogen. Their response time is roughly $0.25 \mathrm{~s}$ at this pilot pressure. Higher pressures will decrease response time. The nitrogen compressor can produce pressures as high as $34.4 \mathrm{MPa}(5000 \mathrm{psi})$ if quicker response times are necessary.

The helium circulator is a constant speed, centrifugal cryogenic pump operating at $12,400 \mathrm{rpm}$ and $55 \%$ efficiency. Its hermetic design eliminates helium contamination. Cryogenic rolling element bearings allow for cold operation. The impeller is isolated from the motor housing by an extension to minimize parasitic heat loads. A variable frequency drive controls the pump speed and helium fiow rate. A very broad range of flow rates is available for mapping out the liquefier performance envelope.

All piping system components including valves, flow meters, pump, heat exchangers, and beds are interconnected with stainless steel tubing. All first stage tubing is $38.1 \mathrm{~mm}$ (1.5 in. O.D.) diameter with $1.65 \mathrm{~mm}(0.065 \mathrm{in}$.) wall thickness. All second stage tubing is $12.7 \mathrm{~mm}(0.5 \mathrm{in}$. O.D.) diameter with $0.71 \mathrm{~mm}(0.028 \mathrm{in}$.) wall thickness. The hydrogen process stream line is $6.35 \mathrm{~mm}(0.25 \mathrm{in}$.) diameter with 0.71 $\mathrm{mm}(0.028 \mathrm{in}$.) wall also. All joints throughout the system are welded with a high quality tube welder to minimize the heat-affected zone in the welds and generate consistently leak-tight joints. The bolted joints between the beds and tubing use C-ring seals captured between the tube flange and an embedded stainless steel ring bonded to the bed assembly. This joint design decouples the structural and sealing functions. The bolts and flange carry the structural loads on the tubing while the unstressed, embedded ring makes the seal with the $\mathrm{C}$-ring. This joint design can be retrofitted to a permanent, bonded joint if necessary later on. 
Initial concerns of excessive eddy current heating in the piping lead to the use of G-10 tubing throughout the system. Successful stray flux containment around the magnets, however, alleviates this concern. Consequently, estimated eddy current heating in stainless steel tubing near the magnets is marginal. Stainless steel tubing is used throughout the system and offers higher reliability and joint integrity over the G10 .

\subsection{Prototype Instrumentation and Control}

The prototype instrumentation and control design is divided into three main areas 1) Sensor type and locations; 2) Control logic and operational flexibility; and 3) Anticipated operating parameters.

\subsection{Sensor Type and Locations}

The liquefier will be instrumented with more than 100 sensors. Included in Table 8 is a preliminary list of sensor locations and type.

Table 8. Sensor Location and Type

\begin{tabular}{|ll|}
\hline Sensor Location & Sensor Type \\
& \\
1st stage He regen \#1 warm temperature & Bizmuth Ruthenium Oxide Resistor \\
1st stage He regen \#1 cold temperature & Bizmuth Ruthenium Oxide Resistor \\
1st stage He regen \#2 warm temperature & Bizmuth Ruthenium Oxide Resistor \\
1st stage He regen \#2 cold temperature & Bizmuth Ruthenium Oxide Resistor \\
2nd stage He regen \#3 warm temperature & Bizmuth Ruthenium Oxide Resistor \\
2nd stage He regen \#3 cold temperature & Bizmuth Ruthenium Oxide Resistor \\
2nd stage He regen \#4 warm temperature & Bizmuth Ruthenium Oxide Resistor \\
2nd stage He regen \#4 cold temperature & Bizmuth Ruthenium Oxide Resistor \\
He O/P \#1 inlet temperature & Bizmuth Ruthenium Oxide Resistor \\
He O/P \#2 temperature & Bizmuth Ruthenium Oxide Resistor \\
He O/P \#3 temperature & Bizmuth Ruthenium Oxide Resistor \\
He O/P \#4 temperature & Bizmuth Ruthenium Oxide Resistor \\
He O/P \#5 outlet temperature & Bizmuth Ruthenium Oxide Resistor \\
He pump inlet temperature & Bizmuth Ruthenium Oxide Resistor \\
He pump outlet temperature & Bizmuth Ruthenium Oxide Resistor \\
He LN2 HEX inlet temperature & Bizmuth Ruthenium Oxide Resistor \\
He LN2 HEX outlet temperature & Bizmuth Ruthenium Oxide Resistor \\
\hline
\end{tabular}


Table 8. Sensor Location and Type (continued)

\begin{tabular}{|c|c|}
\hline Sensor Location & Sensor Type \\
\hline H2 LN2 HEX inlet temperature & Bizmuth Ruthenium Oxide Resistor \\
\hline H2 LN2 HEX outlet temperature & Bizmuth Ruthenium Oxide Resistor \\
\hline $\mathrm{H} 2 \mathrm{O} / \mathrm{P} \# 5$ inlet temperature & Bizmuth Ruthenium Oxide Resistor \\
\hline $\mathrm{H} 2 \mathrm{O} / \mathrm{P} \# 4$ temperature & Bizmuth Ruthenium Oxide Resistor \\
\hline $\mathrm{H} 32 \mathrm{O} / \mathrm{P} \# 3$ temperature & Bizmuth Ruthenium Oxide Resistor \\
\hline $\mathrm{H} 2 \mathrm{O} / \mathrm{P} \# 2$ temperature & Bizmuth Ruthenium Oxide Resistor \\
\hline $\mathrm{H} 2 \mathrm{O} / \mathrm{P} \# 1$ outlet temperature & Bizmuth Ruthenium Oxide Resistor \\
\hline $\mathrm{H} 2$ storage tank temperature & Bizmuth Ruthenium Oxide Resistor \\
\hline Magnet demountable lead temperature & Carbon Glass Resistor \\
\hline Magnet demountable lead temperature & Carbon Glass Resistor \\
\hline Magnet \#1 temperature & Carbon Glass Resistor \\
\hline Magnet \#2 temperature & Carbon Glass Resistor \\
\hline Persistent mode switch temperature & Carbon Glass Resistor \\
\hline $\mathrm{H} 2$ flow rate between $\mathrm{HEX}$ & Micromotion DS012S Coriolis \\
\hline He flow rate after blower & Micromotion DS150S Coriolis \\
\hline He flow rate 2 nd stage into $\mathrm{O} / \mathrm{P} \mathrm{HEX}$ & Micromotion DS025S Coriolis \\
\hline He 2 nd stage flow rate & Micromotion DS100S Coriolis \\
\hline He blower inlet pressure & Keller PSI Series 400 Cryogenic \\
\hline He blower outlet pressure & Keller PSI Series 400 Cryogenic \\
\hline 1st stage He regen \#1 warm pressure & Keller PSI Series 400 Cryogenic \\
\hline 1st stage He regen \#1 cold pressure & Keller PSI Series 400 Cryogenic \\
\hline 1st stage He regen \#2 warm pressure & Keller PSI Series 400 Cryogenic \\
\hline 1st stage $\mathrm{He}$ regen $\# 2$ cold pressure & Keller PSI Series 400 Cryogenic \\
\hline 2nd stage He regen \#3 warm pressure & Keller PSI Series 400 Cryogenic \\
\hline 2nd stage He regen \#3 cold pressure & Keller PSI Series 400 Cryogenic \\
\hline 2nd stage He regen \#4 warm pressure & Keller PSI Series 400 Cryogenic \\
\hline 2nd stage He regen $\# 4$ cold pressure & Keller PSI Series 400 Cryogenic \\
\hline $\mathrm{He} \mathrm{O} / \mathrm{P} \mathrm{HEX}$ inlet pressure & Keller PSI Series 400 Cryogenic \\
\hline He O/P HEX outlet pressure & Keller PSI Series 400 Cryogenic \\
\hline $\mathrm{He}$ O/P LN2 HEX inlet pressure & Keller PSI Series 400 \\
\hline He O/P LN2 HEX outlet pressure & Keller PSI Series 400 Cryogenic \\
\hline H2 LN2 inlet pressure & Keller PSI Series 400 Cryogenic \\
\hline H2 LN2 outlet pressure & Keller PSI Series 400 Cryogenic \\
\hline $\mathrm{H} 2 \mathrm{O} / \mathrm{P}$ HEX inlet pressure & Keller PSI Series 400 Cryogenic \\
\hline $\mathrm{H} 2$ O/P HEX outlet pressure & Keller PSI Series 400 Cryogenic \\
\hline
\end{tabular}


Table 8. Sensor Location and Type(continued)

\begin{tabular}{|ll|}
\hline Sensor Location & Sensor Type \\
O/P \#1 heater voltage & N/A \\
O/P \#2 heater voltage & N/A \\
O/P \#3 heater voltage & N/A \\
O/P \#4 heater voltage & N/A \\
O/P \#1 heater current & Shunt \\
O/P \#2 heater current & Shunt \\
O/P \#3 heater current & Shunt \\
O/P \#4 heater current & Shunt \\
& \\
Magnet current & Shunt \\
& \\
Load cell on magnet drive & Eaton/Lebow \\
& \\
LN2 inside dewar depth sensor & Keller PSI Series 400 Cryogenic Liquid Level Sensor \\
LHe storage tank depth sensor & Keller PSI Series 400 Cryogenic Liquid Level Sensor \\
LH2 storage tank depth sensor & Keller PSI Series 400 Cryogenic Liquid Level Sensor \\
LHe magnet \#1 depth sensor & Keller PSI Series 400 Cryogenic Liquid Level Sensor \\
LHe magnet \#2 depth sensor & Keller PSI Series 400 Cryogenic Liquid Level Sensor \\
& \\
He blower power & 8.5 kW TBD \\
& F.W. Bell Hall Probe \\
Magnetic field \#1 regen & F.W. Bell Hall Probe \\
Magnetic field \#2 regen & F.W. Bell Hall Probe \\
Magnetic field \#3 regen & F.W. Bell Hall Probe \\
Magnetic field \#4 regen & Magnetostrictive Device \\
Magnet position & Magnetostrictive Device \\
Magnet velocity & Magnetostrictive Device \\
Magnet acceleration &
\end{tabular}

\subsection{Control Logic and Operational Flexibility}

The control logic for the liquefier is based on a modified hierarchical (multi-level) control strategy. The modifications consist of decentralized controllers coupled to the hierarchical structure via only data transfer to the decentralized controllers. The modified hierarchical control strategy structure is shown in Figure 76. 


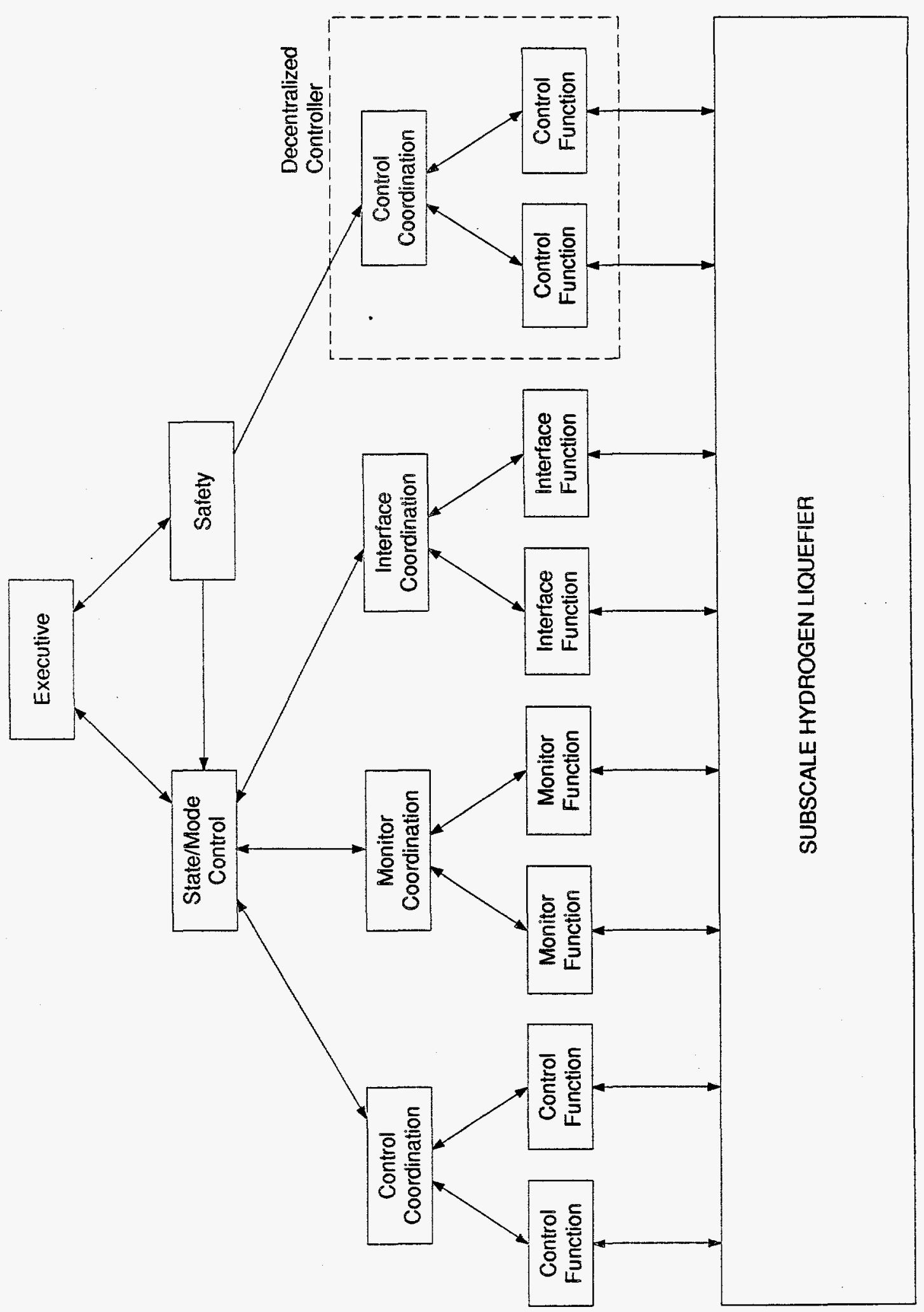

울 
Features of the modified hierarchical control strategy include:

- The modified hierarchical system consists of decision-making components structured in a pyramid shape.

- The system has an overall goal which may (or may not) be in harmony with all its individual components.

- The various levels of hierarchy in the system exchange information (usually vertically) among themselves iteratively.

- As the level of hierarchy goes up, the time horizon increases; i.e. the lower level components are faster than the higher-level ones.

- Decentralized components of the system maintain nearly all local decision making capability while transferring data to/from higher levels of the structure. The only decision made by the hierarchical control system, for the decentralized controllers, shall be a safety disable/enable decision.

\subsection{Control Logic Segregation}

The control logic is segregated into numerous control loops. Each control loop is placed at a particular level of the hierarchical system and may be implemented with multiple lower-level control loops. The set of basic control loops includes the control loops described in Table 9. 
Table 9. Control Loop Descriptions

Control Loop

Executive

Safety

Cryogen level

Display/Input

Data Storage

$\mathrm{H}_{2}$ Temperature

Catalyst Temperature \#1

Catalyst Temperature \#2

Catalyst Temperature \#3

Catalyst Temperature \#4

Data Acquisition

$\mathrm{H}_{2}$ Flow

Magnetic Drive

Bed \#1 Temperature

Bed \#2 Temperature
Description

Level

Supreme coordinator of the system

Safety control loops and warnings/alarms/cautions

4

Monitors the levels of all cryogen liquids

Graphical User Interface

Stores data acquired and control system

interim \& output values

Controls the $\mathrm{H}_{2}$ temperature

Controls the temperature of the $61.3 \mathrm{~K}$

to $68.5 \mathrm{~K}$ para- $\mathrm{H}$ catalyst simulator

Controls the temperature of the $49.6 \mathrm{~K}$

to $54.6 \mathrm{~K}$ para- $\mathrm{H}$ catalyst simulator

to $39.6 \mathrm{~K}$ para- $\mathrm{H}$ catalyst simulator

Controls the temperature of the $20.0 \mathrm{~K}$

to $29.1 \mathrm{~K}$ para- $\mathrm{H}$ catalyst simulator

Acquires measurement data

Controls the $\mathrm{H}_{2}$ flow rate

1

Controls movement of the magnets

1

Controls temperature of the 1st stage regenerator bed

Controls the temperature of the 2nd 


\subsection{Control Logic Specifications}

Specifications for the control logic include the following items:

- The implementation of the control and the data acquisition system shall be combined to simplify data distribution and storage while minimizing data latency.

- A modified hierarchical control strategy shall be incorporated to manage multiple control loop requirements with varying coupling of data, control, and goals.

- The control system shall be nearly autonomous.

- The safety control subsystem shall be entirely autonomous.

- A Real Time Graphical User Interface (RTGUI) to the control and data acquisition system shall be implemented.

- The RTGUI shall display real time user selections of data and/or control parameters.

- The RTGUI allows the user to gain limited real time access to modify the control system parameters and/or configuration.

- Acquired measurement data and control parameters shall be stored with time tags.

- The safety and other control loops shall be designed per applicable safety guidelines of OSHA.

\subsection{Operational Flexibility}

The control and data acquisition system shall be designed to include the following capabilities to maintain optimum operational flexibility:

- Variable user-defined frequencies for the control loops, including the magnet drive loop.

- Variable user-defined magnet position and velocity commands.

- Variable user-defined temperature set points for all temperature controllers.

- Real time user-defined caution signals in the safety control loop. Variable userdefined data sampling rates for measurement data.

- Variable user-defined data storage rates for measurement and control system data.

- Variable user-defined $\mathrm{H}_{2}$ and He flow rates.

\subsubsection{Anticipated Operating Parameters}

Liquefier testing will be aimed at mapping out a performance envelope over a range of operating parameters to find the optimum operating point. The control system flexibility provides a way to investigate this performance envelope. 
The primary baseline operating parameters around which testing will be conducted are delineated below.

- helium flow rate $436 \mathrm{~g} / \mathrm{s}$ (1st stage), $87 \mathrm{~g} / \mathrm{s}$ (2nd stage), $10 \mathrm{~g} / \mathrm{s}$ O/P Heat Exchanger (all intermittent flows, $0.5 \mathrm{~s}$ duration)

- hydrogen flow rate $1 \mathrm{~g} / \mathrm{s}$ continuous

- magnetic field $7 \mathrm{~T}$ (1st and 2nd stages)

- cycle time $2.0 \mathrm{~s}$ with $0.5 \mathrm{~s}$ dwell followed by $0.5 \mathrm{~s}$ blow.

Additional parameters such as magnet velocity, cryogen levels, vacuum levels, and so on are either not directly related to, or are of secondary importance relative to the primary parameters listed above.

\section{Selection of Magnetic Refrigerants for the Prototype Liquefier}

\subsection{Choice of Materials}

For the AMR cycle applied to the $20 \mathrm{~K}$ to $80 \mathrm{~K}$ temperature span, the heat capacity of the helium heat transfer fluid is nearly constant and small compared to the bed material. In this case, we have shown in the section on the magnetic working bed design that optimum refrigeration performance requires a magnetic regenerator bed with the property that the adiabatic temperature change with change in magnetic field, $\Delta \mathrm{T}_{\mathrm{s}}$, increases with increasing absolute temperature, $\mathrm{T}$. Each stage of the magnetic liquefier spans a relatively large range of temperature over which the $\Delta \mathrm{T}_{\mathrm{s}}$ of the magnetic material should have the correct temperature dependence, making this the primary criterion for the selection of the magnetic refrigerants.

The $\Delta \mathrm{T}_{\mathrm{s}}$, which measures the strength of the magnetocaloric effect, should also be relatively large to minimize adverse effects of the temperature difference between the bed and heat transfer fluid on the overall efficiency of the liquefier. This temperature difference is the factor $\left(t_{b}-t_{f}\right)$ appearing in equation 7 in the AMR model section. It is non-zero for a non-zero span $T_{h}-T_{c}$ of the refrigerator because the heat transfer coefficient $\mathrm{h}$ and heat capacity $\mathrm{c}_{\mathrm{p}}$ are fixed by the properties of helium, the bed area $\mathrm{A}$ is limited by the tolerable pressure drop, and the mass flow rate must be large enough to provide useful cooling power. If $\Delta \mathrm{T}_{\mathrm{s}}$ is not much larger than $\mathrm{t}_{\mathrm{b}}-\mathrm{t}_{\mathrm{f}}$, then one cannot achieve the zero-reduced period limit. Moreover, the approximations leading to equation 19 are no longer valid.

The heat capacity $C_{b}$ of the magnetic refrigerant should also be large. The cooling power per unit bed mass increases with increasing heat capacity of the material. This is because a larger heat capacity allows a larger flow rate of the heat transfer fluid for a given reduced period. If the reduced period becomes too large, the temperature of the gas leaving the bed at the cold end rises above $20 \mathrm{~K}$ and ceases to do useful cooling. 
Several other properties of the magnetic refrigerant are also important. First, the magnetization process should be thermodynamically reversible. Irreversibilities, observed as magnetic hysteresis, produce heating in the bed as the field is changed, and thus reduces both cooling power and efficiency. Magnetostriction, associated, as is hysteresis, with large magnetic anisotropy, is also an undesirable property. Finally, thermal conductivity of the bed material must be large enough to allow extraction of the heat of magnetization without forming a substantial temperature gradient within the particles. The small size of the bed particles, a requirement for efficient heat transfer, makes this last requirement easy to meet. ${ }^{17}$

We have chosen to use one material per stage of the tenth-scale liquefier to simplify fabrication and ensure rapid cooldown. The $\Delta \mathrm{T}_{\mathrm{s}}$ increasing with temperature is obtained, in this case, by choosing a ferromagnetic material with a sharp Curie point (the magnetic ordering temperature) equal to the maximum operating temperature of the stage. In the region of the Curie point, both $\Delta \mathrm{T}_{\mathrm{s}}$ and $\mathrm{C}_{\mathrm{b}}$ attain maxima. A large value of the total atomic angular momentum $J$ will increase the values of these maxima because the total magnetic entropy is proportional to $\ln (2 \mathrm{~J}+1)$. Magnetic hysteresis and magnetostriction are potential problems if the material is operated well below its Curie point in cases where magnetic atoms in the structure have orbital angular momentum. Additional considerations are that the material should be neither excessively expensive nor extremely toxic.

The selection of the magnetic refrigerants was done using the above criteria. The non-radioactive magnetic elements are the transition metals $\mathrm{Mn}, \mathrm{Fe}, \mathrm{Co}, \mathrm{Ni}$; the light rare earths $\mathrm{Ce}, \mathrm{Pr}, \mathrm{Nd}$, and $\mathrm{Sm}$; and the heavy rare earths $\mathrm{Eu}, \mathrm{Gd}, \mathrm{Tb}, \mathrm{Dy}, \mathrm{Ho}, \mathrm{Er}, \mathrm{Tm}$, and $\mathrm{Yb}$. The transition metals have small values of $\mathrm{J}$ and too high ordering temperatures in compounds with a high concentration of magnetic atoms. The high ordering temperatures arise because there is a large overlap between the 3D electron orbitals responsible for the magnetism in the transition metals. The overlap produces high ordering temperatures and quenches the orbital angular momentum, reducing J. The light rare earths (see Table 10) have relatively small g-factors, thereby reducing the strength of the interaction $\mathrm{g \mu H}$ with the magnetic field; crystal field splitting in these materials is also very strong, reducing the amount of magnetic entropy available. The heavy rare earths $\mathrm{Eu}, \mathrm{Tb}$, and $\mathrm{Tm}$ are too rare and expensive for large scale use. Crystal field splittings and magnetostriction tend to be very large for Dy compounds, and the $\mathrm{g}$ and $\mathrm{J}$ values of $\mathrm{Yb}$ are too small. This leaves compounds of $\mathrm{Gd}, \mathrm{Ho}$, and $\mathrm{Er}$ for consideration. Gd has no orbital angular momentum and is the cheapest of the three, so it is the preferred magnetic element. Unfortunately, Gd has the largest DeGennes factor, $(g-1)^{2} \mathrm{~J}(\mathrm{~J}+1)$, of the rare earths (see Table 10 ). This implies that magnetic ordering temperatures, which are approximately proportional to the DeGennes factor, are relatively high. Hence there are few gadolinium compounds with a high concentration of the magnetic species that order below $50 \mathrm{~K}$. Hence, erbium compounds have also been considered for the lower temperature stage of the liquefier. 
Table 10. The Magnetic Elements

\begin{tabular}{|l|l|l|l|}
\hline Magnetic Element & $\mathrm{J}$ & $\mathrm{g}$ & $(\mathrm{g}-1)^{2} \mathrm{~J}(\mathrm{~J}+1)$ \\
\hline $\mathrm{Mn}$ & $5 / 2$ & 2 & $(8.7500)$ \\
\hline $\mathrm{Fe}$ & 2 & 2 & $(6.0000)$ \\
\hline $\mathrm{Co}$ & $3 / 2$ & 2 & $(3.7500)$ \\
\hline $\mathrm{Ni}$ & 1 & 2 & $(2.0000)$ \\
\hline $\mathrm{Ce}$ & $5 / 2$ & $6 / 7$ & 0.1786 \\
\hline $\mathrm{Pr}$ & 4 & $4 / 5$ & 0.8000 \\
\hline $\mathrm{Nd}$ & $9 / 2$ & $8 / 11$ & 1.8409 \\
\hline $\mathrm{Sm}$ & $5 / 2$ & $2 / 7$ & 4.4643 \\
\hline $\mathrm{Eu}$ & $7 / 2$ & 2 & 15.7500 \\
\hline $\mathrm{Gd}$ & $7 / 2$ & 2 & 15.7500 \\
\hline $\mathrm{Tb}$ & 6 & $3 / 2$ & 10.5000 \\
\hline $\mathrm{Dy}$ & $15 / 2$ & $4 / 3$ & 7.0833 \\
\hline $\mathrm{Ho}$ & 8 & $5 / 4$ & 4.5000 \\
\hline $\mathrm{Er}$ & $15 / 2$ & $6 / 5$ & 2.5500 \\
\hline $\mathrm{Tm}$ & 6 & $7 / 6$ & 1.1667 \\
\hline $\mathrm{Yb}$ & $7 / 2$ & $8 / 7$ & 0.3214 \\
\hline
\end{tabular}

Note: The magnetic elements, together with their spin $\mathrm{J}$ and gyromagnetic ratio $\mathrm{g}$. Large values of $\mathrm{J}$ and $\mathrm{g}$ are desirable for magnetic refrigerants. The Curie temperatures of compounds of the rare earth elements (Ce through $\mathrm{Yb}$ ) are proportional to the deGennes factor, $(\mathrm{g}-1)^{2} \mathrm{~J}(\mathrm{~J}+1)$.

The pure rare earths do not have suitable Curie points in the $20 \mathrm{~K}$ to $80 \mathrm{~K}$ range ${ }^{19}$, but many of their compounds do. Compounds of $\mathrm{Gd}$ were the prime candidates for the magnetic refrigerants for the reasons noted above. We restricted ourselves to intermetallic compounds because the non-metallic compounds generally have low thermal conductivities and ordering temperatures below $20 \mathrm{~K}$. Alloys between two rare earths did not have suitable properties, with the exception of some erbium alloys to be discussed. Non-radioactive metallic or semiconducting compounds with $\mathrm{Gd}$ are formed by the elements $\mathrm{Be}, \mathrm{Al}, \mathrm{Si}, \mathrm{P}, \mathrm{Mn}, \mathrm{Fe}, \mathrm{Co}, \mathrm{Ni}, \mathrm{Cu}, \mathrm{Zn}, \mathrm{Ga}, \mathrm{Ge}, \mathrm{As}, \mathrm{Se}$, $\mathrm{Ru}, \mathrm{Rh}, \mathrm{Pd}, \mathrm{Ag}, \mathrm{Cd}$, In, Sn, Sb, Te, Re, Os, Ir, $\mathrm{Pt}, \mathrm{Au}, \mathrm{Hg}, \mathrm{Tl}, \mathrm{Pb}$, and $\mathrm{Bi}$. $\mathrm{Rh}, \mathrm{Re}$, $\mathrm{Os}$, Ir, and $\mathrm{Pt}$ were rejected as too expensive. $\mathrm{Be}, \mathrm{As}, \mathrm{Hg}$, and $\mathrm{Tl}$ are too toxic. $\mathrm{P}$, $\mathrm{Zn}, \mathrm{Se}, \mathrm{Cd}, \mathrm{Sb}, \mathrm{Te}, \mathrm{Pb}$ and $\mathrm{Bi}$ have high vapor pressure at the melting temperature of $\mathrm{Gd}$ and the compounds are thus hard to form with good stoichiometry. All compounds with $\mathrm{Fe}, \mathrm{Ag}$, and $\mathrm{Au}$ and most with $\mathrm{Mn}, \mathrm{Co}$, and $\mathrm{Cu}$ are ferrimagnetic or 
with $\mathrm{Fe}, \mathrm{Ag}$, and $\mathrm{Au}$ and most with $\mathrm{Mn}, \mathrm{Co}$, and $\mathrm{Cu}$ are ferrimagnetic or antiferromagnetic and hence not suitable. Most compounds with $\mathrm{Si}, \mathrm{Ga}$, and $\mathrm{Ge}$ are antiferromagnetic; those that are ferromagnetic have strong anisotropies that make them unsuitable. The remaining possibilities are $\mathrm{Al}, \mathrm{Ni}, \mathrm{Pd}, \mathrm{Ru}, \mathrm{In}$, and $\mathrm{Sn}$. Among these we have found that the ferromagnetic compounds $\mathrm{GdAl}_{2}, \mathrm{ErAl}_{2}, \mathrm{GdNi}, \mathrm{GdNi}_{2}$, and $\mathrm{GdPd}$ have interesting properties. Ternaries formed by partly substituting other elements such as $\mathrm{Co}$ or $\mathrm{Cu}$ for $\mathrm{Ni}$ in $\mathrm{GdNi}_{2}$ and $\mathrm{GdNi}$ also show some promise. Finally, among the rare earth metals, erbium has a $19 \mathrm{~K}$ ferromagnetic transition $\mathrm{T}_{c}$ whose magnetocaloric potential is unfortunately suppressed by an $80 \mathrm{~K}$ antiferromagnetic transition $T_{N}$, but addition of the non-magnetic rare earth lanthanum raises $T_{c}$ and lowers $T_{N^{*}}$. If sufficient $\mathrm{La}$ is added, $\mathrm{T}_{\mathrm{c}}$ exceeds $\mathrm{T}_{\mathrm{N}}$, allowing a substantial magnetocaloric effect.

Using the above considerations, we chose to measure the magnetocaloric properties of the binary compounds $\mathrm{GdPd}, \mathrm{GdNi}$, and $\mathrm{GdNi}_{2}$, the alloy $\mathrm{Er}_{0.8} \mathrm{La}_{0.2}$, and the ternaries $\mathrm{GdPd}_{0.75} \mathrm{Ni}_{0.25}, \mathrm{GdNi}_{0.95} \mathrm{Co}_{0.05}$, and $\mathrm{GdNi}_{0.22} \mathrm{Cu}_{0.78}$. We also report on measurements done prior to the start of this contract on $\mathrm{Er}_{0.86} \mathrm{Gd}_{0.14} \mathrm{Al}_{2}$ and $\mathrm{Er}_{0.75} \mathrm{Dy}_{0.25} \mathrm{Ni}$. The specific properties measured were the heat capacity at constant magnetic field, $C_{B}$, and the adiabatic temperature change upon change in field $\Delta T_{s}$; these properties characterize the behavior of the magnetic material in the isofield and adiabatic legs of the AMR cycle. In addition, we have measured the magnetization, $\mathrm{M}$, of the materials. The magnetization is required to calculate the magnetic forces between the material bed and the magnet, and also serves as a rapid screening technique for potential magnetic refrigerants.

\subsection{Magnetization Measurement Instrumentation}

A magnetometer measures the magnetic moment, $M$, of a sample, typically as a function of magnetic field and temperature. A magnetometer combined with a variable temperature cryostat, temperature control system, and data acquisition system becomes a powerful tool for investigating the magnetic properties of ferromagnetic refrigerants.

During Phasé I, we integrated an existing manual Vibrating Sample Magnetometer (PAR model 155) with an existing Cryo Industries variable temperature cryostat and purchased an $8 \mathrm{~T}$ superconducting magnet and power supply. An IBM-compatible personal computer was obtained for control and data acquisition. The thermal system response was characterized and a control system was developed for implementation on the PC.

The magnetometer is of value to this program in three ways. First, it provides a rapid tool for screening magnetic materials. In contrast to heat capacity measurements, which involve several days of sample preparation and a week or more of data collection per specimen, the magnetometer can in less than a day determine a specimen's magnetic ordering temperature and geometry, as well as give some indication of the 
sharpness of the transition and amount of magnetic entropy available. Thus only the best materials need be characterized further via heat capacity and $\Delta \mathrm{T}_{\mathrm{s}}$ measurement (which is still the definitive characterization for the AMR). The second use for magnetization data is for calculating forces acting between the magnetic beds and magnets. The force is directly proportional to the magnetization of the bed material. The third use is for measuring magnetic hysteresis. The area contained inside the closed $\mathrm{M}(\mathrm{H})$ curve is proportional to the hysteretic work, $\mathrm{W}_{\mathrm{h}}$, dissipated in magnetizing and demagnetizing the material. These measurements require excellent control of magnetic field and temperature. $\mathrm{W}_{\mathrm{h}}$ can also be obtained from $\Delta \mathrm{T}_{\mathrm{s}}$ measurements, but such measurements are cumbersome for any field change cycle not consisting of the simple application of and complete removal of the field.

Samples for magnetization measurement were made in the form of thin chips aligned with their long axis parallel to the field to minimize demagnetization effects. The high sensitivity of the magnetometer allowed use of much smaller samples than those used for heat capacity measurements. Hence multiple samples could be cut from one arc-melt specimen to check specimen homogeneity. The samples were fixed to a non-magnetic sample holder with grease that solidifies at low temperature to make a rigid mount.

\subsubsection{Magnetization Measurements}

Magnetization measurements were made on the pseudo-binary series $\operatorname{GdPd}_{1-\mathrm{x}} \mathrm{Ni}_{\mathrm{x}}$ and $\mathrm{Gd}\left(\mathrm{Cu}_{1-\mathrm{x}} \mathrm{Ni}_{\mathrm{x}}\right)_{2}$ to determine the dependence of the Curie point and the saturation magnetization upon composition. Figure 77 shows the effect of varying the composition variable $\mathrm{x}$ in $\mathrm{GdPd}_{1-\mathrm{x}} \mathrm{Ni}_{\mathrm{x}}$. Note that the Curie point, marked by the region of greatest $\mathrm{dM} / \mathrm{dT}$, changes smoothly between $38 \mathrm{~K}$ and $70 \mathrm{~K}$. Moreover, the magnetization is monotonic in temperature and a substantial saturation magnetization, marked by the low temperature maximum in $\mathrm{M}$, is obtained. This verifies that the simple ferromagnetic ordering geometry does not change in this series. In contrast, the series $\mathrm{Gd}\left(\mathrm{Cu}_{1-\mathrm{x}} \mathrm{Ni}_{\mathrm{x}}\right)_{2}$ shows a complex temperature dependence of the magnetization and hence a mixed antiferromagnetic and ferromagnetic behavior (Figure 78).

Figure 79 shows the magnetization of $\mathrm{Er}_{0.8} \mathrm{La}_{0.2}$ as a function of field and temperature. This complete map of $\mathrm{M}(\mathrm{T}, \mathrm{B})$ permits calculation of the magnetic forces in the liquefier.

The magnetometer can also be used to directly measure magnetic hysteresis. Figure 79 shows the hysteresis loop $\mathrm{M}(\mathrm{H})$ for $\mathrm{GdCu}_{1.8} \mathrm{Ni}_{0.2}$. Note that although $0.3 \mathrm{~T}$ is required to drive the material out of the antiferromagnetic state (marked by the region of small $\mathrm{dM} / \mathrm{dH}$ near the origin), the hysteresis is much smaller, approximately $0.06 \mathrm{~T}$. 


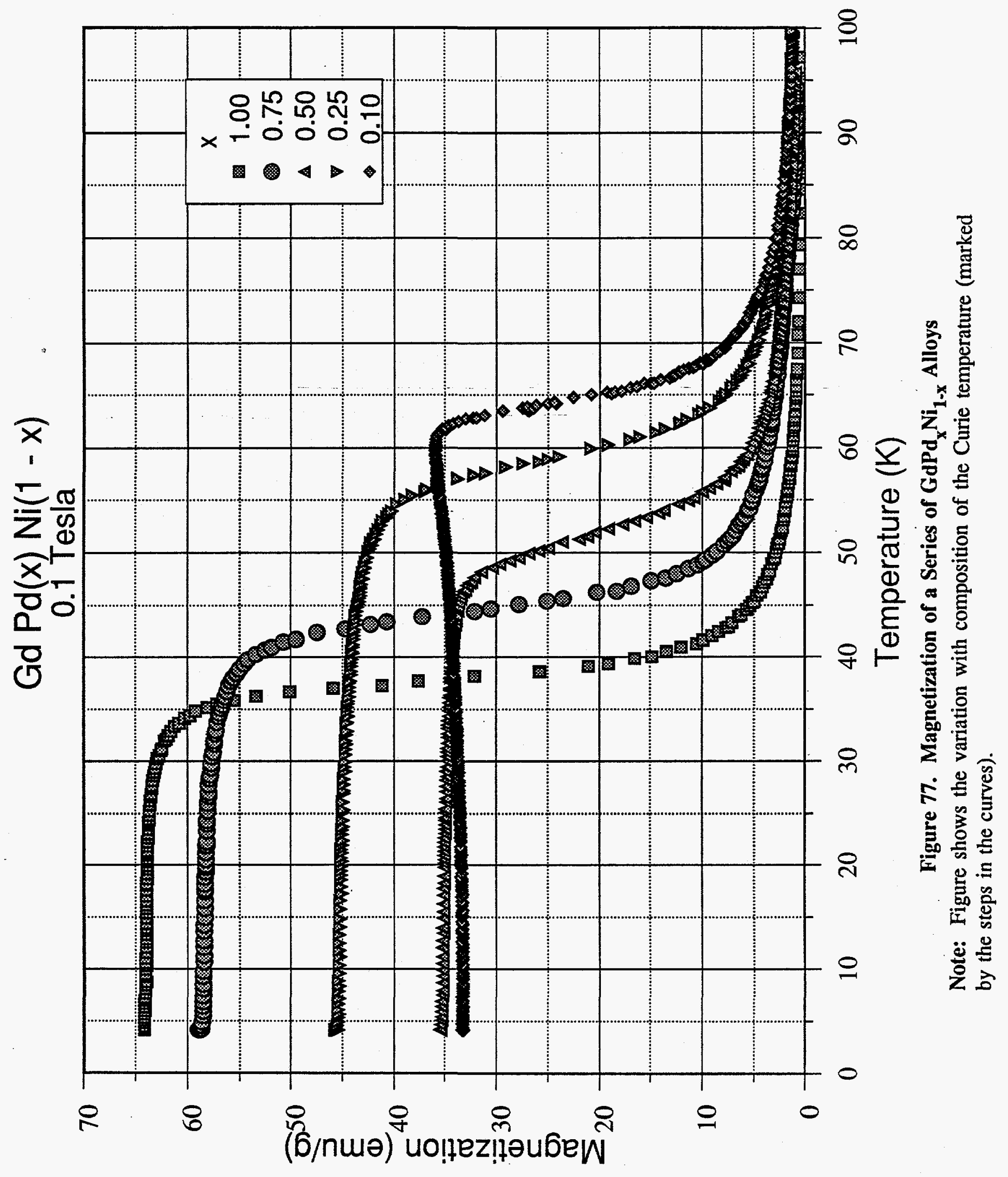




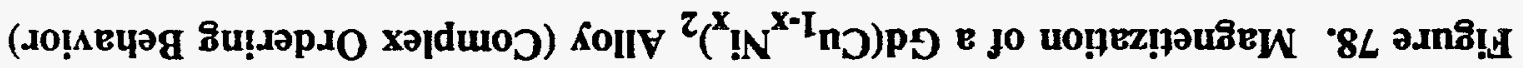

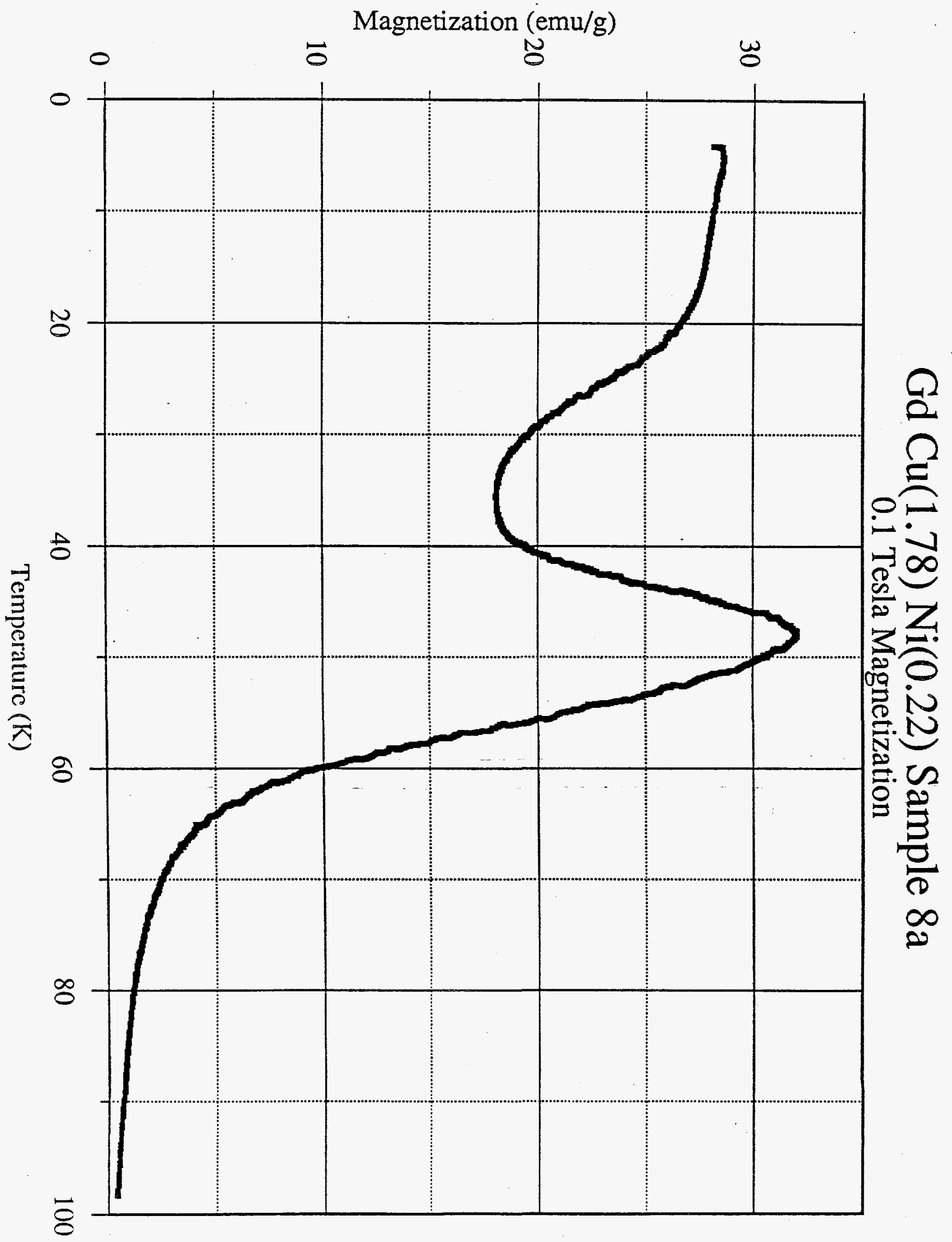




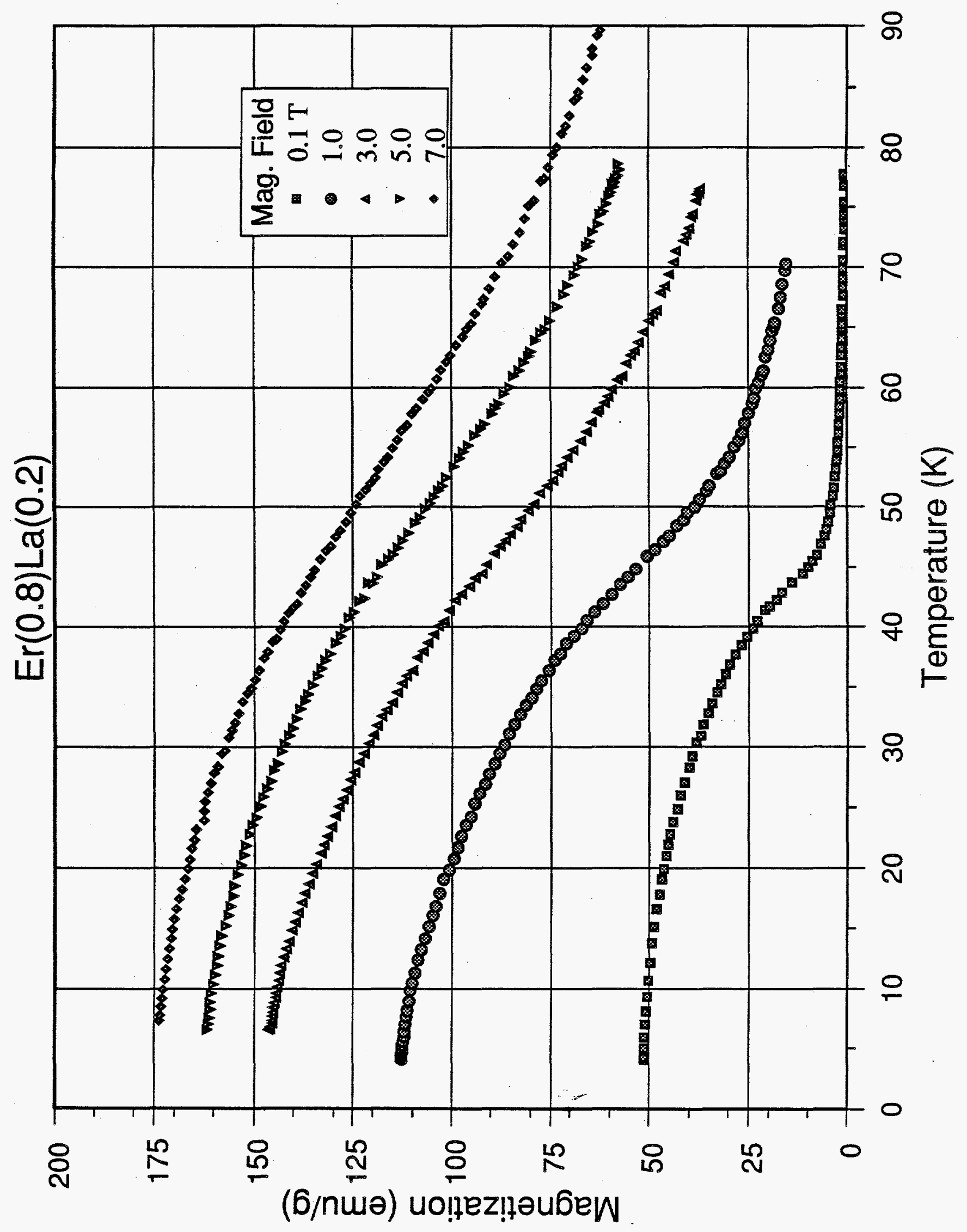

Figure 79. Magnetization of $\mathrm{Er}_{0.8} \mathrm{La}_{0.2}$ for Various Applied Magnetic Fields 


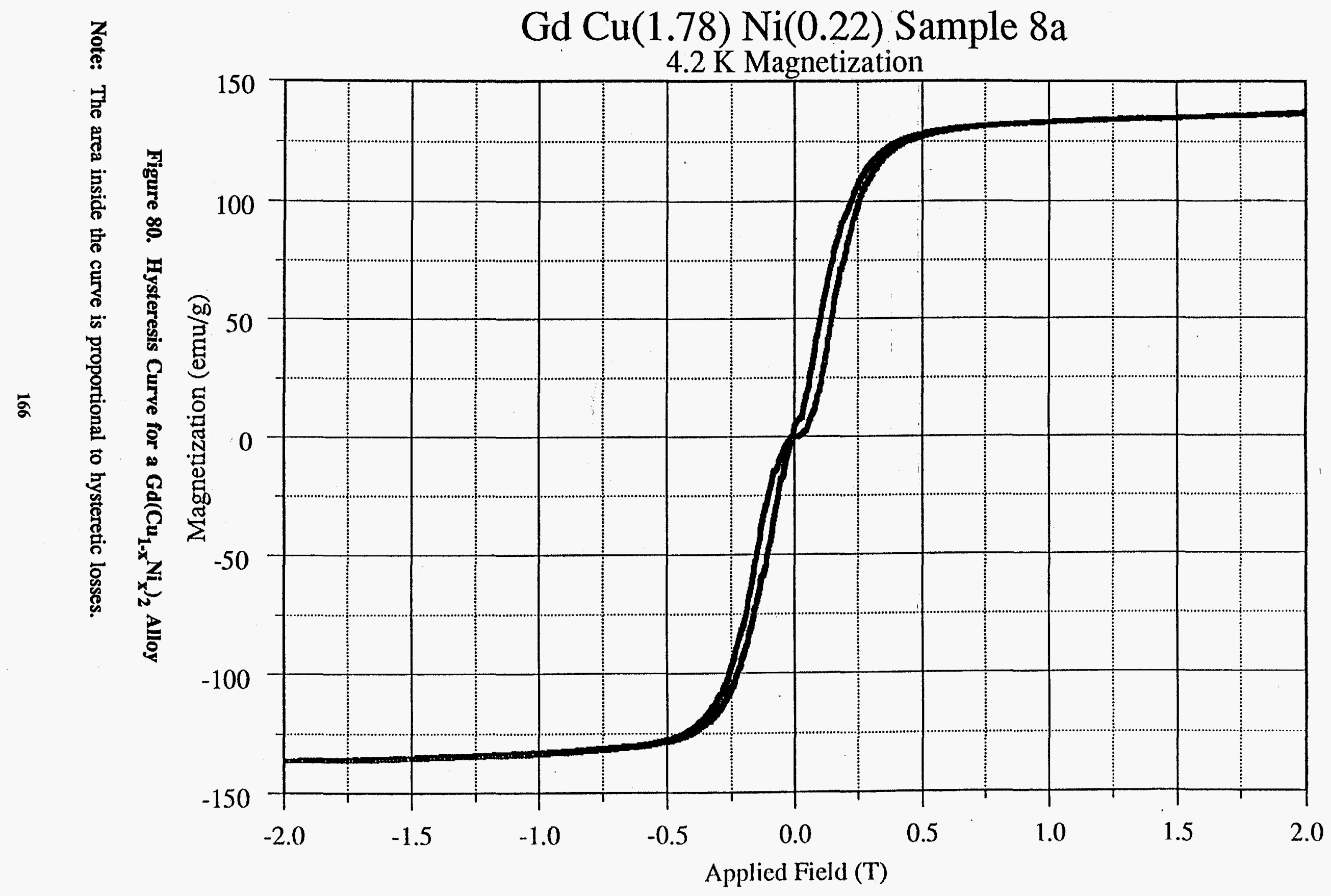




\subsection{Magnetocaloric Effect Measurement Techniques}

$\mathrm{GdNi}$ and $\mathrm{GdPd}$ are congruently melting compounds with the orthorhombic $\mathrm{CrB}$ structure. Our specimens were prepared by arc-melting the component elements in an argon atmosphere over a water-cooled copper hearth. Phase purity was verified by metallography. $\mathrm{GdNi}_{2}$ is a Laves phase compound with the cubic $\mathrm{MgCu}_{2}$ structure. Although $\mathrm{GdNi}_{2}$ is reported to be incongruently melting ${ }^{19}$, we obtained specimens of satisfactory phase purity by arc-melting. Attempts to form a specimen by sintering were not successful. Ternary compounds with two transition metals, such as $\mathrm{GdPd}_{0.75} \mathrm{Ni}_{0.25}$ were normally formed by first reacting the two transition metals in the arc melter to form a disordered alloy in which diffusion was expected to be faster than in the intermetallic compound. The compound was then formed by reacting the transition metal alloy with the rare earth. $\mathrm{ErAl}_{2}$ and $\mathrm{GdAl}_{2}$ are congruently-melting $\mathrm{MgCu}_{2}$ Laves phase compounds that exhibit solid-solid solubility. Arc-melted specimens of $\mathrm{Er}_{0.86} \mathrm{Gd}_{0.14} \mathrm{Al}_{2}$ were prepared by first melting $\mathrm{Gd}$ and $\mathrm{Er}$ together, then reacting with Al. These specimens were prone to cracking during solidification. Standard powder metallurgy techniques and pressureless sintering were used to obtain a specimen for measurements. $\mathrm{Er}_{0.8} \mathrm{La}_{0.2}$ was made by arc melting the elements. Precautions were taken to minimize exposure of the pure $\mathrm{La}$ to air; the reacted alloy, however, was resistant to corrosion and could be handled normally. The specimen of $\mathrm{Er}_{0.75} \mathrm{Dy}_{0.25} \mathrm{Ni}$ was made by Ames Laboratory at Ames, Iowa; all other specimens were made inhouse.

The $\mathrm{C}_{\mathrm{B}}$ and $\Delta \mathrm{T}_{\mathrm{s}}$ measurements were done on 10 to $20 \mathrm{~g}$ samples suspended on fine linen threads in vacuum. The sample was surrounded by two isothermal cans maintained at the same mean temperature as the sample to minimize radiation and conduction losses. The magnetic field was provided by a superconducting solenoid immersed in liquid helium outside the sample chamber. The specimens with aspect ratios of about 5 to 1 were aligned with the magnetic field to minimize demagnetization effects, for which corrections were not made. The measurements were done by applying a known heat pulse or magnetic field change to the sample and observing the resulting temperature change. Corrections were made for heat losses to the surroundings and for thermal addenda. Corrections for the magnetoresistance of the carbonglass thermometer used were negligible. The experiment was automated except for startup and helium transfer. More details on the apparatus and method for doing the measurements have been reported previously. ${ }^{20}$

\subsubsection{Magnetocaloric Effect Measurement Results}

Figures 81 and 82 show the measured $C_{B}$ and $\Delta T_{s}$ of GdPd. Figures 83 and 84 show the corresponding measurements on GdNi. Limited heat capacity data on these compounds from a previous series of measurements has been reported. ${ }^{21,22}$ Figures 85 and 86 show $\mathrm{C}_{\mathrm{b}}$ and $\Delta \mathrm{T}_{\mathrm{s}}$ for $\mathrm{GdPd}_{0.75} \mathrm{Ni}_{0.25}$. Figures 87 and 88 show $\mathrm{C}_{\mathrm{b}}$ and $\Delta \mathrm{T}_{\mathrm{s}}$ for $\mathrm{GdNi}_{0.95} \mathrm{Co}_{0.05}$. Figures 89 and 90 show $\mathrm{C}_{\mathrm{B}}$ and $\Delta \mathrm{T}_{\mathrm{s}}$ for $\mathrm{GdNi}_{2}$. 


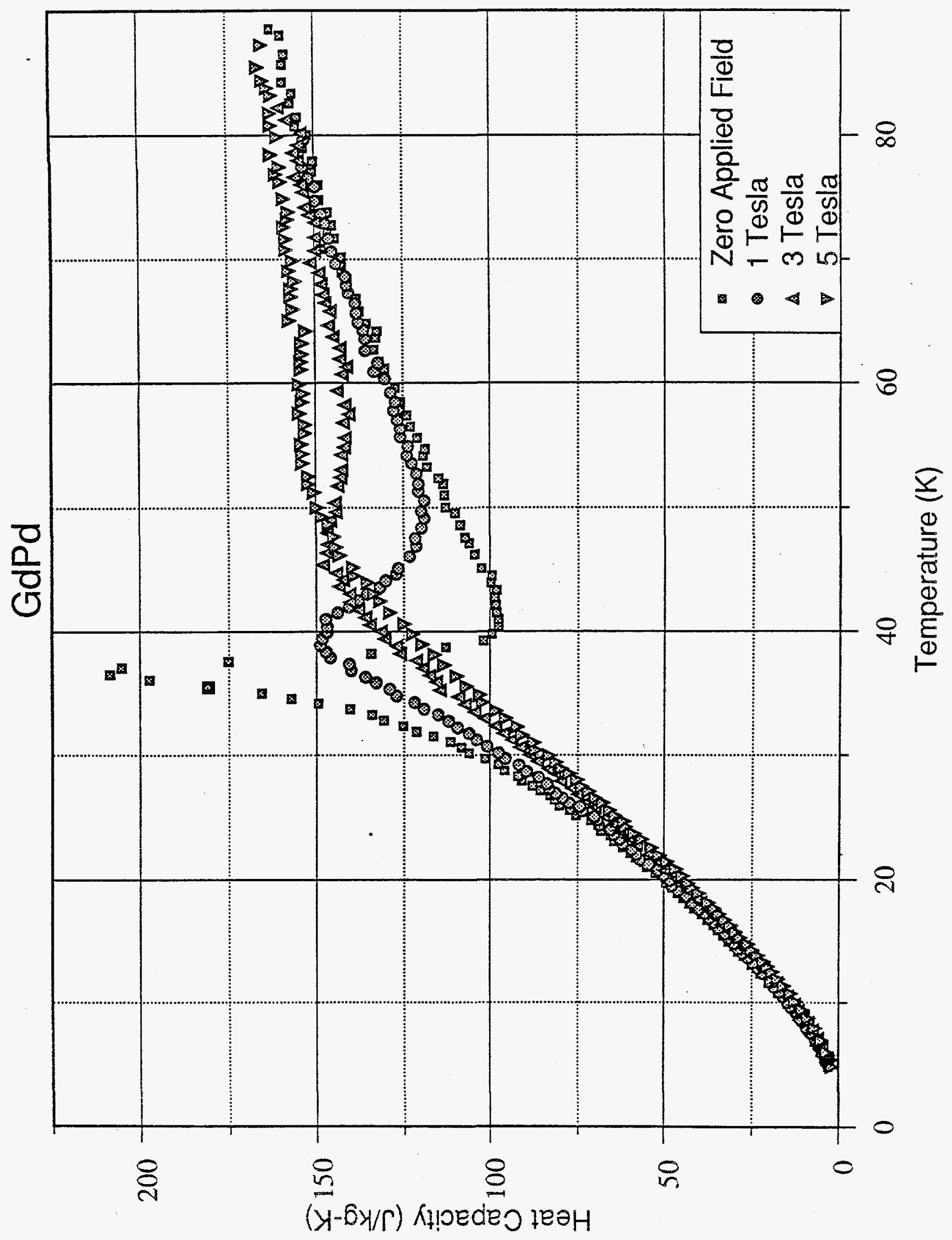

Figure 81. Heat Capacity, $\mathrm{C}_{\mathrm{B}}$, for $\mathrm{GdPd}$, for Various Applied Fields

Note: The sharp Curie point at $38 \mathrm{~K}$. 


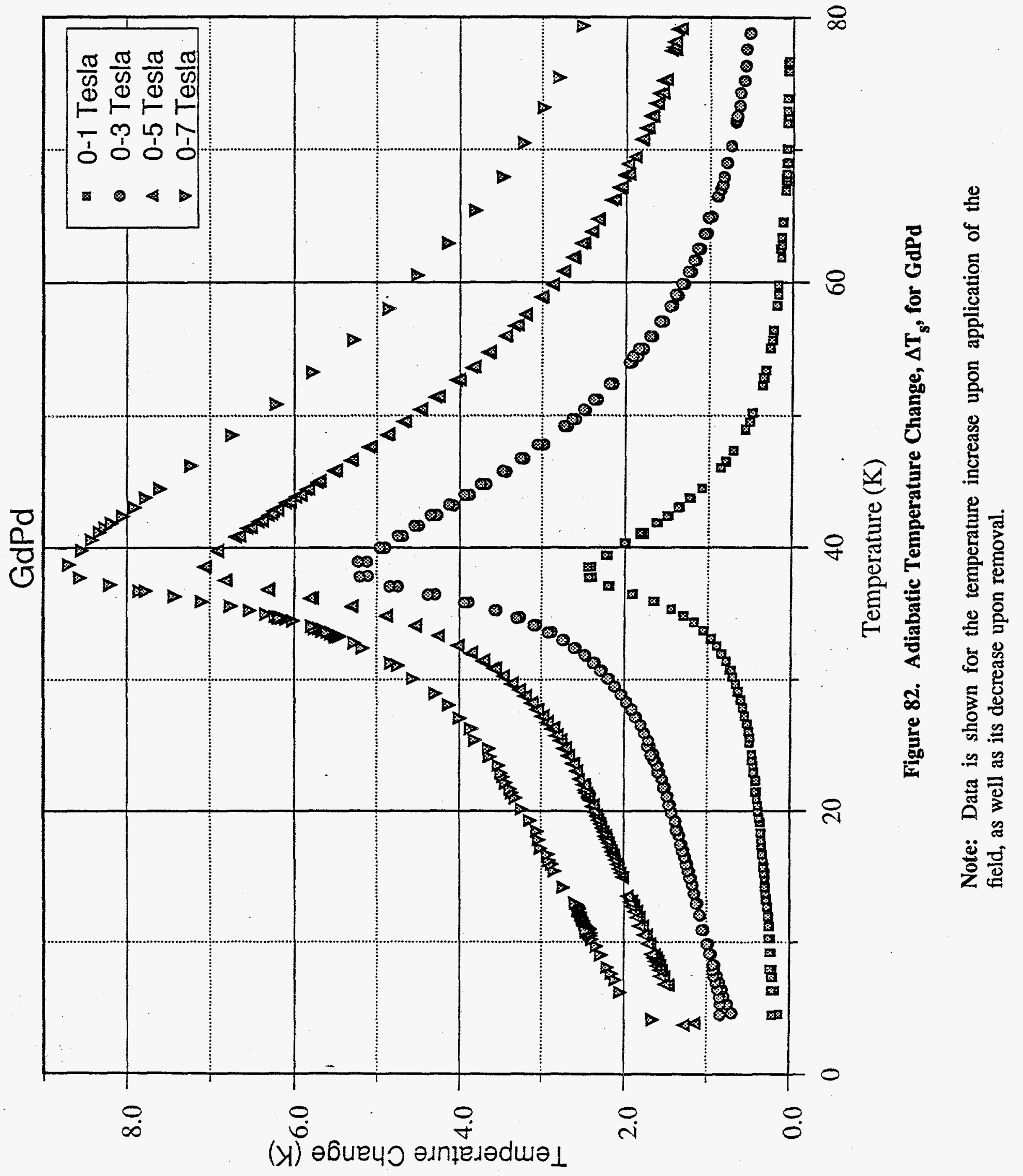




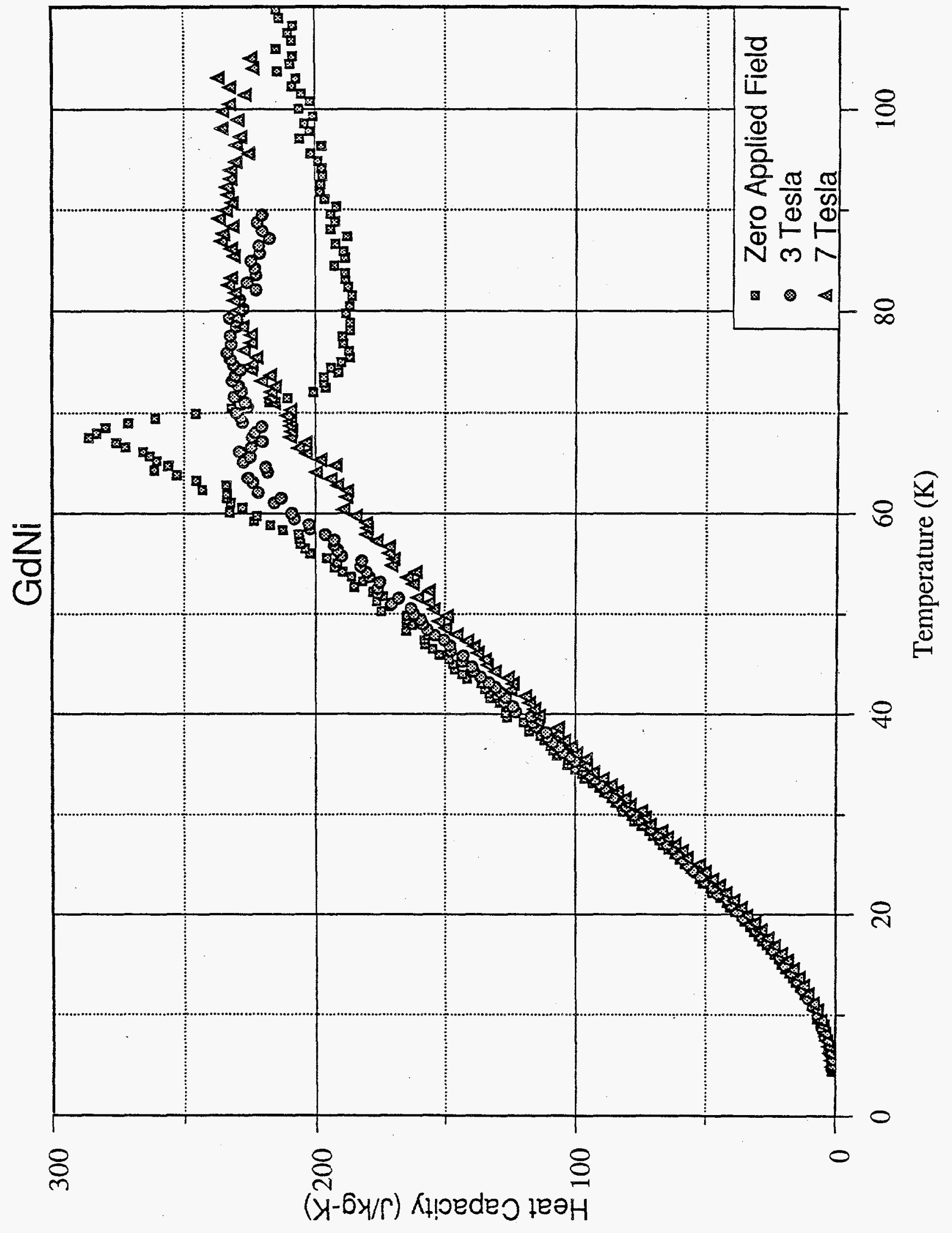

Figure 83. $C_{B}$ for $\mathrm{GdNi}$ 


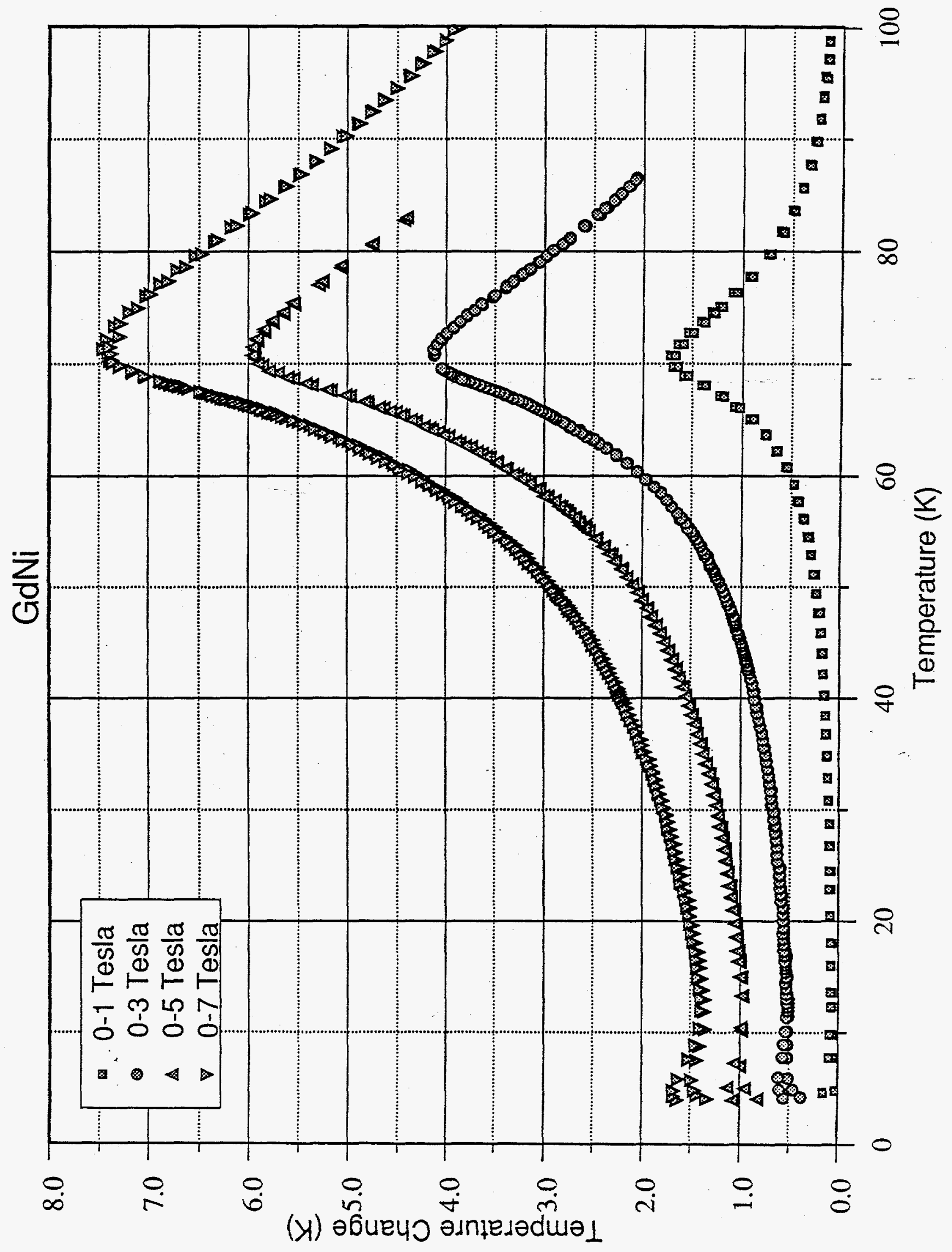

Figure 84. $\Delta \mathrm{T}_{\mathrm{s}}$ for $\mathrm{GdNi}$ 


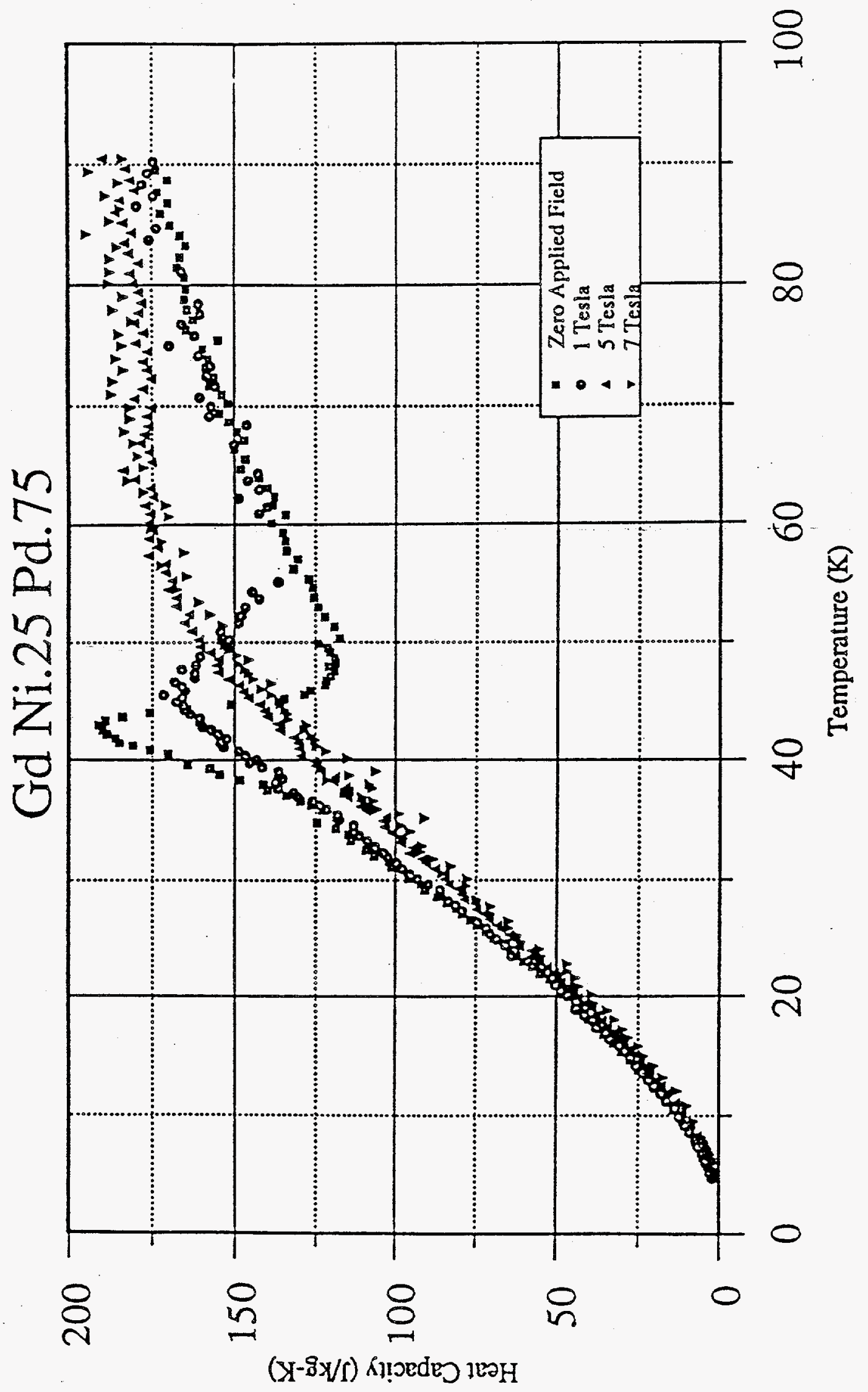

Figure 85. $\mathrm{C}_{\mathrm{B}}$ for $\mathrm{GdNi}_{0.25} \mathrm{Pd}_{0.75}$ 


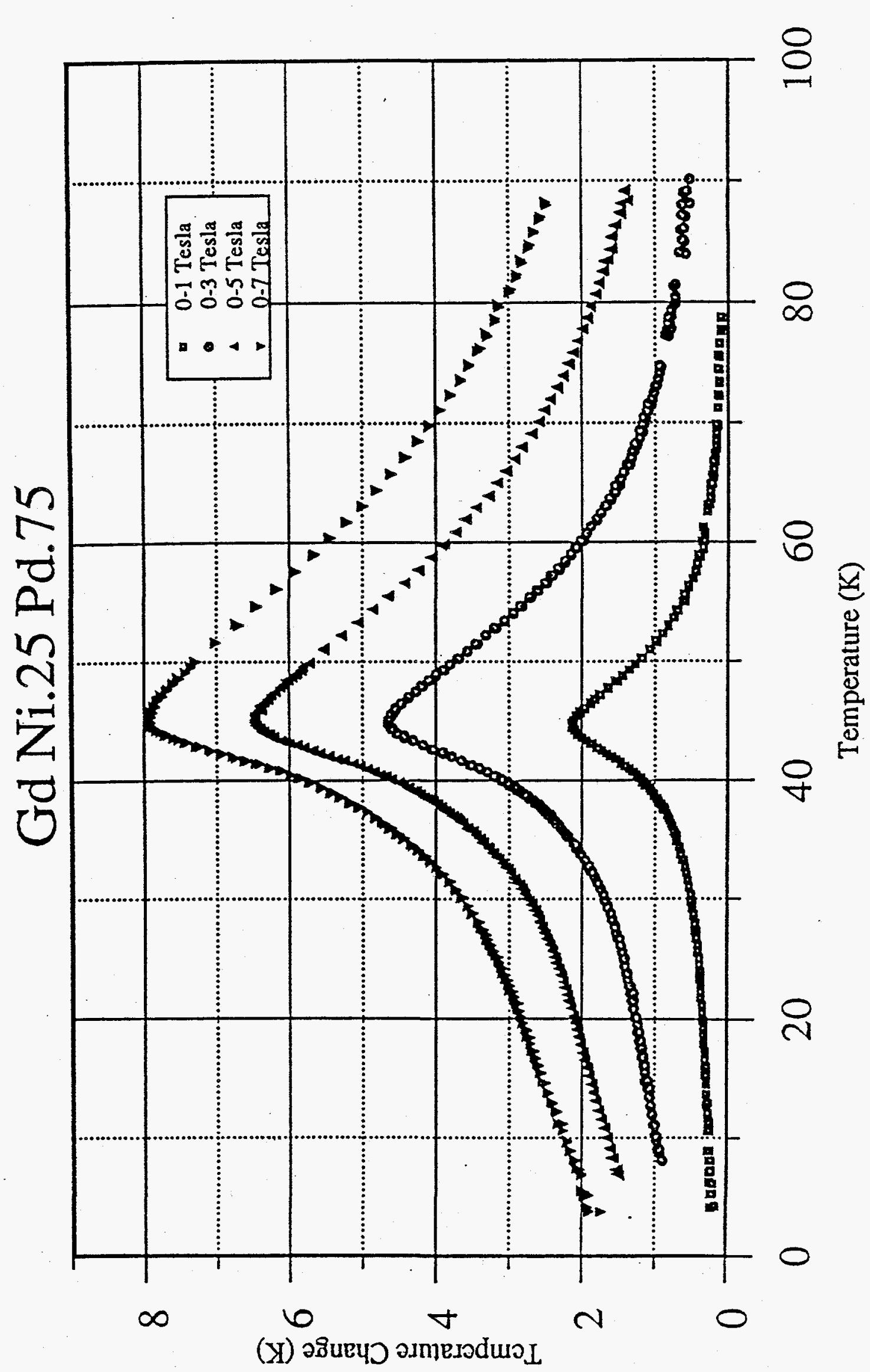

Figure 86. $\Delta \mathrm{T}_{\mathrm{s}}$ for $\mathrm{GdNi}_{0.25} \mathrm{Pd}_{0.75}$ 


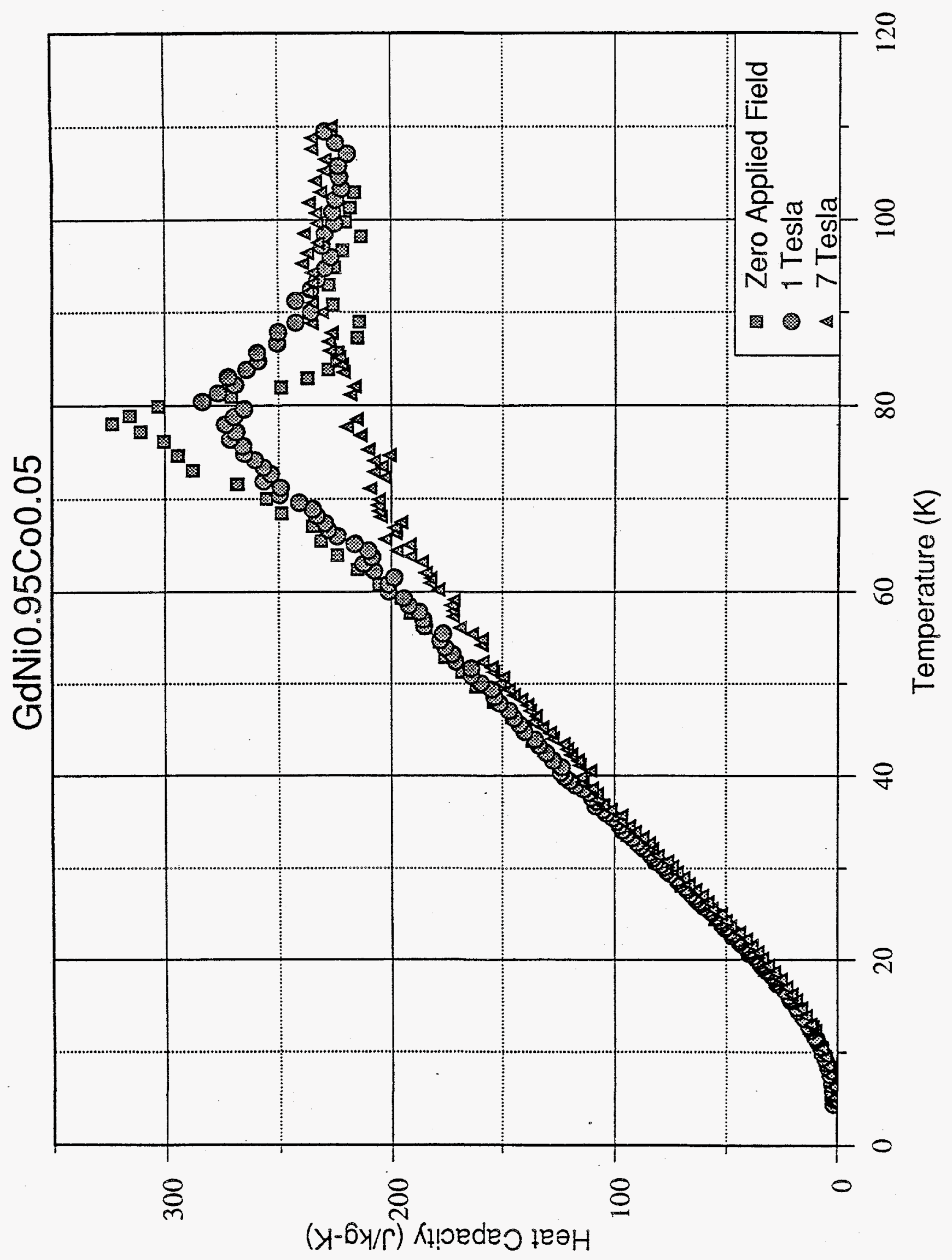

Figure 87. $\mathrm{C}_{\mathrm{B}}$ for $\mathrm{GdNi}_{0.95} \mathrm{Co}_{0.05}$ 
GdNi0.95Co0.05

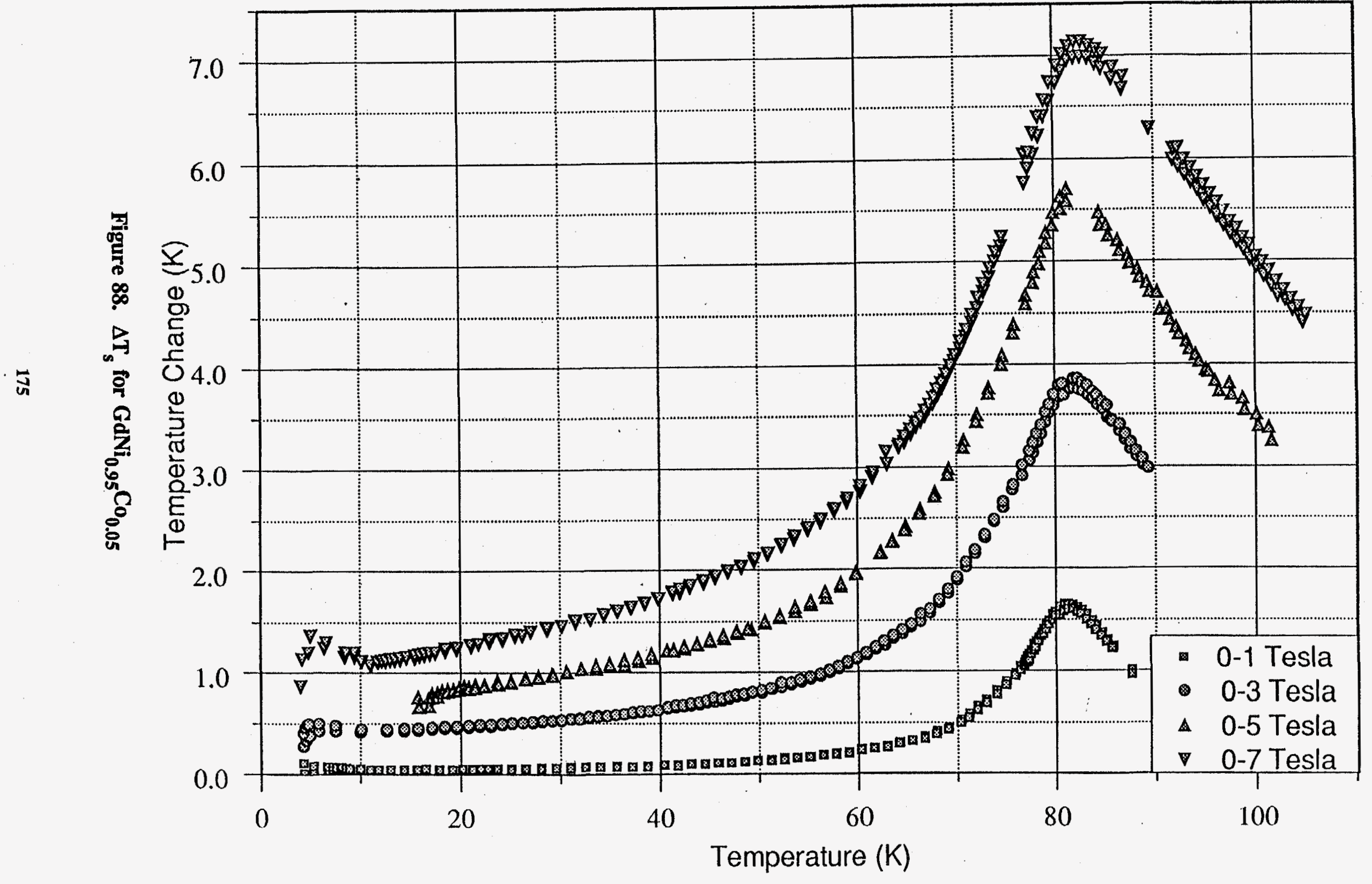




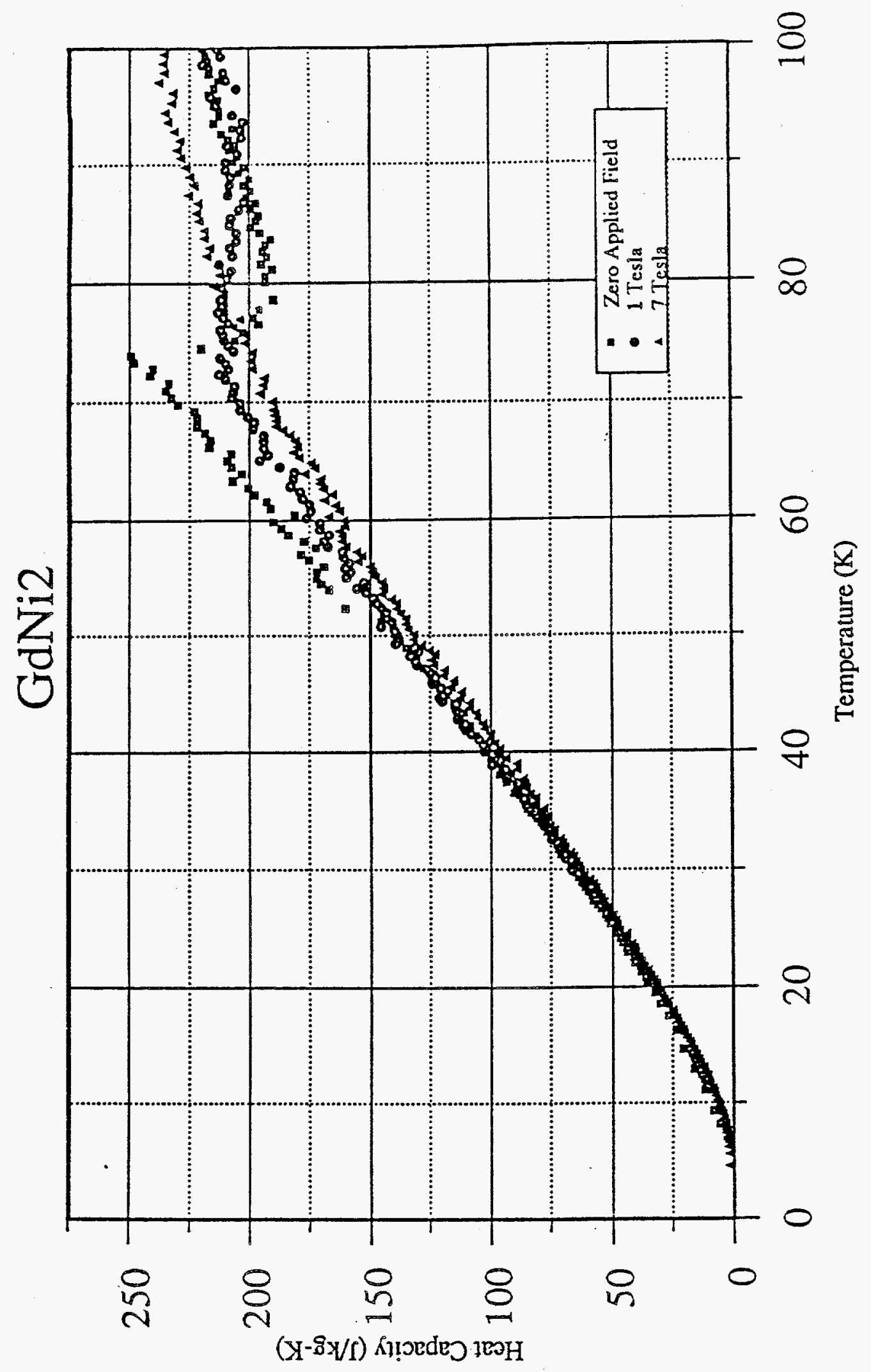

Figure 89. $\mathrm{C}_{\mathrm{B}}$ for $\mathrm{GdNi}_{2}$ 


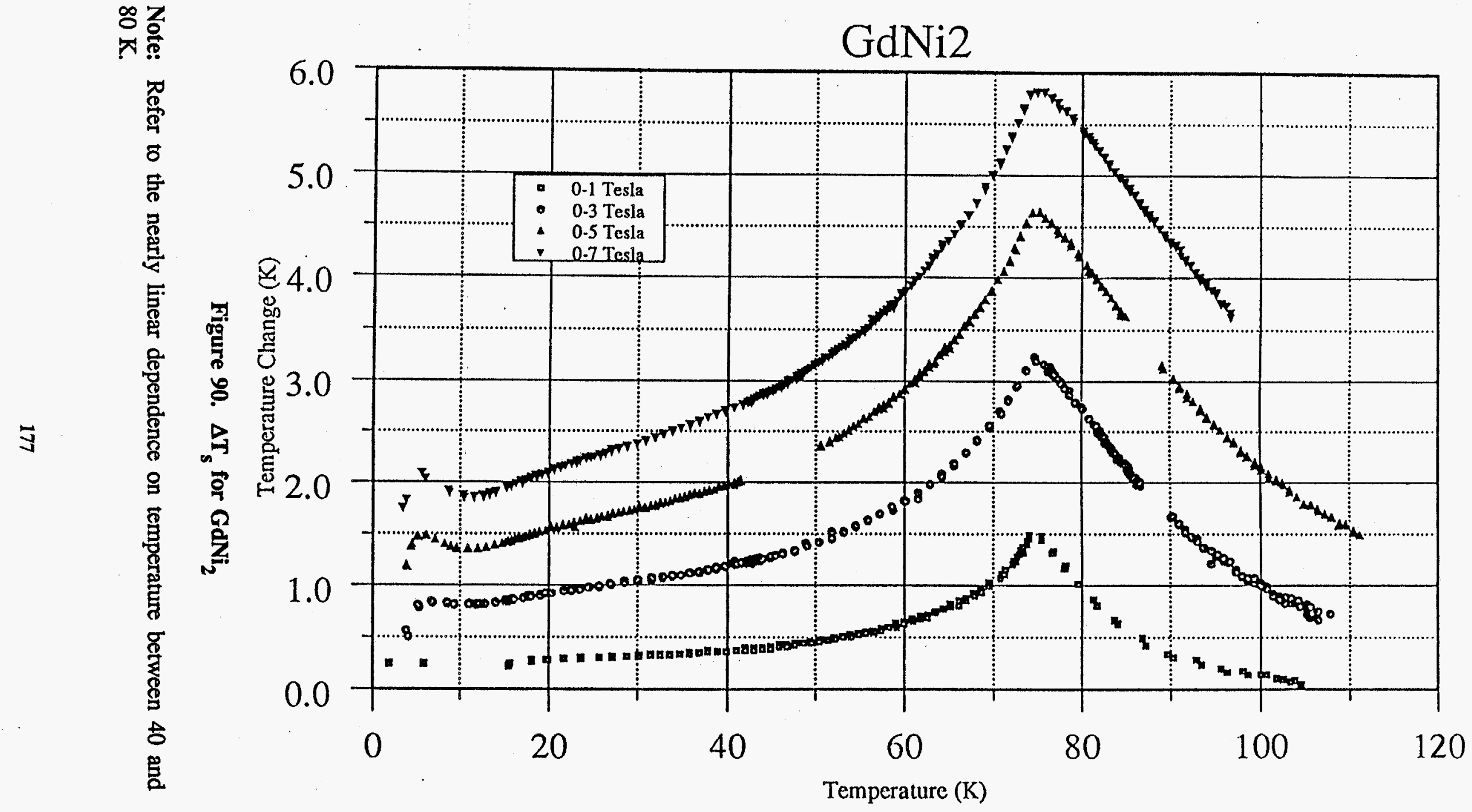


The data for GdPd, GdNi, GdPd ${ }_{0.75} \mathrm{Ni}_{0.25}, \mathrm{GdNi}_{0.95} \mathrm{Co}_{0.05}$, and $\mathrm{GdNi}_{2}$ each show a sharp transition, at $38 \mathrm{~K}, 70 \mathrm{~K}, 45 \mathrm{~K}, 81 \mathrm{~K}$, and $75 \mathrm{~K}$, respectively. These are marked by sharp lambda-type peaks in the zero field heat capacity and by peaks in the $\Delta \mathrm{T}_{\mathrm{s}}$ curves. The ferromagnetic character of the transition is verified by the action of applied magnetic fields in broadening the heat capacity peaks and moving them to higher temperature. The adiabatic temperature changes show the point, $T_{c^{\prime}}$ although the dependence is not that of an ideal magnetic refrigerant.

Figures 91 and 92 show the contrasting behavior of $\mathrm{Er}_{0.86} \mathrm{Gd}_{0.14} \mathrm{Al}_{2}$ and Figures 93 and 94 that of $\mathrm{Er}_{0.75} \mathrm{Dy}_{0.25} \mathrm{Ni}$. Note the zero-field heat capacity anomaly for $\mathrm{Er}_{0.86} \mathrm{Gd}_{0.14} \mathrm{Al}_{2}$ stretches from about $15 \mathrm{~K}$ to over $30 \mathrm{~K}$, and the adiabatic temperature change versus temperature curve is concave downward from $15 \mathrm{~K}$ to $35 \mathrm{~K}$. Although the Curie point of $\mathrm{Er}_{0.86} \mathrm{Gd}_{0.14} \mathrm{Al}_{2}$ as observed in the magnetic susceptibility ${ }^{23}$ is about $40 \mathrm{~K}$, the temperature dependence of $\Delta \mathrm{T}_{\mathrm{s}}$ precludes its use in an AMR much above 20 $\mathrm{K}$. $\mathrm{Er}_{0.75} \mathrm{Dy}_{0.25} \mathrm{Ni}$ shows similar broadening, although not as severe as for $\mathrm{Er}_{0.86} \mathrm{Gd}_{0.14} \mathrm{Al}_{2}$.

Figures 95 and 96 show $\mathrm{C}_{\mathrm{b}}$ and $\Delta \mathrm{T}_{\mathrm{s}}$ of the disordered alloy $\mathrm{Er}_{0.8} \mathrm{La}_{0.2} \cdot$ As noted previously, the multiple magnetic transitions observed in pure Er between $19 \mathrm{~K}$ and 84 $\mathrm{K}$ have coalesced into a single ferromagnetic transition at $45 \mathrm{~K}$ for this alloy. The magnetic transition does not reflect the strongly broadened character of $\mathrm{Er}_{0.86} \mathrm{Gd}_{0.14} \mathrm{Al}_{2}$, but the decline of $\Delta \mathrm{T}_{\mathrm{s}}$ below the Curie point is slower than that of GdPd and GdNi-based materials. Magnetic hysteresis appears below $25 \mathrm{~K}$ as a splitting between the $\Delta T_{s}$ curves upon application of and removal of the magnetic field. This hysteresis will somewhat reduce the efficiency of an AMR stage operating below $25 \mathrm{~K}$. The hysteresis may be due to remnants of the antiferromagnetic transition seen in pure Er. If so, greater dilution with La should remove it, although at the expense of a slightly larger lattice heat capacity and hence a slightly smaller $\Delta \mathrm{T}_{\mathrm{s}}$.

Preliminary results on $\mathrm{GdNi}_{0.22} \mathrm{Cu}_{0.78}$ suggest that it has a similar $\Delta \mathrm{T}_{\mathrm{s}}$ to $\mathrm{Er}_{0.8}{ }^{\mathrm{La}}{ }_{0.2}$, but a smaller heat capacity.

\subsubsection{Analysis of the Magnetocaloric Data}

The heat capacity of magnetic metals can be considered as the sum of three terms: $\mathrm{C}_{1}$, the lattice heat capacity, $\mathrm{C}_{\mathrm{e}}$, the heat capacity due to conduction electrons, and $C_{m}$, the magnetic heat capacity. Only the last term is field-dependent and is involved in the magnetocaloric effects of interest here. The $\mathrm{C}_{1}$ is conventionally taken to be of the Debye form which goes as $\mathrm{AT}^{3}$ at low temperature and saturates at a constant value above the Debye temperature; $C_{e}$ is normally proportional to $\gamma \mathrm{T}$. Plots of $\mathrm{C} / \mathrm{T}$ versus $\mathrm{T}^{2}$ at low temperature should be linear with $\gamma$ as an intercept and $\mathrm{A}$ as the slope. These plots work well for non-magnetic metals but for the materials studied here, only $\gamma$ could be extracted because of curvature in the plots due to the onset of magnetic heat capacity. However, above the Curie point in zero field, the magnetic 
heat capacity becomes small, allowing fitting of the heat capacity to the full Debye form ${ }^{24}$ plus a linear term $\gamma \mathrm{T}$ determined from the low temperature $\mathrm{C} / \mathrm{T}$ plot. The resulting values of $\gamma$ and the Debye temperature are given in Table 11. The raw heat capacity data was corrected for the presence of $5 \%$ of rare-earth oxides before fitting; the $5 \%$ figure was estimated from metallography.

The fits to the lattice and electronic heat capacities may now be subtracted from the total heat capacity to extract the magnetic heat capacity. $C_{m}$ may be checked using a relation for the magnetic entropy difference $S_{m}$ between the completely ordered and disordered states of a quantized magnetic moment. The relation is $S_{m}=R \ln (2 J+1)$, where $J$ is the quantum number for the total angular momentum. The Gd-based ferromagnets studied here attain a fully-ordered state at low temperature as seen by our magnetization measurements; assuming full disorder is attained a few degrees above the Curie point, integrating $\mathrm{C} / \mathrm{T}$ from 0 to above the Curie point should give $S_{m}$ and hence an effective value of $2 J+1$. The effective values of $2 J+1$ obtained are given in Table 11, and are close to the theoretical value of 8 for Gd. For erbium compounds, the maximum theoretical value of $2 \mathrm{~J}+1$ is 16 . In $\mathrm{Er}_{0.86} \mathrm{Gd}_{0.14} \mathrm{Al}_{2}$ this value was not obtained, indicating the persistence of crystal field effects well above the Curie point; in $\mathrm{ErAl}_{2}$, the lower-lying crystal field state ${ }^{25}$ have a multiplicity of 10 , which is close to the effective value of $2 \mathrm{~J}+1$ obtained for $\mathrm{Er}_{0.86} \mathrm{Gd}_{0.14} \mathrm{Al}_{2}$. For $\mathrm{Er}_{0.8} \mathrm{La}_{0.2}$, the full theoretical value of $2 \mathrm{~J}+1$ is obtained by integrating to about $10 \mathrm{~K}$ above the Curie point; $81 \%$ of the magnetic entropy occurs below the Curie point, indicating that crystal field splittings do not appreciably reduce the magnetocaloric potential of this material. 


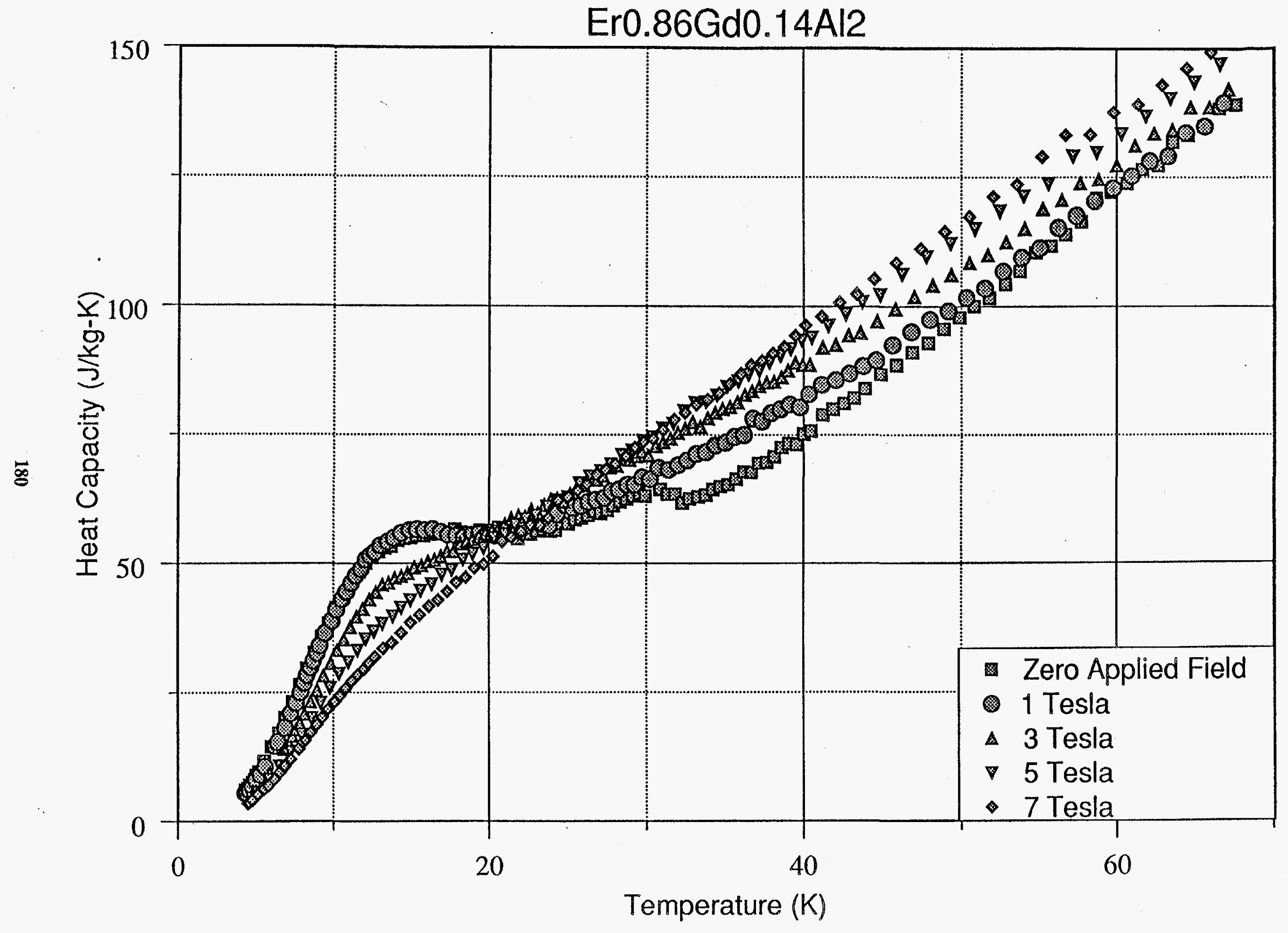

Figure 91. $C_{B}$ for $\mathrm{Er}_{\mathbf{0 . 8 6}} \mathrm{Gd}_{\mathbf{0 . 1 4}} \mathrm{Al}_{2}$

Note: Refer to the broadened ferromagnetic transition. 
Er0.86Gd0.14Al2

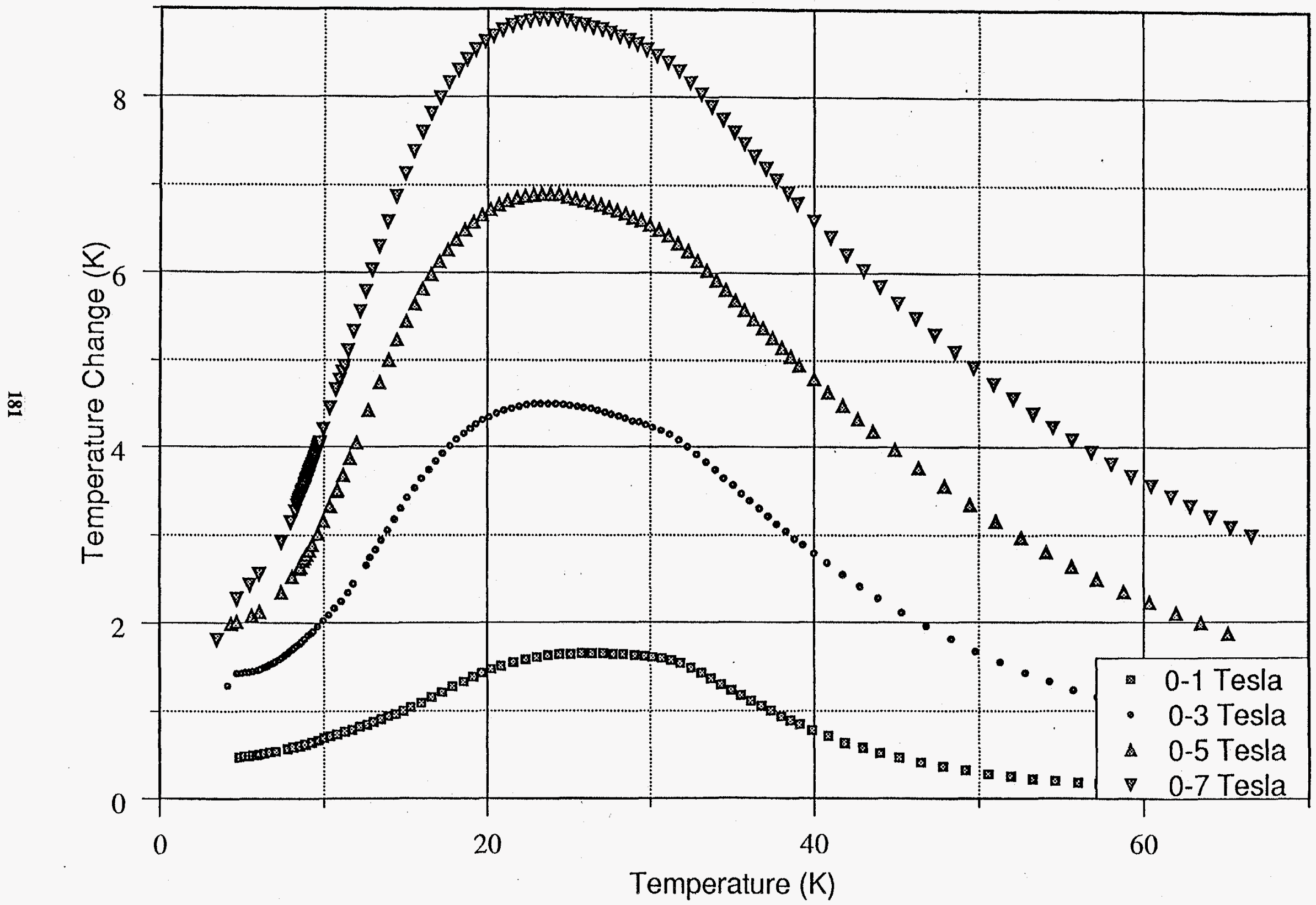

Figuire 92. $\Delta \mathrm{Ts}$ for $\mathrm{Er}_{0.86} \mathbf{G d}_{0.14} \mathrm{Al}_{2}$

Note: This was completed prior to the start of the contract. 


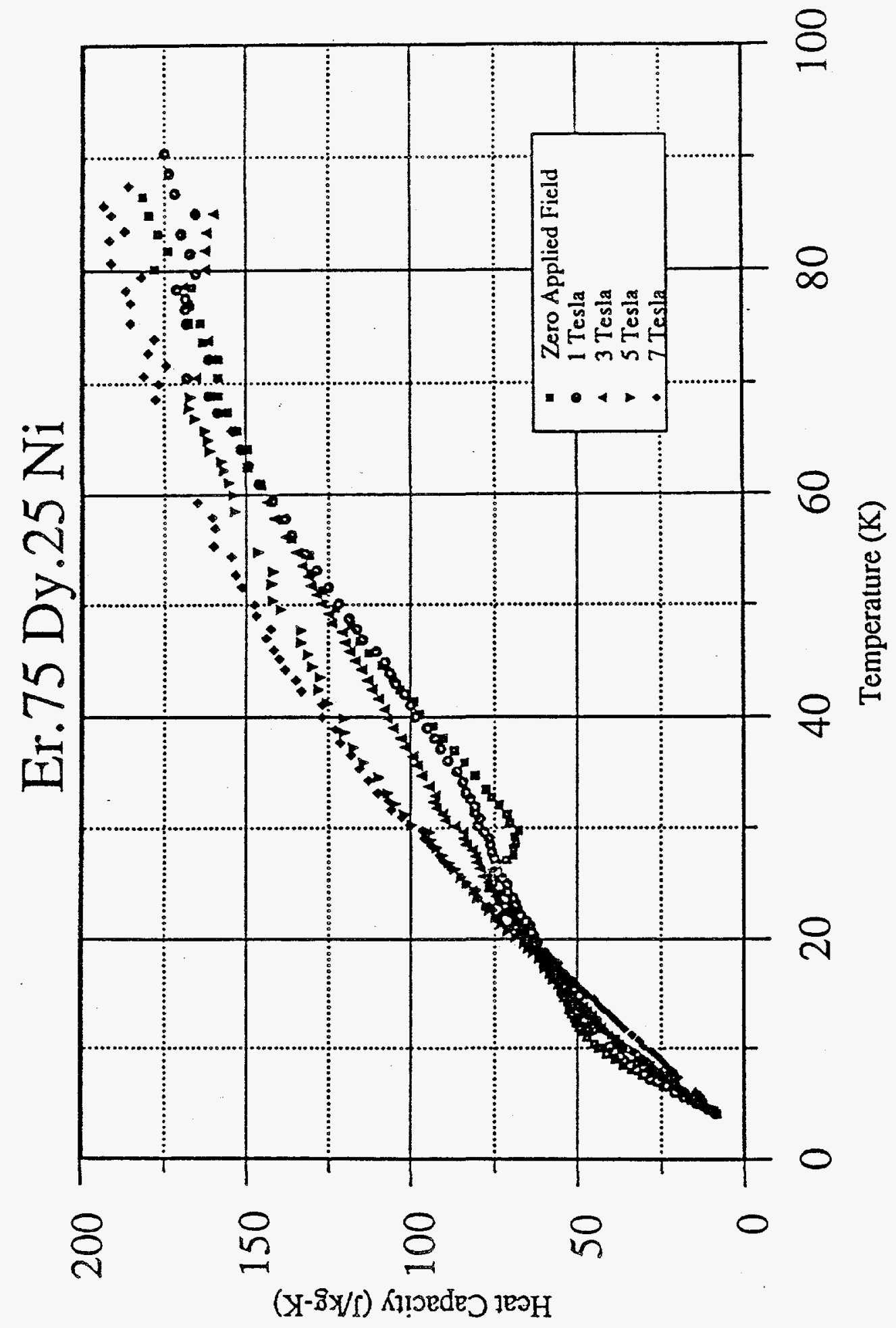

Figure 93. Measurements of $\mathrm{C}_{\mathrm{B}}$ on $\mathrm{Er}_{0.75} \mathrm{Dy}_{0.25} \mathrm{Ni}$

Note: This was completed prior to the start of the contract. 


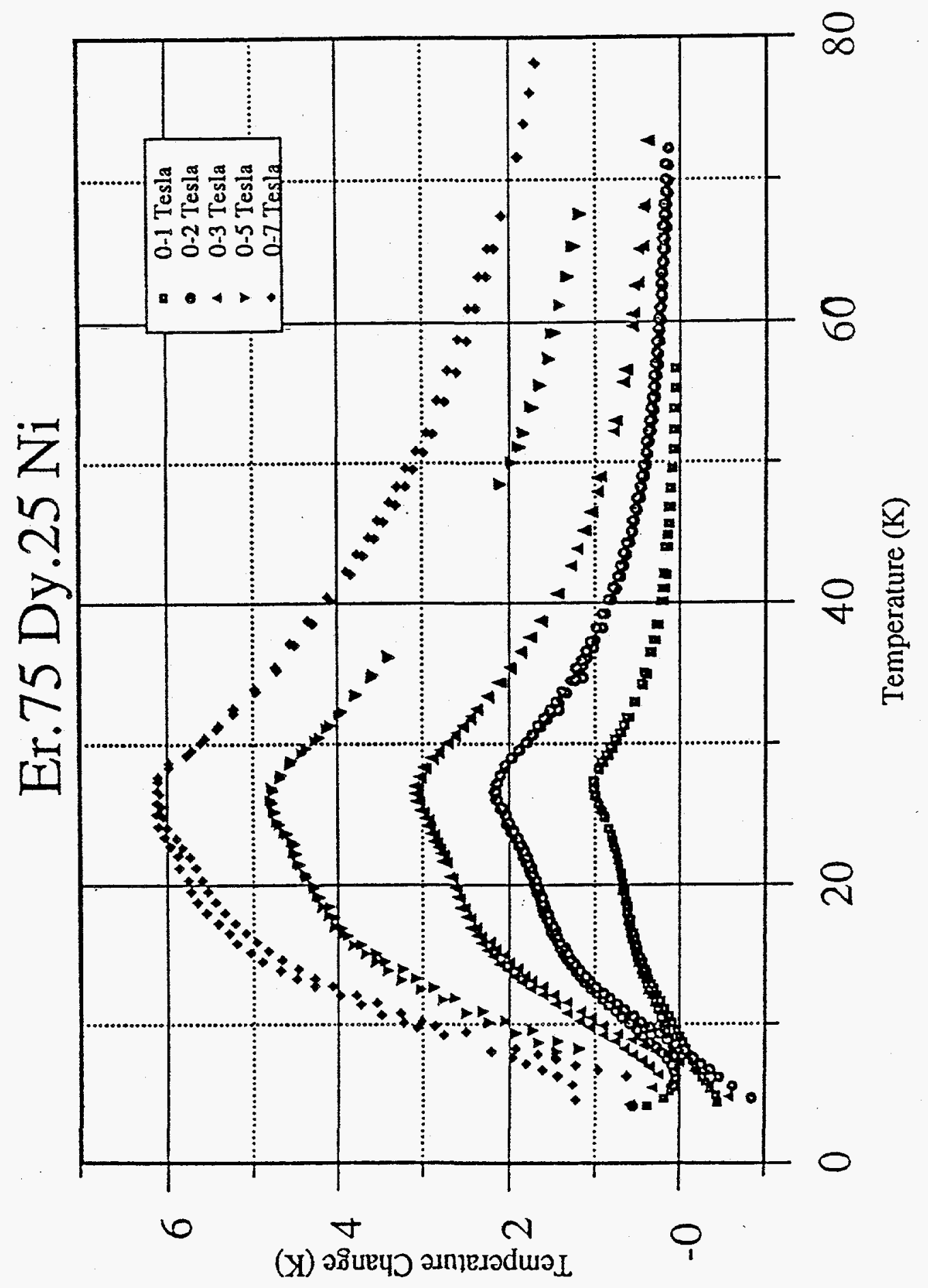

Figure 94. $\Delta \mathrm{Ts}$ for $\mathrm{Er}_{0.75} \mathrm{Dy}_{0.25} \mathrm{Ni}$ 


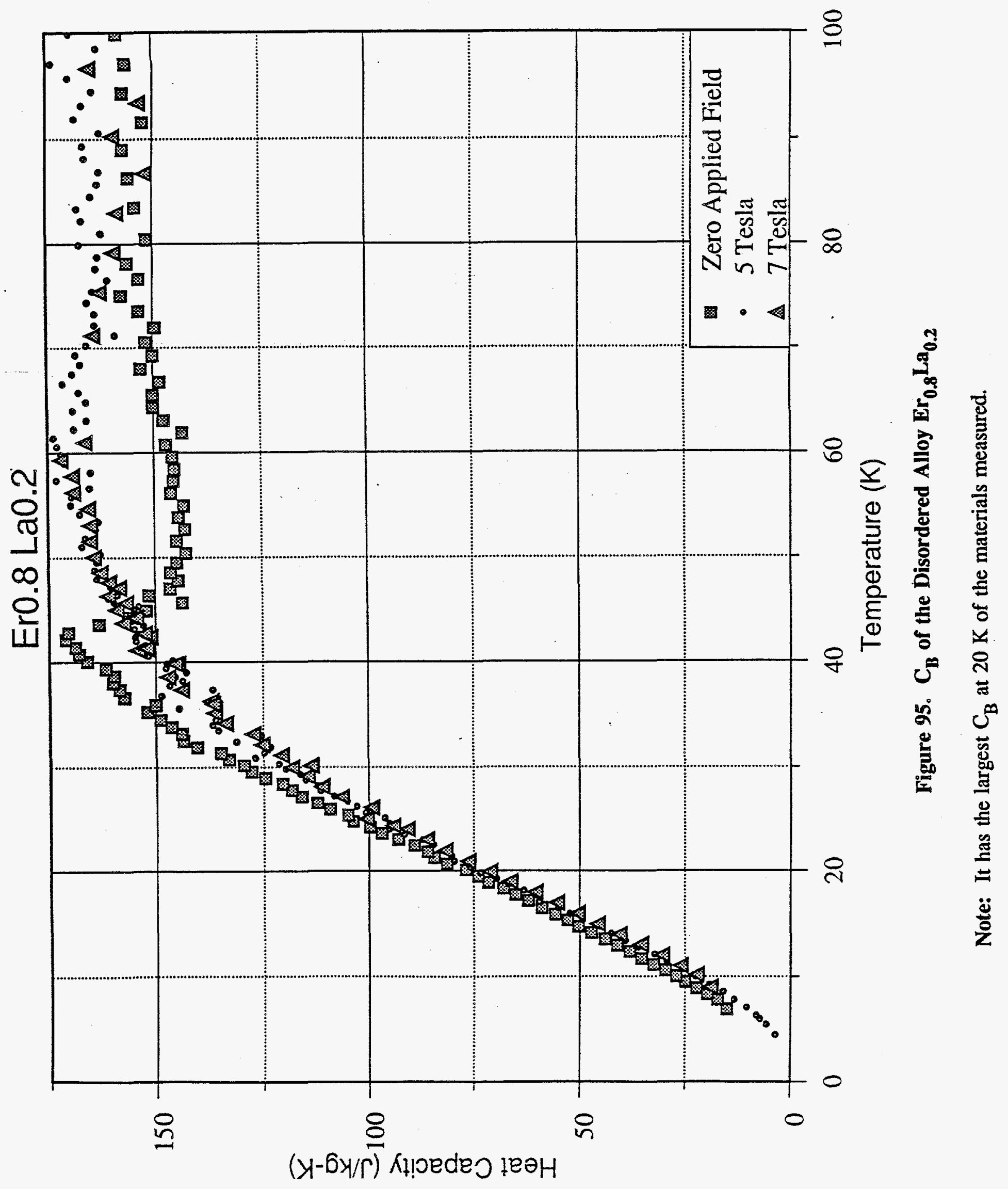




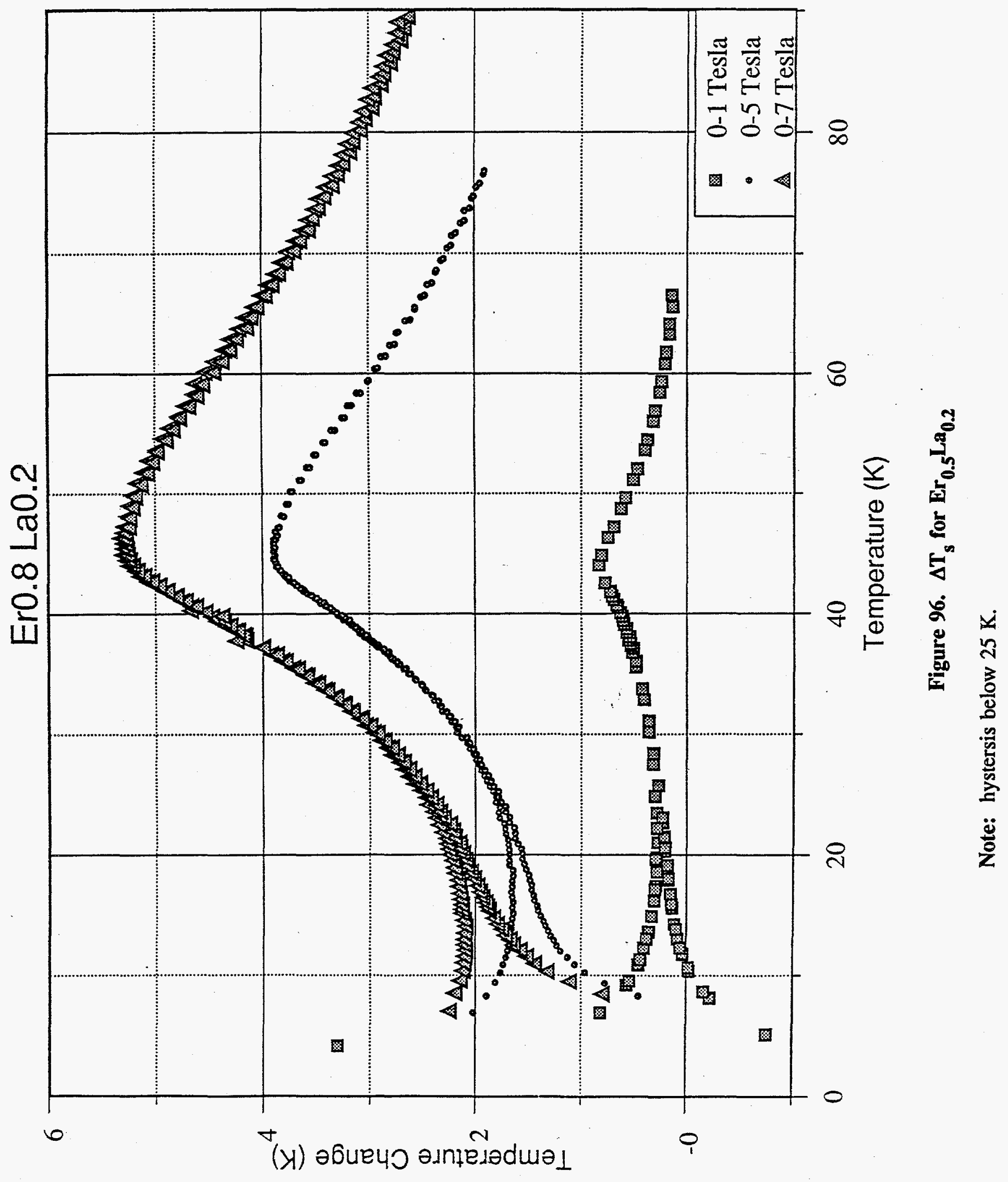


Table 11. Parameters Derived from Measured Heat Capacity of Magnetic Refrigerants

\begin{tabular}{|l|c|c|c|c|}
\hline \multicolumn{1}{|c|}{ Compound } & $\mathrm{T}_{\mathbf{C}}(\mathbf{K})$ & $\gamma\left(\mathbf{J} / \mathbf{k g - K ^ { 2 }}\right)$ & $\theta_{\mathrm{D}}(\mathbf{K})$ & $2 \mathbf{J}_{\text {eff }}+\mathbf{1}$ \\
\hline $\mathrm{GdPd}$ & 38 & $0.05 \pm 0.02$ & $185 \pm 5$ & $8.03 \pm 0.4$ \\
$\mathrm{GDPd}_{\mathbf{0 . 7 5}} \mathrm{Ni}_{\mathbf{0 . 2 5}}$ & 45 & 0.055 & 195 & 8.13 \\
$\mathrm{GdNi}^{2}$ & 70 & 0.075 & 205 & 7.73 \\
$\mathrm{GdNi}_{\mathbf{0 . 9 5}} \mathrm{Co}_{\mathbf{0 . 0 5}}$ & 81 & 0.075 & 205 & 8.53 \\
$\mathrm{GdNi}_{2}$ & 75 & 0.07 & 245 & 7.98 \\
$\mathrm{Er}_{\mathbf{0 . 8 6}} \mathrm{Gd}_{\mathbf{0 . 1 4}} \mathrm{Al}_{2}$ & 32 & 0.05 & 330 & 10.165 \\
$\mathrm{Er}_{\mathbf{0 . 8}} \mathrm{La}_{\mathbf{0 . 2}}$ & 45 & 0.032 & 160 & 16.6 \\
\hline
\end{tabular}


The value of $\mathrm{J}$ obtained from $\mathrm{S}_{\mathrm{m}}$ depended very critically on the choice of the Debye temperature; if it was chosen such that the $\mathrm{dC}_{\mathrm{m}} / \mathrm{dT}$ was zero well above $T_{c}$, consistently reasonable values of $J$ were obtained, whereas if the mean value of $C_{m}$ was made zero above $T_{c}$ with non-zero $\mathrm{dC}_{\mathrm{m}} / \mathrm{dT}$ allowed, erratic results were obtained. Part of the difficulty in fitting occurs because the $T_{c}$ 's are high enough that the region where $\mathrm{dC}_{\mathrm{l}} / \mathrm{dT}$ is large is masked, so the Debye temperature fitting is not very sensitive. Another potential problem is that the Debye form is only approximate, especially for compounds of dissimilar atoms. For non-conducting (hence $\mathrm{C}_{\mathrm{e}}=0$ ) magnetic compounds with low ordering temperatures, successful fitting has been done using a sum of two Debye functions, one ascribed to each type of atom. ${ }^{26}$ Another method used to estimate the lattice heat capacity is to use measured values for an isostructural rareearth compound ${ }^{26}$, such as LaNi for GdNi. With the exception of the dialuminides, no data in the temperature region of interest is available.

Plotting the heat capacities of the isostructural compounds GdNi, GdPd, $\mathrm{GdPd}_{0.75} \mathrm{Ni}_{0.25}$, and $\mathrm{GdNi}_{0.95} \mathrm{Co}_{0.05}$ in the reduced coordinates $\mathrm{Cm} / \mathrm{R}$ and $\mathrm{T} / \mathrm{T}_{\mathrm{c}}$ produces nearly identical curves (Figure 97), showing that the ordering phenomena are similar despite the large difference in interaction strengths. Comparing the reduced heat capacities of $\mathrm{GdNi}, \mathrm{GdNi}_{2}$, and $\mathrm{Er}_{0.86} \mathrm{Gd}_{0.14} \mathrm{Al}_{2}$ (Figure 98) shows that orderings are of diverse character. Differing temperature dependences of the orderings of GdNi and $\mathrm{GdNi}_{2}$ are not surprising considering the structures differ in symmetry, numbers of next-nearest neighbors, and so on, $\mathrm{GdNi}_{2}$ and $\mathrm{Er}_{0.86} \mathrm{Gd}_{0.14} \mathrm{Al}_{2}$ have the same structure, but the latter contains two different magnetic rare earth atoms. In the Er sites, the localized nature of the $4 \mathrm{f}$ rare-earth electrons would produce a variation in local magnetic environments and hence a broadened magnetic transition, as observed in $\mathrm{Er}_{0.86} \mathrm{Gd}_{0.14} \mathrm{Al}_{2}$ and $\mathrm{Er}_{0.75} \mathrm{Dy}_{0.25} \mathrm{Ni}$. 


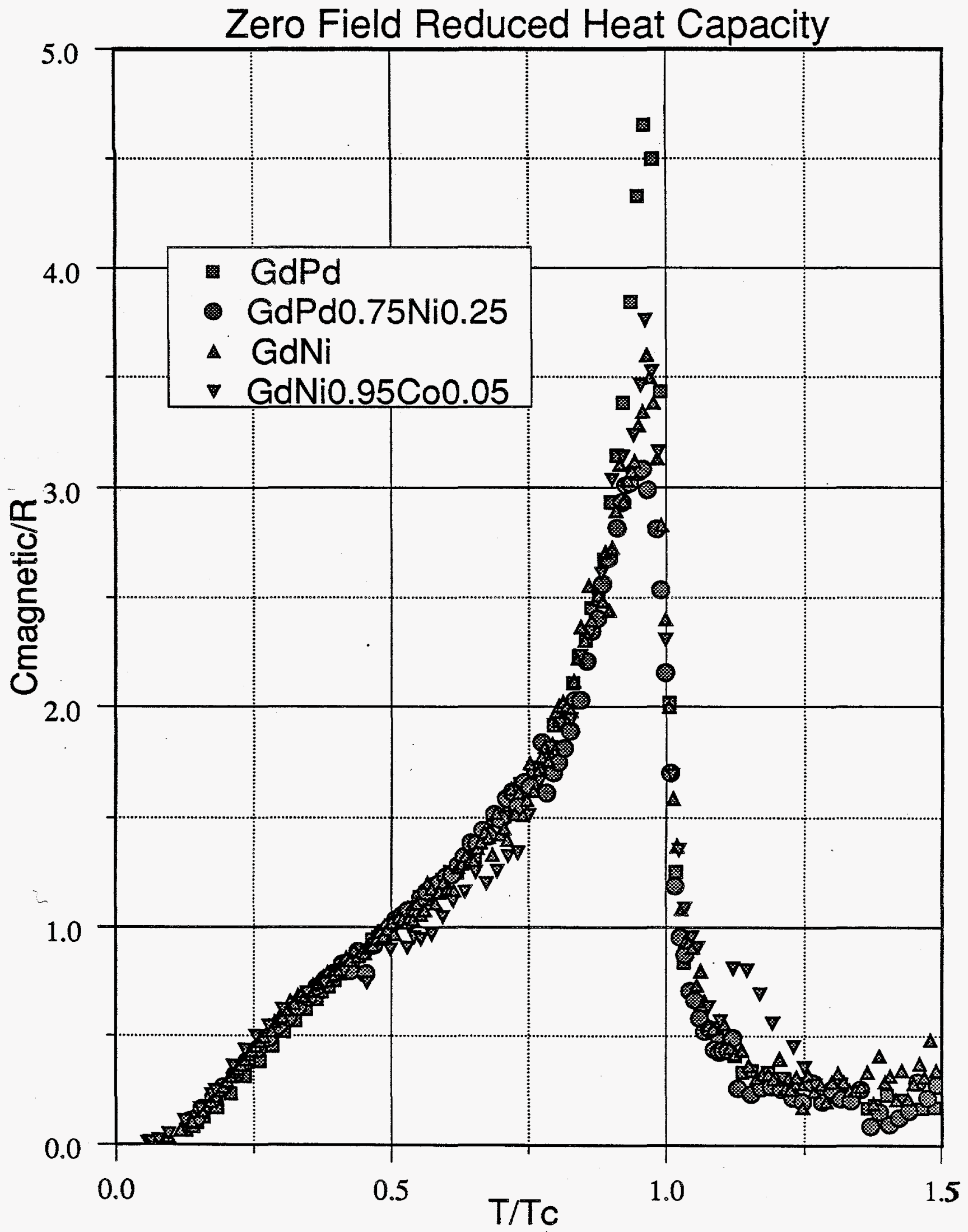

Figure 97. Reduced Magnetic Component of $C_{B}$

Note: This figure applies to some isostructural refrigerants plotted against temperature normalized to the Curie temperatures of each compound. 


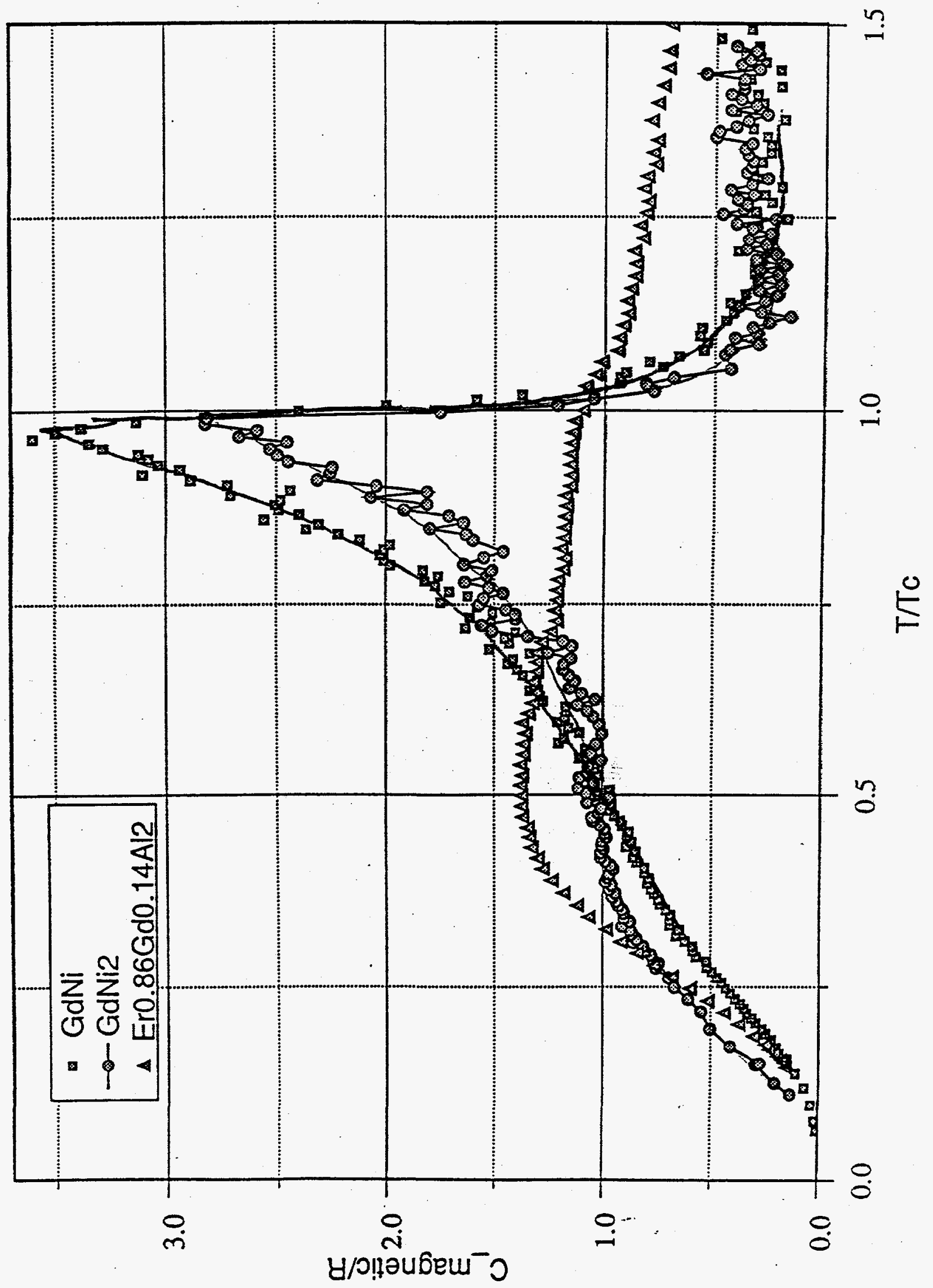

Figure 98. Reduced Magnetic $C_{B}$ Showing Dissimilar Magnetic Orderings 


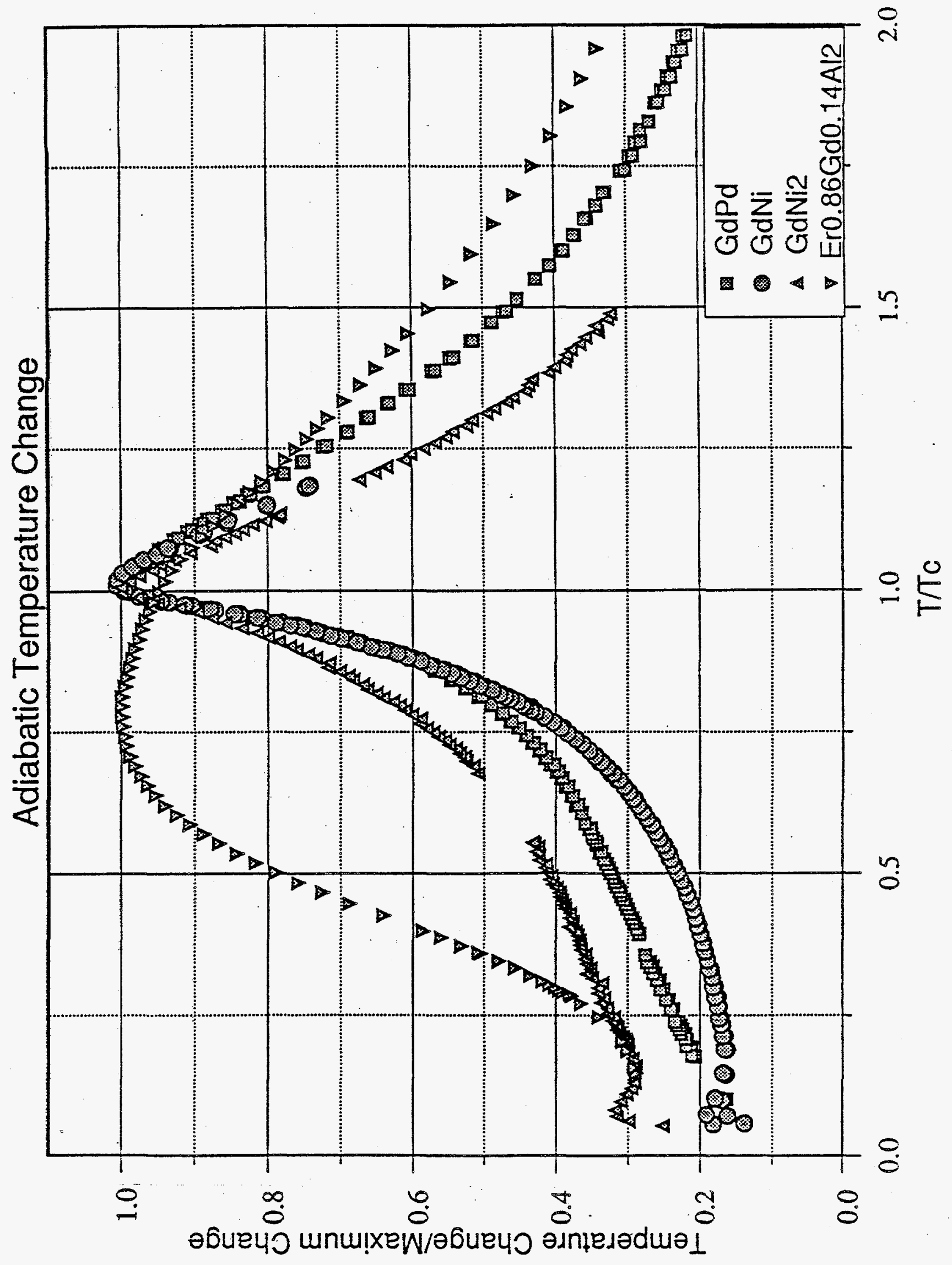

Figure 99. Comparison of $\Delta T_{s}$ for Several Magnetic Refrigerants 
Comparing $\Delta \mathrm{T}_{\mathrm{s}}$ among the materials is more difficult because the presence of the magnetic field introduces another energy, $\mu \mathrm{H}$, where $\mu$ is the atomic magnetic moment. Correcting for lattice effects is also no longer a simple matter of subtracting a lattice heat capacity. Plotting $\Delta T_{s}$ divided by its maximum value against $T / T_{c}$ (Figure 99) does show similar trends to that of Figure 86. $\Delta \mathrm{T}_{\mathrm{s}}$ of GdNi and GdPd are similar; $\mathrm{GdNi}_{2}$ is slightly broader and $\mathrm{Er}_{0.86} \mathrm{Gd}_{0.14} \mathrm{Al}_{2}$ shows a wide peak.

\subsection{Applicability of the Materials to Hydrogen Liquefaction}

From the standpoint of $20 \mathrm{~K}$ to $80 \mathrm{~K}$ AMR application, $\mathrm{GdNi}_{2}$ is an excellent material to use in an upper stage. $\Delta \mathrm{T}_{\mathrm{s}}$ is approximately linearly proportional to temperature from $40 \mathrm{~K}$ to $80 \mathrm{~K}$, and its maximal value of $0.9 \mathrm{~K}$ per Tesla allows use of reasonable magnetic fields of 5 to 7 Tesla. Hysteresis is also negligible for $\mathrm{GdNi}_{2}$ in the $40 \mathrm{~K}$ to $80 \mathrm{~K}$ region. $\mathrm{GdNi}$ and $\mathrm{GdNi}_{0.95} \mathrm{Co}_{0.05}$ have larger heat capacities and $\Delta \mathrm{T}_{\mathrm{s}}$ than $\mathrm{GdNi}_{2}$, but the rapid temperature dependence of their $\Delta \mathrm{T}_{\mathrm{s}}$ below $\mathrm{T}_{\mathrm{c}}$ makes them less suitable for use over a broad range of temperature.

GdPd or $\mathrm{GdPd}_{0.75} \mathrm{Ni}_{0.25}$ can be used in a lower stage spanning the remaining 40$45 \mathrm{~K}$ to $20 \mathrm{~K}$ region. Hysteresis is also minimal for these materials. In this temperature range, $C_{p}$ of helium decreases with increasing temperature (at $20 \mathrm{~atm}$ ) and the flow unbalance becomes significant. Hence $\Delta T_{s}$ proportional to $T$ is no longer the ideal behavior. Accounting for a flow imbalance of $13 \%$ but neglecting the temperature dependences of the heat capacity of helium and the bed and effects of a finite heat transfer coefficient between the bed and the helium, a temperature dependence of $\Delta \mathrm{T}_{\mathrm{s}}$ of the form $\Delta T_{s}=c T^{1.13}-T$ was shown to produce the highest possible efficiency, where $\mathrm{c}$ is an adjustable constant. The $\Delta \mathrm{T}_{\mathrm{s}}$ of neither $\mathrm{GdPd}$ nor $\mathrm{GdPd}_{0.75} \mathrm{Ni}_{0.25}$ fits this form very well over the entire temperature span of the lower stage (Figure 100). It remains to be determined, however, whether a material following the maximum efficiency curve will necessarily produce a device with the largest cooling power or minimum capital cost. 


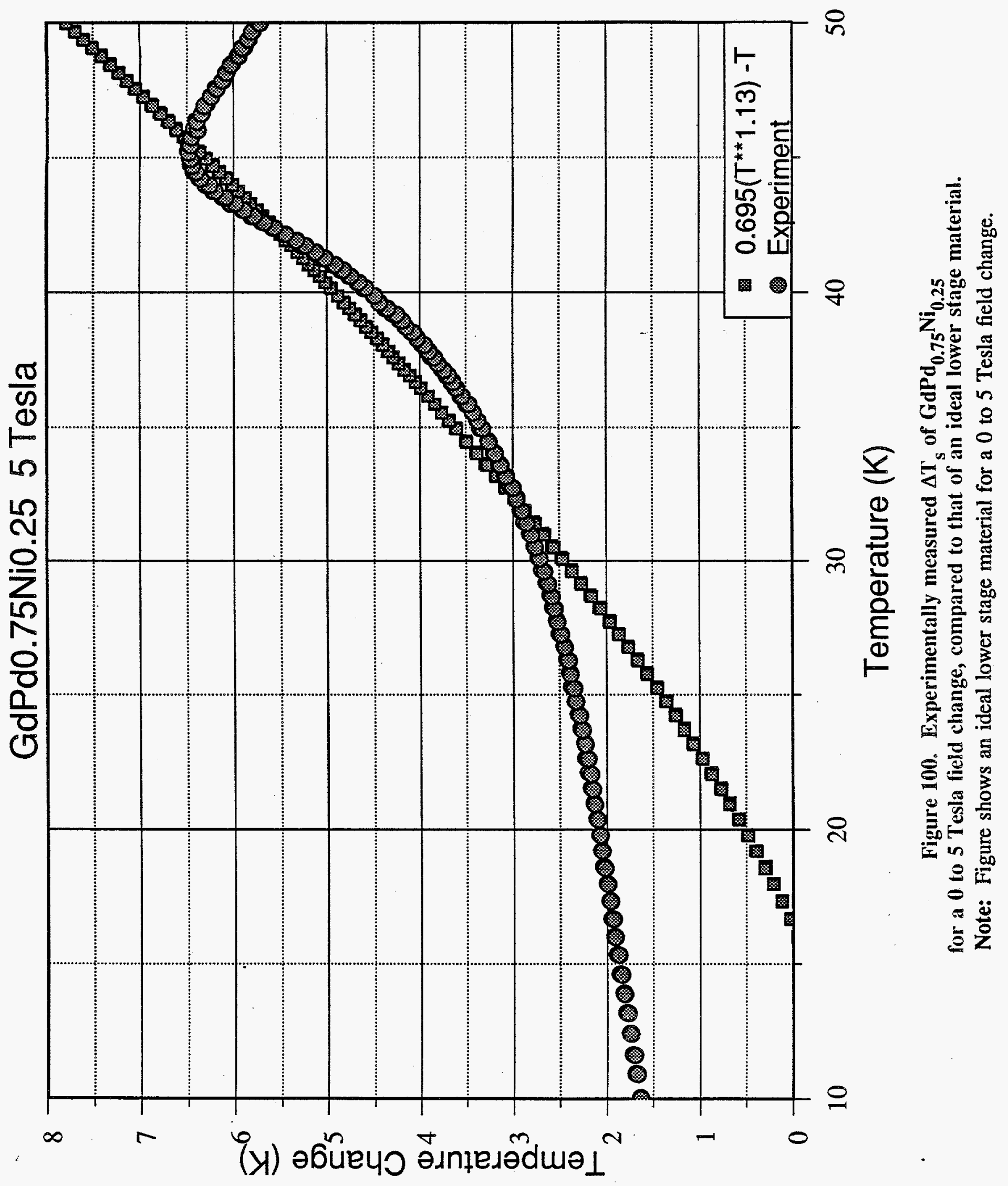




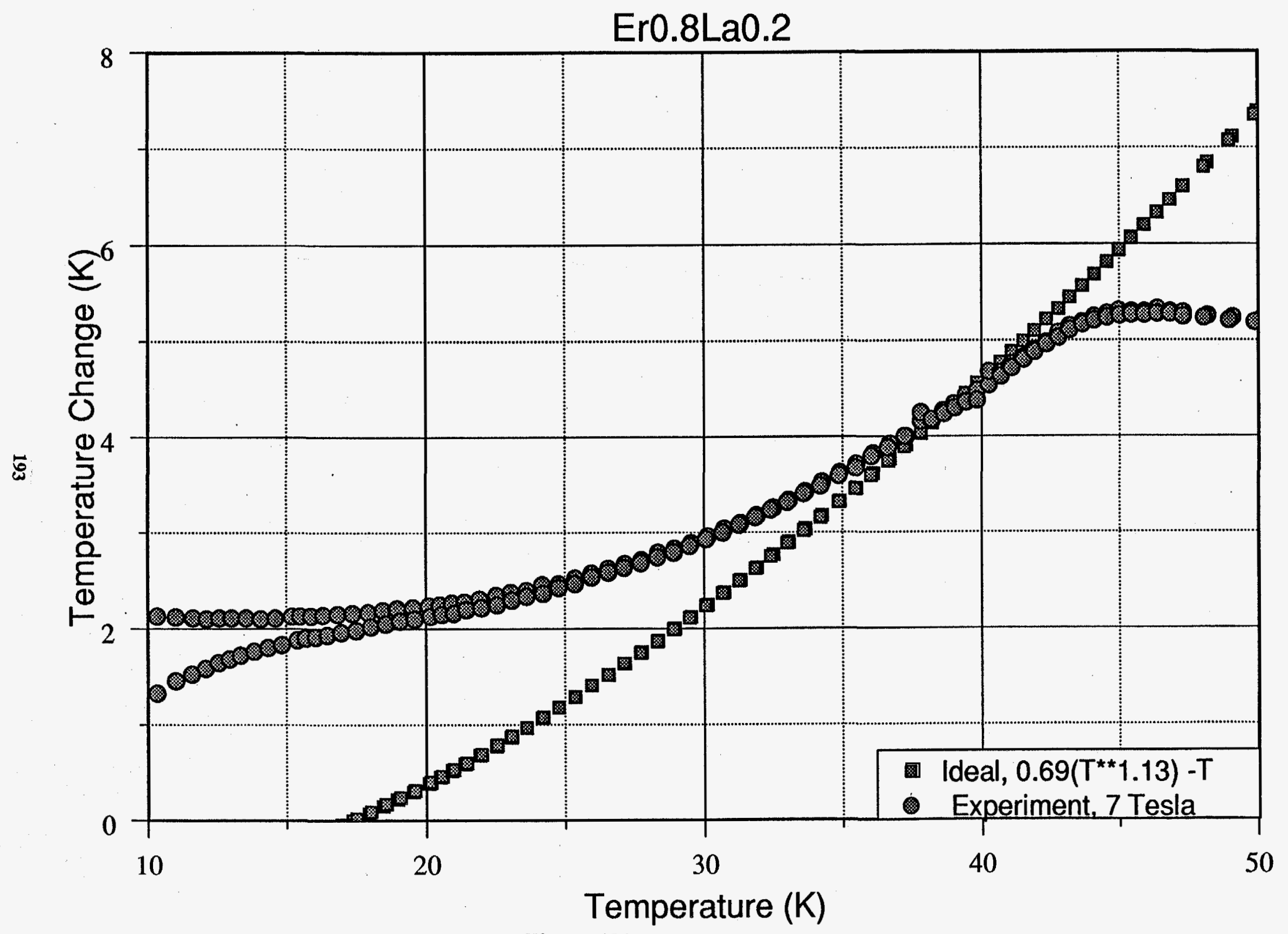

Figure 101. $\Delta \mathrm{Ts}$ for $\mathrm{Er}_{0.8} \mathrm{La}_{0.2}$ at 7 Tesla

Note: Figure information is compared to a curve which gives maximum efficiency using an ideal lower stage material. 
$\mathrm{Er}_{0.8} \mathrm{La}_{0.2}$ is also a potential lower-stage material. It has a smaller $\Delta \mathrm{T}_{\mathrm{s}}$ than GdPd, but a larger heat capacity. The $\Delta \mathrm{T}_{\mathrm{s}}$ has a different temperature dependence from the Pd compounds, but still does not fit the curve of maximum efficiency (Figure 101). The smaller magnitude of $\Delta \mathrm{T}_{\mathrm{s}}$ can be partially offset by increasing the applied field, e.g., from $5 \mathrm{~T}$ to $7 \mathrm{~T}$. $\mathrm{Er}_{0.8} \mathrm{La}_{0.2}$ uses much cheaper starting ingredients than GdPd.

$\mathrm{Er}_{0.86} \mathrm{Gd}_{0.14} \mathrm{Al}_{2}$ is only suitable for use below $20 \mathrm{~K}$ because of the broadening of its magnetic transition. It did work well in our $7 \mathrm{~K}$ to $20 \mathrm{~K}$ AMR tests.

Combining two different materials, with the part of the bed operating below $30 \mathrm{~K}$ composed of $\mathrm{Er}_{0.8} \mathrm{La}_{0.2}$ and the portion operating above $30 \mathrm{~K}$ composed of $\mathrm{GdPd}_{0.75} \mathrm{Ni}_{0.25}$, produces bed properties closer to that of the maximum efficiency curve (Figure 102). This layering technique is possible for the AMR because the various layers of the bed of a given stage do not span the entire temperature span of the stage. The temperature dependence of the maximum efficiency curve is sharper below $30 \mathrm{~K}$ than that of any of the materials studied. This is because the temperature dependence of $\Delta \mathrm{T}_{\mathrm{s}}$ is sharpest for a ferromagnet just below $\mathrm{Tc}$, and the materials studied, intended for non-layered beds, order well above $30 \mathrm{~K}$. A possible way to produce a second stage bed with properties closely approximating the maximum efficiency curve would be to use, at the high-temperature end of the bed, a ferromagnet with ordering at $45 \mathrm{~K}$, and, at the low temperature end, a ferromagnet (such as $\mathrm{HoAl}_{2}$ ) ordering at $30 \mathrm{~K}$, diluted with non-magnetic material to reduce the magnitude of $\Delta \mathrm{T}_{\mathrm{s}}$. Materials with a first order ferromagnetic transition, such as $\mathrm{ErCO}_{2}$, could also be used to obtain a particularly sharp temperature dependence of $\Delta \mathrm{T}_{\mathrm{s}}$. In summary, $\mathrm{GNi}_{2}$ and $\mathrm{GdPd}$ have suitable properties for use in AMR stages operating from $80 \mathrm{~K}$ to $40 \mathrm{~K}$ and $40 \mathrm{~K}$ to $20 \mathrm{~K}$, respectively. Further work should be pursued to characterize lower cost alternatives to GdPd.

\section{Regenerator Bed Materials Fabrication}

Fabrication of magnetic regenerator beds consistent with heat transfer and fluid flow requirements constitutes one of the key areas of technological development required for a successful prototype liquefier. The materials outlined previously with suitable magnetocaloric properties, unfortunately, also have poor mechanical properties. A successful approach to building the magnetic regenerators for the hydrogen liquefier will 1) produce material in a form capable of meeting heat transfer and fluid flow performance targets, 2) produce material capable of withstanding the AMR operating environment without degradation or attrition over an extended lifetime. In addition, a low to moderate cost technology is needed.

$\mathrm{GdNi}_{2}$, the material chosen for the liquefier prototype upper stage, is an ordered intermetallic compound with no observable range of stoichiometry. The $\mathrm{Gd}-\mathrm{Ni}$ 


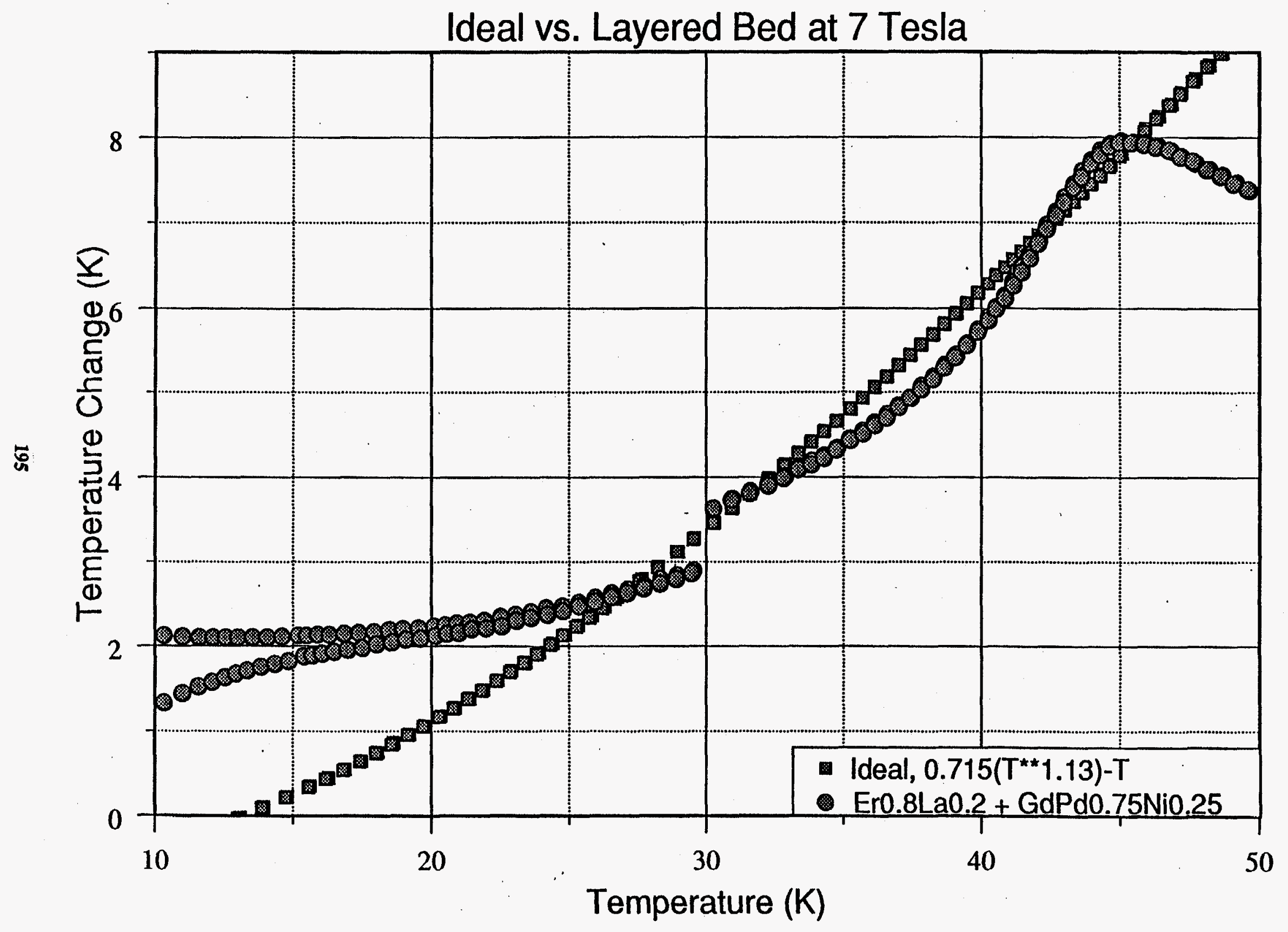

Figure 102. Layered Bed Comparison with Ideal Curve of $\Delta T_{s}$ at $7 T$

Note: Bed consists of a layer of $\mathrm{Er}_{0.8} \mathrm{La}_{0.2}$ operating between $20 \mathrm{~K}$ and $30 \mathrm{~K}$ and a layer of $\mathrm{GdPd}_{0.75} \mathrm{Ni}_{0.25}$ operating between $30 \mathrm{~K}$ and $45 \mathrm{~K}$. 
equilibrium phase diagram ${ }^{27}$ is shown in Figure 103. $\mathrm{GdNi}_{2}$ is a brittle, and, therefore, notch-sensitive material of low to moderate strength. At room temperature, it may be stored indefinitely in air. However, at elevated temperatures it reacts readily with nitrogen, oxygen, and possibly also with hydrogen or carbon monoxideconsequently all high temperature processing must be done under a high quality inert atmosphere or vacuum.

$\mathrm{GdPd}$ and $\mathrm{GdPd}_{(\mathrm{x})} \mathrm{Ni}_{(1-\mathrm{x})}$ are potential lower stage materials which are also ordered intermetallic compounds. They differ from $\mathrm{GdNi}_{2}$ in having a considerable range of allowable stoichiometry, although details of the defect chemistry are unknown. The ternary system $\mathrm{Gd}-\mathrm{Pd}-\mathrm{Ni}$ has not been investigated in detail, however, considerable (perhaps complete) solubility appears to exist between the compounds $\mathrm{GdNi}$ and GdPd. The phase diagram for the Gd - Pd system ${ }^{28}$ is shown in Figure 104. Given that the range of non-stoichiometry extends to the gadolinium-rich side of the "ideal" composition, we speculate that the predominant defects are either gadolinium interstitials or gadolinium atoms occupying palladium atomic sites. In any event, this material is far stronger than most other rare earth intermetallic compounds we have dealt with, although it is still a low ductility material. This compound appears also to be capable of dissolving small amounts of oxygen and nitrogen at temperatures near the melting point, making the quality of atmosphere during synthesis and particulation less critical. The main drawback of these materials is the high cost of palladium.

$\mathrm{Er}_{(\mathrm{x})} \mathrm{La}_{(1-\mathrm{x})}$ is a leading candidate material for the liquefier lower stage, based on acceptable magnetocaloric properties, excellent mechanical properties, and moderate cost. This alloy is a disordered solid solution of the two constituents and has strength and ductility superior to most other magnetic refrigerants. Unfortunately, $\mathrm{La}_{\text {, }}$ in its pure form, is susceptible to oxidation in air. Thus $\mathrm{Er}_{(\mathrm{x})} \mathrm{La}_{(1-\mathrm{x})}$ requires more care in synthesis to avoid excessive contamination. The alloy, once formed, appears to be stable. 


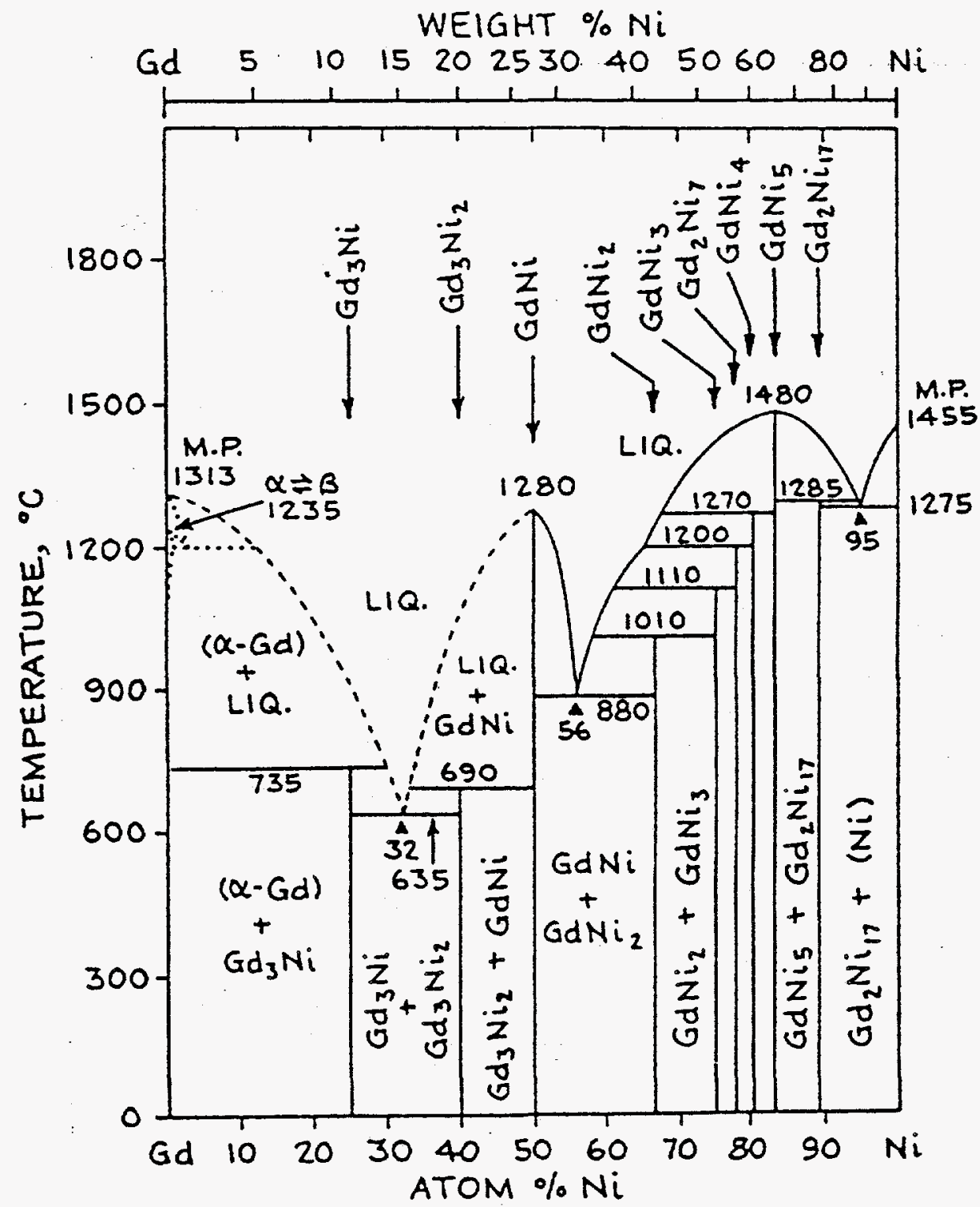

Figure 103. Phase Diagram for Gadolinium-Nickel System 


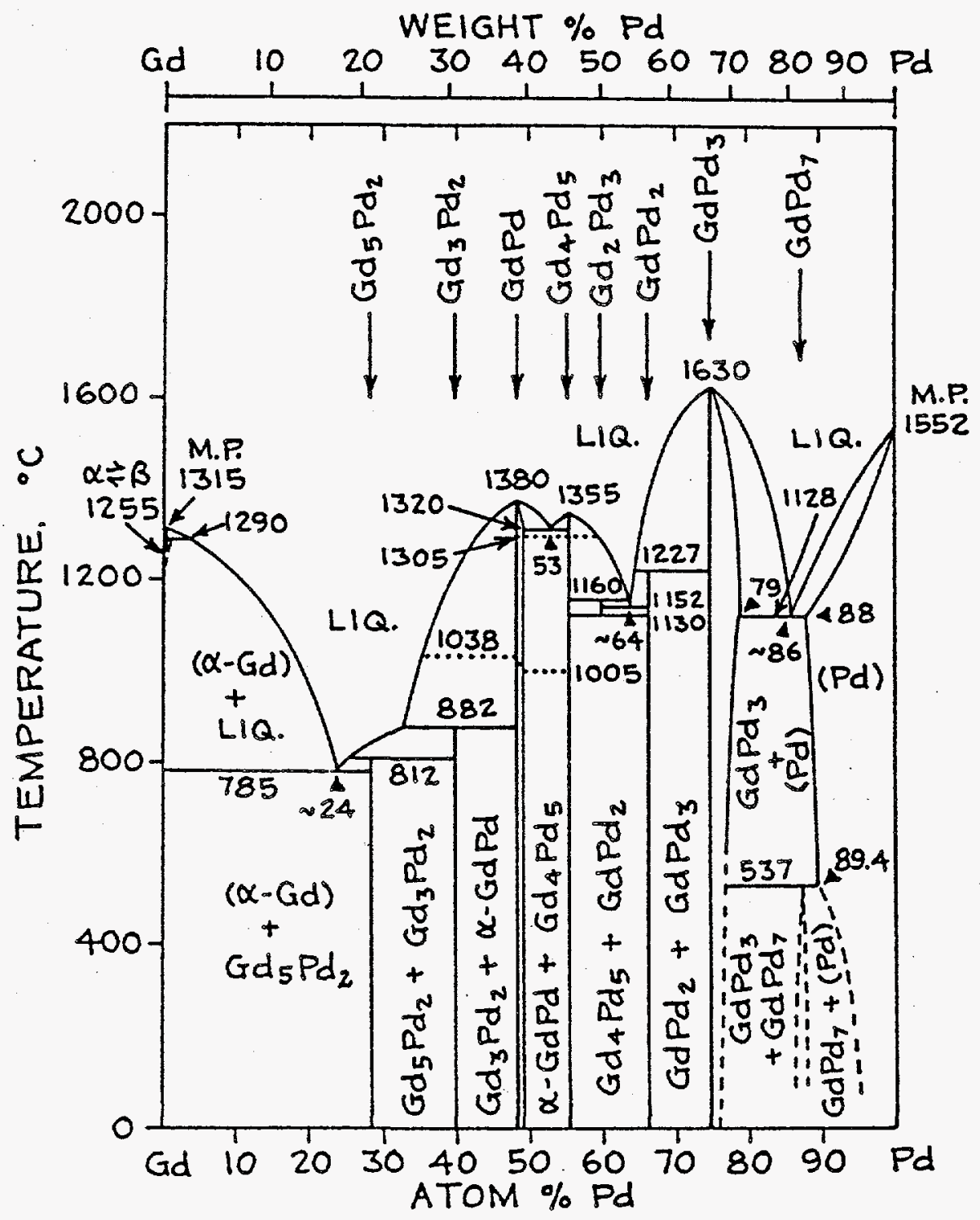

Figure 104. Phase Diagram for Gadolinium-Palladium System 


\subsection{Applicable Fabrication Techniques}

Longitudinal parallel plate, transverse perforated plate, stacked screen and packed particle bed regenerator geometries have all been successfully employed in cryocooler regenerators. Success in developing a high performance AMR is depends on the ability to fabricate one or more high performance regenerator geometries from the suitable magnetic refrigerants previously identified. Further, eventual commercial success is critically dependent on the ability to process the magnetic refrigerants and assemble high performance regenerator beds at moderate cost.

The low ductility of many suitable magnetocaloric materials effectively rules out the possibility of longitudinal parallel sheets and stacked screens. Though it may be possible to develop hot forming techniques for producing wire or thin sheets of material, the necessity of doing so under a very clean inert atmosphere environment complicates matters and ensures that the development cost would be high. Further, processing would be very material-specific, necessitating a more or less separate development cycle for each material considered. For these reasons, the screen and longitudinal parallel plate geometry have been ruled out for our present liquefier development program.

Transverse perforated plate geometries have been considered and are being studied. The effort is only exploratory at low cost due to concerns about excessive and irreducible processing costs. We have chosen to pursue actively the development of packed particle beds due to a number of factors, including 1) ability to meet targeted regenerator performance with widely varying material properties (e.g. thermal and electrical conductivity), 2) potential for processing a widely varying range of materials with little difference in technique, 3) simplicity in regenerator design and assembly and 4) the potential for low cost, high volume processing leading to a low capital cost technology.

Initial experiments with the AMR, as reported in Section 4.2 of this report, made use of granulated and screened materials; this has been sufficient to prove the viability of the AMR cycle, but problems have been experienced in some cases with settling, breakdown of material, and some loss of fine particles from the regenerator bed. In addition, the crushing process used to produce the granules has the unfortunate consequence of yielding a quantity of very small particles which are either wasted or must be reprocessed, at considerable risk of contamination. Typically, we have found that reduction of a brittle material such as $\mathrm{GdNi}_{2}$ to pass an 80 mesh (177 micron) sieve results in approximately $45 \%$ of the material breaking into granules smaller than onehalf this size and unsuitable for use in the regenerator bed. Particulate manufactured in this fashion is characterized by a large population of physical asperities-cracks, sharp corners, and irregular surfaces are the norm. Also, the particles tend to be of high aspect ratio. Qualitatively, it is easy to understand the difficulty in preparing stable regenerator beds from material of this sort: small rotational disturbances are all 
that is required to cause settling of the bed and the sharp, angular point contacts will also increase the potential for breakdown or wear.

Feasible techniques for producing spherical metal alloy particles were determined by a review of the literature and by consultation with experts at Ames Laboratory, David Taylor Research Center, Rensselaer Polytechnic Institute, University of Wisconsin, and several commercial vendors. The size range of interest for this application, 150 microns diameter, falls in a rather awkward gap between established processes. On the one hand, ball bearings and lead shot are routinely manufactured by welldeveloped processes in sizes larger than about $1 \mathrm{~mm}$, and, on the other, well-developed processes for producing bulk powders for the powder metallurgy industry concentrate on much finer products, typically 30 microns and finer. The latter technologies are summarized in Volume 7 of the ASM Metals Handbook. ${ }^{28}$

Other researchers have reported results on a closely related fabrication problem: production of 150 - 300 micron spherical particles of $\mathrm{Er}_{3} \mathrm{Ni}$ for use in enhanced performance Gifford-McMahon cryocoolers operating in the vicinity of $4 \mathrm{~K}$. Both high pressure gas atomization ${ }^{29}$ and rotating disk atomization ${ }^{30}$ have been used with a certain degree of success. However, low yields, porous particles, and various other problems have been encountered.

The technique we have chosen to pursue is based upon the natural breakup of a laminar fluid jet as described by Rayleigh. ${ }^{31}$ Although the original work was aimed at understanding the breakup of water jets, the general principles apply equally well to molten metal streams. The key phenomenon is that a laminar circular jet, forming by the escape of a liquid through a nozzle, is unstable against the formation of oscillations in its diameter at certain frequencies. An oscillation, started by any random fluctuation, continues to grow until the jet pinches off into separate liquid droplets. These droplets become spherical to minimize surface energy. The droplets cool in free-fall by radiation and convection to a surrounding inert atmosphere. Assuming the droplets solidify before coalescing or hitting a solid surface, highly spherical and void-free particles are obtained.

Figure 105 is a photograph of the apparatus and Figure 106 is a schematic of the system. Previously alloyed magnetic material is loaded into the crucible, the system is evacuated and back-filled with helium, the magnetic material is heated and melted via the induction coil and the stream is formed by a pressure difference applied across the orifice. Processed material is collected in the container at the bottom of the tower. Figure 107 shows the area of the induction coil and crucible in operation. Also visible is a stream of molten $\mathrm{GdNi}_{2}$ exiting the nozzle at ${ }^{\sim} 1550^{\circ} \mathrm{C}$.

Figure 108 shows $\mathrm{GdNi}_{2}$ particles as produced by this technique. Similar results have been obtained with GdPd and other rare earth alloys. At present, we are allowing the molten metal stream to break up under its own natural instability. While this 
appears satisfactory in many respects, we have found that the expected Rayleigh correlation between jet (nozzle) diameter and mean particle size does not hold in this system. Thus far, we have consistently achieved a mean particle size of three - four times the orifice diameter, where the Rayleigh theory predicts approximately a ratio of two.

Originally, this process was conceived for producing particulate in the $300-500$ micron size range using an orifice of around 150 to 250 microns. It was felt that 1) this orifice size range could be reliably obtained in the tungsten nozzle material and 2) that the molten metal could be ejected through this size orifice with relative ease. These two estimates have been proven accurate. Unfortunately, we have found that the particles produced by this setup are somewhat larger than expected and simultaneously, further modeling has shown that smaller (150 micron) particles are clearly advantageous. Efforts to produce smaller orifices have been successful; however, experience has shown that atomization through orifice sizes below about 125 microns is unreliable due to nozzle plugging - probably by refractory oxide impurities.

Development of a reliable, high yield technique for the production of tens of kilograms of material in the 150 micron size range is an integral and essential part of the second phase of this work. There are several backup approaches to this problem. For production of GdPd or ErLa particles for the lower stage, the rotating anode process may be applicable. This process has been used to produce neodymium spheres for the regenerator of a G-M cryocooler. We have discussed this approach with Nuclear Metals, Inc. of Concord, MA. This process is not suitable for production of $\mathrm{GdNi}_{2}$ for the upper stage. $\mathrm{GdNi}_{2}$, however, is readily crushed to produce irregular particles. Careful packing of these particles may yield a bed with sufficient durability for the testing durations contemplated for the tenth-scale device. Another longer-term solution is to develop rotating disk centrifugal atomization. ${ }^{30}$ This approach, traditionally used to produce powder for the powder metallurgy industry, may prove extensible to the manufacture of $75-300 \mu \mathrm{m}$ spherical particulate. 


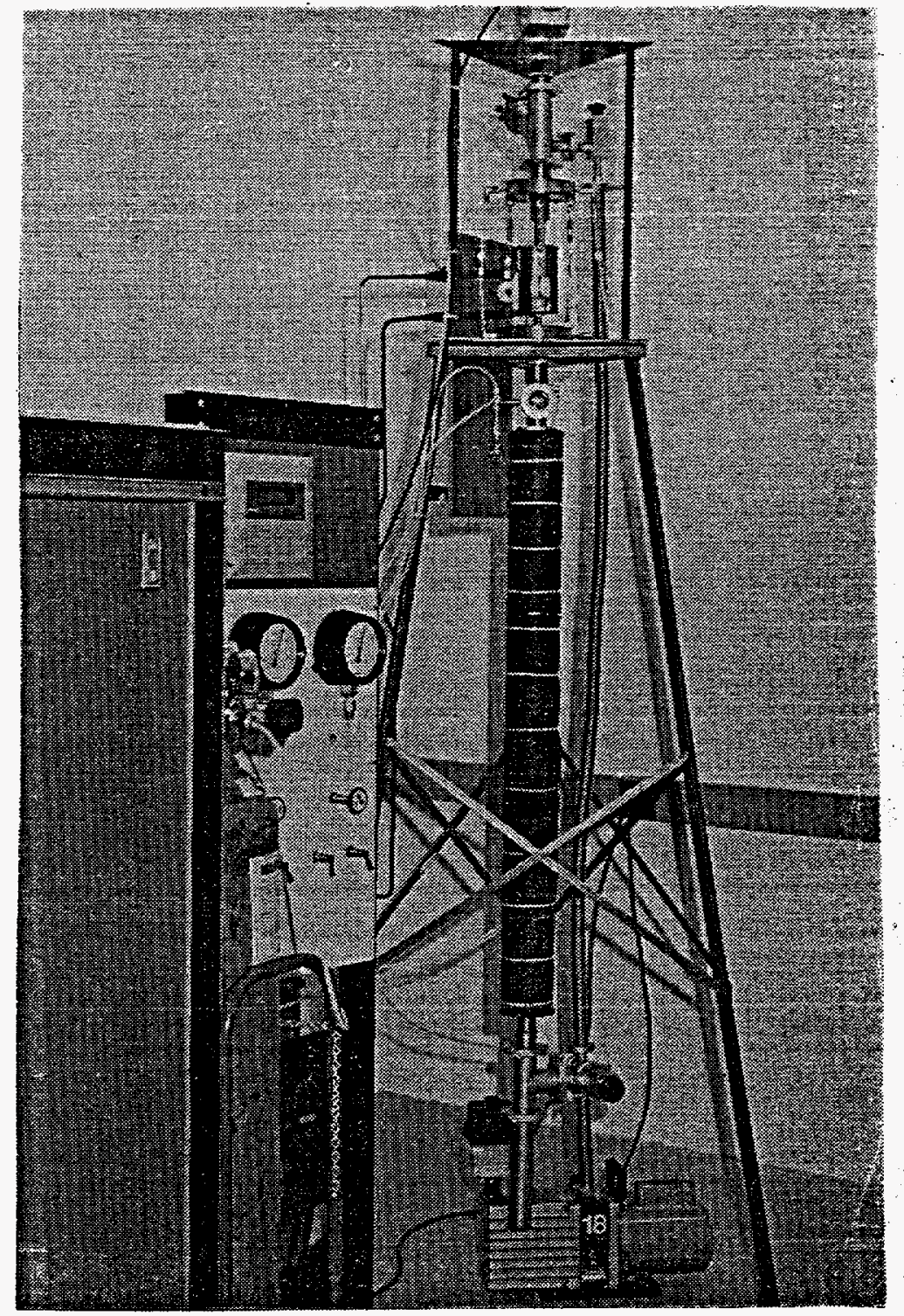

Figure 105. The ACA Particulation Apparatus

Note: The induction power supply is at far left and the crucible and tower assembly is at the right. 


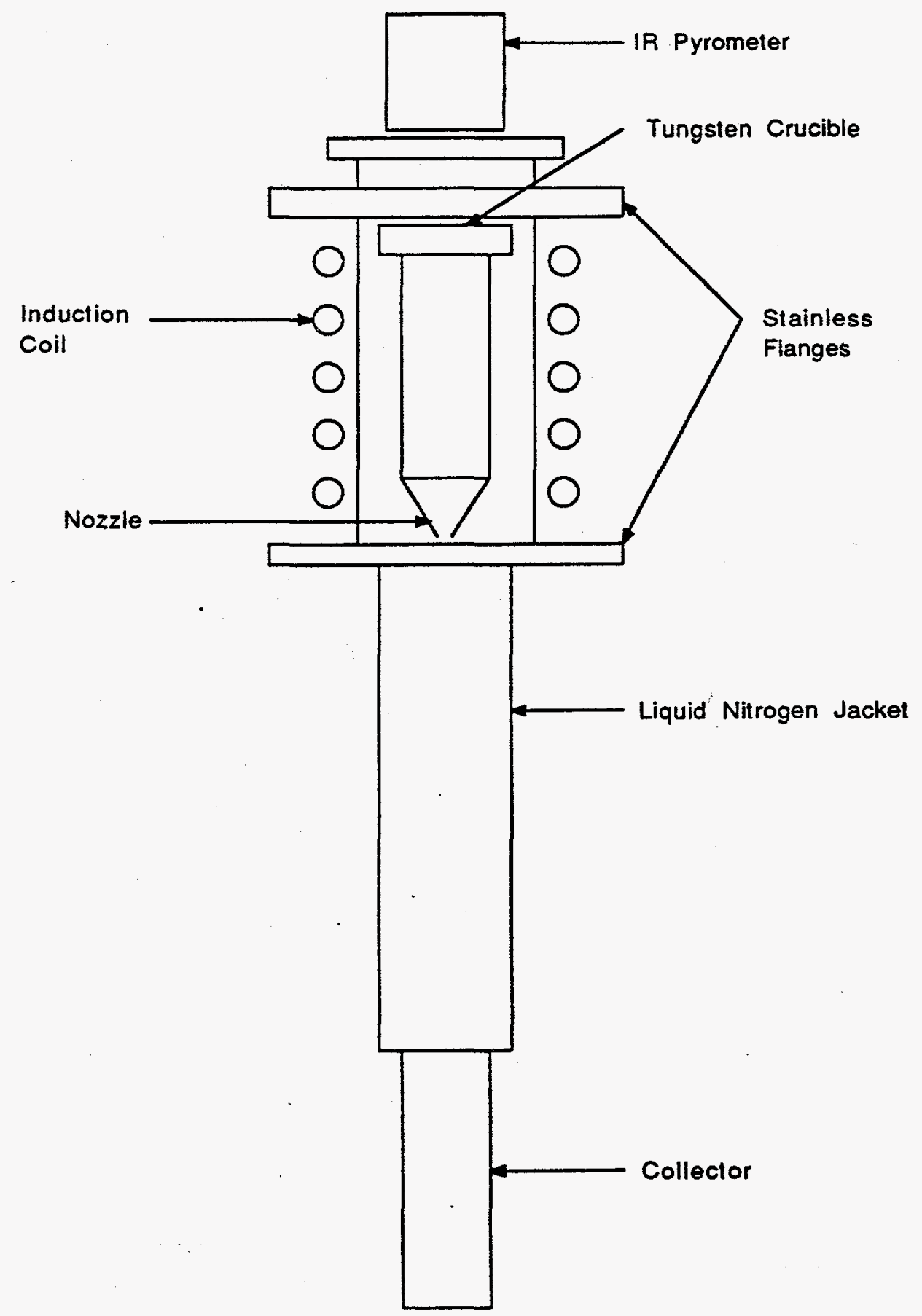

Figure 106. ACA Particulation Apparatus Schematic 


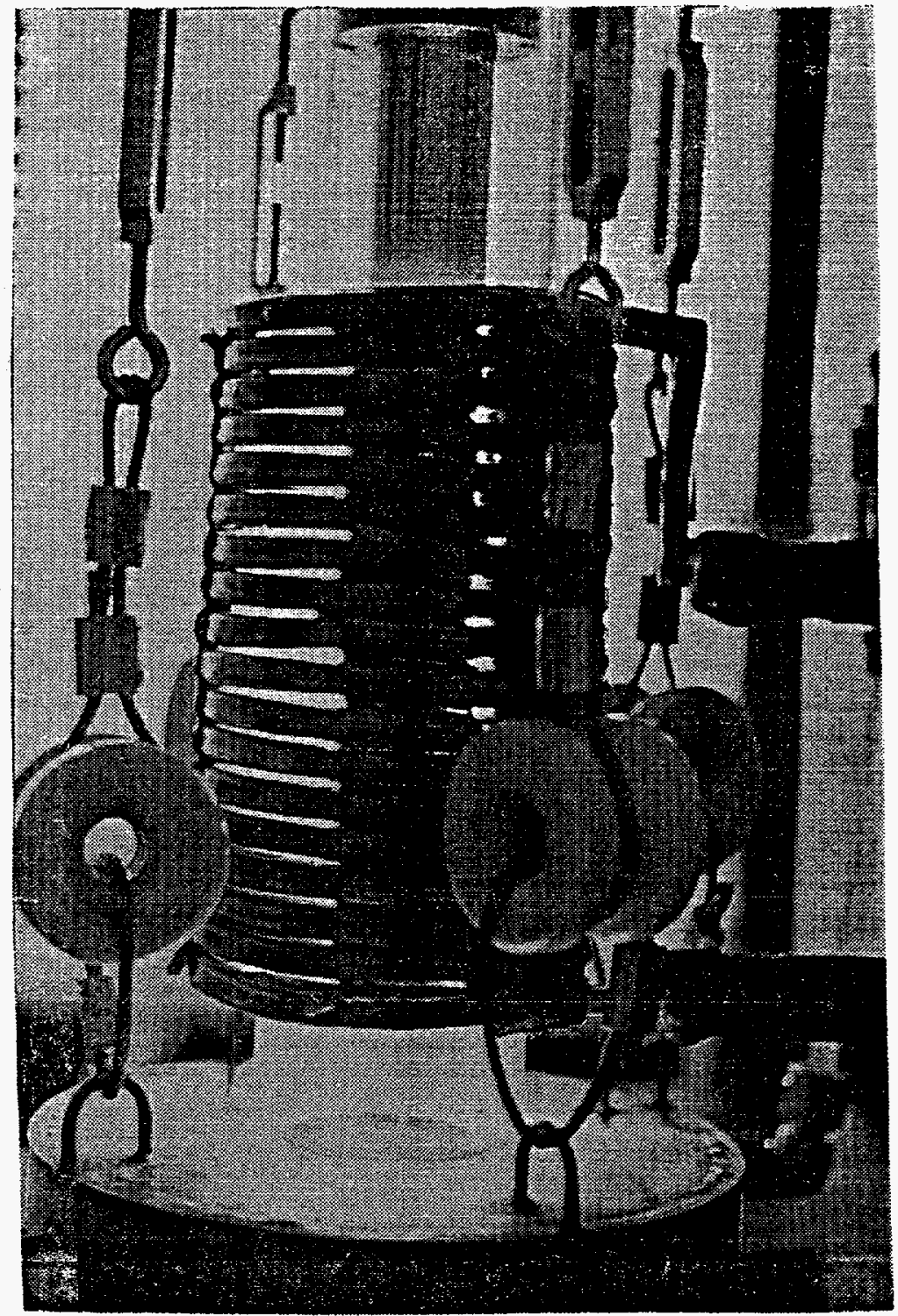

Figure 107. Induction Coil and Crucible of Particulation Apparatus

Note: A stream of molten $\mathrm{GdNi}_{2}$ is visible as a white line in the lower center. 


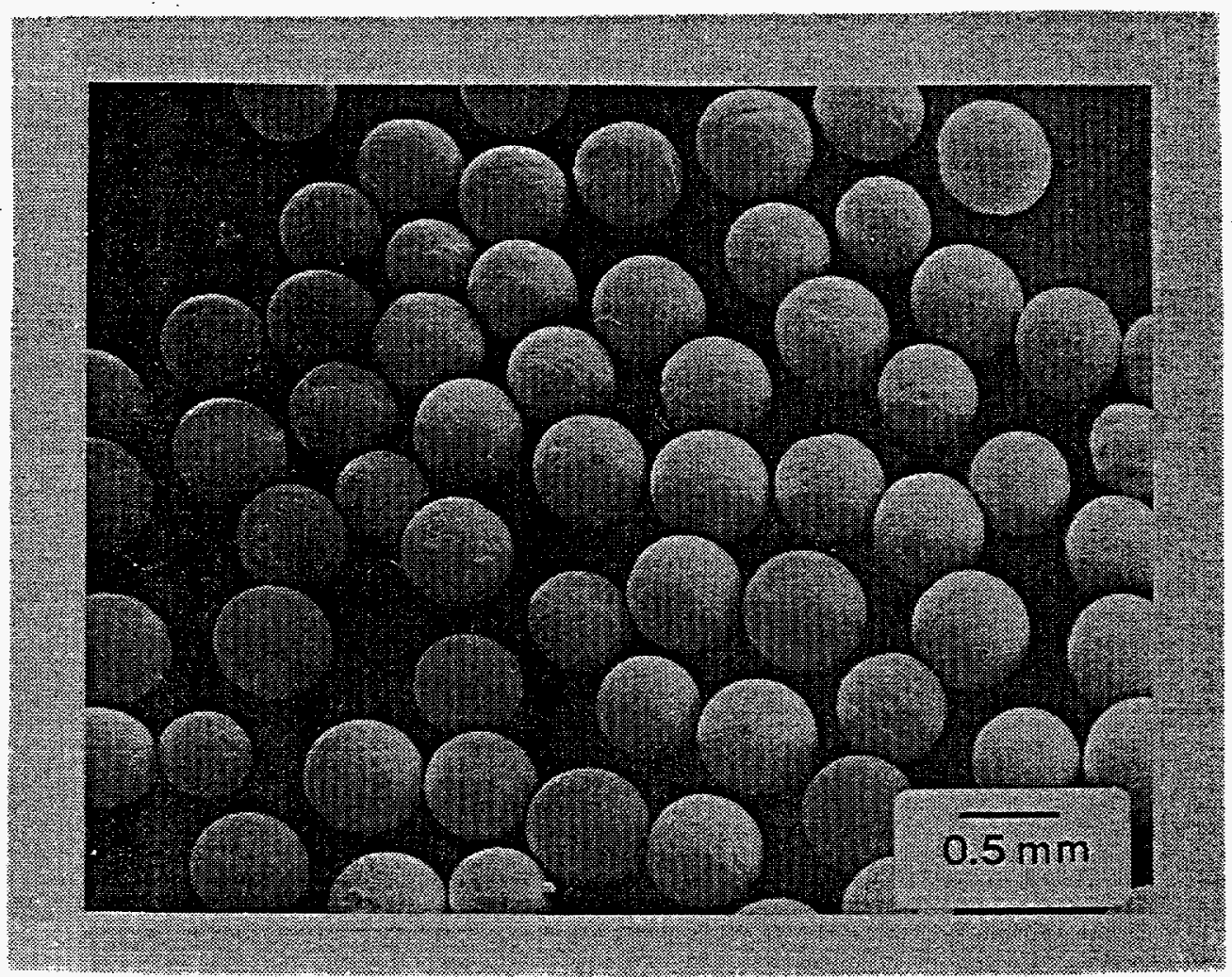

Figure 108. $\mathrm{GdNi}_{2}$ Spherical Particles Produced by Particulation Device 


\section{REFERENCES}

1. C. B. Zimm, J.A. Barclay, and W.R. Johanson et al,, Low hysteresis materials for magnetic refrigeration: $\mathrm{Gd}_{1-\mathrm{x}} \mathrm{Er}_{\mathrm{x}} \mathrm{Al}_{2}{ }^{\mathrm{a}}$, in: "J. Appl. Physics," Vol. 55, American Institute of Physics, 15 March (1984), pp. 2609-2610.

2. C.B. Zimm et al., Materials for regenerative magnetic cooling spanning $20 \mathrm{~K}$ to 80 $\mathrm{K}$, presented at the Cryogenic Engineering Conference, 11 June 1991. (to be published in "Advances in Cryogenic Engineering," Plenum Press, New York).

3. Roy E. Kannady, Jr., "Martin Marietta Interactive Thermal Analysis System" Martin Marietta Corporation, Denver, Colorado (1976).

4. F. W. Schmidt and A. J. Willmott, Thermal energy storage and regeneration, McGraw-Hill, (1981).

5. W.H. Press, B.P. Flannery, S.A. Teukolsky, V.T. Vetterling, Numerical recipes, Cambridge University Press, (1986).

6. S. Whitaker, Forced convection heat transfer correlations for flow in pipes, past flat plates, single cylinders, single spheres, and for flow in packed beds and tube bundles, in: "AIChE Journal," Vol. 18, No. 2 (1972).

7. R. D. McCarty, Thermophysical properties of helium-4 from 2 to $1500 \mathrm{~K}$ with pressures to 1000 atmospheres, "NBS technical note 631," (1972).

8. I. F. Macdonald, et al., Flow through porous media - the ergun equation revisited, Ind. Eng. Chem. Fundam., Vol. 18, (1970), pp. 199-208.

9. G. S. G. Beveridge and D. P. Haughey, Axial heat transfer in packed beds: Stagnant beds between 20 and 750C, in: "Int. J. Heat Mass Transfer," Vol. 14, (1971), pp. 1093-1113.

10. S. Sarangi and H. S. Baral, Effects of axial conduction in the fluid on cryogenic regenerator performance, Cryog., 27 (1986).

11. A.J. DeGregoria et al., Test Results of an Active Magnetic Regenerative Refrigerator, Cryogenic Engineering Conference, Huntsville, AL, June 11-14, 1991.

12. J. A. Barclay, The theory of an active magnetic regenerative refrigerator, in: "Proc. of the 2nd Biennial Conf. on Refrigeration for Cryo. Sensors and Electronic Systems," NASA Goddard Space Flight Center, Greenbelt, MD (1982).

13. J. A. Barclay and W. A. Steyert, Active Magnetic Regenerator, US Patent 4,332,135, (1 June, 1982). 
14. C. R. Cross et al., Optimal temperature entropy curves for magnetic refrigeration, in: "Advances in Cryogenic Engineering," Vol. 33, Plenum Press, New York (1988), p. 767.

15. L.G. Rubin et al., Some practical solutions to measurement problems encountered at low temperatures and high magnetic fields, in: "Advances in Cryogenic Engineering," Vol. 31, Plenum Press, New York (1986), p.1221.

16. Q. Li et al., Thick film chip resistors for use as low temperature thermometers, in: "Cryogenics," Vol. 26, (1986), p.467.

17. M. Ogawa, R. Li and T. Hashimoto, Cryogenics 31:405 (1991).

18. C. B. Zimm et al., in: "Advances in Cryogenic Engineering," Vol. 36, Plenum Press, New York (1990), p. 763.

19. Y. Y. Pan et al., Acta Phys. Sinica 35:677 (1986).

20. C. B. Zimm et al., in: "Proc. 5th Int. Cryocooler Conf., "Universal Technology Corporation," Dayton, Ohio (1988), p.49.

21. C. B. Zimm et al., in: "Advances in Cryogenic Engineering," Vol. 33, Plenum Press, New York, (1988), p.791.

22. J. A. Barclay, W. C. Overton, Jr., and C. B. Zimm, in: "Proc. LT-17," Elsevier Science Publishers, Amsterdam (1984) p. AL13.

23. A. Chelkowski, E. Talik, and G. Wnetrzak, J. Phys. F 13:483 (1983).

24. N. W. Ashcroft and N. D. Mermin, "Solid State Physics," Holt, Rinehart and Winston, New York (1976), p. 461.

25. W. E. Wallace, "Rare Earth Intermetallic Compounds," Academic Press, New York (1973), p. 43.

26. J. A. Hofmann et al., J. Phys. Chem. Solids 1:45 (1956).

27. W. G. Moffatt, "The Handbook of Binary Phase Diagrams," Genium Publishing Corporation, New York (1976, with quarterly updates).

28. "Metals Handbook, Ninth Edition, Volume 7: Powder Metallurgy," E. Klar, ed., American Society for Metals, Metals Park, Ohio (1984).

29. L. F. Aprigliano, L O'Connor, G. Green, and J. Chafe, given at the Cryogenic Engineering Conference, 11 June 1991. (to be published in Advances in Cryogenic 
Engineering," Plenum Press, New York).

30. M. Sahashi, et al., in: "Advances in Cryogenic Engineering," Vol. 35b, Plenum Press, New York, (1990) p. 1175.

31. J. W. Strutt (baron Raleigh), "The Theory of Sound," 2nd edition, Macmillan and Co., London, Chapter 20 (1894). 
Appendix A

Bed Assembly/Support Structure

Load Interaction Summary 


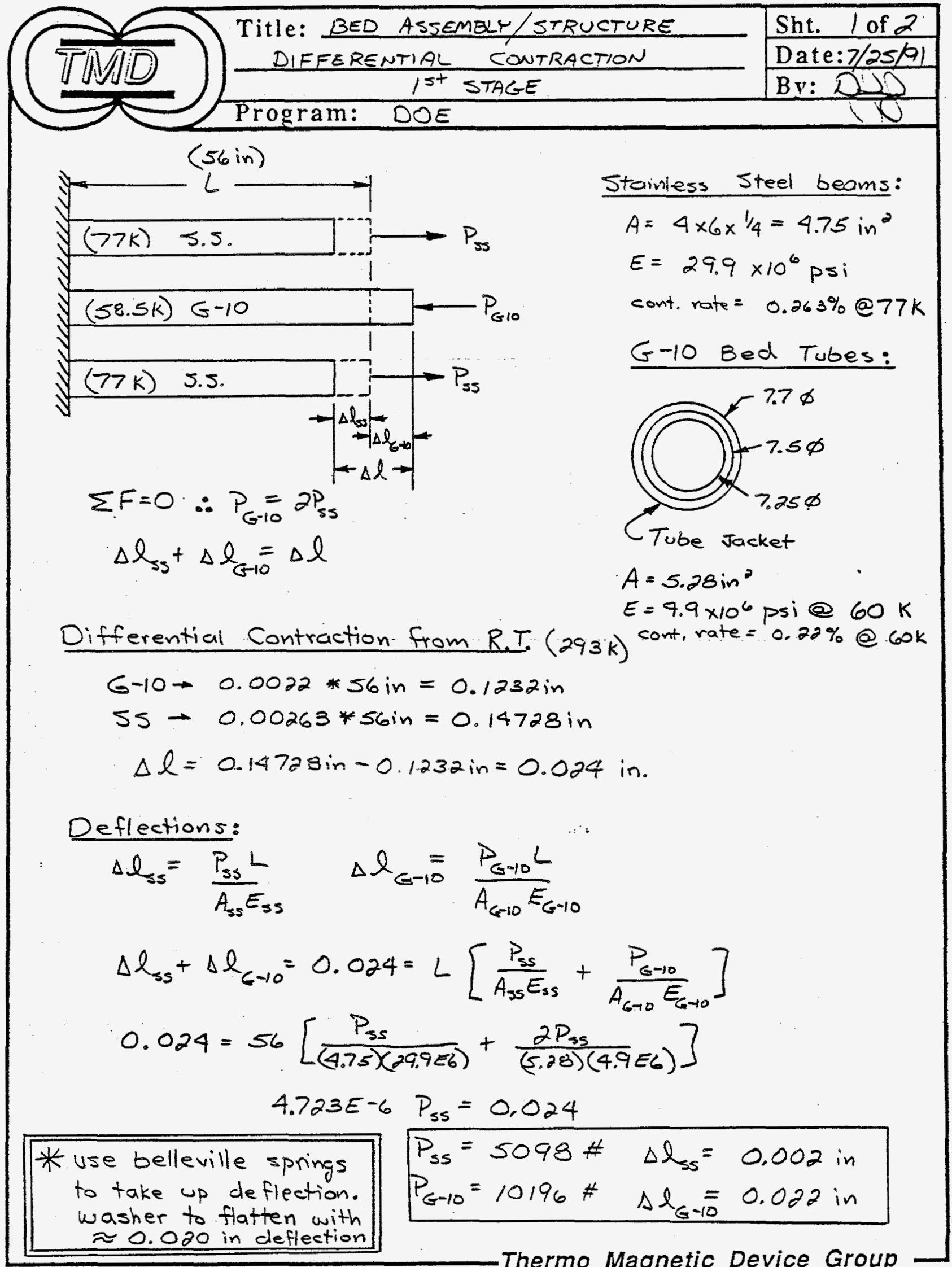

Thermo Magnetic Device Group 


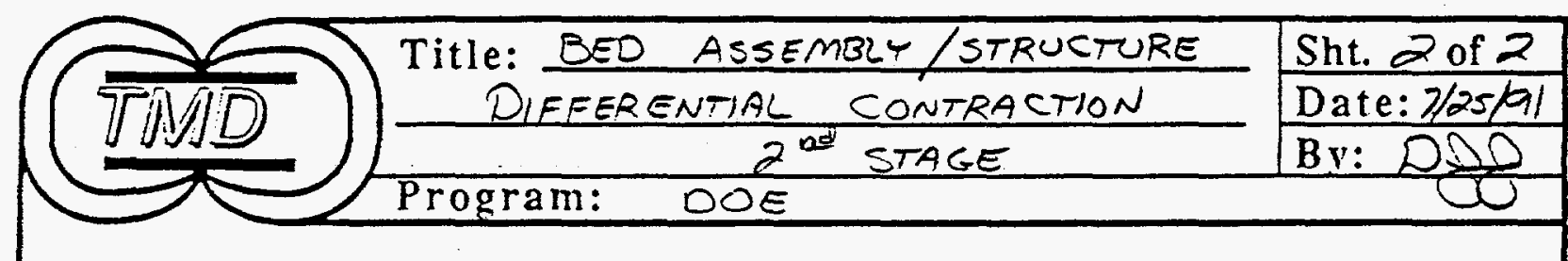

Differential Contraction from R.T. to $30 \mathrm{~K}$ :

SS $\rightarrow$ same as $77 k=0.14728 \mathrm{in}$

$\leftarrow-10 \rightarrow 0.0024 * 56=0.1344 \mathrm{in}$

$$
\Delta l=0.14728-0.1344=0.01288
$$

Deflections:

$$
\begin{aligned}
0.01288= & 56\left[\frac{P_{s s}}{(4.75)(29.9 E 6)}+\frac{2 P_{s s}}{(5.28)\left(4.9 \varepsilon_{6}\right)}\right] \\
4.723 E-6 P_{s s} & =0.01288 \\
P_{s S} & =2736 \# \quad \Delta l_{s s}=0.001 \\
P_{G-10} & =5472 \# \Delta l_{G-10}=0.012
\end{aligned}
$$

* use belleville springs to take up deflection. washer to flatten with $\approx 0.010 \mathrm{in}$ deflection.

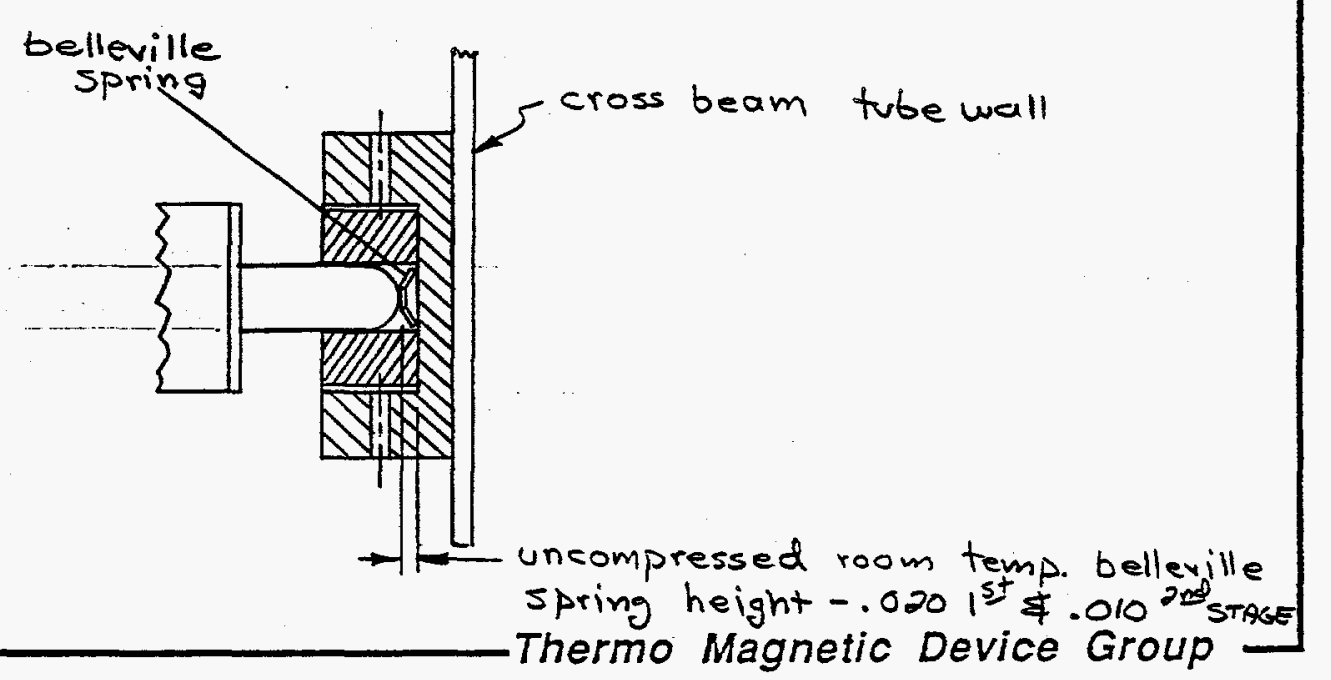

ASTRONAUTICS CORPORATION OF AMERICA. TECHNOLOGY CENTER

$$
\text { A-3 }
$$


Appendix B

Bed Assembly Internal

Parasitic Heat Leak Summary 


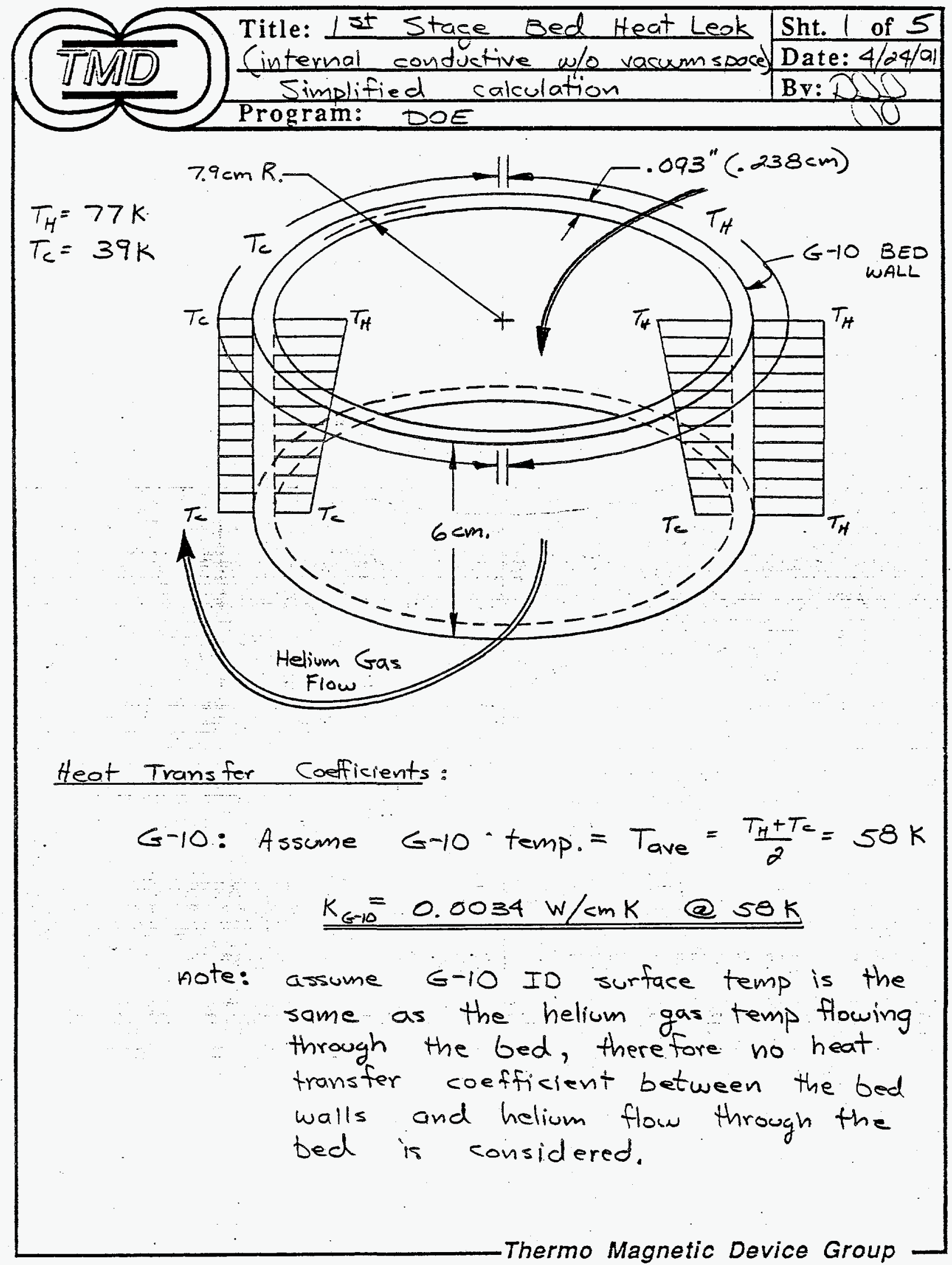


Title: Space Bed Heat Leak

Shit. 2 of 5

Date: $4 / 24 / a 1$

Program: DOE

Br: 000

Heat Transfer Coefficients (cont.)

6-10 to Helium: heat transfer between the bed (in annulus) $O D$ and the helium flow stream.

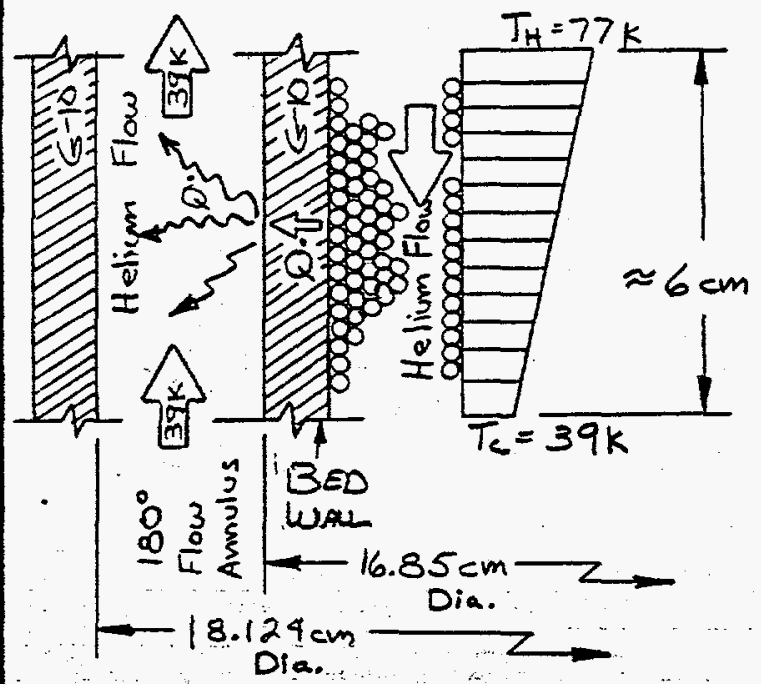

Use analogy of heat transfer between flowing gas and plates in a parallel plate regenerator.

$$
\begin{aligned}
& \text { Reynolds Number } N_{R}=\frac{\dot{m} d_{h}}{a A_{c} \mu} \\
& \dot{m}=\text { He mass flow rate }=435 \mathrm{~g} / \mathrm{s} \\
& d_{h}=\text { hydraulic dia }=\text { width of flow annulus }=.637 \mathrm{~cm} \\
& \alpha=\text { porosity }=1.0 \text {. } \\
& \alpha A_{c}=\text { cross section of flow area }=16.9 \mathrm{~cm}^{2} \\
& \mu=v i s c a s i t y\left(H_{e} @ 39 \mathrm{~K} \neq 20 \mathrm{~atm}\right)=58.810^{-6} \in / \mathrm{cms} \\
& N_{R}=\frac{(435 \mathrm{~g} / \mathrm{s})(.637 \mathrm{~cm})}{(1)\left(16.9 \mathrm{~cm}^{2}\right)\left(58.810^{-6} \mathrm{~g} / \mathrm{cms}\right)}=278,846 \\
& N_{\text {usselt number } @ \text { constant heat trans for rate } N_{N_{u}, H}} \\
& N_{N_{u, H}}=0.021 \mathrm{~N}_{R}^{0.8} \mathrm{~N}_{\mathrm{pr}} 0.6
\end{aligned}
$$

Thermo Magnetic Device Group -

ASTRONAUTICS CORPORATION OF AMERICA - TECHNOLOGY CENTER

B-3 


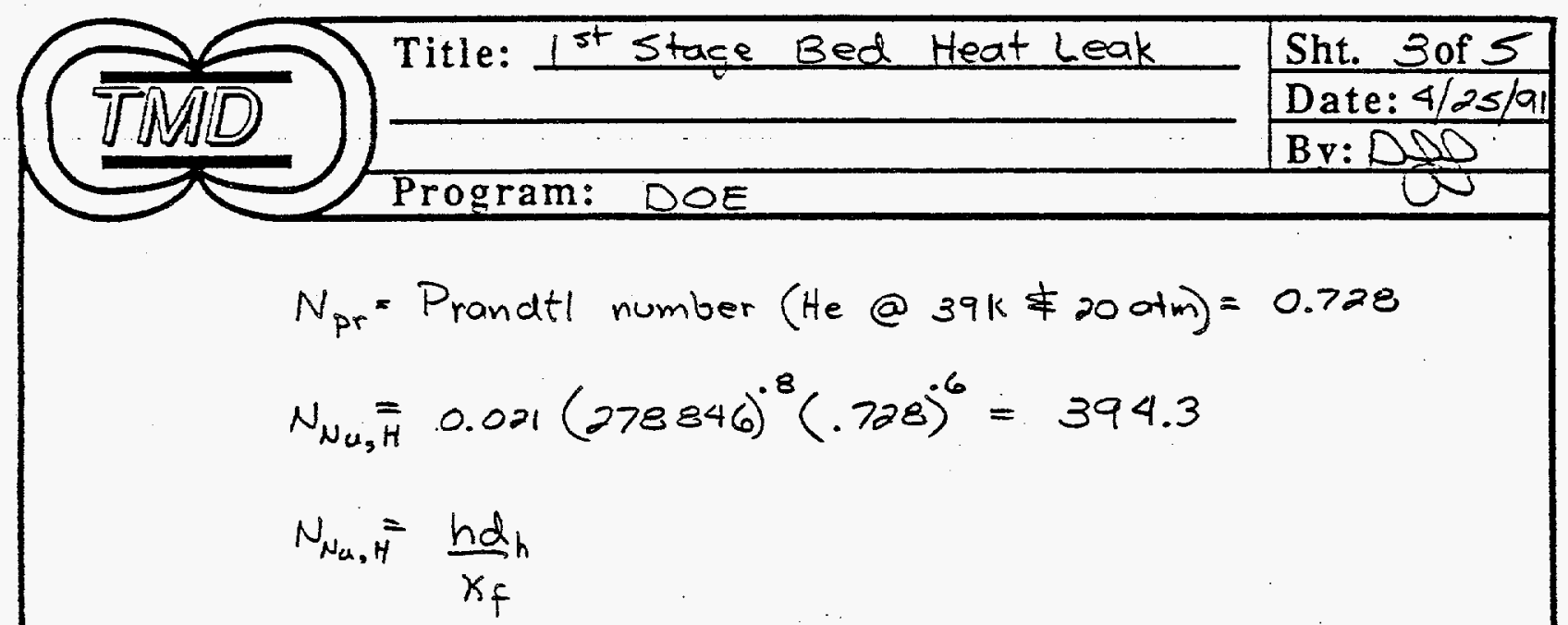

$x_{f}=$ thermal conductivity of fluid ( $H_{e} @ 39 k \$ 20 \mathrm{~atm}$ )

$$
\begin{gathered}
K_{f}=.000439 \mathrm{~W} / \mathrm{cmk} \\
h=\frac{N_{N a, H} K_{f}}{d_{h}}=\frac{(394.3)(.000439 \mathrm{~W} / \mathrm{cmk})}{.637 \mathrm{~cm}} \\
\frac{\therefore h=0.272 \mathrm{~W} / \mathrm{cm}^{2} \mathrm{~K}}{\text { heat transfer coefficient. }}
\end{gathered}
$$

Heat Leak Through Wall:

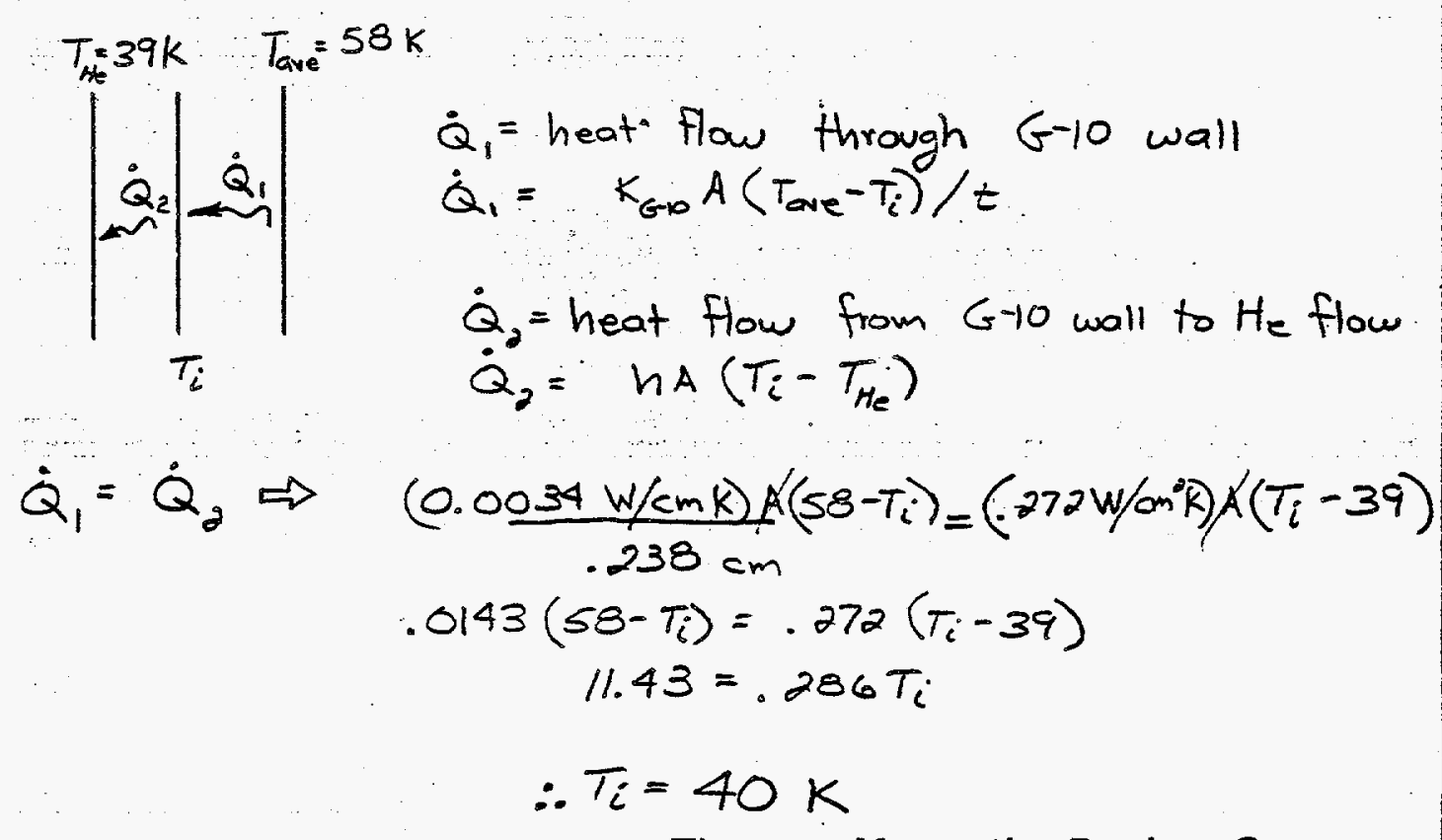

Therm Magnetic Device Group

ASTRONAUTICS CORPORATION OF AMERICA - TECHNOLOGY CENTER B-4 


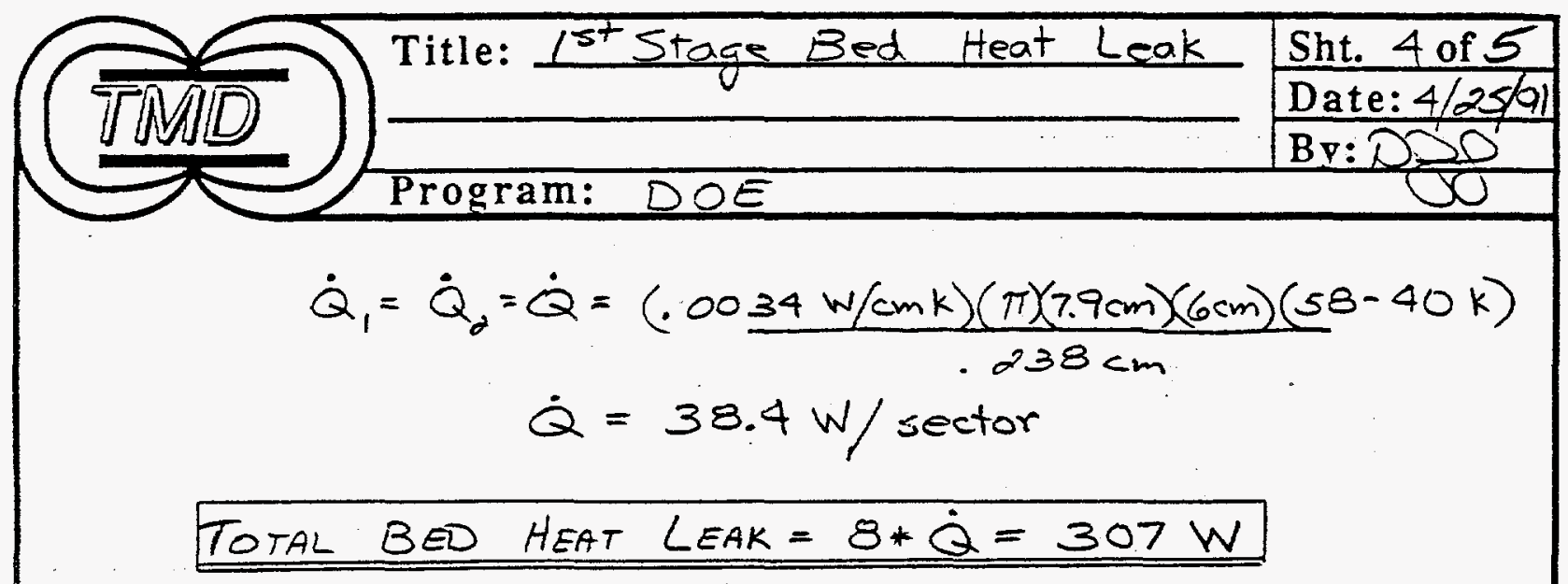

This is not an acceptable heat leak. Therefore add $a$ second wall with an evacuated space between the two walls.

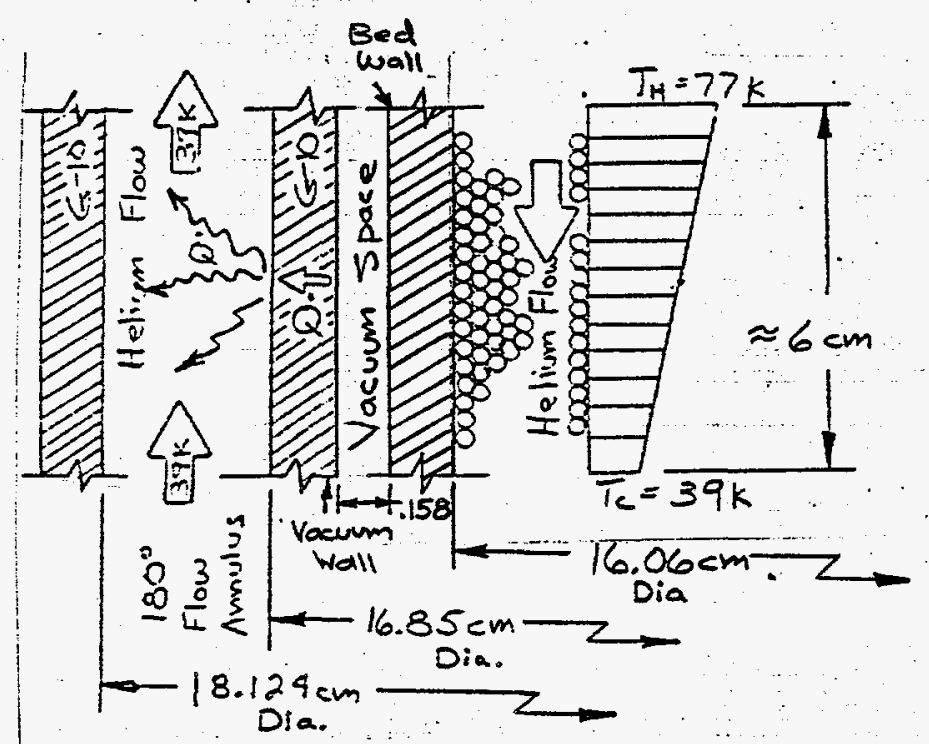

Assume a leak occurs and the evacuated space becomes filled with He @ $\approx 55 \mathrm{~K}$

$$
K_{H_{e}}=.000528 \mathrm{~W} / \mathrm{cmk}
$$

Overall heat transfer coefficient $\frac{1}{H}=\frac{1}{k_{G-10}}+\frac{1}{k_{H-}}+\frac{1}{k_{G-10}}+\frac{1}{n}$

$$
\begin{aligned}
& \frac{1}{H}=\frac{1}{\frac{.0034}{.238}}+\frac{1}{\frac{.00050}{.158}}+\frac{1}{\frac{.0034}{.238}}+\frac{1}{.272}=443 \\
& H=0.0023 \mathrm{~W} / \mathrm{cm}^{\circ} \mathrm{K} \\
& \dot{Q}=\left(.0023 \mathrm{~W} / \mathrm{cm}^{\circ} \mathrm{K}\right)(\pi)(8.23 \mathrm{~cm} \mathrm{R})(6 \mathrm{~cm})(58 \mathrm{k}-39 \mathrm{~K})
\end{aligned}
$$

Thermo Magnetic Device Group

ASTRONAUTICS CORPORATION OF AMERICA - TECHNOLOGY CENTER

B-5 


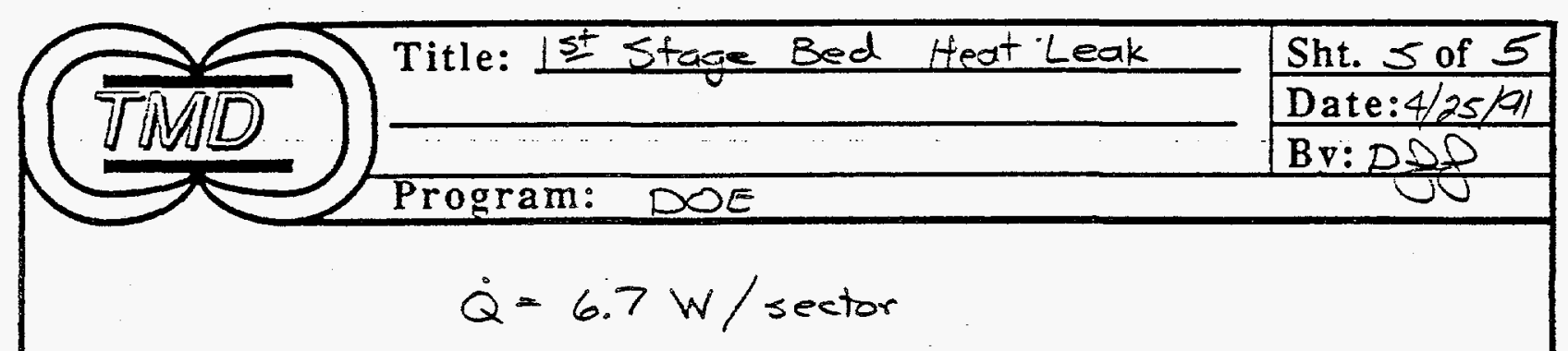

Circumferential Heat Leak in Vacuum wall:

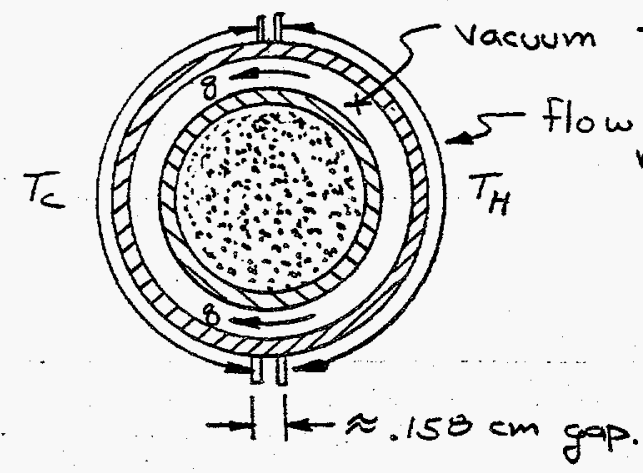

$$
\begin{aligned}
& q=\frac{(0.0034 \mathrm{w} / \mathrm{kmk})(6 \mathrm{~cm})(.238 \mathrm{~cm})(77 k-39 k)}{.158 \mathrm{~cm}} \\
& q=1.2 \mathrm{~W} / \mathrm{side}
\end{aligned}
$$

Total Bed Heat Leak:

$$
\begin{aligned}
& \dot{Q}=8 *(6.7 \mathrm{~W} / \text { sector }+1.2 \mathrm{~W} / \text { side }) \\
& \dot{Q}=63 \mathrm{~W} \text { TOTAL BED HEAT LEAK }
\end{aligned}
$$

$w /$ helium leaked into evacuated space

$$
\dot{Q}=54 \mathrm{~W} \text { TOTAL BES HEAT LEAK }
$$

wo helium leaked into evacuated space

Thermo Magnetic Device Group

ASTRONAUTICS CORPORATION OF AMERICA - TECHNOLOGY CENTER 
Appendix C

Appendix C - Symbols and Usage

$\mathrm{C}-1$ 


\section{Base Units}

\begin{tabular}{|lll|}
\hline Quantity & Unit & SI Symbol \\
\hline & & \\
length & meter & $\mathrm{m}$ \\
mass & kilogram & $\mathrm{kg}$ \\
time & second & $\mathrm{s}$ \\
electric current & ampere & $\mathrm{A}$ \\
thermodynamic temperature & kelvin & $\mathrm{K}$ \\
amount of substance & mole & $\mathrm{mol}$ \\
luminous intensity & candela & $\mathrm{cd}$ \\
\hline
\end{tabular}

\begin{tabular}{|lll|}
\hline Supplementary Units & & \\
\hline & & \\
plane angle & radian & $\mathrm{rad}$ \\
solid angle & steradian & $\mathrm{sr}$ \\
\hline
\end{tabular}




\begin{tabular}{|c|c|c|c|}
\hline Quantity & Unit & Symbol & SI Formula \\
\hline \multicolumn{4}{|l|}{ Derived Units } \\
\hline absorbed dose & gray & Gy & $\mathrm{J} / \mathrm{kg}$ \\
\hline acceleration & meter per second squared & -- & $\mathrm{m} / \mathrm{s}^{2}$ \\
\hline activity (radioactive) & bequerel & $\mathrm{Bq}$ & $1 / s$ \\
\hline activity (radioactive) & disintegration per second & $\ldots$ & $(\mathrm{dis}) / \mathrm{s}$ \\
\hline angular acceleration & radian per second squared & --- & $\mathrm{rad} / \mathrm{s}^{2}$ \\
\hline angular velocity & radian per second & --- & $\mathrm{rad} / \mathrm{s}$ \\
\hline area & square meter & --- & $\mathrm{m}^{2}$ \\
\hline density & kilogram per cubic meter & -- & $\mathrm{kg} / \mathrm{m}^{3}$ \\
\hline electric capacitance & farad & $\mathrm{F}$ & $A \cdot s / V$ \\
\hline electrical conductance & siemens & $S$ & $\mathrm{~A} / \mathrm{V}$ \\
\hline electric field strength & volt per meter & --- & $\mathrm{V} / \mathrm{m}$ \\
\hline electric inductance & henry & $\mathrm{H}$ & $\mathrm{V} \cdot \mathrm{s} / \mathrm{A}$ \\
\hline electric potential difference & volt & V & W/A \\
\hline electric resistance & ohm & $\Omega$ & $\mathrm{V} / \mathrm{A}$ \\
\hline electromotive force & volt & $\mathrm{V}$ & W/A \\
\hline energy & joule & $J$ & $N \cdot m$ \\
\hline entropy & joule per kelvin & -- & $\mathrm{J} / \mathrm{K}$ \\
\hline force & newton & $\mathbf{N}$ & $\mathrm{kg} \cdot \mathrm{m} / \mathrm{s}^{2}$ \\
\hline frequency & hertz & $\mathrm{Hz}$ & $1 / \mathrm{s}$ \\
\hline illuminance & $\operatorname{lux}$ & $1 \mathrm{x}$ & $\mathrm{lm} / \mathrm{m}^{2}$ \\
\hline luminance & candela per square meter & -- & $\mathrm{cd} / \mathrm{m}^{2}$ \\
\hline Iuminous flux & lumen & $\operatorname{lm}$ & $\mathrm{cd} \cdot \mathrm{sr}$ \\
\hline magnetic field strength & ampere per meter & -- & $\mathrm{A} / \mathrm{m}$ \\
\hline magnetic flux & weber & $\mathrm{Wb}$ & $\mathrm{V} \cdot \mathrm{s}$ \\
\hline magnetic flux density & tesla & $\mathrm{T}$ & $\mathrm{Wb} / \mathrm{m}^{2}$ \\
\hline magnetomotive force & ampere & A & $\cdots$ \\
\hline power & watt & W & $\mathrm{J} / \mathrm{s}$ \\
\hline pressure & pascal & $\mathrm{Pa}$ & $\mathrm{N} / \mathrm{m}^{2}$ \\
\hline quantity of electricity & coulomb & C & $A \cdot S$ \\
\hline quantity of heat & joule & $\mathbf{J}$ & $\mathrm{N} \cdot \mathrm{m}$ \\
\hline radiant intensity & watt per steradian & -- & $\mathrm{W} / \mathrm{sr}$ \\
\hline specific heat & joule per kilogram-kelvin & --- & $\mathrm{J} / \mathrm{kg} \bullet \mathrm{K}$ \\
\hline stress & pascal & $\mathrm{Pa}$ & $\mathrm{N} / \mathrm{m}^{2}$ \\
\hline thermal conductivity & watt per meter-kelvin & -- & $\mathrm{W} / \mathrm{m} \cdot \mathrm{K}$ \\
\hline velocity & meter per second & -- & $\mathrm{m} / \mathrm{s}$ \\
\hline viscosity, dynamic & pascal-second & --- & $\mathrm{Pa} \cdot \mathrm{s}$ \\
\hline viscosity, kinematic & square meter per second & -- & $\mathrm{m}^{2} / \mathrm{s}$ \\
\hline voltage & volt & $\mathrm{V}$ & W/A \\
\hline volume & cubic meter & -- & $\mathrm{m}^{3}$ \\
\hline wave number & reciprocal meter & --- & (wave) $/ \mathrm{m}$ \\
\hline work & joule & $\mathbf{J}$ & $\mathrm{N} \cdot \mathrm{m}$ \\
\hline
\end{tabular}

